\title{
THE SKEWNESS IN EXPECTED MACRO FUNDAMENTALS AND PREDICTABILITY OF EQUITY RETURNS: EVIDENCE AND THEORY
}

\begin{abstract}
Wasin Siwasarit
A dissertation submitted to the faculty of the University of North Carolina at Chapel Hill in partial fulfillment of the requirements for the degree of Doctor of Philosophy in the Department of Economics.
\end{abstract}

Chapel Hill

2015

Approved by:

Eric Ghysels

Riccardo Colacito

Anusha Chari

Mariano M. Croce

Toan Phan 
(C) 2015

Wasin Siwasarit

ALL RIGHTS RESERVED 


\section{ABSTRACT \\ WASIN SIWASARIT: THE SKEWNESS IN EXPECTED MACRO FUNDAMENTALS AND PREDICTABILITY OF EQUITY RETURNS: EVIDENCE AND THEORY. (Under the direction of Eric Ghysels and Riccardo Colacito.)}

We document that the first and third cross-sectional moments of the distribution of GDP growth rates made by professional forecasters can predict equity excess returns, a finding which is robust to controlling for a large set of well established predictive factors. We show that introducing time-varying skewness in the distribution of expected growth prospects in an otherwise standard endowment economy can substantially increase the model implied equity Sharpe ratios, and produce a large amount of fluctuation in equity risk premia. 


\section{ACKNOWLEDGMENTS}

There are more than 150,681, 600 seconds (4 years, 9 months, 9days) that I have stayed in Chapel Hill, North Carolina. Undoubtedly, all the scenes, moments, are still vivid and heartfelt every time when I remind to it. I therefore would like to express a deep appreciation to these individuals for their supports and contributions, in one way or the other, to this dissertation.

First and foremost, I am particularly indebted to my remarkably intellectual advisors, Prof. Eric Ghysels and Prof. Riccardo Colacito. I have met them in my second-year classes which are Introduction to Empirical Finance and Foundations of Macro-Finance. In summer of that year, I had a great opportunity to work with them for my field paper in financial econometrics. I have learned a lot from working closely with them. After passing the field paper examination, I continued working with them as their advisee. We regularly had the meeting every week to update my research progress. In our meeting environment, we always enjoy solving the problems we found in our research and we always happy with the results we had. During three years that I worked with them, more than 150 meetings in Ric's office and on Skype calls, I can definitely say that I enjoyed every minute of it!. Thanks so much for being the strong and supportive advisors to me.

I am also very grateful to Prof. Anusha Chari, Prof. Mariano M. Croce, and Prof. Toan Phan, who made innumerable and invaluable comments and suggestions. Taking Prof. Chari 's class in International Finance and Prof. Croce's class in Methods in Macro-Finance inspires me in doing the Macro-Finance research. I always recommend the second-year students to take these two classes.

I must also acknowledge the great support given by professor and director of graduate studies: Prof. Helen V. Tauchen, the staff of the department of economics: Kenlyn Merritt and Cindy Wunder. I also thank Joy Patterson and Marion Strandh for their excellent supports at Kenan-Flagler Business school. 
I thank Prof. Pab Jotikasthira for giving me a good opportunity to work as his TA and RA at Kenan-Flagler Business School.

Living in a small city like Chapel hill is boring if I don't have such groups of friends to do activities together. I am very thankful my writing group at UNC writing center: Dr. Gigi Taylor, Percival Guevarra, Vicky Yeh, Soo Jin Lee, and Geovani Ramirez. I have been especially fortunate to have a chance to know and join the meditation group at UNC: Linda Chupkowski, Christina Lebonville, Lauren Townsend,and Alden Adrion-Thanks so much for your support, friendship, and hospitality.

I want to thank my good friends at department of economics, department of statistics, and department of finance: Ben, Brian, Melati, Yiyi, Hanwei, Pragya, Derya, Daniel, Laura, Atet, Roberto, Tao, Jinghan, Sunjin.

I owe a special debt to $\mathrm{P}^{\prime} \mathrm{Pi}, \mathrm{P}^{\prime}$ Nuch, $\mathrm{P}^{\prime}$ Kub, Nase, $\mathrm{P}^{\prime}$ Rong, $\mathrm{N}^{\prime}$ Paula, and $\mathrm{N}^{\prime}$ Wai for being my true friends. Especially, $\mathrm{P}^{\prime} \mathrm{Pi}$, she helped me to accept the sudden loss of my father and travelled back to U.S with me after I finished with my father's funeral in Thailand. I also thank my Thais friends that I have made in Chapel hill for their support and friendship.

I thank Linda Longs and her family. It is my privilege to stay with you while I studied here. Your love, care, and support are richly appreciated. I will definitely miss you when I go back to Thailand and will be looking forward to seeing you in Bangkok or when I come back to U.S.

I thank William Young and Madeline Young for being my best host family since my first-year in Chapel hill.

Along with the above people, I wish to express my special thanks to Thammasat University for supporting me with generous fellowships when I studied at University of North Carolina at Chapel Hill.

Finally, I want to offer a special thanks to my family for their love, support, sacrifice, kind indulgence, and understanding. I also would like to express my sincerest gratitude to my grandmother, my grandfather ,and my father. Although they passed away, their love, support, and care is still in my heart. I own this to them.

All these acknowledgment notwithstanding, responsibility for the dissertation is mine. If any of readers has any comments or suggestions, please bring them to my attention. Any comments will be deeply appreciated. 
To my mom and dad who always support my dream

To everyone who has a dream and believe in it! 


\section{TABLE OF CONTENTS}

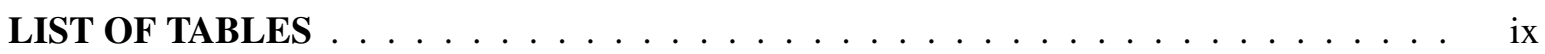

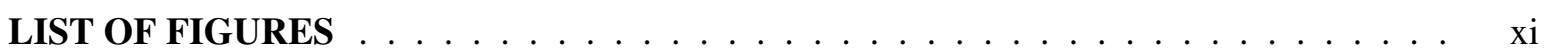

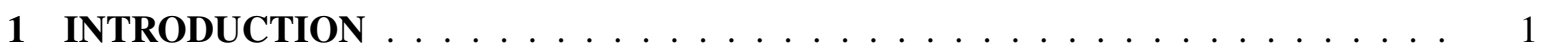

2 TIME SERIES PROPERTIES OF THE CROSS-SECTIONAL MOMENTS OF THE DISTRIBUTION OF EXPECTED REAL GDP GROWTH RATES . . . . . 5

2.1 Time series properties of the cross-section of expected GDP growth $\ldots \ldots$

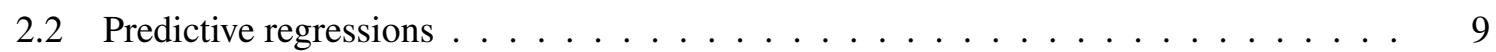

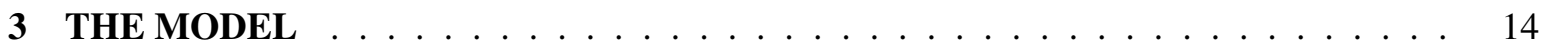

3.1 A model with time-varying mean and skewness . . . . . . . . . . . . . . . . . 14

3.2 A model with time-varying volatility and skewness $\ldots \ldots \ldots \ldots$

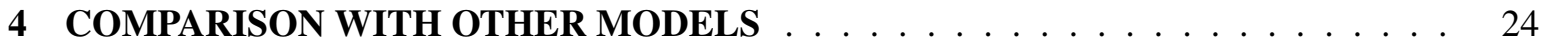

4.1 Skewness in consumption based asset pricing models . . . . . . . . . . . . . 24

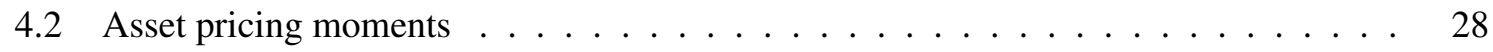

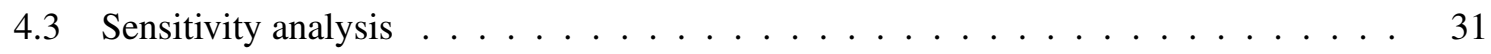

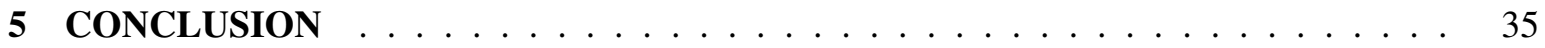

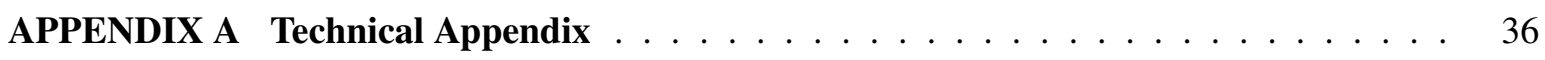

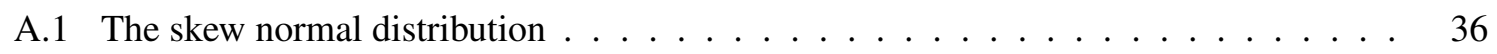

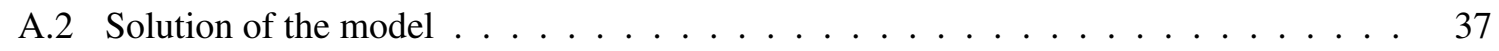

A.3 Solution for the constant volatility case . . . . . . . . . . . . . . . . 39

A.4 Solution of the model with time-varying volatility and skewness . . . . . . . . . 43

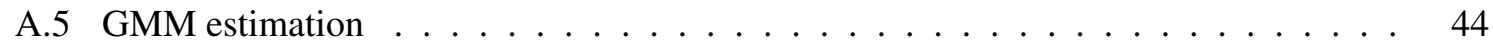

A.6 Conditional moments in the model with jumps . . . . . . . . . . . . . . . 47 
A.7 Time series properties for alternative data configurations $\ldots \ldots \ldots \ldots$

A.8 Robustness of predictive regressions . . . . . . . . . . . . . . . . . . . . . 49

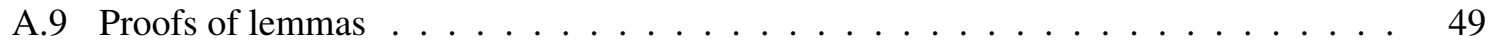

A.10 Approximations . . . . . . . . . . . . . . . . . . 77

A.11 Analytical solution of the case with time-varying volatility and skewness . . . . . . 78

A.12 Additional calculations for GMM estimation . . . . . . . . . . . . . . . . . . . 89

A.13 Numerical algorithm . . . . . . . . . . . . . . . . . . . 94

A.14 Comparison of approximations . . . . . . . . . . . . . . . . . 97

A.15 Models with jumps $\ldots \ldots \ldots \ldots \ldots \ldots \ldots$

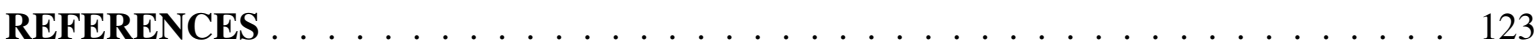




\section{LIST OF TABLES}

2.1 Time Series Properties of Cross-Sectional Moments . . . . . . . . . . . . . . . . . 8

2.2 Predictive Regressions $\ldots \ldots \ldots \ldots \ldots \ldots$

2.3 Correlation between Predictors . . . . . . . . . . . . . . . . . . . . . . . 12

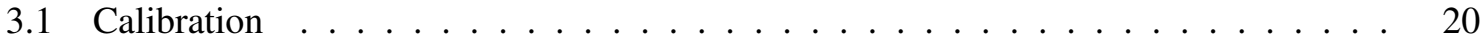

3.2 Model Implied Predictive Regressions at Semi-annual Frequency . . . . . . . . . . . 22

3.3 Correlation of Excess Returns: Quintile Analysis _ . . . . . . . . . . . . . . . 23

4.1 Monthly Calibration . . . . . . . . . . . . . . . . . . . . . . 25

4.2 Comparison of Models . . . . . . . . . . . . . . . . . . . . . . . . . . . . 29

4.3 The Role of Skewness in Predictive Regressions . . . . . . . . . . . . . . . . . . 30

4.4 Sensitivity Analysis . . . . . . . . . . . . . . . . . . . . . 33

A.1 Time Series Properties of Cross-Sectional Moments . . . . . . . . . . . . . . . . 50

A.2 Predictive Regressions: Livingston + Blue Chip (ALL) _ . . . . . . . . . . 52

A.3 Predictive Regressions:Livingston (cross-sectional size $>$ 20) + Blue Chip . . . . . . 54

A.4 Predictive Regressions: Livingston (cross-sectional size > 30) + Blue Chip _ . . . 56

A.5 Predictive Regressions: Livingston + Blue Chip (ALL)

with Bootstrap Standard Deviations _ . . . . . . . . . . . . . . . 58

A.6 Predictive Regressions: Livingston (up to 98) + Blue Chip with Bootstrap Standard Deviations _. . . . . . . . . . . . . . . . . 60

A.7 Predictive Regressions:Livingston (cross-sectional size > 20) + Blue Chip with Bootstrap Standard Deviations . . . . . . . . . . . . . . . . 62

A.8 Predictive Regressions: Livingston (cross-sectional size > 30) + Blue Chip with Bootstrap Standard Deviations _. . . . . . . . . . . . . . . 64

A.9 Predictive Regressions: Livingston + Blue chip (ALL) with Kurtosis . . . . . . . . . 66

A.10 Predictive Regressions: Livingston (up to 98) + Blue Chip with Kurtosis . . . . . . . 68 
A.11 Predictive Regressions:Livingston (cross-sectional size $>20)+$ Blue Chip with Kurtosis . . . . . . . . . . . . . . . . . . . . . 70

A.12 Predictive Regressions: Livingston (cross-sectional size $>30)+$ Blue Chip with Kurtosis . . . . . . . . . . . . . . . . . . . . . . . . 72

A.13 Predictive Regressions (Delete Overlap from 1990S1-1997S2) . . . . . . . . . . 74

A.14 Maximum Percentage Error of Volatility Approximation . . . . . . . . . . 77

A.15 Summary Statistics of Consumption Growth Rate . . . . . . . . . . . . . . . . 93

A.16 Comparison of Analytical Solutions for the Constant Volatility Case . . . . . . . . . 107

A.17 Comparison of Analytical and Numerical Solutions of Utility Function for Constant Volatility Case . . . . . . . . . . . . . . . . . . . . . . . . 109

A.18 Comparison of Analytical and Numerical Solutions of Log Price-Dividend Ratio for Constant Volatility Case . . . . . . . . . . . . . . . . . . . . . . . . 119

A.19 Comparison of Analytical and Numerical Solutions of Utility Function in the Model with Time-Varying Volatility and Skewness . . . . . . . . . . . 120

A.20 Comparison of Analytical and Numerical Solutions of Log Price-Dividend Ratio in the Model with Time-Varying Volatility and Skewness . . . . . . . . . . . 121

A.21 Sensitivity Analysis: Model with Jumps _ . . . . . . . . . . . . . . . . . . . 122 


\section{LIST OF FIGURES}

2.1 Time Series for The First Three Cross-Sectional Moments of The Distribution of Expected Real GDP Growth. . . . . . . . . . . . . . . . 6

3.1 Utility Function. . . . . . . . . . . . . . . . . . . . . . . 21

4.1 Comparison of Conditional Skewness of Expected Consumption Growth Across Models. . . . . . . . . . . . . . . . . . . . . 27 


\section{CHAPTER 1: INTRODUCTION}

Each month a large number of forecasts about expected growth prospects of the economy is made available to the public. A lot of attention is typically devoted to the average or median of these forecasts, sometimes called the consensus forecast. We document that the cross-sectional moments of

the distribution of professional forecasters' expected GDP growth help predict future equity excess returns. In particular, we show that the first and third cross-sectional moments have economically and statistically significant predictive power, as larger mean and more positive skewness predict lower equity returns going forward. This empirical result remains even after one controls for standard predictors such as cay (as in Lettau and Ludvigson (2001)), dividend yields, default premia, VIX, variance risk premium, realized variance of the S\&P 500 (Bollerslev et al. (2009)) or the so called Fear index (Bollerslev and Todorov (2011)).

The empirical evidence of return predictability for asymmetry poses a serious challenge as standard asset pricing models, which are inherently symmetric, do not feature such patterns. We address this challenge by building a model which mimics the patterns we discover with the aforementioned predictive regressions. We follow Bansal and Yaron (2004) by assuming that investors order consumption profiles using Epstein and Zin (1989) preferences. This means that agents care about the temporal distribution of risk. In particular, we show that this type of investors not only likes high expected utility levels and positive asymmetry about future outcomes, but also dislikes uncertainty and negative asymmetry about future wealth.

We explicitly model the expected growth rate of consumption as following a skew-normal distribution with time-varying parameters. First introduced by Azzalini (1985), the skew-normal provides a convenient way of modeling asymmetric distributions, as the first three conditional moments are available in closed form. We discipline the dynamics of the conditional moments, by using the observed distribution of the cross-section of analysts' forecasts. Furthermore, we can easily incorporate the empirical finding that the skewness of expected macro forecasts appears to have predictive power 
for future revisions of the conditional mean. In addition to the numerical solution of the model, we provide approximate analytical solutions and show that the latter provide an accurate description of the model's solution. We also characterize the pricing kernel as a function of the shocks in the model and derive conditional equity risk premia. This allows us to document the predictive power of skewness as determined by our model.

We perform a direct validation of the model, by feeding the actual time-series of mean, volatility, and skewness of analysts' forecasts in the model's solution to document that conditional expectations of future returns are quite correlated with future realized returns. Furthermore, we show that the introduction of skewness can: (i) substantially increase equity risk premia, and (ii) produce a large amount of time variation in conditional risk premia. What is also interesting to note is that the theoretical model we develop not only features time-varying skewness, but attributes a special role to an interaction term of skewness and standard deviation - acting like a signed scaled variance term. The empirical support for this term is even more striking than the actual skewness itself.

We compare the performance of our model relative to the recent strand of the literature that has looked at jumps as a way of introducing tail risk. For example, Bansal and Shaliastovich (2013) present an equilibrium model in which fluctuations in investors confidence about expected growth lead to variation in risk premia. Their economy set-up follows the long-run risks specification of Bansal and Yaron (2004), and features a Gaussian consumption growth process with time-varying expected growth and volatility. In a related paper, Drechsler and Yaron (2011) consider the possibility of jumps in both the level and the volatility of the expected growth rate of consumption. A common feature of these recent models is that the dynamics of the skewness of expected macroeconomic fundamentals is tied to the dynamics of volatility. Our analysis, using a skew-normal distribution with time-varying parameters, allows us to introduce an additional skewness factor, which turns out to be quite important. For example, in the Drechsler and Yaron (2011) model, periods of high volatility are associated to periods of low skewness in absolute value, while times of low macroeconomic uncertainty coincide with an increased amount of left tail risk. The tied link between volatility and skewness makes it difficult for the existing models to match the signs of relevant coefficients in predictive regressions. This feature of the existing models represents a strong argument for including a separate factor to model the dynamics of skewness. Our model does this in a parsimonious fashion and enables us 
to match the signs and magnitudes of relevant parameters in predictive regressions, while keeping the skewness factor empirically plausible and tied to the aforementioned cross-sectional moments of expected growth.

This dissertation is related to several strands of the literature. On the empirical side, an extensive literature has documented the predictability of equity excess returns at various horizons (see inter alia Welch and Goyal (2008)). Campbell and Diebold (2009) have provided evidence in support of the predictive power of the consensus forecast for subsequent stock market returns. It is noteworthy that, while the coefficient on average expected growth is always strongly statistically significant, the one on the dispersion is typically not. We extend their findings and show that the degree of asymmetry helps explaining equity returns going forward. Remarkably, asymmetry matters far more than dispersion does, i.e. the third moment matters while the second does not. Furthermore, there is a considerable literature on asset pricing models with investors who take into account higher moments (beyond variances) in asset returns. Arditti (1967), Rubinstein (1973), Kraus and Litzenberger (1976), and Harvey and Siddique (2000) developed some of the early models of expected returns which incorporate the higher moments of individual securities that co-move with the aggregate market portfolio. Similarly, Christoffersen and Diebold (2006) emphasize the predictability in asset return signs, and show how it remains valid with time-varying conditional skewness and/or kurtosis.

On the modeling side, our work is related to the recent literature that has looked at the introduction of non-Gaussian innovations in the dynamics of consumption. Yang (2011) notes that the empirical distribution of durable consumption growth is left-skewed. He shows that a parsimonious model that includes this empirical feature of durable consumption significantly improves the performance of the long-run risks model, by allowing it to produce counter-cyclical bond risk premiums, and an upward sloping real term structure. Segal et al. (2015) use an extended version of the Bansal and Yaron (2004) model in which the shocks to consumption are divided into two separate zero-mean components which capture positive and negative growth innovations. Each component is affected by a time-varying volatility process, which determine a separate role for bad and good economic uncertainty. Bekaert and Engstrom (2010) have proposed a "Bad Environment-Good Environment" framework, in which they assume that consumption growth is exposed to two types of shocks, one 
with positive, and one with negative skewness, whose relative importance varies through time. ${ }^{1}$ They show that this richer consumption dynamics allows the habits' model by Campbell and Cochrane (1999) to account for the variance risk premium. We differentiate from these papers by proposing a model in which the dynamics of skewness are anchored to the observed degree of asymmetry of expected macroeconomic forecasts, and that speaks directly to our novel empirical evidence.

Our work emphasizes skewness in fundamentals, but we do not focus necessarily on rare disaster risks (see for example Wachter (2013)). Rather, we highlight the importance of skewness even during "normal" times. Moreover, our approach does not rely exclusively on negative skewness. Time varying skewness may be both positive and negative: both will matter in our approach. The work by Tsai and Wachter (2014) constitutes a notable exception, as they consider both the possibility of rare disasters and rare booms. Our work differs from theirs in that our skewness process is smoother due to the absence of jumps. Also related is Benzoni et al. (2011) who combine recursive preferences with a very large and rare shock to a persistent component in cash-flow growth rates to generate a steep implied-volatility smirk. In our work the movements in skewness are not simply driven by rare events and provide directional information about either up- or downside risks. Finally, our work is related to Chabi-Yo (2012) and Bakshi and Chabi-Yo (2014) that have pointed out the relevance of higher order moments for the construction of tighter bounds on the variance of pricing kernels.

The dissertation is organized as follows. The next chapter presents our main empirical findings by discussing the time-series properties of the cross-sectional moments of the distribution of expected GDP growth rates, as well as documenting the predictive power of skewness for future stock market returns. Chapter 3 presents a version of our model that can be solved in closed form and that is calibrated to match the time-series properties of the cross-sectional moments of the distribution of expected GDP growth rates. We use this model to explain how skewness affects equity returns and to document the crucial interaction between skewness and volatilities. Chapter 4 performs a comparison between our model and the model with jumps proposed by Drechsler and Yaron (2011). Chapter 5 concludes the work.

\footnotetext{
${ }^{1}$ In a related paper, Bekaert et al. (2015) develop the econometric framework to estimate the "Bad Environment-Good Environment" class of models.
} 


\section{CHAPTER 2: TIME SERIES PROPERTIES OF THE CROSS-SECTIONAL MOMENTS OF THE DISTRIBUTION OF EXPECTED REAL GDP GROWTH RATES}

Multiple forecasts are commonly available for key economic variables, as different professional analysts may disagree about the outlook of the economy, or, simply, because different forecasting models and/or information are employed in this task. In this chapter, we put ourselves in the position of an investor who looks at the entire cross-sectional distribution of these forecasts at each point in time. We document that this distribution features time-varying mean, volatility, and skewness. Campbell and Diebold (2009) find that the professional forecasters' cross-sectional distribution timevarying mean and volatility help predict future stock returns. Their empirical finding can be reconciled with a number of asset pricing models.

What we document next is both empirically novel and theoretically intriguing. We find that the cross-sectional skewness of analyst forecasts predicts future stock returns. The predictability of returns using cross-sectional skewness is even stronger than for the first two conditional moments and is robust to the inclusion of both standard predictors as well as commonly used measures of downside and/or distress risks. It is important to note that the cross-sectional skewness is not simply a measure of downside risk - as it also reflects upside growth states.

This chapter is organized as follows: in a first section we document the time series properties of the cross-section of expected GDP growth, while a second section covers predictive regressions. 


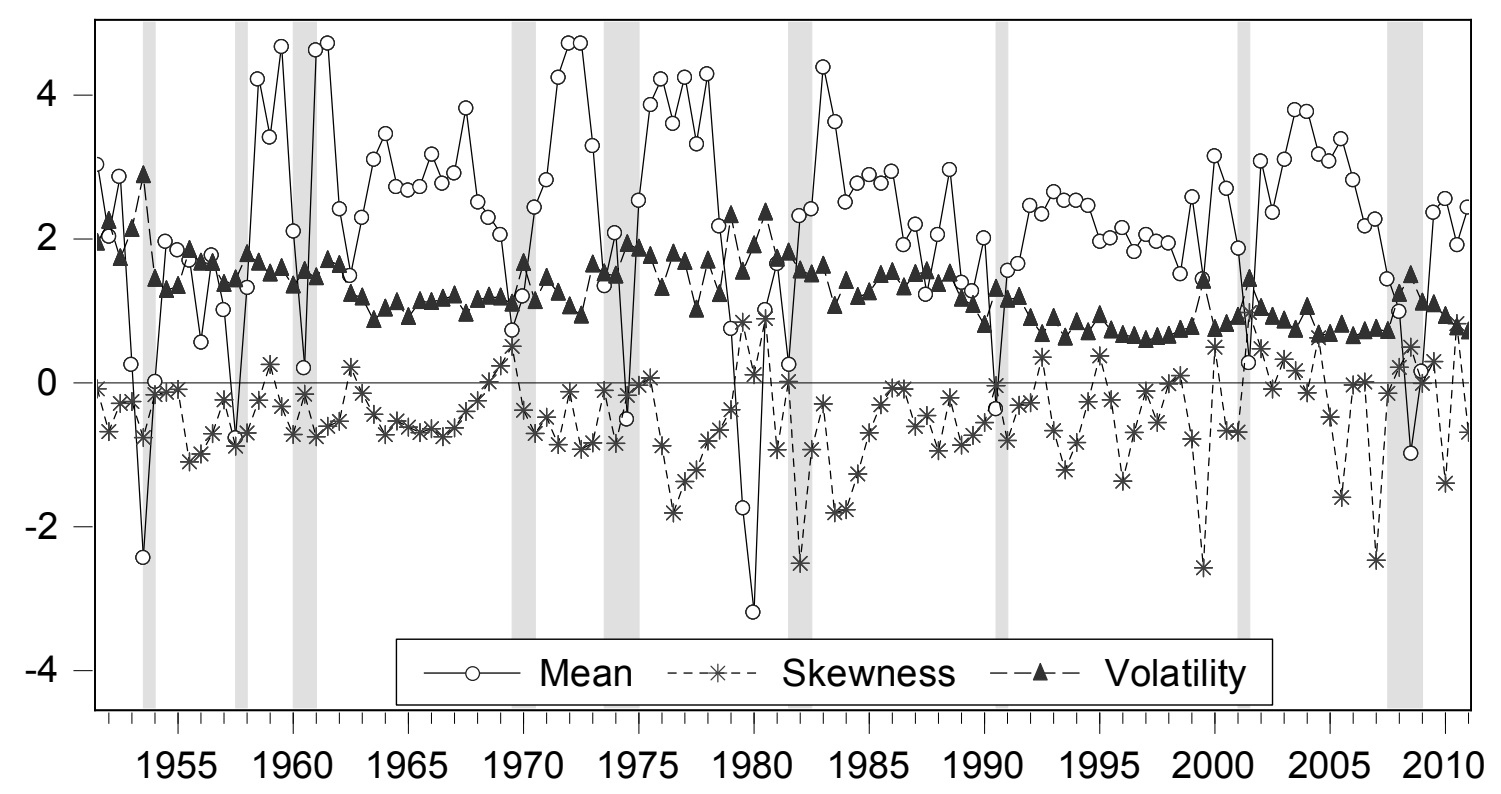

Figure 2.1: Time series for the first three cross-sectional moments of the distribution of expected real GDP growth. Mean stands for the median forecast, Variance stands for the interquartile measure of volatility (i.e. the 75 th percentile less the 25 th percentile), and Skewness is the quantile-based measure of skewness (i.e. the 75th percentile plus the 25th percentile minus twice the median). The series are constructed using one semester ahead real GDP growth forecasts from the Livingston and Blue Chip datasets from 1951:1 to 2011:2. The vertical grey bars represent recessions according to the National Bureau of Economic Research.

\subsection{Time series properties of the cross-section of expected GDP growth}

Dataset. We construct the time series of cross-sectional measures of mean, dispersion, and asymmetry of real GDP growth expectations by using two datasets. ${ }^{1}$ The first one is the Livingston Survey, which starts in 1946 and is the oldest continuous survey of economists' real GDP growth expectations. It summarizes the forecasts of economists from industry, government, banking, and academia. The Federal Reserve Bank of Philadelphia took responsibility for the survey in 1990. Every June and December, participants forecast a set of key macroeconomic variables, including real and nominal GDP. Survey participants are asked to provide forecasts for three horizons: (1) the end of the current month, (2) six months ahead, and (3) 12 months ahead. For each date we have a cross-section of up

\footnotetext{
${ }^{1}$ Since 1968 a richer data set that includes individual density forecasts is available. To motivate the theory and build our empirical models we opted for the longer time series with only the individual point forecasts.
} 
to 50 forecasts. Our interest in this specific survey is motivated by the fact that it spans the longest time period, an appealing feature since we are trying to capture the properties of a slowly moving component of GDP growth.

The second dataset is given by the Blue Chip Economic Indicators. Each month this dataset provides forecasts up to one and a half years ahead. It is available starting in 1984. The dataset consists entirely of analysts affiliated with the financial services' industry. We merge this dataset with the Livingston Survey by restricting our attention to the two quarters ahead forecasts of real GDP growth rates made in June and December of each year. This has at least two benefits: it increases the cross-sectional size for part of our sample, and it compensates for the drop in the number of analysts that the Livingston Survey experiences in the late 1990's and that continues for about five years. By combining the two datasets, we have an average cross-sectional size in the order of 70 , which allows for a greater degree of precision in the estimation of the cross-sectional moments.

Time varying moments. Figure 2.1 reports the time series for the first three cross-sectional moments of the distribution of expected GDP growth rates. ${ }^{2}$ The figure shows that these moments are varying over time. While average expected GDP growth is on average positive, the skewness is typically negative. The dispersion of the forecasts appears to be very persistent. Quite interestingly, the three moments appear to be mutually uncorrelated. This suggests that the asymmetry of the distribution of forecasts may contain additional information about the risk factors in the economy. A qualitative finding that can be appreciated from looking at Figure 2.1 is that skewness tends to turn more negative right before the beginning of recessions, even at times when the mean forecast would otherwise suggest normal growth rates. This effect is particularly apparent for the last two recessions.

Time series regressions. Table 2.1 reports some additional information about the time series properties of the cross-sectional moments of the distribution of average forecasts. Since the cross-sectional size of the Livingston Survey drops from 44 in the second semester of 1998 to 31 in the first semester of 1999, and it reaches a minimum of 19 in 2004, we conduct our analysis by combining the Livingston survey up to 1998, and the the Blue Chip survey over their entire sample. In the Online Appendix, we

\footnotetext{
${ }^{2}$ Volatility and in particular skewness estimates may be sensitive to outliers. We therefore also considered the quantile-based measures of volatility and skewness (see e.g. Ghysels et al. (2011) and Ghysels (2014) for details about such measures) to control for the effect of outliers. The results are available on request.
} 
Table 2.1: Time Series Properties of Cross-Sectional Moments

\begin{tabular}{|c|c|c|c|c|c|c|}
\hline & \multicolumn{3}{|c|}{ Persistence } & \multicolumn{3}{|c|}{ VAR analysis } \\
\hline & Mean & Volatility & 3rd Mom ${ }^{1 / 3}$ & Mean & Volatility & 3rd Mom ${ }^{1 / 3}$ \\
\hline Lagged Mean & $\begin{array}{l}0.499 \\
(0.07)\end{array}$ & - & - & $\begin{array}{c}0.471 \\
(0.056)\end{array}$ & $\begin{array}{c}-0.021 \\
(0.017)\end{array}$ & $\begin{array}{r}-0.025 \\
(0.051)\end{array}$ \\
\hline Lagged Volatility & - & $\begin{array}{c}0.914 \\
(0.057)\end{array}$ & - & $\begin{array}{c}0.312 \\
(0.248)\end{array}$ & $\begin{array}{c}0.877 \\
(0.048)\end{array}$ & $\begin{array}{r}-0.256 \\
(0.136)\end{array}$ \\
\hline Lagged 3rd Mom ${ }^{1 / 3}$ & - & - & $\begin{array}{c}0.307 \\
(0.086)\end{array}$ & $\begin{array}{c}0.436 \\
(0.124)\end{array}$ & $\begin{array}{r}-0.088 \\
(0.037)\end{array}$ & $\begin{array}{c}0.257 \\
(0.074)\end{array}$ \\
\hline
\end{tabular}

Notes - Time series properties of cross-sectional moments. The panel labeled "Persistence" reports the estimates of the AR(1) coefficients for the mean, volatility, and third centered moment to the power of $1 / 3$. The panel labeled "VAR analysis" reports the estimates of time series regressions of each variable on the corresponding column and the three lagged variables reported in the rows. The numbers in brackets underneath each estimate are heteroskedasticity adjusted standard errors.

conduct the analysis on five alternative datasets: the results are robust across data configurations (see Table A.1 in the Online Appendix).

In the panel labeled "Persistence", we estimate three separate $\operatorname{AR}(1)$ processes for the mean, the volatility, and the third centered moment to the power of $1 / 3$. We choose to focus on this specific power of the third moment, because the model that we propose in the later sections directly imposes restrictions on its dynamics. Our time series estimates suggest that all three moments feature statistically significant first order autocorrelations. The persistence appears to be more pronounced for the first two moments.

The panel labeled "VAR Analysis" further investigates the dynamics by including the lags of all three cross-sectional moments as right hand side variables of the regressions. The interesting finding is that the third moment seems to have predictive power for the conditional mean. More specifically our estimates indicate that following periods of positive asymmetry, the conditional mean increases. This property of the conditional skewness will prove itself important in our theoretical analysis, as news to the shape of the distribution of forecasts will matter in forming the entire stream of future growth prospects. 


\subsection{Predictive regressions}

We explore the predictive content of the first three cross-sectional moments of GDP growth forecasts for equity excess returns, measured as the logarithmic returns on the S\&P500 index minus the returns on three months Treasury bills. Equity prices are obtained from Robert Shiller's website, while Treasuries are obtained from the web site of the Federal Reserve Bank of St. Louis. ${ }^{3}$

Predictive regressions. Table 2.2 reports the results of our predictive regressions. In all the specifications, we regressed the ex-post six months excess returns on the ex ante cross-sectional moments of the distribution of real GDP growth rate, and on some additional variables that are known to have predictive power for equity returns. In the table, $E[$ growth $]$ stands for the median forecast, $V[$ growth $]$ stands for the interquartile measure of volatility (i.e. the $75^{\text {th }}$ percentile less the $25^{\text {th }}$ percentile), and $S[$ growth $]$ is the quantile-based measure of skewness (i.e. the $75^{\text {th }}$ percentile plus the $25^{\text {th }}$ percentile minus twice the median). Part of our results confirm the findings of Campbell and Diebold (2009), in that positive average expected GDP growth rates significantly forecast lower future returns, while the opposite is true for the measure of dispersion of forecasts. Furthermore, while the coefficient on average expected growth is always strongly statistically significant, the one on the dispersion is typically not.

Panel A in Table 2.2 covers cross-sectional moments at the beginning of each six months interval from 1952 to 2010, using the cross-sectional moments of the distribution of real GDP growth rate in addition to the following predictors: cay, the term premium, the dividend yield, and the default spread taken from Lettau and Ludvigson (2001). The latter are variables known to have predictive power for equity returns. Panel B adds a number of recently proposed predictive regressors, which are only available for a shorter sample from 1990 to 2010, namely the VIX ${ }^{2}$, the variance risk premium (denoted VRP) and realized variance of the S\&P 500 (denoted RV) are from Bollerslev et al. (2009). ${ }^{4}$ It also contains the Fear index constructed by Bollerslev and Todorov (2011) which starts in 1996.

\footnotetext{
${ }^{3}$ The dataset is available at http://www.econ.yale.edu/ shiller/data/chapt26.xls.

${ }^{4}$ The VRP series are computed under the assumption of a random walk model for realized volatility. It is worth noting, however, as correctly noted by Bekaert and Hoerova (2014), that alternative RV forecasts not only affect the VRP but also have implications on the role of the latter variable in predicting stock returns.
} 


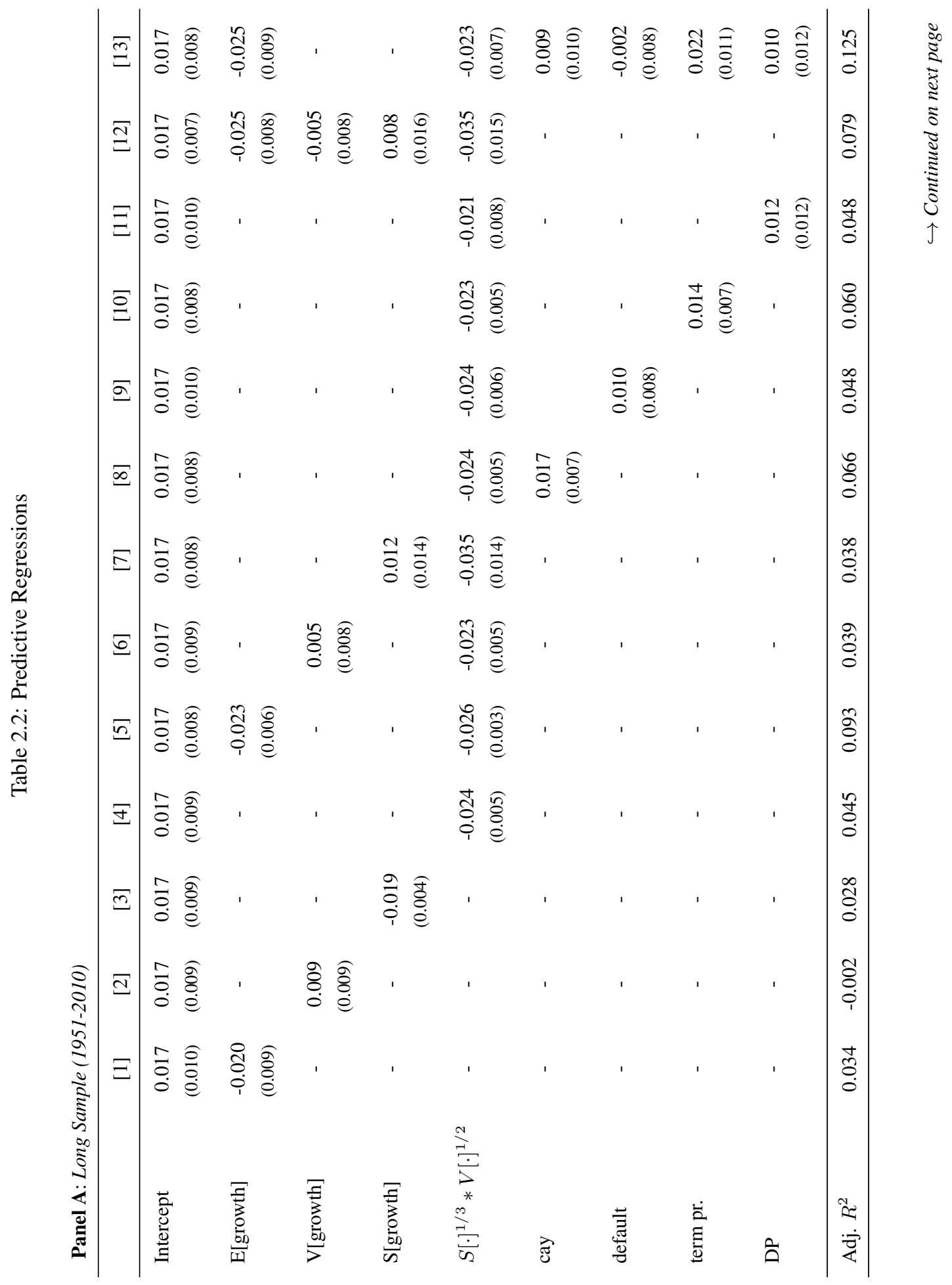




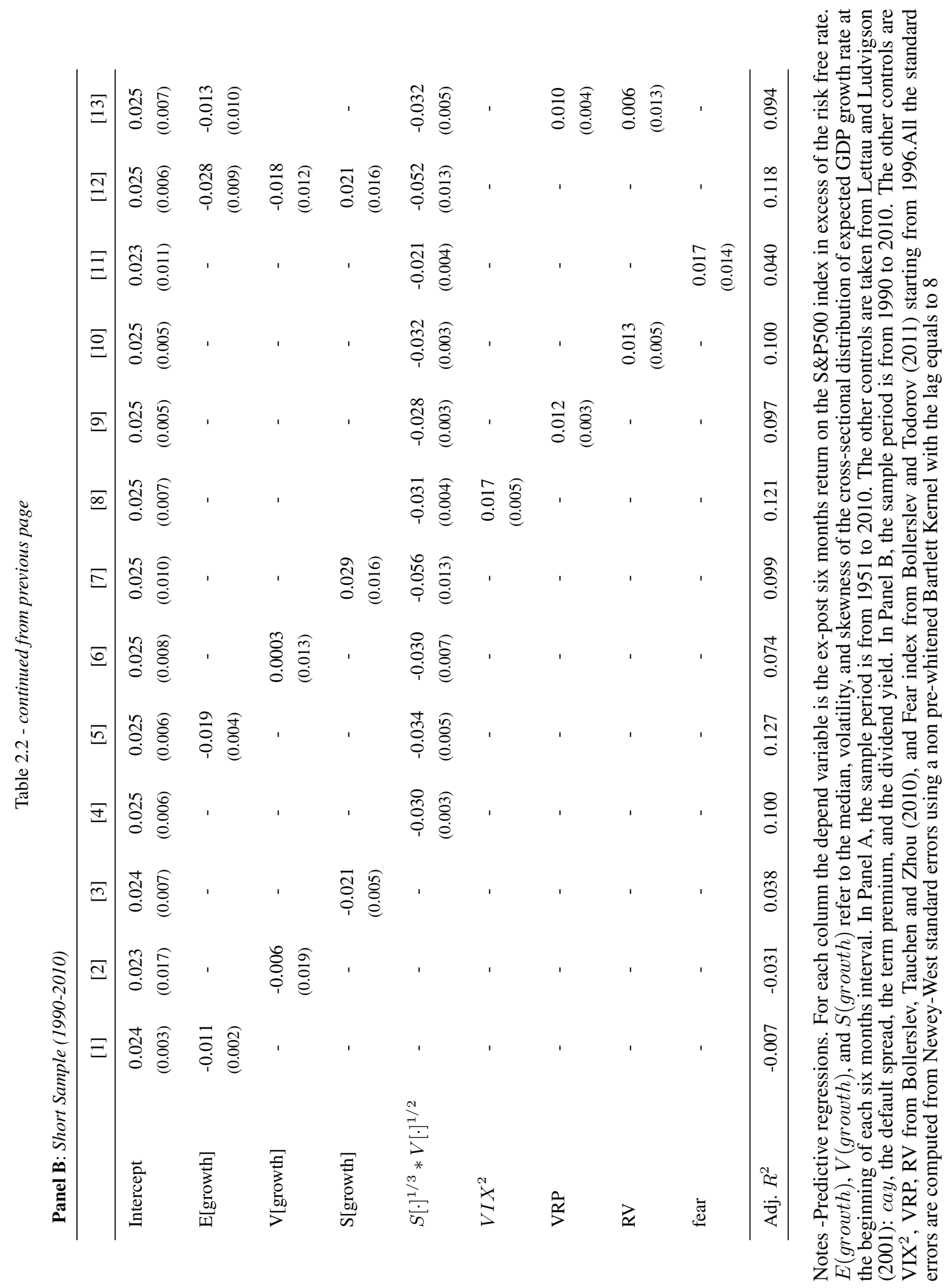


Table 2.3: Correlation between Predictors

\begin{tabular}{|c|c|c|c|c|c|c|c|c|c|c|}
\hline & V[growth] & S[growth] & cay & default & term pr. & DP & VIX $^{2}$ & VRP & RV & fear \\
\hline$\overline{\text { E[growth] }}$ & -0.352 & -0.148 & -0.044 & -0.254 & 0.247 & -0.263 & -0.649 & -0.206 & -0.583 & -0.237 \\
\hline V [growth] & & -0.076 & -0.122 & 0.109 & -0.278 & 0.616 & 0.622 & 0.159 & 0.576 & 0.231 \\
\hline $\mathrm{S}$ [growth] & & & 0.044 & 0.087 & -0.013 & -0.105 & 0.346 & 0.204 & 0.265 & 0.168 \\
\hline cay & & & & -0.100 & 0.274 & 0.006 & -0.133 & 0.228 & -0.248 & 0.353 \\
\hline default & & & & & 0.149 & 0.301 & 0.814 & -0.046 & 0.875 & 0.418 \\
\hline term pr. & & & & & & -0.290 & 0.134 & 0.044 & 0.120 & 0.434 \\
\hline DP & & & & & & & 0.006 & -0.155 & 0.080 & 0.241 \\
\hline VIX $^{2}$ & & & & & & & & 0.325 & 0.893 & 0.510 \\
\hline VRP & & & & & & & & & -0.134 & 0.427 \\
\hline RV & & & & & & & & & & 0.309 \\
\hline
\end{tabular}

Notes - The table reports the correlation between equity return predictors. $E[$ growth], $V[$ growth $]$, and $S[$ growth $]$ refer to the median, volatility, and skewness of the cross-sectional distribution of expected GDP growth rate at the beginning of each six months interval from 1952 to 2010. The predictors cay, the default spread, the term premium, and the dividend yield are from Lettau and Ludvigson (2005), from 1952 to 2010. VIX ${ }^{2}$, VRP and RV are from Bollerslev, Tauchen and Zhou (2010), from 1990 to 2010. Fear is from Bollerslev and Todorov (2011) starting in 1996.

Table 2.3 reports the correlations across the set of variables that we include in our predictive regressions. The first striking observation is the relatively low correlation between $S[$ growth $]$ and the commonly used predictors, namely less than $10 \%$ with cay, the default spread, the term premium and marginally higher with the dividend yield. The correlations are higher but still moderate with the recently proposed risk measures. In particular with $\mathrm{VIX}^{2}$ the correlation is the highest at almost $35 \%$. Interestingly, other measures pertaining to downside risk, such as the Fear index yield only a low correlation of $17 \%$. The latter is important to recognize as it shows that $S[$ growth $]$ is measuring both upside as downside skewness - as indicated in Figure 2.1. In contrast, $V$ [growth] features much stronger correlations with dividend yields and volatility related predictors. Moreover, the conditional mean $E[$ growth $]$ also has strong correlations with the standard regressors and surprisingly large correlations with VIX ${ }^{2}$ and RV. Last, but not least it is also worth noting from Panel A in Table 2.1 that among the three survey-based moment measures $S[$ growth $]$ features the lowest persistence, which is relevant for the econometric analysis of predictive regressions.

For the longest historical data set, we find in Panel A of Table 2.2 that both $E[$ growth $]$ and $S[$ growth $]$ significantly predict stock returns at a six month horizon according to columns [1]-[3]. Note also that $V[$ growth $]$ is not significant and that the conditional mean yields the highest adjusted $R^{2}$. The fact that skewness has predictive power for future equity returns is intriguing. The negative sign of the regression coefficient is also intuitive: a more negative asymmetry suggests an increase in 
tail risk, and equity holders require extra compensation for it. Conversely, a right-skewed asymmetry lowers the equity risk premium - hence the importance of the role played both by left and right skewness. While it is clear from column [2] that the cross-sectional variance is not significant, it is worth noting - as the result in column [4] indicates - that the interaction term $S[\text { growth }]^{1 / 3} * V[\text { growth }]^{1 / 2}$ is significant. The new theoretical asset pricing models discussed later in the dissertation will feature this term. The fact that it is empirically significant will be an implication supported by the data. As indicated in column [5] we also find that the interaction term combined with the cross-sectional first moment yields the best fit and improves the significance of both predictors. In contrast, when the interaction term is combined with either $V[$ growth $]$ or $S[$ growth $]$ we find that it is the interaction term which matters. The results in column [12] further validate this with a regression model which includes all regressors discussed so far.

How does this evidence stand up against other traditional future return predictors? Columns [8] through [11] and [13] indicate that skewness remains significant when traditional return predictors are either added one by one, or jointly to the specification of the predictive regressions.

Panel B of Table 2.2 documents that the results get even stronger if we include predictors proposed in recent years - although they only apply to shorter samples due to data availability. It is noteworthy that the Fear index becomes insignificant when paired with $S[\text { growth }]^{1 / 3} * V[\text { growth }]^{1 / 2}$. While $\mathrm{VIX}^{2}$, VRP and RV remain significant, the latter two become insignificant when combined in a regression with the skewness term, as reported in column [13]. It should also be noted that the best model in Panel B only involved survey-based cross-sectional moments - i.e. the model appearing in column [5]. Finally, as reported in section A.8 of the Online Appendix we find similar results when we pool all the Livingston and Blue Chip survey data.

Taken together, these findings seem to suggest that the odd moments of the distribution of GDP growth forecasts matter in predicting future equity returns. The challenge is to find an asset pricing model which is consistent with such empirical results. 


\section{CHAPTER 3: THE MODEL}

In this chapter we introduce our model with time-varying skewness. Our model features a representative agent that uses the cross-section of analysts' forecasts to discipline her predictions about the conditional moments of expected GDP growth rates. This chapter consists of two parts. First, we analyze a version of the model without any exogenous time variation in volatility. This version of the model can be solved in closed form, and it allows us to highlight some of the key features pertaining the introduction of skewness in a consumption based asset pricing model. In the second half of this chapter, we relax the assumption of no time variation in volatility. In the context of this generalized version of the model, we perform a number of exercises that document the relevance of the interaction between volatility and skewness.

\subsection{A model with time-varying mean and skewness}

Preferences. A representative agent maximizes her lifetime utility, which is defined recursively as

$$
U_{t}=(1-\delta) \log C_{t}+\frac{\delta}{1-\gamma} \log E_{t}\left[\exp \left\{(1-\gamma) U_{t+1}\right\}\right]
$$

where $C_{t}$ stands for consumption at date $t, \gamma$ measures the relative risk aversion (henceforth RRA), and $\delta$ is the subjective discount factor. They correspond to Epstein and Zin (1989) preferences for the case of unit intertemporal elasticity of substitution (henceforth IES). ${ }^{1}$ We choose to restrict our attention to the case of unit IES for multiple reasons. First of all, empirical estimates of the IES, as surveyed by Havranek (2014), are typically around this value. Second, we can derive closed form solutions for the models presented in this section, when preferences are described by equation (3.1). Additionally,

\footnotetext{
${ }^{1}$ These preferences are often denote as "risk sensitive preferences", and they have a long tradition in the macro-finance literature (see among others Hansen and Sargent (1995), Tallarini (2000), Anderson (2005), and Colacito (2008)).
} 
Colacito and Croce (2013) have shown that in a general equilibrium multi-country economy an EIS close to 1 is needed in order to account for several stylized facts of international asset prices and quantities.

The main departure from the constant RRA case often analyzed in the literature lies in the fact that these preferences allow agents to be risk averse in future utility as well as future consumption. The extent of such utility risk aversion depends on the preference for early resolution of uncertainty measured by $\gamma-1>0$. To better highlight this feature of the preferences, it is convenient to look at a third order expansion of the utility function yields ${ }^{2}$

$$
U_{t} \approx(1-\delta) \log C_{t}+\delta E_{t}\left[U_{t+1}\right]+\frac{\delta(1-\gamma)}{2} V_{t}\left[U_{t+1}\right]+\frac{\delta(1-\gamma)^{2}}{6} E_{t}\left[\left(U_{t+1}-E_{t}\left[U_{t+1}\right]\right)^{3}\right]
$$

This approximation, which we use only for the purpose of characterizing the economic intuition of the model, highlights several important aspects of our specification. When $\gamma=1$, the agent is utilityrisk neutral and preferences collapse to the standard expected utility case. However, for levels of risk aversion in excess of 1 , our investor cares also about smooth future utility (low $V_{t}\left[U_{t+1}\right]$ ), and she dislikes negative skewness of her future utility profile.

The variance and negative skewness aversion are specific to this type of preferences and they suggest that skewness of future growth prospects may matter through a variety of channels in our economy. In this dissertation, we focus on a class of models in which consumption growth is predictable. In particular, we analyze a specification for the dynamics of the predictable component of consumption growth rates in which news about expected future growth prospects are drawn from a skewed distribution, a prediction that conforms well with the empirical evidence reported in chapter 2 .

Endowments. The investor is endowed with a stochastic supply of the consumption good

$$
\Delta y_{t+1}=\mu_{c}+x_{t}+\sqrt{\bar{\sigma}} \varepsilon_{t+1}^{c}, \quad \varepsilon_{t+1}^{c} \sim N(0,1)
$$

where $\mu_{c}$ is the unconditional mean of $\Delta y_{t+1}, \bar{\sigma}$ denotes the conditional variance of $\Delta y_{t+1}$, and $x_{t}$ is

\footnotetext{
${ }^{2}$ See section A.10.2 of the Appendix for the details of the derivation.
} 
modeled as a highly persistent $\mathrm{AR}(1)$ process,

$$
x_{t+1}=\rho_{x} x_{t}+\varphi_{e} \sqrt{\bar{\sigma}} \varepsilon_{t+1}^{x} .
$$

We assume that the sequence of shocks $\varepsilon_{t+1}^{x}$ is $i . i . d$. distributed as a Skew-Normal with shape parameter $\phi_{t+1}$. Specifically, the probability distribution function of $\varepsilon_{t+1}^{x}$ is

$$
2 \cdot \underbrace{\frac{1}{\sqrt{2 \pi}} \exp \left\{-\frac{\left(\varepsilon_{t+1}^{x}\right)^{2}}{2}\right\}}_{\text {Normal pdf }} \cdot \underbrace{\int_{-\infty}^{\frac{\phi_{t+1}}{\sqrt{1-\phi_{t+1}^{2}}} \varepsilon_{t+1}^{x}} \frac{1}{\sqrt{2 \pi}} \exp \left\{-\frac{(t)^{2}}{2}\right\} d t}_{\text {Normal CDF }}
$$

In words, the probability of $\varepsilon^{x}$ corresponds to the probability associated to a standard normal distribution tilted by shaping factor, which depends on $\phi$. When $\phi=0$, the standard normal distribution attains. When $\phi>0\left(\phi<0\right.$, respectively), the probability of positive (negative) realizations of $\varepsilon^{x}$ is overweighted (underweighted) relative to the probability of negative (positive) realizations of the shock. This effect is due to the cumulative distribution function being monotonically increasing in its argument, and it generates time-varying skewness in the distribution of the shock $\varepsilon^{x}$, as long as the parameter $\phi$ varies over time. We model the dynamics of $\phi_{t+1}$ as an $\operatorname{AR}(1)$ process:

$$
\phi_{t+1}=\rho_{\phi} \phi_{t}+\sqrt{\sigma_{\phi}} \varepsilon_{t+1}^{\phi}, \quad \varepsilon_{t+1}^{\phi} \sim N(0,1)
$$

where $\rho_{\phi}$ measures the first order autocorrelation of $\phi_{t+1}$, and $\sigma_{\phi}$ is the conditional variance of $\phi_{t+1}$. This distribution allows for values of skewness which are bounded between -1 and 1 . Garcia and Manzanares (2007) employ the skew-normal to analyze the empirical distribution of European professional forecasts of macroeconomic variables.

Financial Markets and Equilibrium. We assume that there is a complete set of state and date contingent securities. In equilibrium, the agent consumes her endowment: $\Delta c_{t+1}=\Delta y_{t+1}$. The price $P_{t}$ of any asset associated to the sequence of stochastic cash flows $\left\{D_{j}\right\}_{j=t}^{\infty}$ is determined by the Euler equation $P_{t}=E_{t}\left[M_{t+1}\left(P_{t+1}+D_{t+1}\right)\right]$, where $M_{t+1}$ is the unique stochastic discount factor (henceforth SDF) that satisfies

$$
M_{t+1}=\frac{\partial U_{t} / \partial C_{t+1}}{\partial U_{t} / \partial C_{t}}
$$


SDF and equity returns. In Appendix A.3, we document that the Campbell and Shiller (1988) approximation yields a very tractable solution of the model. We show that the logarithm of the SDF can be written as

$$
m_{t+1}-E_{t} m_{t+1}=-\left(1-\frac{1}{\theta}\right) \sqrt{\bar{\sigma}} \varepsilon_{t+1}^{c}+\frac{V_{\phi} \sqrt{\sigma_{\phi}}}{\theta} \varepsilon_{t+1}^{\phi}+\frac{B \varphi_{e} \sqrt{\bar{\sigma}}}{\theta} \varepsilon_{t+1}^{x}
$$

where

$$
B=\frac{\delta}{1-\delta \rho_{x}}, \quad V_{\phi}=\frac{0.8 \varphi_{e} B \delta \rho_{\phi}}{1-\delta \rho_{\phi}} \sqrt{\bar{\sigma}}, \theta=\frac{1}{1-\gamma}
$$

We will typically calibrate the preference parameter $\gamma$ to be in excess of 1 . This means that, as long as the dynamics of $x_{t}$ and $\phi_{t}$ are stationary (i.e. $\rho_{x}<1$ and $\rho_{\phi}<1$ ), the loadings on $\varepsilon_{t+1}^{\phi}$ and $\varepsilon_{t+1}^{x}$ in equation (3.5) are going to be negative. This highlights that securities that pay-off in times of high economic growth (large $\varepsilon^{c}$ ), high expected growth (large $\varepsilon^{x}$ ), or high skewness of expected growth (large $\varepsilon^{\phi}$ ) will have large expected returns.

We further investigate the relationship between equity risk premia and skewness, by calculating the returns associated to the stochastic cash flow

$$
\Delta d_{t}=\mu_{c}+\lambda\left(x_{t-1}+\sqrt{\bar{\sigma}} \varepsilon_{t}^{c}\right)+\sqrt{\sigma_{d}} \sqrt{\bar{\sigma}} \varepsilon_{t}^{d}, \quad \varepsilon_{t}^{d} \sim N(0,1)
$$

Absent the shock $\varepsilon_{t}^{d}$, the dividend process would consist of a claim to levered consumption. ${ }^{3}$ We introduce the shock $\varepsilon_{t}^{d}$ to break the otherwise perfect correlation between consumption and dividend growth rates. We document our calibration below.

We show in section A.2 of the Technical Appendix that the conditional equity risk premium is equal to

$$
E_{t}\left[r_{t+1}^{d}-r_{t}^{f}\right]=\bar{r}-\alpha \frac{K_{1}(\lambda-1)}{1-K_{1} \rho_{x}} \varphi_{e} \sqrt{\bar{\sigma}} \rho_{\phi} \cdot \phi_{t}
$$

\footnotetext{
${ }^{3}$ We restrict the conditional mean of consumption and dividend growth rates to be the same to be consistent with balanced growth.
} 
where $\alpha>0$ is related to the slope of the normal cdf, $K_{1} \in(0,1)$ is the slope of the Campbell and Shiller (1988) approximation and all other parameters are defined in the setup of the economy. ${ }^{4}$ Equation (3.6) documents an important feature of our model. The degree of skewness of expected economic growth, indexed by $\phi_{t}$, predicts future equity returns. In particular, times of positive (negative) skewness are associated with a decline (rise) in expected returns. In section A.14 of the Appendix we document that this result is robust to a variety of alternative approximations and numerical solutions.

\subsection{A model with time-varying volatility and skewness}

In this section, we are interested in better understanding the interaction between time-varying skewness and time-varying volatility, which plays a key role in the predictive regressions presented in chapter 2. To this end, we generalize our baseline model, by introducing an exogenous process for volatility.

Setup of the model. We modify the setup of the model presented in the previous section by replacing the constant variances (denoted as $\bar{\sigma}$ ) in equations (3.2) and (3.3) with the following time-varying variance process:

$$
\sigma_{t}=\bar{\sigma}\left(1-\rho_{\sigma}\right)+\rho_{\sigma} \sigma_{t-1}+\sqrt{\sigma_{\epsilon}} \varepsilon_{t}^{\sigma}, \quad \varepsilon_{t}^{\sigma} \sim N(0,1)
$$

where $\bar{\sigma}$ denotes the unconditional mean of the volatility process, $\rho_{\sigma}$ measures its persistence, and $\sigma_{\epsilon}$ is the conditional mean of $\sigma_{t}$. Equation (3.7) allows us to incorporate time-varying economic uncertainty in the dynamics of the model. We use this autoregressive specification because it allows us to compare the performance of our model with some of the related models in this literature, including Bansal and Yaron (2004) and Drechsler and Yaron (2011) (see chapter 4 for this analysis).

\footnotetext{
${ }^{4}$ In section A.14.1 of the Appendix we document that the coefficient in front of $\phi_{t}$ is always negative for several alternative approximations.
} 
Calibration. We use GMM to estimate the parameters governing the dynamics of $x_{t}, \sigma_{t}$, and $\phi_{t}$. Specifically, we define

$$
\begin{aligned}
\widehat{E}_{t}^{c s}\left(\Delta c_{t+1}\right) & =\frac{1}{n} \sum_{i=1}^{n} E_{t}^{i}\left(\Delta c_{t+1}\right) \\
\widehat{V}_{t}^{c s}\left(\Delta c_{t+1}\right) & =\frac{1}{n} \sum_{i=1}^{n}\left[E_{t}^{i}\left(\Delta c_{t+1}\right)-\widehat{E}_{t}^{c s}\left(\Delta c_{t+1}\right)\right]^{2} \\
\widehat{S}_{t}^{c s}\left(\Delta c_{t+1}\right) & =\frac{\frac{1}{n} \sum_{i=1}^{n}\left[E_{t}^{i}\left(\Delta c_{t+1}\right)-\widehat{E}_{t}^{c s}\left(\Delta c_{t+1}\right)\right]^{3}}{\left(\widehat{V}_{t}^{c s}\left(\Delta c_{t+1}\right)\right)^{3 / 2}}
\end{aligned}
$$

as the cross-sectional mean, variance, and skewness of the distribution of analysts' forecasts. We estimate paramaters by minimizing the distance between the means, the variances, and the autocorrelations of the processes in equations (3.3), (3.4), and (3.7) and the cross-sectional moments in (3.8). ${ }^{5}$ In the interest of space, all details are reported in Appendix A.5.

The estimated parameters are presented in Table 3.1 along with the associated standard errors. The column labeled "Model" reports the coefficients that we use for our baseline calibration, and it documents that they are all well within the conventional confidence intervals of the estimated parameters. Additionally, we calibrate the preference parameters $\gamma$ and $\delta$ to 10 and 0.993 , respectively. These numbers are in line with what typically encountered in the asset pricing literature (see inter alia Bansal and Yaron (2004) and Colacito and Croce (2011)). The remaining parameters $\mu_{c}, \varphi_{e}, \lambda$, and $\sigma_{d}$ are calibrated to target average growth rates of consumption and dividend of $1.6 \%$ per year, a moderate degree of first order autocorrelation of consumption and dividend growth rates $(0.05$ and 0.1 , respectively), and a less than perfect correlation between consumption and dividend growth rates (0.65 in our model). ${ }^{6}$

${ }^{5}$ We note that the process $\phi_{t}$ is related to the cross-sectional skewness $\widehat{S}_{t}^{c s}$ through the transformation $\frac{\widehat{A}_{t}^{c s}}{\sqrt{K_{\phi}^{2}+2\left(\widehat{A}_{t}^{c s}\right)^{2} / \pi}}$, where $\widehat{A}_{t}^{c s}=\left[\frac{E_{t}\left(x_{t+1}-E_{t}\left(x_{t+1}\right)\right)^{3}}{V_{t}\left(x_{t+1}^{3 / 2}\right)}\right]^{1 / 3}$.

${ }^{6}$ In the data the point estimate of the correlation between consumption and dividend growth is 0.36 with a standard error of 0.2 over the sample for which we have analysts' forecasts. Bansal and Yaron (2004) report a point estimate of 0.55 with a standard error of 0.34 , obtained using observations from 1929 to 1998 . Our calibrated correlation is well within the confidence regions of these empirical estimates. 
Table 3.1: Calibration

\begin{tabular}{clcc}
\multicolumn{2}{c}{ Panel A: dynamics of mean, variance, and skewness } & & \\
\hline Parameter & Description & Model & GMM \\
\hline$\rho_{x}$ & AR coefficient of the expected consumption growth rate & 0.690 & 0.540 \\
$\bar{\sigma}$ & Unconditional variance of the short-run shock & $3.13 \times 10^{-4}$ & $3.85 \times 10^{-4}$ \\
$\sqrt{\sigma_{\varepsilon}}$ & Conditional volatility of the variance of the short-run shock & $1.50 \times 10^{-4}$ & $\left.2.81 \times 10^{-5}\right)$ \\
$\rho_{\sigma}$ & AR coefficient of the variance of the short-run shock & 0.464 & $\left(4.89 \times 10^{-5}\right)$ \\
$\sqrt{\sigma_{\phi}}$ & Conditional volatility of skewness & 0.407 & $(0.075)$ \\
$\rho_{\phi}$ & AR coefficient of skewness & 3.644 & $(1.796)$ \\
& & & 0.185 \\
\hline
\end{tabular}

Panel B: other parameters

\begin{tabular}{clc}
\hline Parameter & Description & Model \\
\hline$\gamma$ & Risk aversion & 10 \\
$\delta$ & Subjective discount factor & 0.993 \\
$\mu_{c}$ & Average consumption growth & 0.008 \\
$\varphi_{e}$ & Ratio of long-run shock and short-run shock volatilities & 0.200 \\
$\lambda$ & Leverage coefficient & 4.500 \\
$\sigma_{d}$ & Scale parameter of dividends' volatility & 28 \\
\hline
\end{tabular}

Notes - Panel A reports the calibration of the parameters associated to the transition dynamics of $x_{t}, \sigma_{t}$, and $\phi_{t}$ (column labeled "Model"), along with the GMM estimated values (column labeled "GMM"). The numbers in parenthesis are the standard errors of the estimated coefficients. Panel B reports the calibration of all the remaining parameters of the model. The calibration is set to semi-annual frequency.

Interaction between skewness and volatility. A key feature of the stochastic discount factor with recursive preferences is that it reflects news to the future wealth of the representative agent. Figure 3.1(a) shows the utility function, a measure of agents' wealth, as function of $\sigma_{t}$ and $\phi_{t}$. This utility can take on a large range of values as the conditional skewness and volatility explore the state space. Figure 3.1(b) reports three horizontal cuts of the utility function. The middle line refers to the case of zero skewness. This is a version of the Bansal and Yaron (2004) model. Notice that in this case utility is not extremely sensitive to changes in volatility, given that our chosen calibration postulates a very small amount of time-varying volatility. The situation is very different for the cases in which skewness is positive or negative. The interaction between second and third moments produces larger movements in total discounted utilities. As the degree of asymmetry becomes more and more positive, volatility is welfare increasing as it implies a larger probability of landing in an extremely good state of the economy. The opposite is true for the case of negative skewness, since more volatility increases the likelihood of a left tail event. The interaction between time-varying volatility and skewness is 

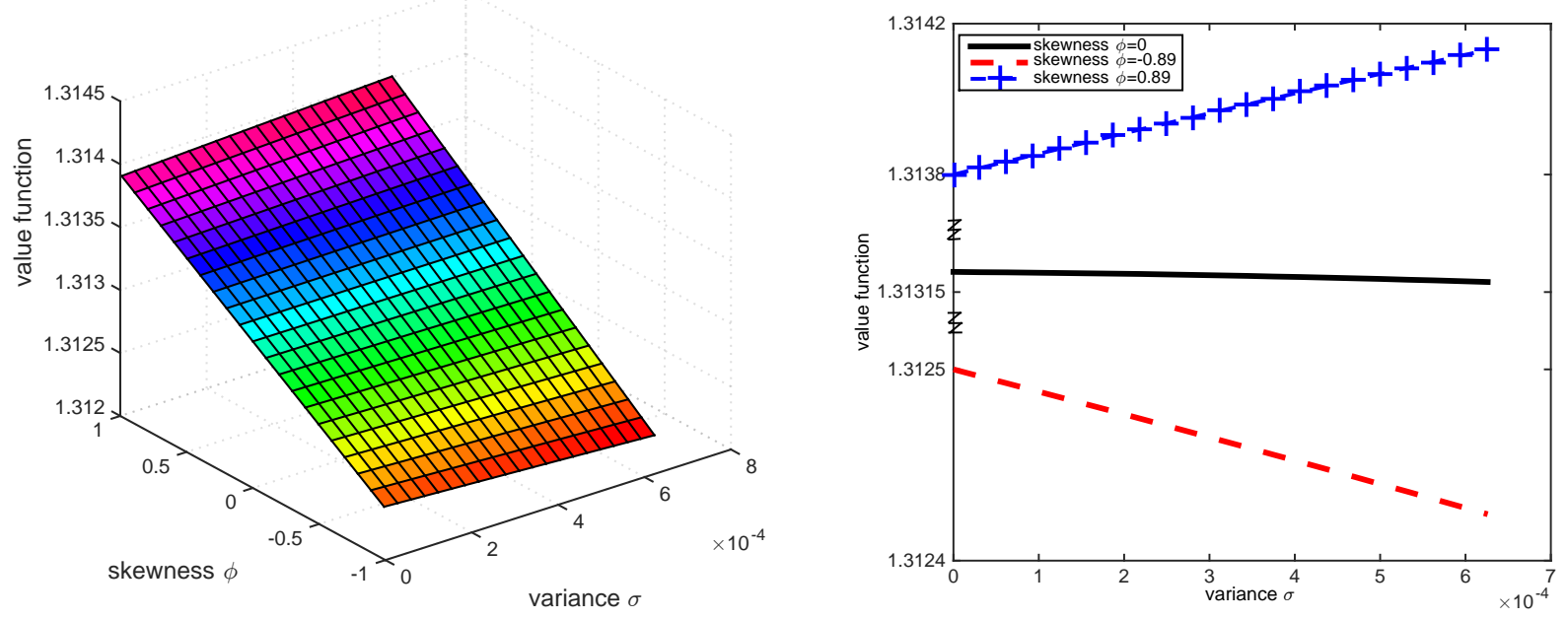

Figure 3.1: Utility function. The left panel reports the utility function, $\widetilde{V}_{t}=V_{t}-x_{t}$, as a function of the skewness and of the variance parameters. The right panel shows three slices of $\widetilde{V}_{t}$ for different values of skewness.

therefore an important determinant of risk in this economy.

Quantitative performance. We perform two exercises to assess the performance of our model. The first one consists in replicating the type of regressions presented in chapter 2 , in the context of our model. Specifically, we simulate our model using the calibration reported in Table 3.1 and regress the excess returns on equity onto the lagged values of $x_{t}, \sqrt{\sigma_{t}}, \sigma_{t}, \phi_{t}, \phi_{t}^{2}$, and $\sqrt{\sigma_{t}} \phi_{t}$. Table 3.2 presents our results.

The table documents that not only the model produces the right signs for all the coefficients of interest, but also that the magnitudes are very close to the ones that we estimated in the data. Since the log-linear approximation of the model suggests that $x_{t}$ should not predict future returns, we report the results with and without including $x_{t}$ as a regressor. The table shows that the results are unaffected by the inclusion of this variable.

The second exercise consists in feeding directly the time series of the cross-sectional moments of analysts' forecasts into our model. Specifically, we plug the time series of the cross-sectional moments of the distribution of analysts' forecasts in (3.8) into the equation that describes the evolution 
Table 3.2: Model Implied Predictive Regressions at Semi-annual Frequency

\begin{tabular}{|c|c|c|c|c|}
\hline \multirow[b]{2}{*}{$x_{t}$} & \multicolumn{2}{|c|}{ Data } & \multicolumn{2}{|c|}{ Model } \\
\hline & -0.027 & - & -0.001 & - \\
\hline$\sqrt{\sigma}_{t}$ & 0.015 & 0.004 & 0.000 & 0.003 \\
\hline$\sigma_{t}$ & -0.027 & -0.003 & 0.005 & 0.003 \\
\hline$\phi_{t}$ & 0.061 & 0.053 & 0.011 & 0.010 \\
\hline$\phi_{t}^{2}$ & -0.005 & -0.007 & -0.001 & -0.001 \\
\hline$\phi_{t} \sqrt{\sigma}_{t}$ & -0.090 & -0.077 & -0.011 & -0.009 \\
\hline
\end{tabular}

Notes -The columns labeled "Empirical Data" report predictive regressions of ex-post six months return on the S\&P500 index in excess of the risk free rate. $x_{t}, \sigma_{t}, \phi_{t}$ are constructed using the cross-sectional moments of expected GDP growth rate at the beginning of each six months. The columns labeled "Model" report predictive regressions for model implied equity excess returns using semi-annual calibration. The results are obtained from simulating the models 1000 times with sample size equal to 60 years (120 periods). All independent variables are standardized by subtracting their unconditional means and dividing by their standard deviations.

of the conditional risk premium in our model. ${ }^{7}$. We then calculate the correlation between these expected returns predicted by the model and the actual subsequent excess returns in the data. To better characterize the relevance of skewness, we compute the same set of correlations for a version of the model in which any time-variation in skewness has been shut down. Table 3.3 reports the results of our analysis.

We perform this analysis by looking at the five quintiles of the distribution of skewness (the $\phi_{t}$ process). The row labeled "Benchmark" in Panel A of Table 3.3 reports the correlations for the baseline version of our model featuring time-variation in both variance and skewness. The row labeled "No skewness" refers instead to a version of the model with only time-variation in volatility (i.e. the model that attains by replacing equation (3.4) with $\phi_{t}=0, \forall t$ ). A comparison between the two sets of correlations highlights that the model that takes into account time-variation in the asymmetry of expected growth rates performs consistently better than its direct competitor. The row labeled "\% change" documents that correlations can be even more than twice as large in the benchmark version of our model. Furthermore the improvement in correlations is particularly apparent for the more extreme quintiles of the distribution of skewness. This conveys the idea that our model helps refining the forecast of future returns in times in which the degree of asymmetry of expected macroeconomic fundamentals is more pronounced.

\footnotetext{
${ }^{7}$ In the interest of space, we describe all the details is section A.4 of the Technical Appendix.
} 
Table 3.3: Correlation of Excess Returns: Quintile Analysis

Panel A: Correlations by Skewness based Quintiles

\begin{tabular}{lccccc}
\hline & Q1 & Q2 & Q3 & Q4 & Q5 \\
\hline No Skewness & 0.096 & 0.126 & 0.325 & 0.073 & 0.122 \\
Benchmark & 0.219 & 0.155 & 0.327 & 0.092 & 0.141 \\
\% Change & $(128.6 \%)$ & $(23.3 \%)$ & $(0.8 \%)$ & $(26.0 \%)$ & $(16.0 \%)$ \\
\hline
\end{tabular}

Panel B: The Role of Volatility

\begin{tabular}{lccccr}
\hline & Q1 & Q2 & Q3 & Q4 & Q5 \\
\hline Low $\sigma_{t}$ & 0.157 & -0.319 & 0.086 & 0.131 & -0.099 \\
High $\sigma_{t}$ & 0.101 & 0.402 & 0.289 & 0.209 & 0.228 \\
\hline
\end{tabular}

Notes - The table reports the correlations between expected log-excess returns from the model and six months ahead realized excess return on S\&P 500 index in each of the five quinteles of the distibution of skewness. The quintiles are labeled as Q1 through Q5. The time series of $\sigma_{t}$ and $\phi_{t}$ are constructed using the crosssectional moments of the distribution of the growth rate of real GDP expectations from the Livingston and Blue Chips datasets. In Panel A, we report the correlations with the model presented in section 3.2 (labeled as "Benchmark") and the correlations with the same model with the exception of the time-variation in skewness being shut down (label as "No Skewness"). The row label "\% Change" reports the percentage increase in correlations across the two versions of the model. Panel B reports the breakdown of the correlations in Panel A, row labeled "Benchmark" with respect to volatility. The row labeled "Low $\sigma_{t}$ " refers to the sample in which the volatility is below its median value, while the row labeled "High $\sigma_{t}$ " refers to the sample in which the volatility is above its median value. The sample is 1951:1 to 2010:1.

Panel B of Table 3.3 sheds light on the importance of the interaction between volatility and skewness. For each quintile of the distribution of skewness, we decompose the correlation between expected returns in the model and future realized returns into periods of below median volatility of expected growth rates (row label "Low $\sigma_{t}$ ") and periods of above median volatility of expected growth rates (row labeled "High $\sigma_{t}$ "). The table documents that the correlation is typically larger in periods of high volatility. We see this in particular for Q2 through Q5 where the correlations typically more than double when volatility is high. 


\section{CHAPTER 4: COMPARISON WITH OTHER MODELS}

In this chapter we perform a comparison of our model with other existing model in the literature. The common feature of all these models lies in their ability of generating large equity risk premia through tail risk in the postulated consumption and cash flow dynamics. We document, however, that time-varying skewness operates in very different ways across models. This is reflected in their comparative performance as far as the predictive regressions are concerned.

The chapter is organized as follows. First, we document the different behavior of skewness in our model relative to the class of models featuring jumps in volatility (as in Bansal and Shaliastovich (2011) and Drechsler and Yaron (2011)). Second, we document the quantitative performance of all these models for a large set of asset pricing moments, and for the predictive regressions discussed in chapter 2 . We conclude this chapter with a sensitivity analysis of our model.

\subsection{Skewness in consumption based asset pricing models}

We consider a comparison between our model and some alternative ways of introducing time varying skewness in expected consumption growth rates. Specifically, we focus on the class of models with jumps in volatility and in expected consumption growth of the type discussed in Drechsler and Yaron (2011), Bansal and Shaliastovich (2010), and Bansal and Shaliastovich (2011).

All the models that we consider are nested within the following specification for consumption and dividend growth rates and the associated conditional mean and volatility: 
Table 4.1: Monthly Calibration

Panel A: Common Parameters

\begin{tabular}{clc}
\hline Parameter & Description & \\
\hline$\rho_{x}$ & AR coefficient $x_{t}$ & 0.955 \\
$\bar{\sigma}$ & Unconditional variance of $\sigma_{t}$ & $4.62 \times 10^{-5}$ \\
$\rho_{\sigma}$ & AR coefficient of $\sigma_{t}$ & 0.93 \\
$\sqrt{\sigma_{\varepsilon}}$ & Conditional volatility of $\sigma_{t}$ & $5.10 \times 10^{-6}$ \\
$\mu_{c}$ & Average consumption growth rate & 0.0008 \\
$\varphi_{e}$ & Scale parameter of long-run volatility & 0.050 \\
$\lambda$ & Leverage coefficient & 3 \\
$\sigma_{d}$ & Scale parameter of dividends' volatility & 20.25 \\
$\gamma$ & Risk aversion & 10 \\
$\delta$ & Subjective discount factor & 0.998 \\
\hline
\end{tabular}

Panel B: Model Specific Parameters

\begin{tabular}{clcc}
\hline Parameter & Description & Jumps & Skew-Normal \\
\hline$\mu_{x}$ & Location parameter of exponential distribution of $\xi_{t}^{x}$ & $3.645 \varphi_{e} \sqrt{\bar{\sigma}}$ & 0 \\
$l_{0}^{x}$ & Constant term of the jump intensity of $J_{t+1}^{x}$ & $0.8 / 12 / \bar{\sigma}$ \\
$l_{1}^{x}$ & Loading on $\sigma_{t}$ for the jump intensity of $J_{t+1}^{x}$ & $2.55 \bar{\sigma}$ \\
$\mu_{\sigma}$ & Location parameter of exponential distribution of $\xi_{t}^{\sigma}$ & 0 & \\
$l_{0}^{\sigma}$ & Constant term of the jump intensity of $J_{t+1}^{\sigma}$ & $0.8 / 12 / \bar{\sigma}$ & 0.470 \\
$l_{1}^{\sigma}$ & Coefficient in front of $\sigma_{t}$ for the jump intensity of $\sigma_{t}$ & & 0.80 \\
$\sqrt{\sigma_{\nu}}$ & Conditional volatility of the skewness process $\nu_{t}$ & & \\
$\rho_{\nu}$ & AR coefficient of the skewness process $\nu_{t}$ & & \\
\hline
\end{tabular}

Notes - The table reports the choice of parameters for the monthly calibration of the model. Panel A shows the calibration of the parameters that are in common between the model with skew-normal innovations and the model with jumps. Panel B shows the remaing model specific parameters. The parameters $\mu_{x}, l_{0}^{x}$, and $l_{1}^{x}$ control the dynamics of the jumps to $x_{t}$. The parameters $\mu_{\sigma}, l_{0}^{\sigma}$, and $l_{1}^{\sigma}$ control the dynamics of the jumps to volatility. The parameters $\rho_{\nu}$ and $\sqrt{\sigma_{\nu}}$ denote the autocorrelation and volatility of the process $\nu_{t}=\phi_{t} / \sqrt{1-\phi_{t}^{2}}$, which controls the dynamics of skewness.

$$
\begin{aligned}
\Delta c_{t+1} & =\mu_{c}+x_{t}+\sqrt{\sigma_{t}} \varepsilon_{t+1}^{c} \\
\Delta d_{t+1} & =\lambda \Delta c_{t+1}+\sqrt{\sigma_{d}} \sqrt{\sigma_{t}} \varepsilon_{t+1}^{d} \\
x_{t+1} & =\rho_{x} x_{t}+\varphi_{e} \sqrt{\sigma_{t}^{x}} \varepsilon_{t+1}^{x}+J_{t+1}^{x} \\
\sigma_{t+1} & =\left(1-\rho_{\sigma}\right) \bar{\sigma}+\rho_{\sigma} \sigma_{t}+\sqrt{\sigma_{\varepsilon}} \varepsilon_{t+1}^{\sigma}+J_{t+1}^{\sigma} \\
\sigma_{t}^{x} & =\sigma_{t}\left(1-2\left(E_{t}\left[\phi_{t+1}\right]\right)^{2} / \pi\right)^{-1}
\end{aligned}
$$

where $\varepsilon_{t+1}^{c}, \varepsilon_{t+1}^{d}$, and $\varepsilon_{t+1}^{\sigma}$ are i.i.d. distributed as standard normal, $\varepsilon_{t+1}^{x}$ is i.i.d. distributed as a 
Skew-Normal,

$$
\varepsilon_{t+1}^{x} \sim S K N\left(0,1, \phi_{t+1}\right)
$$

and $J_{t+1}^{x}$ and $J_{t+1}^{\sigma}$ are modeled as jump processes

$$
\begin{aligned}
J_{t+1}^{x} & =\sum_{j=1}^{N_{t+1}^{x}} \xi_{j, t+1}^{x}, \quad N_{t+1}^{x} \sim \operatorname{Poisson}\left(l_{0}^{x}+l_{1}^{x} \sigma_{t}\right), \quad \xi_{j, t+1}^{x} \sim-\exp \left(\mu^{x}\right)+\mu_{x} \\
J_{t+1}^{\sigma}= & \sum_{j=1}^{N_{t+1}^{\sigma}} \xi_{j, t+1}^{\sigma}, \quad N_{t+1}^{\sigma} \sim \operatorname{Poisson}\left(l_{0}^{\sigma}+l_{1}^{\sigma} \sigma_{t}\right), \quad \xi_{j, t+1}^{\sigma} \sim \exp \left(\mu^{\sigma}\right)-\mu_{\sigma} .
\end{aligned}
$$

Clearly, by setting the skew-normal parameter $\phi_{t}$ equal to 0 for all $t$, the jump dynamics of the Drechsler and Yaron (2011) model attains. Drechsler and Yaron (2011) also consider the case in which the jump size of the $J_{t+1}^{x}$ process is normally distributed. Since this model features by construction a constant skewness, we will abstract from discussing it in the remainder of this section. By setting the jump processes $J_{t+1}^{x}$ and $J_{t+1}^{\sigma}$ to zero delivers the benchmark consumption dynamics presented in this dissertation. Note that in both models, the variance dynamics is entirely accounted by the $\sigma_{t}$ process. We obtain this by adjusting the variance of the conditional mean process, $\sigma_{t}^{x}$, to eliminate the effect of skewness on volatility (last equation of the system in (4.1)).

Table 4.1 reports our baseline calibration. We calibrate the model to describe a monthly decision problem, consistently with the frequency choice of Drechsler and Yaron (2011). We choose to calibrate the parameters that are in common to all models as in Drechsler and Yaron (2011), while the dynamics of the skewness process are chosen in a way to be consistent to the semi-annual moments reported in chapter $3 .^{1}$

Figure 4.1 highlights the very different dynamics of the conditional skewness of expected consumption growth produced by our model and by the model with jumps. Since the model with normally distributed jumps does not feature any time-variation in skewness, we will focus our attention on the model with negative exponential jumps. The figure documents that the two models have sharply

\footnotetext{
${ }^{1}$ The persistence of the process that controls the dynamics of skewness needs to be set to a larger value to reflect the monthly nature of the calibration. To prevent $\phi_{t}$ from hitting its bounds, we specify the AR(1) dynamics on $\nu_{t}=\phi_{t} / \sqrt{1-\phi_{t}^{2}}$, which takes values on the entire real line.
} 


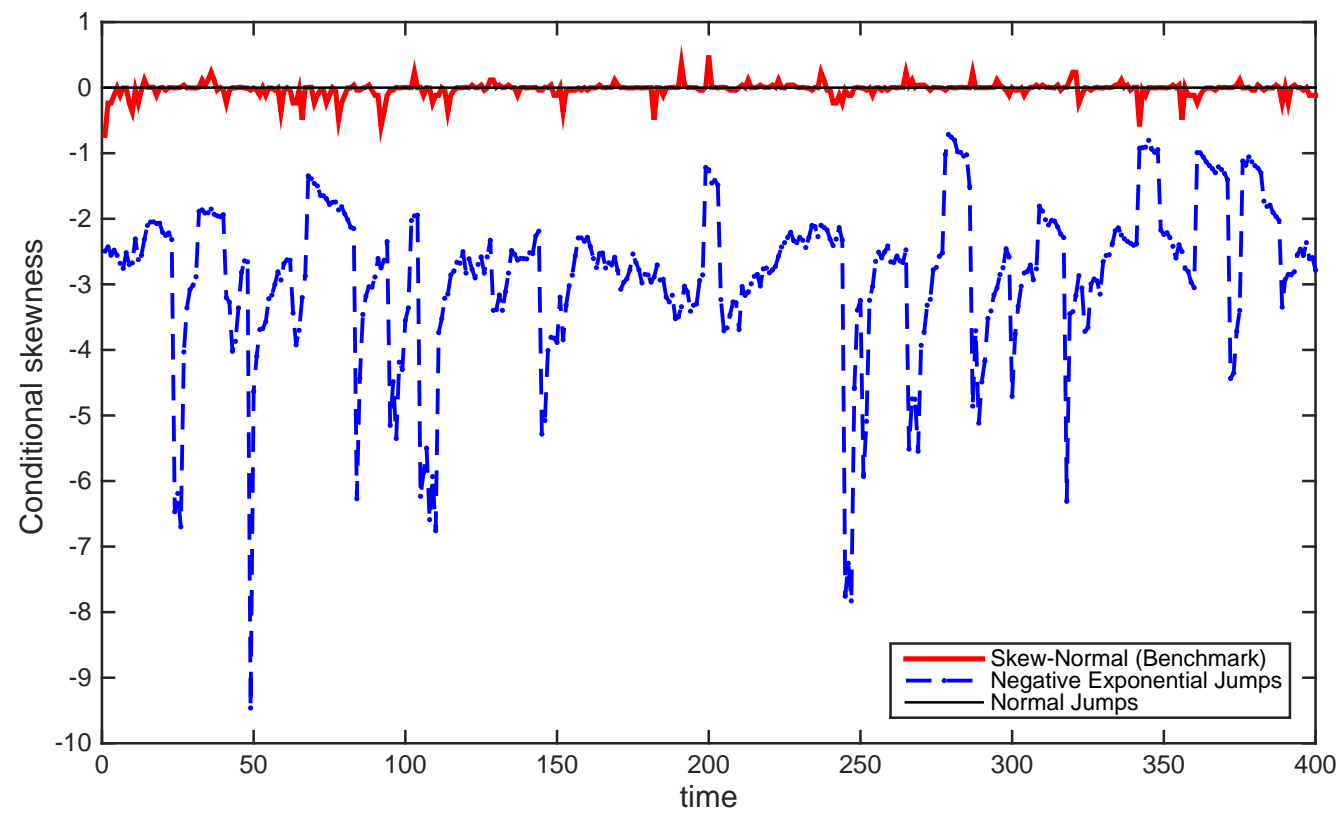

Figure 4.1: Comparison of conditional skewness of expected consumption growth across models. The thick line represents our benchmark model with skew-normally distributed innovations. The dashed line refers to the model with jumps with negative exponential innovations. The dash-dot line (the zero line) is the conditional skewness in the model with normally distributed jumps.

different predictions as far as the range of values of skewness is concerned, as well as its degree of persistence and volatility. Note that our benchmark model appears to more closely resemble the magnitude and extent of time-variation of skewness that we presented in chapter 2.

Furthermore, we can characterize the specific timing of skewness in the model with jumps. Given the affine nature of the model with jumps, it is easy to show that both variance and third central moment of $x_{t}$ in the Drechsler and Yaron (2011) model depend linearly on $\sigma_{t}$. Specifically, we document in Appendix A.6 that

$$
V_{t}\left[x_{t+1}\right]=\left(\varphi_{e}^{2}+l_{1}^{x} \mu_{x}^{2}\right) \sigma_{t}, \quad \text { and } \quad E_{t}\left[\left(x_{t+1}-E_{t}\left[x_{t+1}\right]\right)^{3}\right]=-2 \mu_{x}^{3} l_{1}^{x} \sigma_{t}
$$

This has important implications for our model. First of all, it highlights that variance and skewness do not have independent effects: once equity returns are regressed on the conditional variance of $x_{t}$, the third moment does not have any additional explanatory power. Additionally, the skewness is going to 
be an increasing function of $\sigma_{t}$ :

$$
\text { Skewness }{ }_{t}\left[x_{t+1}\right]=-\frac{2 \mu_{x}^{3} x_{1}^{x}}{\left(\varphi_{e}^{2}+l_{1}^{x} \mu_{x}^{2}\right)^{3 / 2}} \frac{1}{\sqrt{\sigma_{t}}}
$$

This means that equity risk premia are going to be an increasing function of skewness, the same way they are positively related to variance. This finding is generally at odds with our empirical results, and it calls for an additional risk factor that allows us to disentangle variance from skewness. Our model with time-varying Skew-Normal innovations represents one such model.

\subsection{Asset pricing moments}

In Table 4.2 we report several asset pricing moments for our benchmark model, the model with jumps, as well as for a number of alternative specifications. For comparison, we report the actual moments calculated using annual US data from 1950 to 2012 obtained from Robert Shiller's web site in the first column. The results are obtained from simulating the models 1000 times with sample size equal to 100 observations.

Several results ought to be noticed. First of all, a comparison between the specifications in columns 2 and 3, and the specification in column 1 reveals that the introduction of skewness determines a large increase in the average equity risk premium. This increase comes together with more volatile equity excess returns, and substantially larger equity Sharpe ratios. Second, the average risk free rate is almost unaffected by the introduction of skewness dynamics. Its volatility increases in the two skewness calibrations, but the $95 \%$ confidence intervals (reported underneath each estimate) reveal that these increases are well within the margin of significance. Third, the average price-dividend ratio is even closer to the data thanks to the introduction of the time-varying skewness process, and so are its volatility and autocorrelation. Table 4.2 also documents the ability of all the models to replicate the almost null amount of skewness of equity excess returns, risk-free rates, and price-dividend ratios.

The next two columns (labeled 4 and 5) report the asset pricing moments associated to two alternative specifications of our benchmark model model. In column 4, we compensate the change in 


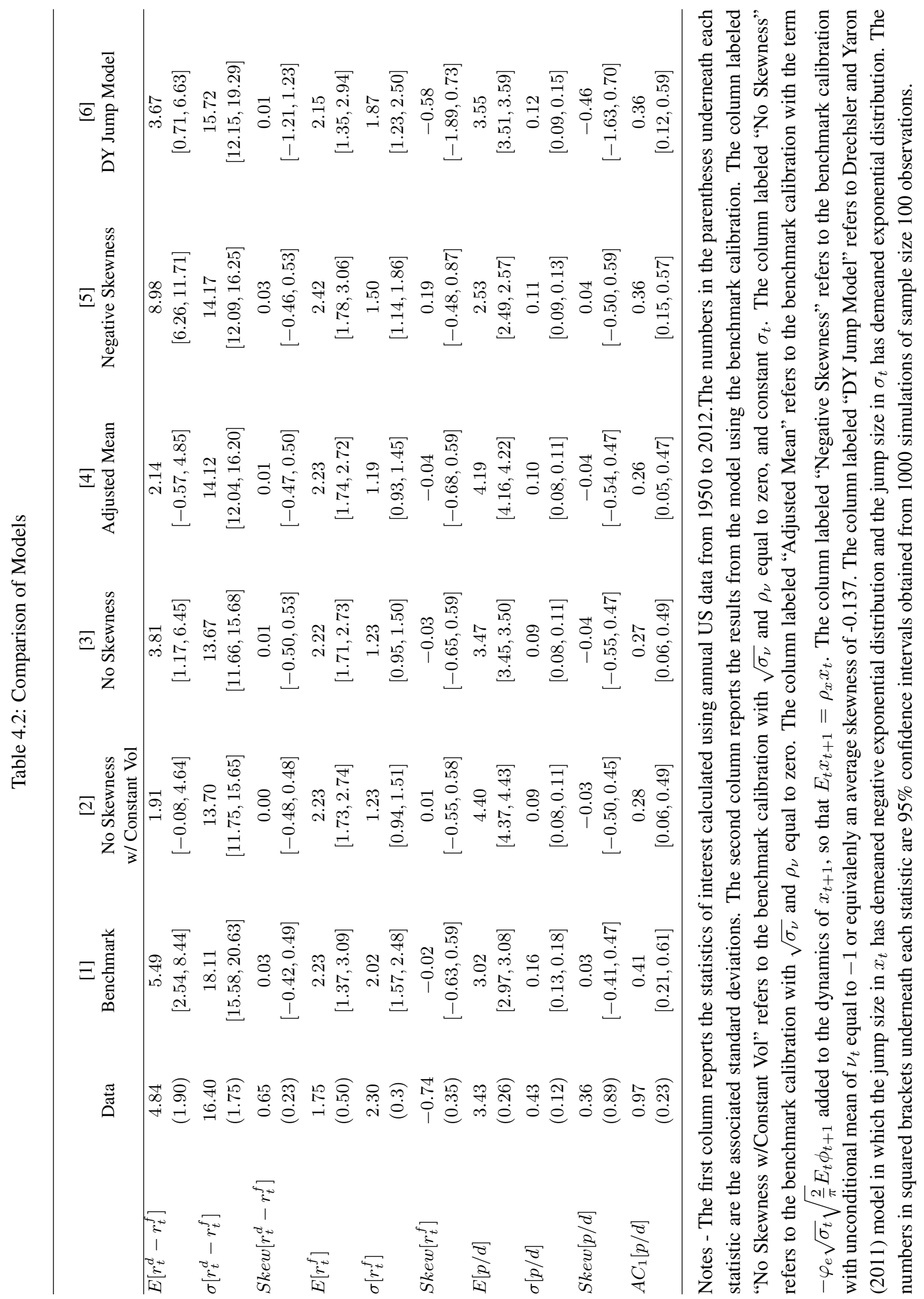


Table 4.3: The Role of Skewness in Predictive Regressions

\begin{tabular}{|c|c|c|c|c|c|c|c|c|c|c|}
\hline & \multicolumn{5}{|c|}{ Benchmark Model } & \multicolumn{5}{|c|}{ Model with jumps } \\
\hline & {$[1]$} & {$[2]$} & [3] & {$[4]$} & [5] & {$[1]$} & {$[2]$} & {$[3]$} & {$[4]$} & [5] \\
\hline$\left(V_{t}\right)^{1 / 2}$ & $\begin{array}{l}0.013 \\
(0.003)\end{array}$ & - & - & - & - & $\begin{array}{l}0.074 \\
(0.006)\end{array}$ & - & - & - & - \\
\hline$V_{t}$ & - & $\begin{array}{l}0.013 \\
(0.003)\end{array}$ & - & - & $\begin{array}{l}0.014 \\
(0.003)\end{array}$ & - & $\begin{array}{l}0.083 \\
(0.004)\end{array}$ & - & - & $\begin{array}{l}0.096 \\
(0.016)\end{array}$ \\
\hline$S_{t}$ & - & - & $\begin{array}{c}-0.065 \\
(0.003)\end{array}$ & - & - & - & - & $\begin{array}{l}0.023 \\
(0.004)\end{array}$ & - & - \\
\hline$\left(S_{t}\right)^{1 / 3} \cdot\left(V_{t}\right)^{1 / 2}$ & - & - & - & $\begin{array}{c}-0.129 \\
(0.003)\end{array}$ & $\begin{array}{c}-0.138 \\
(0.003)\end{array}$ & - & - & - & $\begin{array}{c}-0.068 \\
(0.006)\end{array}$ & $\begin{array}{l}0.015 \\
(0.010)\end{array}$ \\
\hline
\end{tabular}

Notes -The table reports the models implied predictive regressions for equity excess returns. Panel A refers to the benchmark model in which shocks are skew-normally distributed. Panel B refers to the model with jumps in which the size is distributed as a negative exponential. All variables are standardized by subtracting their unconditional means and dividing by their standard deviations. The results were obtained by simulating the model at a monthly frequency. All standard errors are adjusted for heteroskedasticty.

the conditional mean of $x_{t}$ that is due to having time-variation in the conditional skewness. ${ }^{2}$ Equivalently, we shut down the relationship between skewness and future expected GDP growth rates. The results document that the performance of the model is much closer to the one featuring constant skewness. This result suggests that the superior performance of our benchmark model is mostly driven by the interaction between time-varying skewness and future expected growth rates. In the fifth column, we alter our benchmark specification by centering the skewness process around a negative value. The table documents that a negative average skewness enhances all the asset pricing predictions of the model. This means that we can interpret our benchmark model, in which skewness is on average equal to zero, as a conservative assessment of the asset pricing implications of time-varying skewness.

The performance of the model with jumps is featured in the last column of Table 4.2. It is apparent that our benchmark model and the Drechsler and Yaron (2011) model perform equally well when it comes to accounting for the unconditional asset pricing moments. The key difference between the two models lies in their predictions for the relationships between variance, skewness, and conditional risk premia. We explore this feature of the models in Table 4.3.

The table is divided into two parts. The first five columns (labeled "Benchmark Model") report several predictive regression of future excess returns onto lagged moments of the distribution of expected consumption growth. The next five columns (labeled "Model with Jumps") present the same set of regressions for the model with jumps.

\footnotetext{
${ }^{2}$ This is obtained by adding $-\varphi_{e} \sqrt{\sigma_{t}} \sqrt{2 / \pi} E_{t} \phi_{t+1}$ to the dynamics of $x_{t}$ in the system of equations in (4.1).
} 
The key result that emerges from Table 4.3 is that increasing economic uncertainty (as measured by $V_{t}$ ) is always associated with larger conditional equity risk premia. This statement applies both to our benchmark model with skew-normal innovations and to the model with jumps (see the first two rows of Table 4.3). The models, however, differ crucially when it comes to the response to skewness. In our model, rising skewness is always accompanied by lower future expected returns (see columns 3, 4, and 5 of the panel labeled "Benchmark Model"). This is because an increasing skewness of economic fundamentals is a telltale for better future expected growth rates of GDP. In the model with jumps, instead, skewness and volatility are negatively correlated. This means that the times in which skewness is the most negative are also the times in which uncertainty, as measured by variance, is the lowest. As a consequence future excess returns are a decreasing function of skewness. Furthermore, in the benchmark model with skew-normal innovations variance and third moment have independent predictive power for future returns (see specification in column 5 of Table 4.3). This is in sharp contrast with the model with jumps, in which the third moment becomes insignificant, after controlling for the variance of the expected growth rate.

\subsection{Sensitivity analysis}

Table 4.4 documents the sensitivity of our results to several alternative calibrations. Specifically, we consider three main specifications, in which we alter the degree of persistence of the predictive component, $\rho_{x}$, and the average volatility of the shocks, $\sqrt{\sigma_{c}}{ }^{3}$ We label the three cases as "Benchmark" $\left(\rho_{x}=0.962\right.$ and $\left.\sqrt{\sigma_{c}}=0.0068\right)$, "Medium Persistence" $\left(\rho_{x}=0.969\right.$ and $\left.\sqrt{\sigma_{c}}=0.0058\right)$, and "High Persistence" $\left(\rho_{x}=0.979\right.$ and $\left.\sqrt{\sigma_{c}}=0.0058\right)$. For each case, we report the results for a number of possible combinations of the parameters that govern the skewness dynamics $\left(\rho_{\nu}\right.$ ranging from 0.8 to 0.86 , and $\sqrt{\sigma_{\nu}}$ ranging between 0.2 and 0.6 ), as well as the calibration in which skewness is fixed at zero.

\footnotetext{
${ }^{3}$ We adjust $\sqrt{\sigma_{c}}$ in such a way that increasing the persistence parameter $\rho_{x}$ does not alter too much the overall volatility of consumption growth.
} 
The main messages looking at the three panels of Table 4.4 seem to be that Sharpe ratios increase on average by $50 \%$ thanks to the introduction of skewness dynamics and in some cases they can even get three times as large relative to the zero skewness specification. The volatility of consumption growth is usually moderately low, as the $95 \%$ confidence intervals from the simulations typically include the number estimated from actual data. For some of the most extreme calibrations Panel $\mathrm{C}$ documents that the autocorrelation of consumption growth becomes excessively large, but this is generally not an issue for the "Benchmark" and "Medium Persistence" calibrations. 


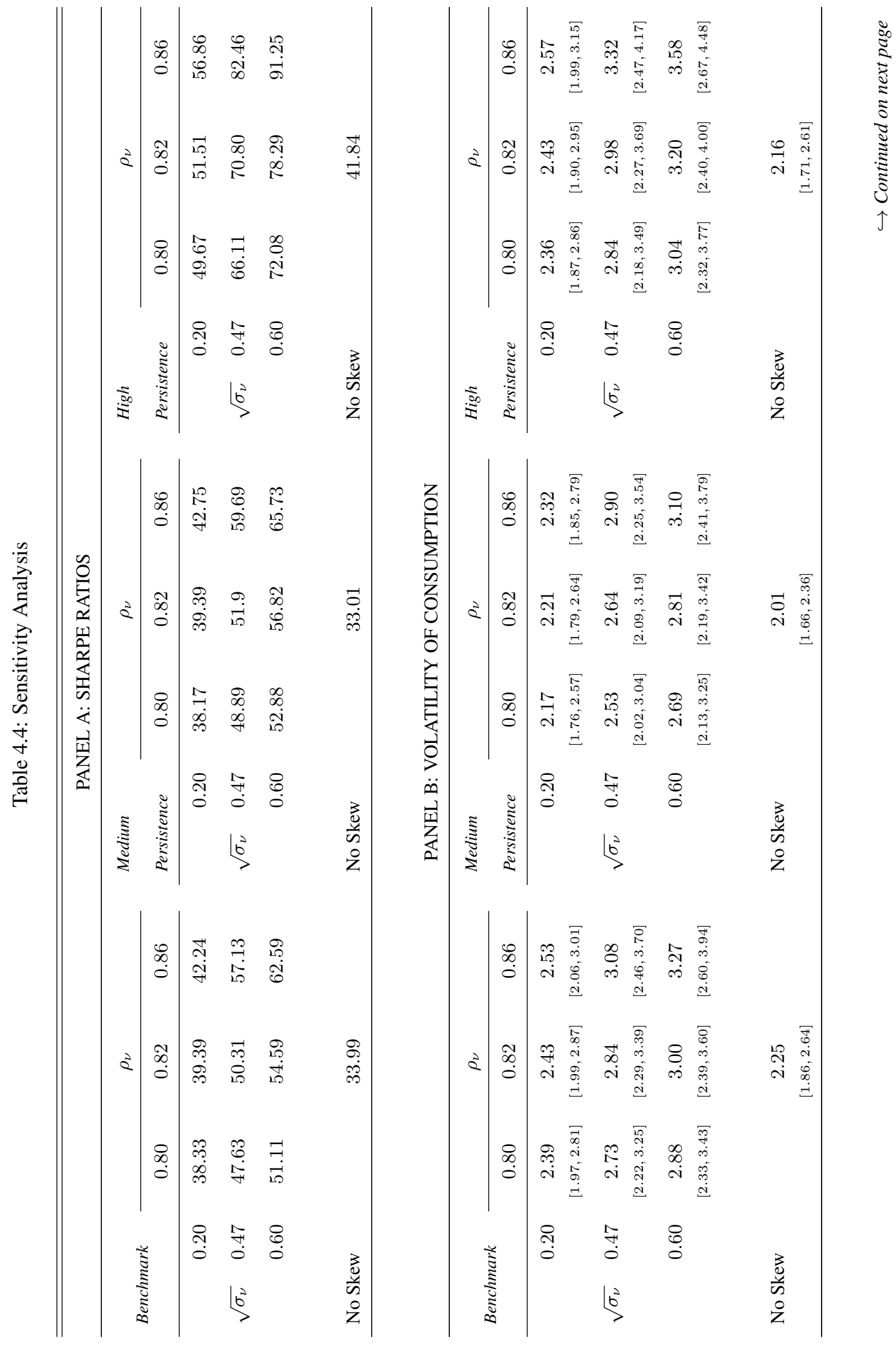




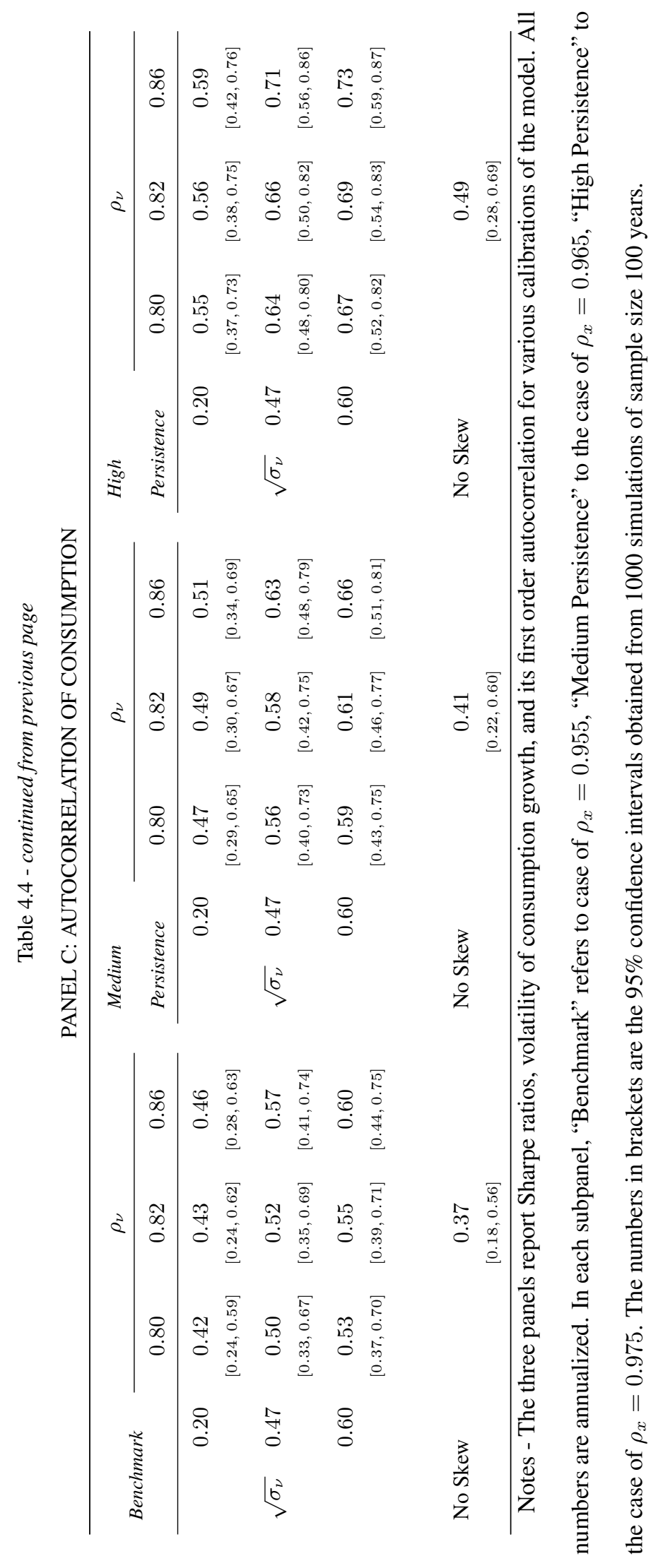




\section{CHAPTER 5: CONCLUSION}

Investors look at the predictions of future growth prospects made by professional forecasters. This dissertation documents that the entire distribution of such forecasts seems to matter as a larger cross sectional mean, a lower dispersion, and a larger degree of skewness predict lower equity excess returns going forward. The predictive ability of skewness is a novel empirical finding of this dissertation and it opens up the question of how to think about asymmetric growth prospects in the context of equilibrium asset pricing models. Introducing asymmetry in the distribution of expected consumption growth rates in a way that is consistent with the observed dynamics of consumption produces a sizeable increase in equity Sharpe ratios. Future developments in this literature should look at how these findings generalize to the cross-section of equity returns and to global equity markets. How to generate skewness in forecasts in the context of a production based asset pricing model would be an interesting avenue for future research as well. 


\section{APPENDIX A: Technical Appendix}

\section{A.1 The skew normal distribution}

General properties. A skew-normal distribution $S K N(\mu, \sigma, \nu)$ with location parameter $\mu$, scale parameter $\sigma$ and shape parameter $\nu$, has a probability density function:

$$
p(x)=\frac{1}{\sigma \pi} \exp \left\{-\frac{(x-\mu)^{2}}{2 \sigma^{2}}\right\} \int_{-\infty}^{\nu\left(\frac{x-\mu}{\sigma}\right)} \exp \left\{-\frac{t^{2}}{2}\right\} d t
$$

and closed form expressions fo the first three moments:

$$
\text { mean }=\mu+\sigma \phi \sqrt{\frac{2}{\pi}}, \text { variance }=\sigma^{2}\left(1-\frac{2 \phi^{2}}{\pi}\right), \text { skewness }=\frac{4-\pi}{2} \frac{(\phi \sqrt{2 / \pi})^{3}}{\left(1-2 \phi^{2} / \pi\right)^{3 / 2}},
$$

where $\phi=\frac{\nu}{\sqrt{1+\nu^{2}}}$.

Expectation of the exponent of a skew-normal random variable. Throughout the derivations of the analytical solutions of utility functions and price-dividend ratios, we shall often make use of the following lemma.

Lemma A.1.1. If $z \sim S K N(\mu, \sigma, \nu)$, then

$$
\begin{aligned}
\log E \exp \left\{\kappa_{1} z+\kappa_{2} z^{2}\right\}= & \log (2)-\frac{1}{2} \log \left(1-2 \kappa_{2} \sigma^{2}\right)+\frac{1}{2 \sigma^{2}}\left[\frac{\left(\kappa_{1} \sigma^{2}+\mu\right)^{2}}{1-2 \kappa_{2} \sigma^{2}}-\mu^{2}\right]+ \\
& \log \Phi\left(\frac{\nu}{\sigma^{2}} \cdot \frac{\kappa_{1} \sigma^{2}+\mu-\mu \sqrt{1-2 \kappa_{2} \sigma^{2}}}{\sqrt{1-2 \kappa_{2} \sigma^{2}+\nu^{2}}}\right)
\end{aligned}
$$

for any scalar $\kappa_{1}$ and $\kappa_{2}$, such that $1-2 \kappa_{2} \sigma^{2}>0$.

Proof. See Section A.9 of the appendix.

For the special case of $\kappa_{2}=0$ and $\sigma=1$, Lemma A.1.1 implies:

$$
\log E_{t} \exp \left\{\kappa_{1} z\right\}=\log (2)+\frac{\kappa_{1}\left(\kappa_{1}+2 \mu\right)}{2}+\log \Phi\left(\frac{\nu \kappa_{1}}{\sqrt{1+\nu^{2}}}\right)
$$

Lew's approximation. Lew (1981) provides the following approximation to the cumulative dis- 
tribution function of a standard normal distribution

$$
\Phi(z) \approx \frac{1}{2}+0.4 z, \text { for } \forall z \in[-0.5,0.5]
$$

and shows that the maximum absolute relative percentage error is $3 \%$. Note that this can also be written as:

$$
\Phi(z)=\frac{1}{2}+\frac{1}{2}\left[\sqrt{\frac{2}{\pi}}+\left(0.8-\sqrt{\frac{2}{\pi}}\right)\right] z=\frac{1}{2}+\frac{1}{2}\left[\sqrt{\frac{2}{\pi}}+\alpha\right] z
$$

where $\alpha=0.8-\sqrt{\frac{2}{\pi}}>0$.

Expectation of the exponent of a quadratic function of a normally distributed random variable. We shall use the following lemma to derive the analytical solution for the general case of our model.

Lemma A.1.2. Let $x \sim N(0,1)$ and let $A, B$, and $C$ be scalars. Then:

$$
\log E_{t} \exp \left\{A+B x+C x^{2}\right\}=\log \sqrt{\frac{1}{1-2 C}}+\frac{B^{2}}{2(1-2 C)}+A
$$

Proof. See Section A.9 of the appendix.

\section{A.2 Solution of the model}

Utility function. We shall start by expressing the utility function in a more convenient form. We define the value functions as the utility minus log-consumption:

$$
V_{t}=U_{t}-\log C_{t}=\delta \theta \log E_{t} \exp \left\{\frac{V_{t+1}+\Delta c_{t+1}}{\theta}\right\}
$$

where $\theta=1 /(1-\gamma)$. We shall decompose $V_{t}$ into the sum of two terms: one is linear in $x_{t}$, the other one is non-linear in $\sigma_{t}$ and $\nu_{t}$

$$
V_{t}=B x_{t}+\tilde{V}\left(\sigma_{t}, \nu_{t}\right)
$$


This separability is due to the assumption of unit intertemporal elasticity of substitution. We will use the notation $\widetilde{V}\left(\sigma_{t}, \nu_{t}\right)$ and $\widetilde{V}_{t}$ interchangeably. The value of $B$ is easy to obtain by plugging in the guess for $V_{t}$ and the process for $\Delta c_{t+1}$ in equation (A.1):

$$
\begin{aligned}
\widetilde{V}_{t}+B x_{t} & =\delta \theta \log E_{t} \exp \left\{\frac{\widetilde{V}_{t+1}+B x_{t+1}+\mu_{c}+x_{t}+\sqrt{\sigma_{t}^{c}} \varepsilon_{t+1}^{c}}{\theta}\right\} \\
& =\delta \theta \log E_{t} \exp \left\{\frac{\widetilde{V}_{t+1}+B \rho_{x} x_{t}+\varphi_{e} B \sqrt{\sigma_{t}^{x}} \varepsilon_{t+1}^{x}+\mu_{c}+x_{t}+\sqrt{\sigma_{t}^{c}} \varepsilon_{t+1}^{c}}{\theta}\right\} \\
& =\delta\left(B \rho_{x}+1\right) x_{t}+\delta \theta \log E_{t} \exp \left\{\frac{\widetilde{V}_{t+1}+\varphi_{e} B \sqrt{\sigma_{t}^{x}} \varepsilon_{t+1}^{x}+\mu_{c}+\sqrt{\sigma_{t}^{c}} \varepsilon_{t+1}^{c}}{\theta}\right\},
\end{aligned}
$$

from which it follows that

$$
B=\frac{\delta}{1-\delta \rho_{x}}
$$

The relevant recursion to solve for the remainder of the utility function then becomes

$$
\widetilde{V}_{t}=\delta \theta \log E_{t} \exp \left\{\frac{\mu_{c}+\varphi_{e} B \sqrt{\sigma_{t}^{x}} \varepsilon_{t+1}^{x}+\sqrt{\sigma_{t}^{c}} \varepsilon_{t+1}^{c}+\widetilde{V}_{t+1}}{\theta}\right\} .
$$

Stochastic discount factor. The stochastic discount factor is the intertemporal marginal rate of substitution:

$$
\begin{aligned}
m_{t+1} & =\log \left(\frac{\partial U_{t} / \partial C_{t+1}}{\partial U_{t} / \partial C_{t}}\right)=\log \delta-\Delta c_{t+1}+\frac{V_{t+1}+\Delta c_{t+1}}{\theta}-\log E_{t} \exp \left\{\frac{V_{t+1}+\Delta c_{t+1}}{\theta}\right\} \\
& =\log \delta-\left(1-\frac{1}{\theta}\right) \Delta c_{t+1}+\frac{1}{\theta}\left(\widetilde{V}_{t+1}-\frac{\widetilde{V}_{t}}{\delta}\right)+\frac{B}{\theta}\left(x_{t+1}-\frac{x_{t}}{\delta}\right) .
\end{aligned}
$$

Equity returns and risk-free rate. Given the stochastic discount factor in (A.3), the returns $R_{d, t}$ to a claim to any dividend process with log-growth rate $\Delta d_{t+1}$ can be computed by solving the Euler equation

$$
E_{t}\left[\exp \left\{m_{t+1}\right\} \frac{1+V_{d, t+1}}{V_{d, t}} \exp \left\{\Delta d_{t+1}\right\}\right]=1
$$

where $R_{d, t+1}=\frac{1+V_{d, t+1}}{V_{d, t}} \exp \left\{\Delta d_{t+1}\right\}$ and $V_{d, t}$ is the price-dividend ratio. The log-risk-free rate is computed as:

$$
r_{f, t}=-\log E_{t}\left[\exp \left\{m_{t+1}\right\}\right] .
$$




\section{A.3 Solution for the constant volatility case}

Assumptions. We assume that $\sigma_{t}^{c}=\sigma_{t}^{x}=\bar{\sigma}, \forall t$, and that $\phi_{t}=\frac{\nu_{t}}{\sqrt{1+\nu_{t}^{2}}}$ follows an $A R(1)$ process, that is

$$
\phi_{t+1}=\rho_{\phi} \phi_{t}+\sqrt{\sigma_{\phi}} \varepsilon_{t+1}^{\phi}, \text { where } \varepsilon_{t+1}^{\phi} \sim N(0,1) .
$$

Utility function. We shall solve for

$$
\widetilde{V}_{t}=\delta \theta \log E_{t} \exp \left\{\frac{\mu_{c}+\varphi_{e} B \sqrt{\bar{\sigma}} \varepsilon_{t+1}^{x}+\sqrt{\bar{\sigma}} \varepsilon_{t+1}^{c}+\widetilde{V}_{t+1}}{\theta}\right\} .
$$

In order to compute the conditional expectation on the right hand side of (A.4) we will make use of the properties of the skew-normal distribution that we have reported above. Specifically (from Lemma A.1.1), if $z$ follows a skew normal distribution $\operatorname{SKN}(0,1, \nu)$, then

$$
\log E_{t} \exp \left\{\kappa_{1} z\right\}=\log (2)+\frac{\kappa_{1}^{2}}{2}+\log \Phi\left(\frac{\nu \kappa_{1}}{\sqrt{1+\nu^{2}}}\right)
$$

Using Lew's approximation, we obtain $\log E_{t} \exp \left\{\kappa_{1} z\right\} \approx \frac{1}{2} \kappa_{1}^{2}+\log \left(1+0.8 \kappa_{1} \phi_{t}\right) \approx \frac{1}{2} \kappa_{1}^{2}+0.8 \kappa_{1} \phi_{t}$. Use the law of iterated expectations to compute

$$
\begin{aligned}
\widetilde{V}_{t} & =\delta \theta \log E_{t}\left[E\left[\exp \left\{\frac{\mu_{c}+\varphi_{e} B \sqrt{\bar{\sigma}} \varepsilon_{t+1}^{x}+\sqrt{\bar{\sigma}} \varepsilon_{t+1}^{c}+\widetilde{V}_{t+1}}{\theta}\right\} \mid\left(\varepsilon_{t+1}^{c}, \varepsilon_{t+1}^{\phi}\right)\right]\right] \\
& =\delta \theta \log E_{t} \exp \left\{\frac{\mu_{c}+\log \left(1+\Phi\left(\varphi_{e} B \sqrt{\bar{\sigma}} \phi_{t+1}\right)\right)+\sqrt{\bar{\sigma}} \varepsilon_{t+1}^{c}+\widetilde{V}_{t+1}}{\theta}\right\} \\
& =\delta \theta \log E_{t} \exp \left\{\left[\mu_{c}+\frac{\varphi_{e}^{2} B^{2} \bar{\sigma}}{2 \theta}+0.8 \varphi_{e} B \sqrt{\bar{\sigma}} \phi_{t+1}+\sqrt{\bar{\sigma}} \varepsilon_{t+1}^{c}+\widetilde{V}_{t+1}\right] \cdot \frac{1}{\theta}\right\}
\end{aligned}
$$

We guess that the solution of this value function is linear in $\phi_{t}: \widetilde{V}_{t}=V_{0}+V_{\phi} \phi_{t}$. By substituting our guess into the value function above, we get:

$\widetilde{V}_{t}=\delta\left[\mu_{c}+\frac{\varphi_{e}^{2} B^{2} \bar{\sigma}}{2 \theta}+V_{0}+\frac{\bar{\sigma}}{2 \theta}+\frac{1}{2 \theta}\left(0.8 \varphi_{e} B \sqrt{\bar{\sigma}}+V_{\phi}\right)^{2} \sigma_{\phi}\right]+\phi_{t} \cdot \delta\left[0.8 \varphi_{e} B \sqrt{\bar{\sigma}} \rho_{\phi}+V_{\phi} \rho_{\phi}\right]$. 
Matching coefficients:

$$
V_{0}=\frac{\delta}{1-\delta}\left[\mu_{c}+\frac{\bar{\sigma}}{2 \theta}+\frac{\varphi_{e}^{2} B^{2} \bar{\sigma}}{2 \theta}+\frac{1}{2 \theta}\left(0.8 \varphi_{e} B \sqrt{\bar{\sigma}}+V_{\phi}\right)^{2} \sigma_{\phi}\right], \text { and } V_{\phi}=\frac{0.8 \delta \rho_{\phi} \varphi_{e} B \sqrt{\bar{\sigma}}}{1-\delta \rho_{\phi}}
$$

Stochastic discount factor. The stochastic discount factor is obtained as the marginal rate of intertemporal substitution:

$$
\begin{aligned}
m_{t+1}= & \log \delta-\left(1-\frac{1}{\theta}\right) \Delta c_{t+1}+\frac{V_{0}}{\theta}+\frac{V_{\phi}}{\theta} \phi_{t+1}+\frac{B}{\theta} x_{t+1}- \\
& \log E_{t} \exp \left\{\frac{V_{0}+V_{\phi} \phi_{t+1}+B x_{t+1}+\mu_{c}+x_{t}+\sqrt{\bar{\sigma}} \varepsilon_{t+1}^{c}}{\theta}\right\} \\
= & \log \delta-\mu_{c}-x_{t}-\left(1-\frac{1}{\theta}\right) \sqrt{\sigma^{c}} \varepsilon_{t+1}^{c}+\frac{V_{\phi}}{\theta} \sqrt{\sigma_{\phi}} \varepsilon_{t+1}^{\phi}+\frac{B}{\theta} \varphi_{e} \sqrt{\bar{\sigma}} \varepsilon_{t+1}^{x}- \\
& \log E_{t} E\left[\exp \left\{\left[V_{\phi} \sqrt{\sigma_{\phi}} \varepsilon_{t+1}^{\phi}+B \varphi_{e} \sqrt{\bar{\sigma}} \varepsilon_{t+1}^{x}+\sqrt{\bar{\sigma}} \varepsilon_{t+1}^{c}\right] \frac{1}{\theta}\right\} \mid \varepsilon_{t+1}^{\phi}\right]
\end{aligned}
$$

Using Lemma A.1.1 and Lew's approximation, we can solve equation (A.6) and obtain:

$$
\begin{aligned}
m_{t+1}= & m_{0}-x_{t}-\frac{0.8 B \varphi_{e} \sqrt{\bar{\sigma}} \rho_{\phi}}{\theta} \phi_{t}-\left(1-\frac{1}{\theta}\right) \sqrt{\bar{\sigma}} \varepsilon_{t+1}^{c}+\frac{V_{\phi} \sqrt{\sigma_{\phi}}}{\theta} \varepsilon_{t+1}^{\phi}+ \\
& \frac{B \varphi_{e} \sqrt{\bar{\sigma}}}{\theta} \varepsilon_{t+1}^{x}
\end{aligned}
$$

where $m_{0}=\log \delta-\mu_{c}-\frac{\bar{\sigma}}{2 \theta^{2}}-\frac{B^{2} \varphi_{e}^{2} \bar{\sigma}}{2 \theta^{2}}-\frac{1}{2 \theta^{2}}\left(\frac{0.8 B \varphi_{e}}{1-\delta \rho_{\phi}}\right)^{2} \bar{\sigma} \sigma_{\phi}$. It follows that

$$
m_{t+1}-E_{t} m_{t+1}=-\left(1-\frac{1}{\theta}\right) \sqrt{\bar{\sigma}} \varepsilon_{t+1}^{c}+\frac{V_{\phi} \sqrt{\sigma_{\phi}}}{\theta} \varepsilon_{t+1}^{\phi}+\frac{B \varphi_{e} \sqrt{\bar{\sigma}}}{\theta} \varepsilon_{t+1}^{x}
$$

where

$$
B=\frac{\delta}{1-\delta \rho_{x}}, \quad V_{\phi}=\frac{0.8 \varphi_{e} B \delta \rho_{\phi}}{1-\delta \rho_{\phi}} \sqrt{\bar{\sigma}}, \quad \theta=\frac{1}{1-\gamma}
$$

Equity returns. We compute returns on a claim to the following dividend process:

$$
\Delta d_{t}=\lambda_{1} \mu_{c}+\lambda\left(x_{t-1}+\sqrt{\bar{\sigma}} \varepsilon_{t}^{c}\right)+\sqrt{\sigma_{d}} \sqrt{\bar{\sigma}} \varepsilon_{t}^{d}, \quad \varepsilon_{t}^{d} \sim N(0,1)
$$


The Euler equation is:

$$
E_{t} \exp \left\{m_{t+1}+\lambda_{1} \mu_{c}+\lambda\left(x_{t}+\sqrt{\bar{\sigma}} \varepsilon_{t+1}^{c}\right)+\sqrt{\sigma_{d}} \sqrt{\bar{\sigma}} \varepsilon_{t+1}^{d}\right\}\left(1+\exp \left\{v_{d, t+1}\right\}\right)=\exp \left\{v_{d, t}\right\}
$$

where $v_{d, t}=\log \left(V_{d, t}\right)$. Take a linear expansion of $\log \left(1+\exp \left\{v_{d, t+1}\right\}\right)$ about the unconditional mean of $v_{d, t}$, denoted as $\bar{v}$ :

$$
\log \left(1+\exp \left\{v_{d, t+1}\right\}\right)=K_{0}+K_{1} v_{d, t+1},
$$

where

$$
K_{1}=\frac{\exp \{\bar{v}\}}{1+\exp \{\bar{v}\}} \text { and } K_{0}=\log (1+\exp \{\bar{v}\})-K_{1} \bar{v}
$$

Substituting this into the Euler equation and taking logs of both sides yields:

$$
v_{d, t}=\log E_{t} \exp \left\{m_{t+1}+\lambda_{1} \mu_{c}+\lambda\left(x_{t}+\sqrt{\bar{\sigma}} \varepsilon_{t+1}^{c}\right)+\sqrt{\sigma_{d}} \sqrt{\bar{\sigma}} \varepsilon_{t+1}^{d}+K_{0}+K_{1} v_{d, t+1}\right\}
$$

Guess that $v_{d, t}=v_{0}+v_{x} x_{t}+v_{\phi} \phi_{t}$. Then:

$$
\begin{aligned}
v_{d, t}= & \log E_{t} \exp \left\{m_{0}-x_{t}-m_{\phi} \phi_{t}-\sqrt{z_{c}} \varepsilon_{t+1}^{c}-\sqrt{z_{\phi}} \varepsilon_{t+1}^{\phi}-\sqrt{z_{x}} \varepsilon_{t+1}^{x}+\right. \\
& \left.+\lambda_{1} \mu_{c}+\lambda\left(x_{t}+\sqrt{\bar{\sigma}} \varepsilon_{t+1}^{c}\right)+\sqrt{\sigma_{d}} \sqrt{\bar{\sigma}} \varepsilon_{t+1}^{d}+K_{0}+K_{1}\left(v_{0}+v_{x} x_{t+1}+v_{\phi} \phi_{t+1}\right)\right\}
\end{aligned}
$$

where

$$
m_{\phi}=\frac{0.8 B \varphi_{e} \sqrt{\bar{\sigma}} \rho_{\phi}}{\theta}, \quad \sqrt{z_{c}}=\left(1-\frac{1}{\theta}\right) \sqrt{\bar{\sigma}}, \quad \sqrt{z_{\phi}}=-\frac{V_{\phi} \sqrt{\sigma_{\phi}}}{\theta}, \quad \sqrt{z_{x}}=-\frac{B \varphi_{e} \sqrt{\bar{\sigma}}}{\theta} .
$$

Using the law of iterated expectations, we get

$$
\begin{aligned}
v_{d, t}= & m_{0}+\lambda_{1} \mu_{c}+K_{0}+K_{1} v_{0}+\frac{\left(\sqrt{z_{c}}-\lambda \sqrt{\bar{\sigma}}\right)^{2}}{2}+\frac{\sigma_{d} \bar{\sigma}}{2}+\frac{1}{2}\left(\sqrt{z_{x}}-K_{1} v_{x} \varphi_{e} \sqrt{\bar{\sigma}}\right)^{2} \\
& +\frac{1}{2}\left[\left(\sqrt{z_{\phi}}-K_{1} v_{\phi} \sqrt{\sigma_{\phi}}\right)+0.8\left(\sqrt{z_{x}}-K_{1} v_{x} \varphi_{e} \sqrt{\bar{\sigma}}\right) \sqrt{\sigma_{\phi}}\right]^{2}+\left[K_{1} v_{x} \rho_{x}-(1-\lambda)\right] x_{t} \\
& +\left[K_{1} v_{\phi} \rho_{\phi}-m_{\phi}-0.8\left(\sqrt{z_{x}}-K_{1} v_{x} \varphi_{e} \sqrt{\bar{\sigma}}\right) \rho_{\phi}\right] \phi_{t}
\end{aligned}
$$


Matching Coefficients:

$$
\begin{aligned}
v_{0}= & \frac{1}{1-K_{1}}\left\{m_{0}+\lambda_{1} \mu_{c}+K_{0}+\frac{\left(\sqrt{z_{c}}-\lambda \sqrt{\bar{\sigma}}\right)^{2}}{2}+\frac{\sigma_{d} \bar{\sigma}}{2}+\frac{1}{2}\left(\sqrt{z_{x}}-K_{1} v_{x} \varphi_{e} \sqrt{\bar{\sigma}}\right)^{2}\right. \\
& \left.+\frac{1}{2}\left[\left(\sqrt{z_{\phi}}-K_{1} v_{\phi} \sqrt{\sigma_{\phi}}\right)+0.8\left(\sqrt{z_{x}}-K_{1} v_{x} \varphi_{e} \sqrt{\bar{\sigma}}\right) \sqrt{\sigma_{\phi}}\right]^{2}\right\} \\
v_{x}= & \frac{\lambda-1}{1-K_{1} \rho_{x}} \\
v_{\phi}= & \frac{0.8 K_{1}(\lambda-1)}{\left(1-K_{1} \rho_{\phi}\right)\left(1-K_{1} \rho_{x}\right)} \varphi_{e} \rho_{\phi} \sqrt{\bar{\sigma}} .
\end{aligned}
$$

Therefore the log-equity returns are equal to

$$
\begin{aligned}
\log R_{t+1}= & \bar{r}+x_{t}+\underbrace{v_{\phi}\left(K_{1} \rho_{\phi}-1\right)}_{<0} \phi_{t}+K_{1} v_{\phi} \sqrt{\sigma_{\phi}} \varepsilon_{t+1}^{\phi}+K_{1} v_{x} \varphi_{e} \sqrt{\bar{\sigma}} \varepsilon_{t+1}^{x}+\lambda \sqrt{\bar{\sigma}} \varepsilon_{t+1}^{c}+ \\
& \sqrt{\sigma_{d}} \sqrt{\bar{\sigma}} \varepsilon_{t+1}^{d}
\end{aligned}
$$

where $\bar{r}=K_{0}+K_{1} v_{0}-v_{0}+\lambda_{1} \mu_{c}$.

Risk-free rate. The risk-free rate is obtained as

$$
r_{f}=-\log E_{t} \exp \left\{m_{t+1}\right\}=-\log \delta+\mu_{c}+\left(\frac{1}{\theta}-\frac{1}{2}\right) \bar{\sigma}+x_{t}
$$

Conditional Equity Returns. Using the calculations above, we obtain the following expression for the returns of equity in excess of the risk-free asset:

$$
\begin{aligned}
\log R_{t+1}-r_{f, t}= & \bar{r}^{e}+\left(K_{1} v_{\phi} \rho_{\phi}-v_{\phi}\right) \phi_{t}+v_{\phi} \sqrt{\sigma_{\phi}} \varepsilon_{t+1}^{\phi}+\lambda \sqrt{\bar{\sigma}} \varepsilon_{t+1}^{c}+\sqrt{\sigma_{d}} \varepsilon_{t+1}^{d}+ \\
& K_{1} v_{x} \varphi_{e} \sqrt{\bar{\sigma}} \varepsilon_{t+1}^{x}
\end{aligned}
$$

where $\bar{r}^{e}=\log (1+\exp \{\bar{v}\})-\bar{v}+\log (\delta)-\left(\frac{1}{\theta}-\frac{1}{2}\right) \bar{\sigma}$. Since $E_{t}\left[\varepsilon_{t+1}^{x}\right]=\rho_{\phi} \sqrt{2 / \pi} \phi_{t}$, we get that the conditional expectation of the excess return is

$$
\begin{aligned}
E_{t}\left[\log R_{t+1}-r_{f, t}\right] & =\bar{r}^{e}+\left(K_{1} \rho_{\phi}-1\right) v_{\phi} \phi_{t}+K_{1} v_{x} \varphi_{e} \sqrt{\bar{\sigma}} \rho_{\phi} \sqrt{2 / \pi} \phi_{t} \\
& =\bar{r}^{e}-(\sqrt{2 / \pi}+\alpha) \frac{K_{1}(\lambda-1)}{1-K_{1} \rho_{x}} \varphi_{e} \sqrt{\bar{\sigma}} \rho_{\phi} \cdot \phi_{t}+\sqrt{2 / \pi} \frac{K_{1}(\lambda-1)}{1-K_{1} \rho_{x}} \varphi_{e} \sqrt{\bar{\sigma}} \rho_{\phi} \cdot \phi_{t}
\end{aligned}
$$




$$
=\bar{r}^{e}-\alpha \frac{K_{1}(\lambda-1)}{1-K_{1} \rho_{x}} \varphi_{e} \sqrt{\bar{\sigma}} \rho_{\phi} \cdot \phi_{t}
$$

where $\alpha=0.8-\sqrt{2 / \pi}>0$ and it corresponds to the coefficient of approximation of the normal cumulative distribution function in the Lew (1981) approximation.

Alternative approximations. In section A.14.1 of the appendix, we document that the results reported in this section are robust to several alternative approximations.

\section{A.4 Solution of the model with time-varying volatility and skewness}

We assume that the variance evolves according to the following $\mathrm{AR}(1)$ process

$$
\sigma_{t}^{x}=\sigma_{t}^{c}=\sigma_{t}=\bar{\sigma}\left(1-\rho_{\sigma}\right)+\rho_{\sigma} \sigma_{t-1}+\sqrt{\sigma_{\varepsilon}} \varepsilon_{t}^{\sigma}
$$

In the interest of space, we report only in this section the main steps needed to obtain the solution for equity returns associated with this version of the model. All the details of the solution as well as the associated coefficients are reported in section A.11 of the appendix.

We show that the solution of the utility function is linear in $\phi_{t}, \phi_{t}^{2}, \sqrt{\sigma_{t}}, \sigma_{t}, \phi_{t} \sqrt{\sigma_{t}}$

$$
\widetilde{V}_{t}=V_{0}+V_{\phi, 1} \phi_{t}+V_{\phi, 2} \phi_{t}^{2}+V_{\sigma, 1} \sqrt{\sigma_{t}}+V_{\sigma, 2} \sigma_{t}+V_{\phi, \sigma} \phi_{t} \sqrt{\sigma_{t}}
$$

where the coefficients $V_{0}, V_{\phi, 1}, V_{\phi, 2}, V_{\sigma, 1}, V_{\sigma, 2}$, and $V_{\phi, \sigma}$ are reported in equation (A.17).

We show in section A.11.2 of the appendix that the innovations to the stochastic discount factor are

$$
\begin{aligned}
m_{t+1}-E_{t} m_{t+1}= & -\left(1-\frac{1}{\theta}\right) \sigma_{t} \varepsilon_{t+1}^{c}+ \\
& \frac{1}{\theta} B \varphi_{e} \sqrt{\sigma_{t}} \varepsilon_{t+1}^{x}+\frac{1}{\theta}\left(V_{\sigma, 1} \frac{1}{2 \sqrt{\bar{\sigma}}}+V_{\sigma, 2}+V_{\phi, \sigma} \rho_{\phi} \frac{1}{2 \sqrt{\bar{\sigma}}} \phi_{t}\right) \cdot \varepsilon_{t+1}^{\sigma} \\
& +\frac{1}{\theta}\left[V_{\phi, 1}+2 V_{\phi, 2} \rho_{\phi} \phi_{t}+V_{\phi, \sigma} \sqrt{\bar{\sigma}}\left(1-\rho_{\sigma}\right)+\rho_{\sigma} \sqrt{\sigma_{t}} V_{\phi, \sigma}\right] \sqrt{\sigma_{\phi}} \varepsilon_{t+1}^{\phi} \\
& +\frac{1}{\theta} V_{\phi, \sigma} \frac{\sqrt{\sigma_{\varepsilon}}}{2 \sqrt{\bar{\sigma}}} \sqrt{\sigma_{\phi}} \varepsilon_{t+1}^{\sigma} \varepsilon_{t+1}^{\phi}+V_{\phi, 2} \sigma_{\phi}\left(\varepsilon_{t+1}^{\phi}\right)^{2} .
\end{aligned}
$$


The risk-free rate is derived in section A.11.4 and it is equal to:

$$
r_{f}=-\log E_{t} \exp \left\{m_{t+1}\right\}=-\log \delta+\mu_{c}+\left(\frac{1}{\theta}-\frac{1}{2}\right) \sigma_{t}+x_{t}
$$

We then document that using the Euler equation and the Campbell and Shiller (1988) approximation, we can express the logarithm of the price-dividend ratio of a claim to the dividend process

$$
\Delta d_{t}=\lambda_{1} \mu_{c}+\lambda\left(x_{t-1}+\sqrt{\sigma_{t-1}^{c}} \varepsilon_{t}^{c}\right)+\sqrt{\sigma_{d}} \sqrt{\sigma_{t-1}^{c}} \varepsilon_{t}^{d}
$$

as $v_{d, t}=v_{0}+v_{x, 1} x_{t}+v_{\phi, 1} \phi_{t}+v_{\phi, 2} \phi_{t}^{2}+v_{\sigma, 1} \sqrt{\sigma_{t}}+v_{\sigma, 2} \sigma_{t}+v_{\phi, \sigma} \phi_{t} \sqrt{\sigma_{t}}$, where the coefficients $v_{0}, v_{x, 1}, v_{\phi, 1}, v_{\phi, 2}, v_{\sigma, 1}, v_{\sigma, 2}$, and $v_{\phi, \sigma}$ are reported in the system of equations (A.18). This allows us to derive equity returns and the associated conditional equity risk premia (see section A.11.5 of the appendix) as $\log R_{t+1}=\log \left(1+\exp \left\{v_{d, t+1}\right\}\right)-v_{d, t}+\Delta d_{t+1}$.

\section{A.5 GMM estimation}

Setup. The transition dynamics of the three state variables $\left(x_{t}, \sigma_{t}, \phi_{t}\right)$ is defined as follows:

$$
\begin{aligned}
x_{t} & =\mu_{x}\left(1-\rho_{x}\right)+\rho_{x} x_{t-1}+\varphi_{e} \sqrt{\sigma_{t-1}} \varepsilon_{t}^{x} \\
\sigma_{t} & =\bar{\sigma}\left(1-\rho_{\sigma}\right)+\rho_{\sigma} \sigma_{t-1}+\sqrt{\sigma_{\varepsilon}} \varepsilon_{t}^{\sigma} \\
\phi_{t} & =\bar{\phi}\left(1-\rho_{\phi}\right)+\rho_{\phi} \phi_{t-1}+\sqrt{\sigma_{\phi}} \varepsilon_{t}^{\phi}
\end{aligned}
$$

where $\varepsilon_{t}^{x} \sim S K N\left(0,1, \nu_{t}\right)$ with $\nu_{t}=\phi_{t} / \sqrt{1-\phi_{t}^{2}}$ and $\varepsilon_{t}^{\sigma}, \varepsilon_{t}^{\phi}$ are distributed as standard Normals.

At each date $t$, we construct the cross sectional moments of the distribution of expected growth rates as:

$$
\begin{aligned}
\widehat{E}_{t}^{c s}\left(\Delta c_{t+1}\right) & =\frac{1}{n} \sum_{i=1}^{n} E_{t}^{i}\left(\Delta c_{t+1}\right) \\
\widehat{V}_{t}^{c s}\left(\Delta c_{t+1}\right) & =\frac{1}{n} \sum_{i=1}^{n}\left[E_{t}^{i}\left(\Delta c_{t+1}\right)-\widehat{E}_{t}^{c s}\left(\Delta c_{t+1}\right)\right]^{2}
\end{aligned}
$$




$$
\widehat{S}_{t}^{c s}\left(\Delta c_{t+1}\right)=\frac{\frac{1}{n} \sum_{i=1}^{n}\left[E_{t}^{i}\left(\Delta c_{t+1}\right)-\widehat{E}_{t}^{c s}\left(\Delta c_{t+1}\right)\right]^{3}}{\left(\widehat{V}_{t}^{c s}\left(\Delta c_{t+1}\right)\right)^{3 / 2}}
$$

Based on $\widehat{E}_{t}^{c s}, \widehat{V}_{t}^{c s}$, and $\widehat{S}_{t}^{c s}$, we obtain the time series for $\widehat{x}_{t}, \widehat{\sigma}_{t}$, and $\widehat{\phi}_{t}$ as follows. Let $\widehat{A}_{t}^{c s}=$ $\left(\widehat{S}_{t}^{c s}\right)^{1 / 3}$, and note that in population:

$$
A_{t}^{c s}=\left[\frac{E_{t}\left(x_{t+1}-E_{t}\left(x_{t+1}\right)\right)^{3}}{V_{t}\left(x_{t+1}^{3 / 2}\right)}\right]^{1 / 3}=\left(\frac{4-\pi}{2}\right)^{1 / 3} \sqrt{\frac{2}{\pi}} \frac{E_{t} \phi_{t+1}}{\left[1-2\left(E_{t} \phi_{t+1}\right)^{2} / \pi\right]^{1 / 2}} .
$$

Rearranging the above equation and defining $\widehat{\phi}_{t}$ as an estimate of $E_{t}\left[\phi_{t}\right]$, we obtain that:

$$
\widehat{\phi}_{t}=\frac{\widehat{A}_{t}^{c s}}{\sqrt{K_{\phi}^{2}+2\left(\widehat{A}_{t}^{c s}\right)^{2} / \pi}}
$$

where $K_{\phi}=\left(\frac{4-\pi}{2}\right)^{1 / 3} \sqrt{\frac{2}{\pi}}$. Given the AR(1) transition dynamics of $\phi_{t}, \widehat{\phi}_{t}$ evolves according to

$$
\widehat{\phi}_{t}=\bar{\phi}\left(1-\rho_{\phi}\right)+\rho_{\phi} \widehat{\phi}_{t-1}+\rho_{\phi} \sqrt{\sigma_{\phi}} \varepsilon_{t}^{\phi} .
$$

Using the properties of the skew-normal distribution, we obtain that:

$$
V_{t}\left(x_{t+1}\right)=\varphi_{e} \sigma_{t}\left(1-\frac{2}{\pi} \widehat{\phi}_{t}^{2}\right)
$$

This allows us to retrieve the variance process $\widehat{\sigma}_{t}$ as

$$
\widehat{\sigma}_{t}=\widehat{V}_{t}^{c s}\left(1-\frac{2}{\pi} \widehat{\phi}_{t}^{2}\right)^{-1}
$$

Finally, we set

$$
\widehat{x}_{t}=\widehat{E}_{t}^{c s} .
$$


Estimation. The estimation strategy consists in matching moments of $x_{t}, \sigma_{t}$, and $\phi_{t}$ in (A.11) with the empirical counterparts based on $\widehat{x}_{t}, \widehat{\sigma}_{t}$, and $\widehat{\phi}_{t}$ in equations (A.14), (A.13), and (A.12). We estimate 8 parameters $\left(\rho_{x}, \mu_{x}, \bar{\sigma}, \rho_{\sigma}, \sigma_{\varepsilon}, \rho_{\phi}, \bar{\phi}, \sigma_{\phi}\right)$, using the following 8 moment conditions:

1. $E\left(\widehat{\phi}_{t}\right)=\frac{1}{T} \sum_{t=1}^{T} \widehat{\phi}_{t}=\bar{\phi}$

2. $V\left(\widehat{\phi}_{t}\right)=\frac{1}{T} \sum_{t=1}^{T}\left(\widehat{\phi}_{t}-\bar{\phi}\right)^{2}=\frac{\rho_{\phi}^{2} \sigma_{\phi}}{1-\rho_{\phi}^{2}}$

3. $E\left[\left(\widehat{\phi}_{t}-\bar{\phi}\right)\left(\widehat{\phi}_{t-1}-\bar{\phi}\right)\right]=\frac{1}{T-1} \sum_{t=2}^{T}\left(\widehat{\phi}_{t}-\bar{\phi}\right)\left(\widehat{\phi}_{t-1}-\bar{\phi}\right)=\frac{\rho_{\phi}^{3} \sigma_{\phi}}{1-\rho_{\phi}^{2}}$

4. $E\left(\widehat{\sigma}_{t}\right)=\frac{1}{T} \sum_{t=1}^{T} \widehat{\sigma}_{t}=\bar{\sigma}$

5. $V\left(\sigma_{t}\right)=\frac{1}{T} \sum_{t=1}^{T}\left(\widehat{\sigma}_{t}-\bar{\sigma}\right)^{2}=\frac{\sigma_{\varepsilon}}{1-\rho_{\sigma}^{2}}$

6. $E\left[\left(\widehat{\sigma}_{t}-\bar{\sigma}\right)\left(\widehat{\sigma}_{t-1}-\bar{\sigma}\right)\right]=\frac{1}{T-1} \sum_{t=2}^{T}\left(\widehat{\sigma}_{t}-\bar{\sigma}\right)\left(\widehat{\sigma}_{t-1}-\bar{\sigma}\right)=\frac{\rho_{\sigma} \sigma_{\varepsilon}}{1-\rho_{\sigma}^{2}}$

7. $E\left(\widehat{x}_{t}\right)=\mu_{x}+\frac{\varphi_{e} \sqrt{\bar{\sigma}} \sqrt{2 / \pi} \bar{\phi}}{1-\rho_{x}}=\frac{1}{T} \sum_{t=1}^{T} \widehat{E}_{t}^{c s}$

8. $E\left(\widehat{x}_{t}-\mu_{x}-\varphi_{e} \sqrt{\widehat{\sigma}_{t-1}} \sqrt{\frac{2}{\pi}} \widehat{\phi}_{t-1}\right) \cdot\left(\widehat{x}_{t-1}-\mu_{x}\right)=\frac{1}{T-1} \sum_{t=2}^{T}\left(\widehat{x}_{t}-\mu_{x}-\varphi_{e} \sqrt{\widehat{\sigma}_{t-1}} \sqrt{\frac{2}{\pi}} \widehat{\phi}_{t-1}\right)\left(\widehat{x}_{t-1}-\right.$ $\left.\mu_{x}\right)=\frac{\rho_{x}}{T-1} \sum_{t=2}^{T}\left(\widehat{E}_{t}^{c s}-\mu_{x}\right)^{2}$.

We report the details of the derivation of the last two moments conditions in Section A.12 of the appendix. Last, we set the parameter $\varphi_{e}$ to match the first order autocorrelation of consumption growth at semi-annual frequency. We show in section A.12 of the appendix that:

$$
A C_{1}\left(\Delta c_{t}\right)=\frac{\varphi_{e}^{2} K_{N}}{1-\rho_{x}^{2}+\varphi_{e}^{2} K_{D}}
$$

where

$$
\begin{aligned}
K_{N} & =\rho_{x}+\frac{2}{\pi} \frac{\rho_{\phi}^{2} \sigma_{\phi}}{\left(1-\rho_{\phi}^{2}\right)\left(1-\rho_{x} \rho_{\phi}\right)} \cdot\left(\rho_{x}+\rho_{\phi}\right) \\
K_{D} & =1+\frac{2}{\pi} \frac{\rho_{\phi}^{2} \sigma_{\phi}}{\left(1-\rho_{\phi}^{2}\right)\left(1-\rho_{x} \rho_{\phi}\right)} \cdot\left(1+\rho_{x} \rho_{\phi}\right) .
\end{aligned}
$$




\section{A.6 Conditional moments in the model with jumps}

In this section we compute the first three conditional moments of

$$
x_{t+1}=\rho_{x} x_{t}+\varphi_{e} \sqrt{\sigma_{t}} \varepsilon_{t+1}^{x}+J_{t+1}^{x}
$$

where

$$
\varepsilon_{t+1}^{x} \sim N(0,1), \quad J_{t+1}^{x}=\sum_{j=1}^{N_{t+1}^{x}} \xi_{j, t+1}^{x}, \quad N_{t+1}^{x} \sim \operatorname{Poisson}\left(l_{0}^{x}+l_{1}^{x} \sigma_{t}\right), \quad \xi_{t+1}^{x} \sim-\operatorname{Exp}\left(\mu_{x}\right)+\mu_{x} .
$$

The first three conditional moments of $\varphi_{e} \sqrt{\sigma_{t}} \varepsilon_{t+1}^{x}$ are

$$
E_{t}\left[\varphi_{e} \sqrt{\sigma_{t}} \varepsilon_{t+1}^{x}\right]=0, \quad V_{t}\left[\varphi_{e} \sqrt{\sigma_{t}} \varepsilon_{t+1}^{x}\right]=\varphi_{e}^{2} \sigma_{t}, \quad E_{t}\left[\left(\varphi_{e} \sqrt{\sigma_{t}} \varepsilon_{t+1}^{x}\right)^{3}\right]=0 .
$$

The conditional mean of $J_{t+1}^{x}$ is

$$
E_{t}\left[J_{t+1}^{x}\right]=E_{t}\left[\sum_{j=1}^{N_{t+1}^{x}} \xi_{j, t+1}^{x}\right]=E_{t}\left[E\left(\sum_{j=1}^{N_{t+1}^{x}} \xi_{j, t+1}^{x} \mid N_{t+1}^{x}\right)\right]=E_{t}\left[N_{t+1}^{x} \cdot 0\right]=0 .
$$

The conditional variance of $J_{t+1}^{x}$ is

$$
\begin{aligned}
V_{t}\left[J_{t+1}^{x}\right] & =E_{t}\left[\left(J_{t+1}^{x}\right)^{2}\right]=E_{t}\left[E\left(\left(\sum_{j=1}^{N_{t+1}^{x}} \xi_{j, t+1}^{x}\right)^{2} \mid N_{t+1}^{x}\right)\right]=E_{t}\left[N_{t+1}^{x} \cdot V_{t}\left(\xi_{j, t+1}^{x}\right)\right] \\
& =E_{t}\left[N_{t+1}^{x} \cdot \mu_{x}^{2}\right] \\
& =\left(l_{0}^{x}+l_{1}^{x} \sigma_{t}\right) \mu_{x}^{2} .
\end{aligned}
$$

The conditional third central moment of $J_{t+1}^{x}$ is computed as:

$$
E_{t}\left[\left(J_{t+1}^{x}\right)^{3}\right]=E_{t}\left[E\left(\left(\sum_{j=1}^{N_{t+1}^{x}} \xi_{j, t+1}^{x}\right)^{3} \mid N_{t+1}^{x}\right)\right]
$$


Note that the following terms in equation (A.15) are equal to zero:

$$
\begin{aligned}
E\left[\xi_{j, t+1}^{x}\left(\xi_{k, t+1}^{x}\right)^{2}\right] & =E\left[E\left[\xi_{j, t+1}^{x}\left(\xi_{k, t+1}^{x}\right)^{2} \mid \xi_{k, t+1}^{x}\right]\right]=E\left[0 \cdot\left(\xi_{k, t+1}^{x}\right)^{2}\right]=0, \quad \forall j, k \\
E\left[\xi_{j, t+1}^{x} \cdot \xi_{k, t+1}^{x} \cdot \xi_{l, t+1}^{x}\right] & =0, \quad \forall j, k, l .
\end{aligned}
$$

Using this, the third central moment can be rewritten as

$$
E_{t}\left[\left(J_{t+1}^{x}\right)^{3}\right]=E_{t}\left[N_{t+1}^{x} \cdot E\left[\left(\xi_{j, t+1}^{x}\right)^{3}\right]\right]=E_{t}\left[N_{t+1}^{x} \cdot\left(-2 \mu_{x}^{3}\right)\right]=-2 \mu_{x}^{3}\left(l_{0}^{x}+l_{1}^{x} \sigma_{t}\right)
$$

Thus, the conditional moments of $x_{t+1}$ are:

$$
\begin{aligned}
E_{t}\left[x_{t+1}\right] & =\rho_{x} x_{t}+E_{t}\left[\varphi_{e} \sqrt{\sigma_{t}} \varepsilon_{t+1}^{x}\right]+E_{t}\left[J_{t+1}^{x}\right]=0 \\
V_{t}\left[x_{t+1}\right] & =E_{t}\left[\left(\varphi_{e} \sqrt{\sigma_{t}} \varepsilon_{t+1}^{x}+J_{t+1}^{x}\right)^{2}\right]=\varphi_{e}^{2} \sigma_{t} E_{t}\left[\left(\varepsilon_{t+1}^{x}\right)^{2}\right]+E_{t}\left[\left(J_{t+1}^{x}\right)^{2}\right] \\
& =\varphi_{e}^{2} \sigma_{t}+\left(l_{0}^{x}+l_{1}^{x} \sigma_{t}\right) \mu_{x}^{2} \\
E_{t}\left[\left(x_{t+1}-E_{t}\left[x_{t+1}\right]\right)^{3}\right] & =E_{t}\left[\left(\varphi_{e} \sqrt{\sigma_{t}} \varepsilon_{t+1}^{x}+J_{t+1}^{x}\right)^{3}\right]=\varphi_{e}^{3} \sigma_{t}^{3 / 2} E_{t}\left[\left(\varepsilon_{t+1}^{x}\right)^{3}\right]+E_{t}\left[\left(J_{t+1}^{x}\right)^{3}\right] \\
& =-2 \mu_{x}^{3}\left(l_{0}^{x}+l_{1}^{x} \sigma_{t}\right) .
\end{aligned}
$$

This implies that the conditional skewness of $x_{t+1}$ is:

$$
\operatorname{Skew}_{t}\left[x_{t+1}\right]=-\frac{2 \mu_{x}^{3} \cdot l_{1}^{x} \cdot \sigma_{t}}{\left[\varphi_{e}^{2} \sigma_{t}+\left(l_{0}^{x}+l_{1}^{x} \sigma_{t}\right) \mu_{x}^{2}\right]^{3 / 2}}
$$

Setting $l_{0}^{x}=0$ delivers the expression for skewness reported in main text.

\section{A.7 Time series properties for alternative data configurations}

In Table A.1, we conduct our analysis of six alternative datasets for the construction of the crosssectional moments of expected GDP growth rates. Specifically, the first three panels of Table A.1 report the results for: 1) the Livingston Survey combined with the Blue Chips Survey; 2) the Livingston Survey alone; and 3) the Blue Chips Survey alone. A further break down the dataset by excluding from the most comprehensive dataset: 4) the Livingston Survey data after 1998 (i.e. the 
years for which the cross-sectional size drops by $30 \%$ or more); 5) the Livingston Survey semesters during which the cross-sectional size was smaller that 20; and 6) the Livingston Survey semesters during which the cross-sectional size was smaller that 30 . In this way, we aim at providing the broadest outlook of our dataset.

\section{A.8 Robustness of predictive regressions}

In this section, we report the robustness of our findings concerning the predictive regressions featured in section 2. Tables A.2- A.4 contain our results for alternative configurations of the analysts' forecasts. Tables A.5-A.8 report our results with bootstrapped standard errors. Tables A.9-A.12 report the results including the cross-sectional kurtosis. Table A.13 reports the results obtained by removing the forecasts of analysts whose affiliation overlaps between the Livingston and the Blue Chips surveys. We refer the reader to the tables' notes for additional details.

\section{A.9 Proofs of lemmas}

Proof of Lemma A.1.1. By definition:

$E \exp \left\{\kappa_{1} z+\kappa_{2} z^{2}\right\}=\int_{-\infty}^{+\infty} \exp \left\{\kappa_{1} z+\kappa_{2} z^{2}\right\}\left[\frac{1}{\sigma \pi} \exp \left\{-\frac{(z-\mu)^{2}}{2 \sigma^{2}}\right\} \cdot\left(\int_{-\infty}^{\nu \frac{(z-\mu)}{\sigma}} \exp \left\{-\frac{t^{2}}{2}\right\} d t\right)\right] d z$

where the term in the square brackets corresponds to the probability distribution function of a skewnormal. It follows that:

$$
\begin{aligned}
E \exp \left\{\kappa_{1} z+\kappa_{2} z^{2}\right\}= & \int_{-\infty}^{+\infty} \frac{1}{\sigma \pi} \exp \left\{-\frac{z^{2}-2\left(\frac{\kappa_{1} \sigma^{2}+\mu}{1-2 \kappa_{2} \sigma^{2}}\right) z+\frac{\mu^{2}}{1-2 \kappa_{2} \sigma^{2}}}{2\left(\frac{\sigma^{2}}{1-2 \kappa_{2} \sigma^{2}}\right)}\right\} \cdot\left(\int_{-\infty}^{\nu \frac{(z-\mu)}{\sigma}} \exp \left\{-\frac{t^{2}}{2}\right\} d t\right) d z \\
= & \exp \left\{\frac{\frac{\left(\kappa_{1} \sigma^{2}+\mu\right)^{2}}{1-2 \kappa_{2} \sigma^{2}}-\mu^{2}}{2 \sigma^{2}}\right\} \cdot \int_{-\infty}^{+\infty} \frac{1}{\sigma \pi} \exp \left\{-\frac{\left(z-\left(\frac{\kappa_{1} \sigma^{2}+\mu}{1-2 \kappa_{2} \sigma^{2}}\right)\right)^{2}}{2\left(\frac{\sigma^{2}}{1-2 \kappa_{2} \sigma^{2}}\right)}\right\} . \\
& \left(\int_{-\infty}^{\nu \frac{(z-\mu)}{\sigma}} \exp \left\{-\frac{t^{2}}{2}\right\} d t\right) d z .
\end{aligned}
$$

Apply the following change of variable

$$
y=\frac{z-\left(\frac{\kappa_{1} \sigma^{2}+\mu}{1-2 \kappa_{2} \sigma^{2}}\right)}{\sigma} \cdot \sqrt{1-2 \kappa_{2} \sigma^{2}}
$$




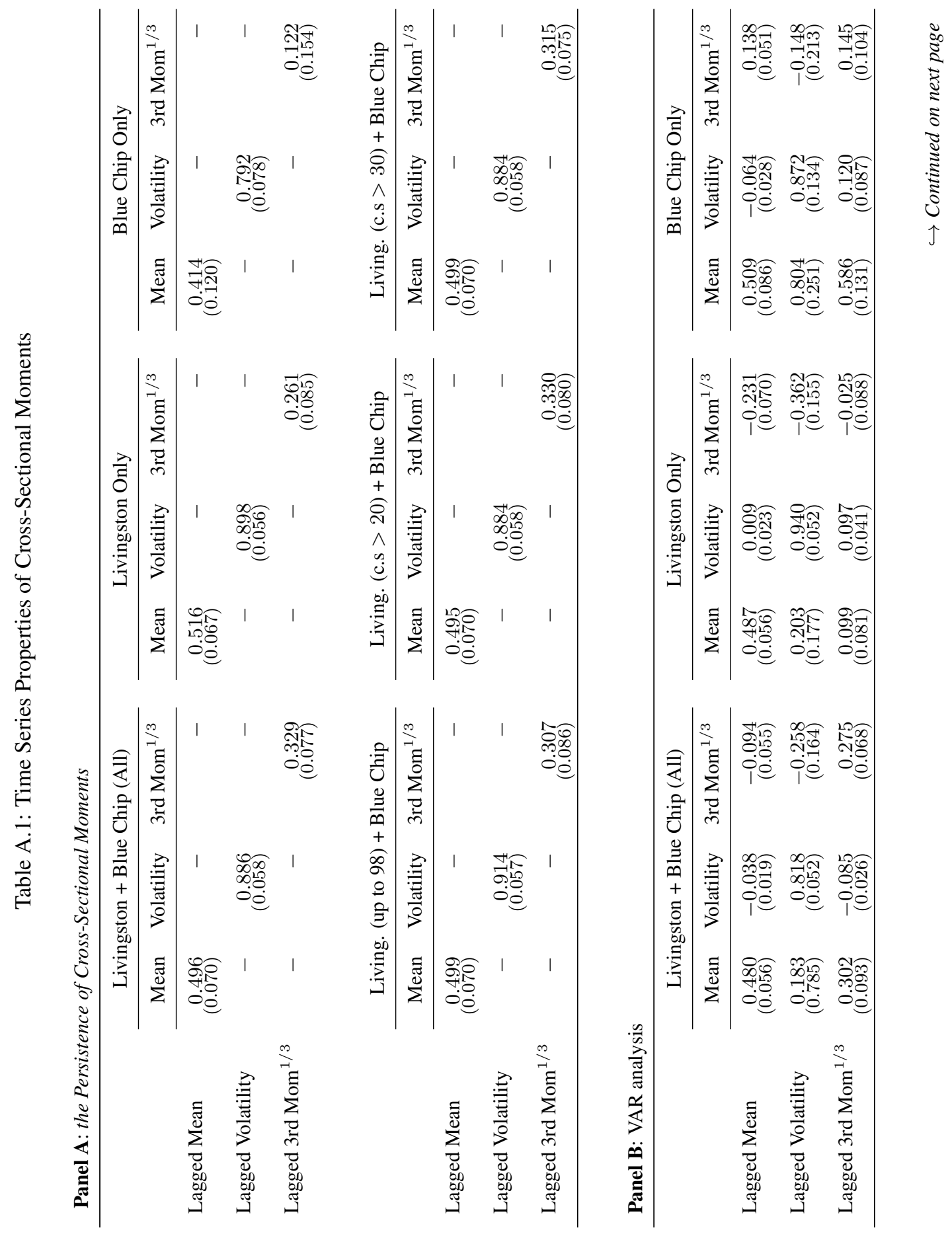




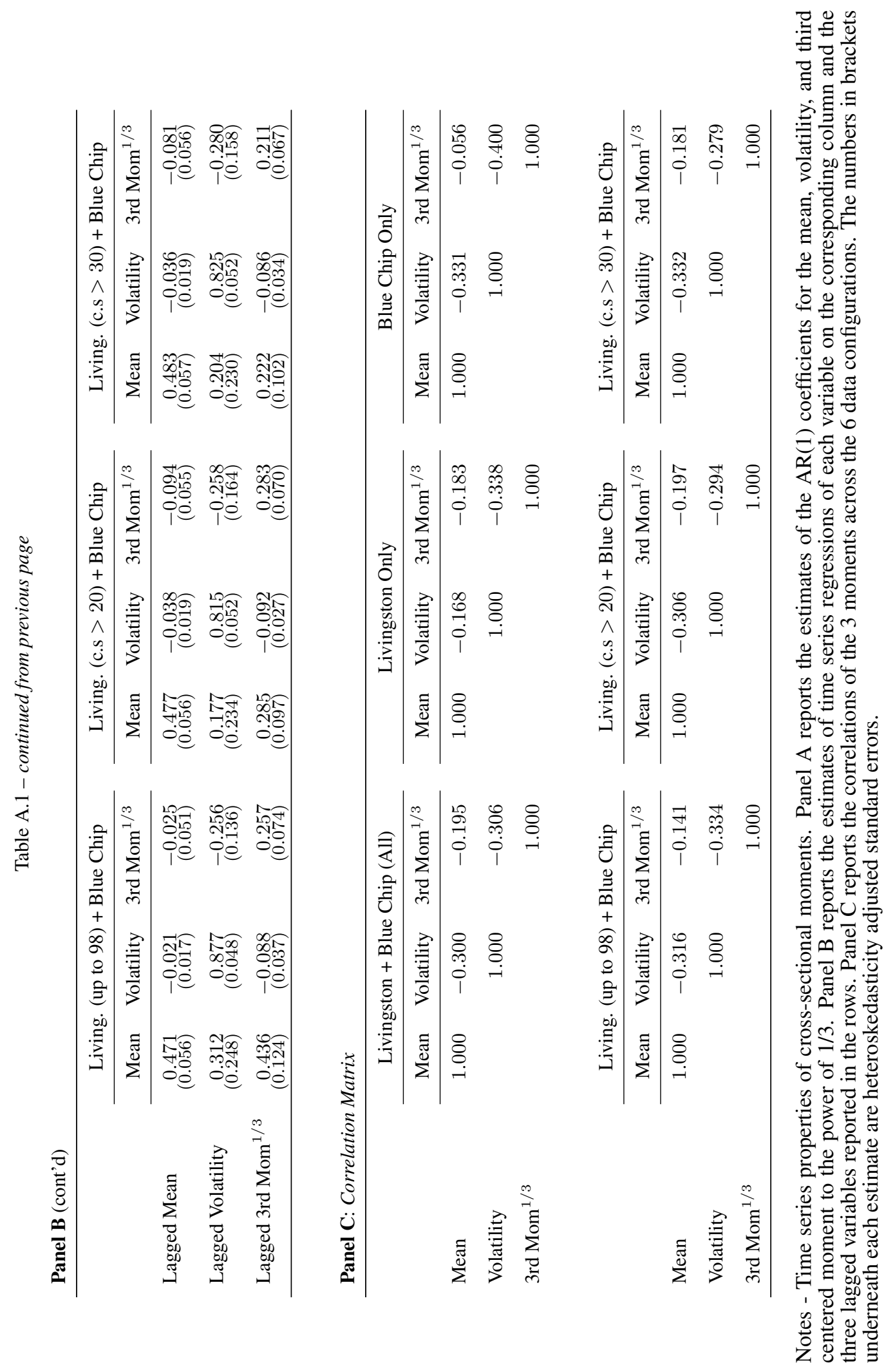




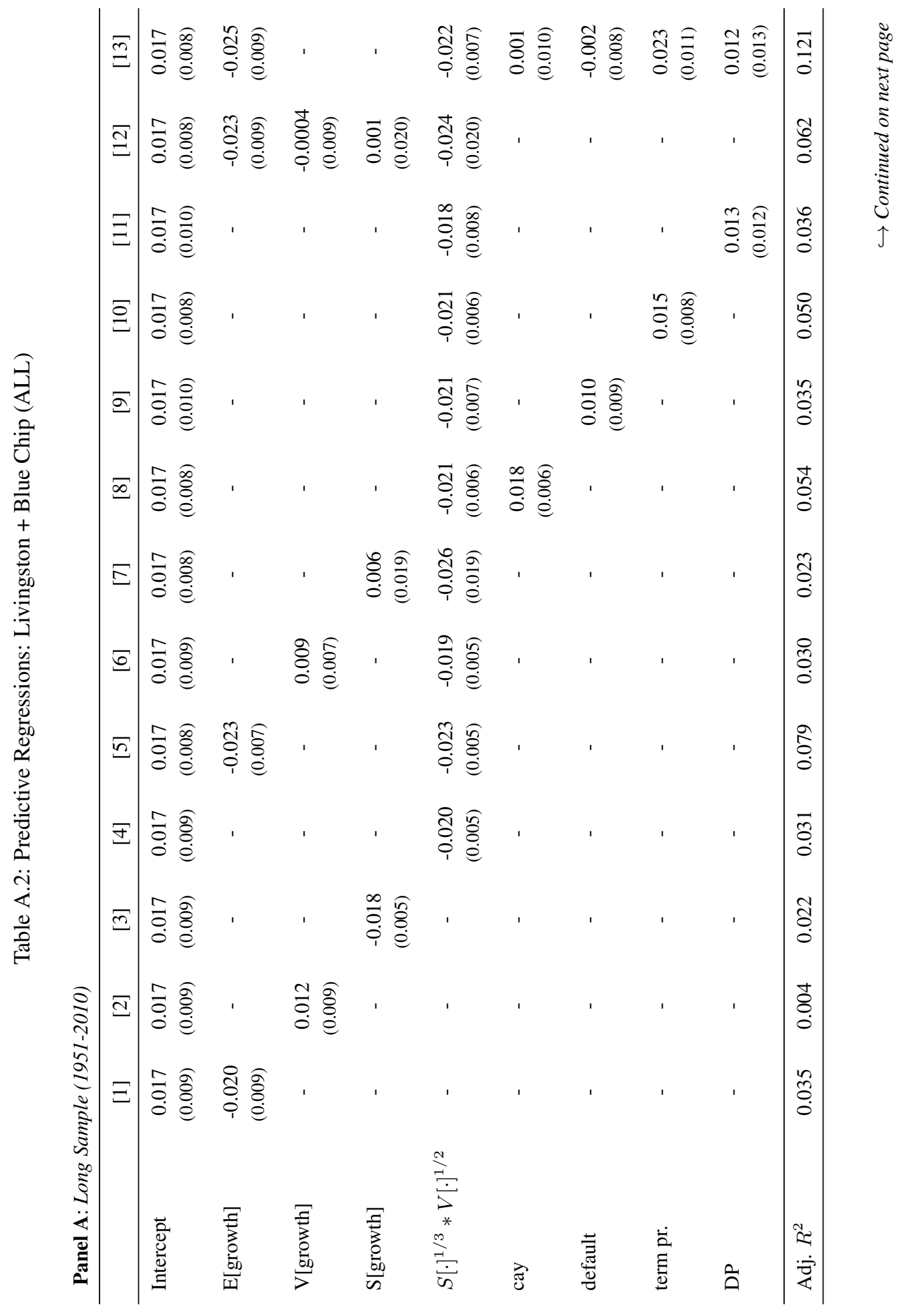




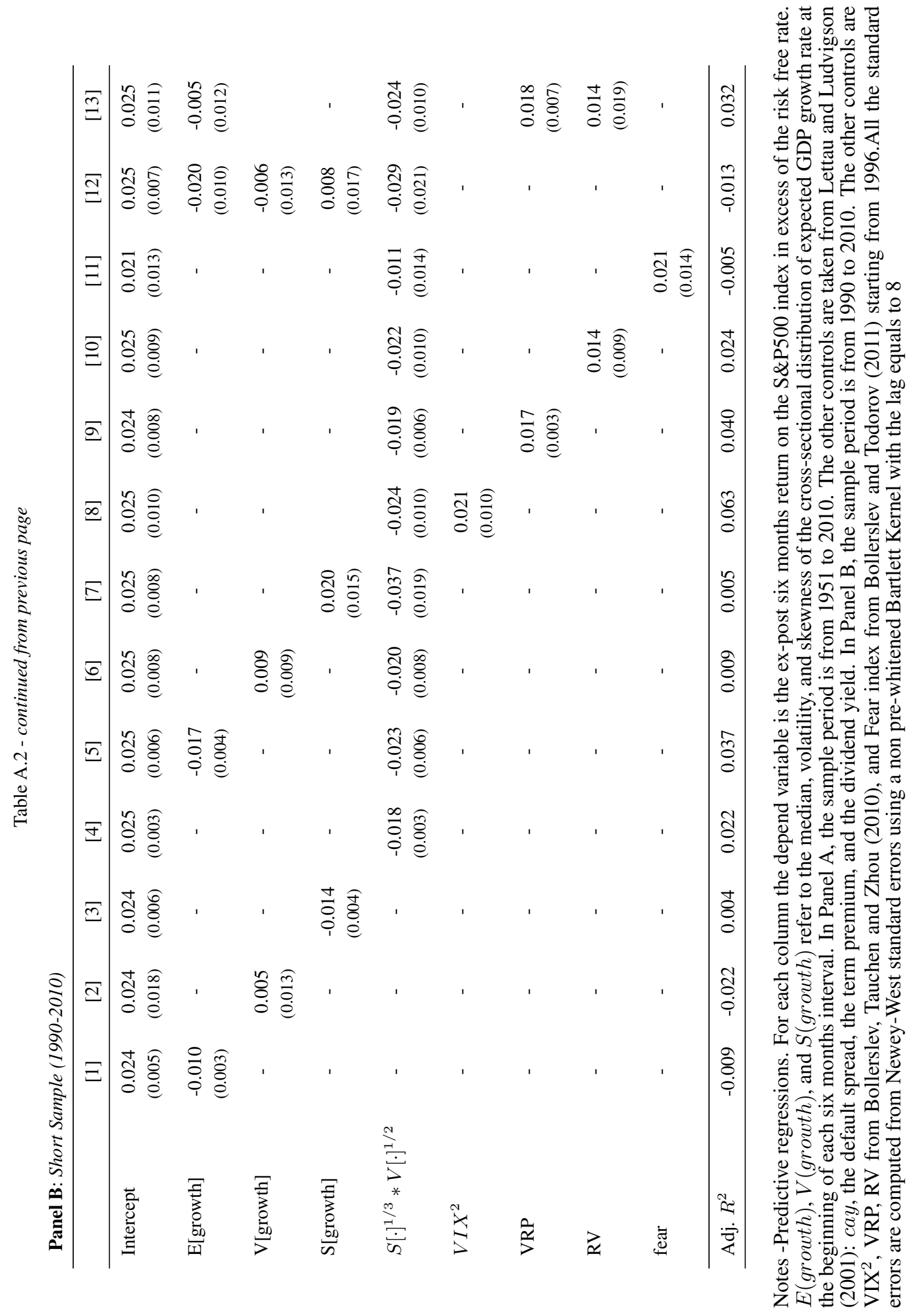




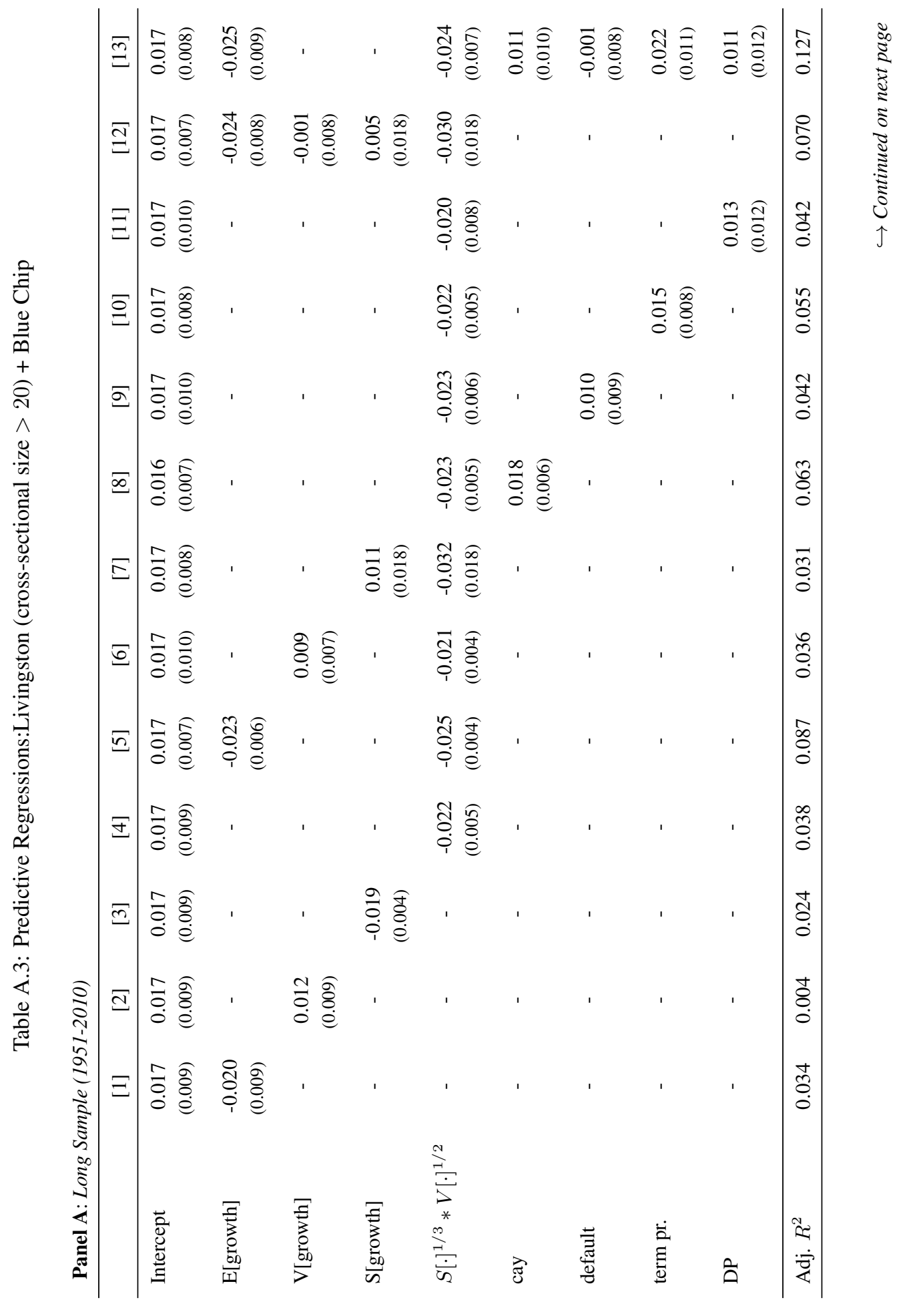




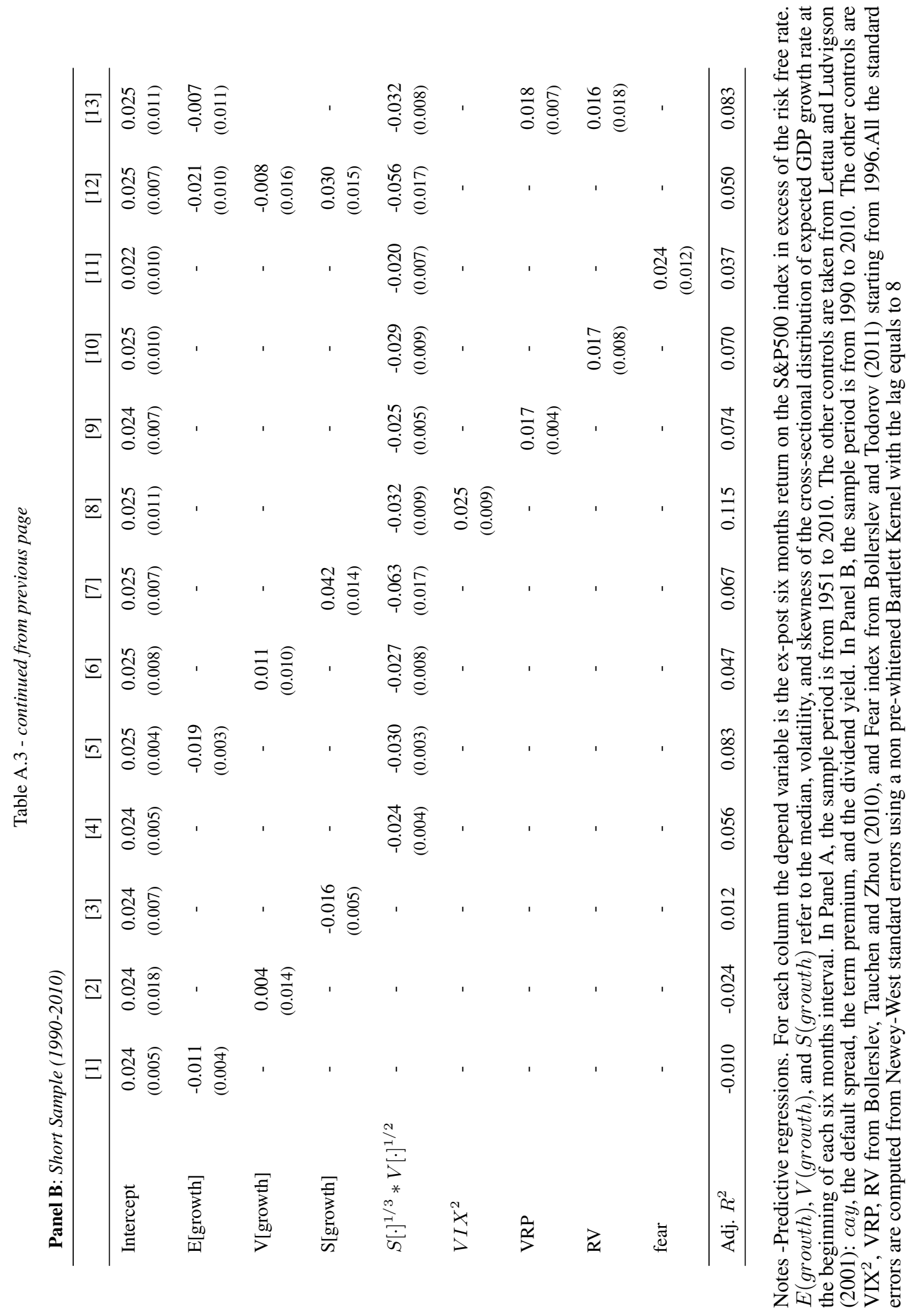




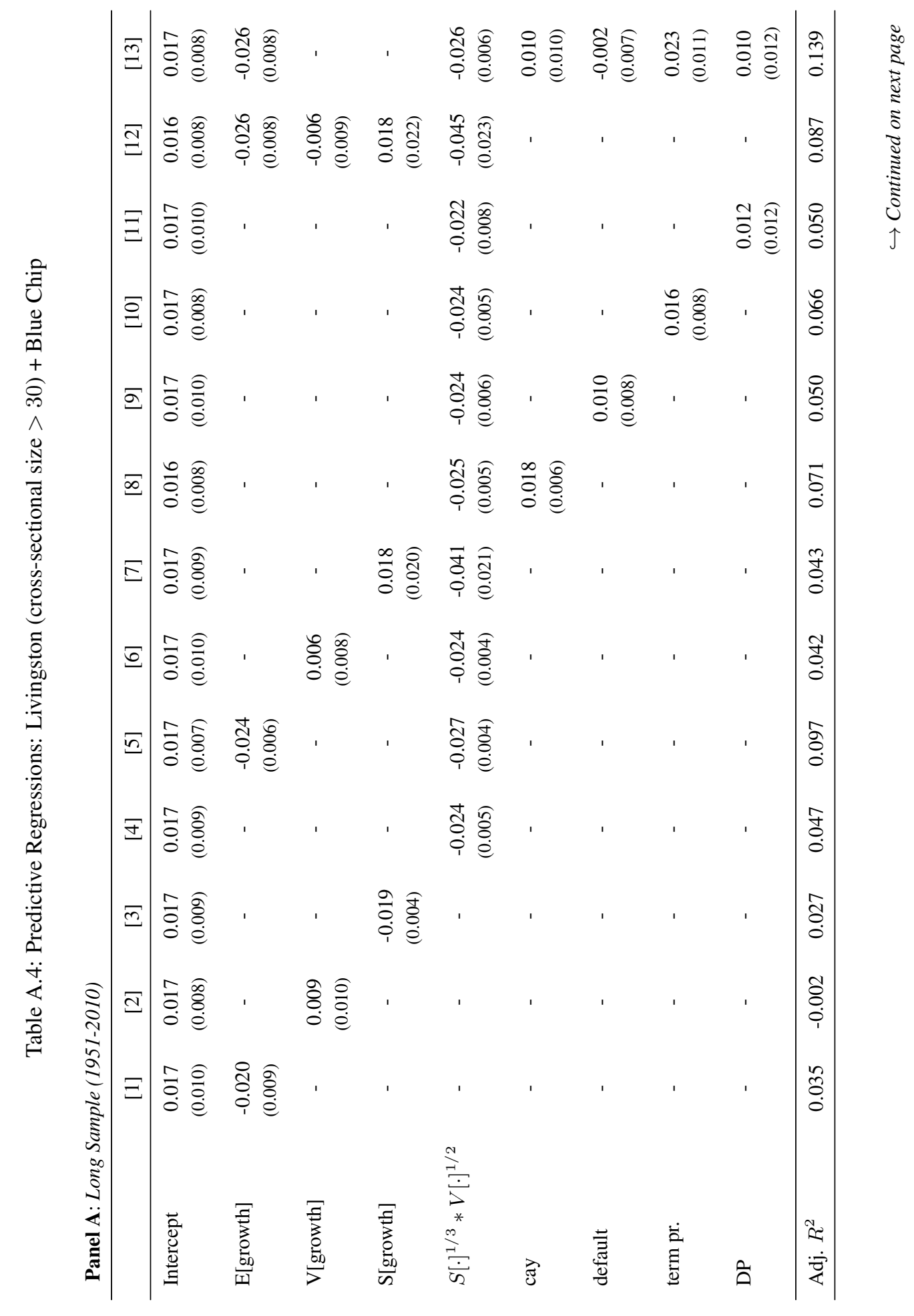




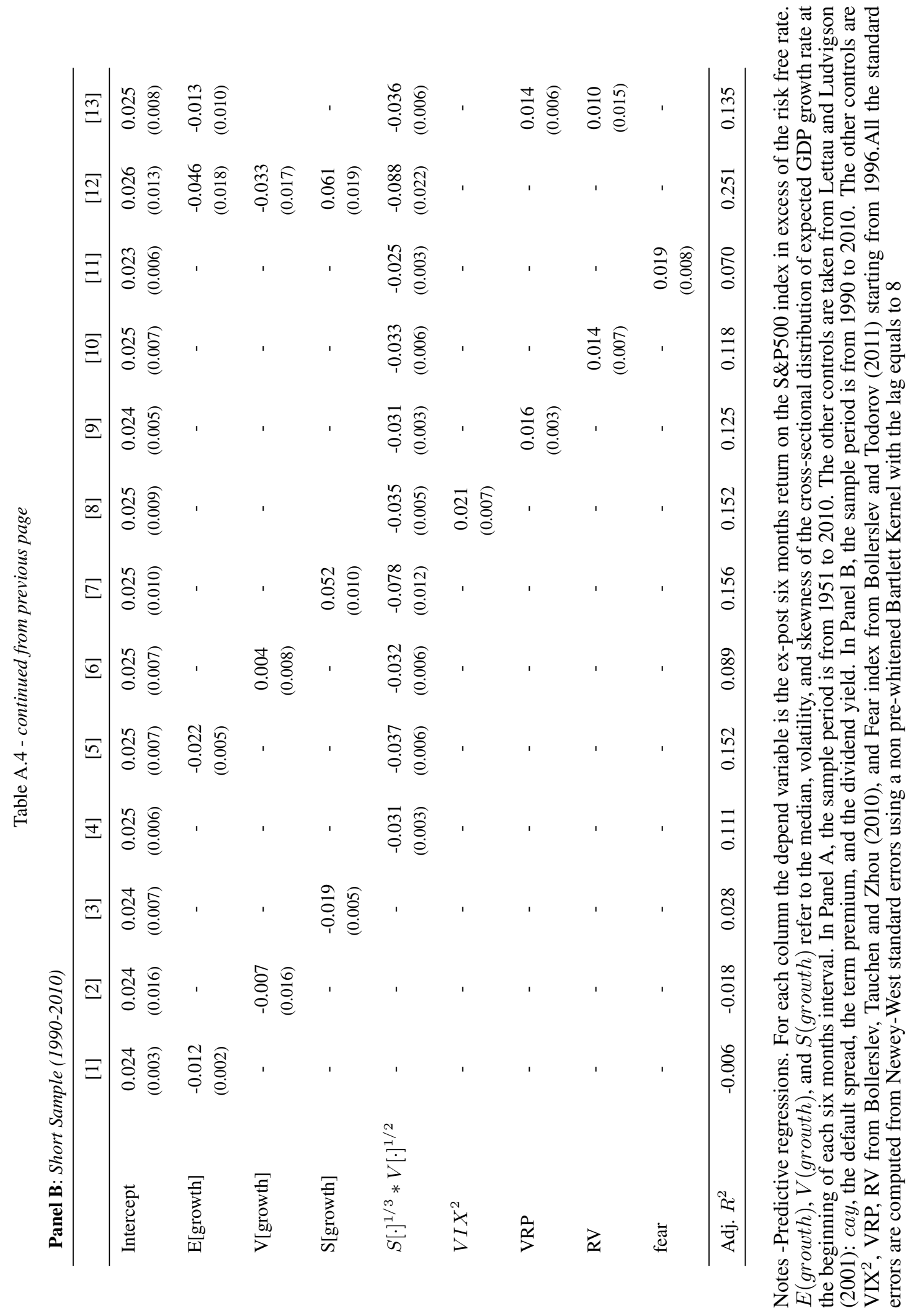




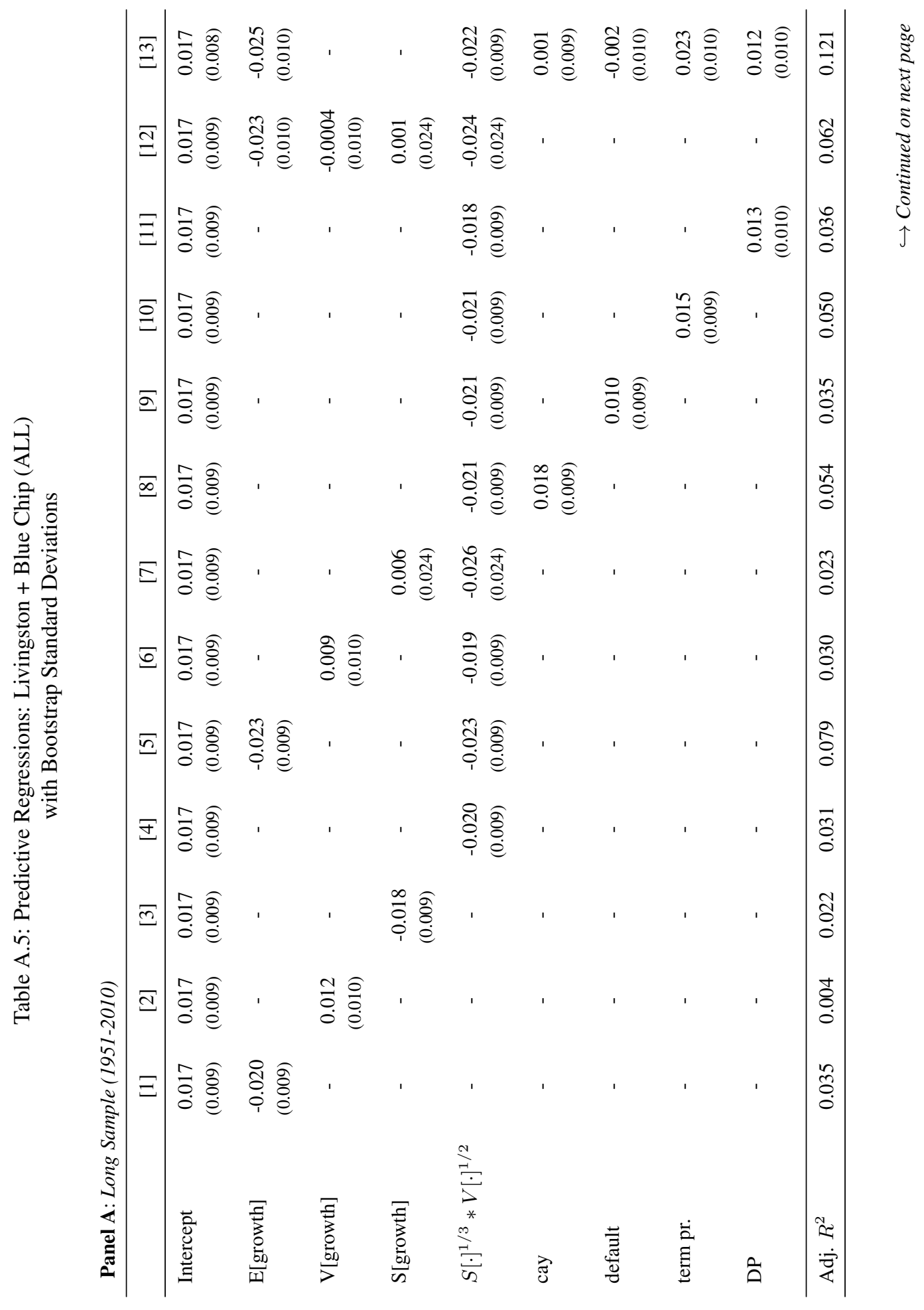




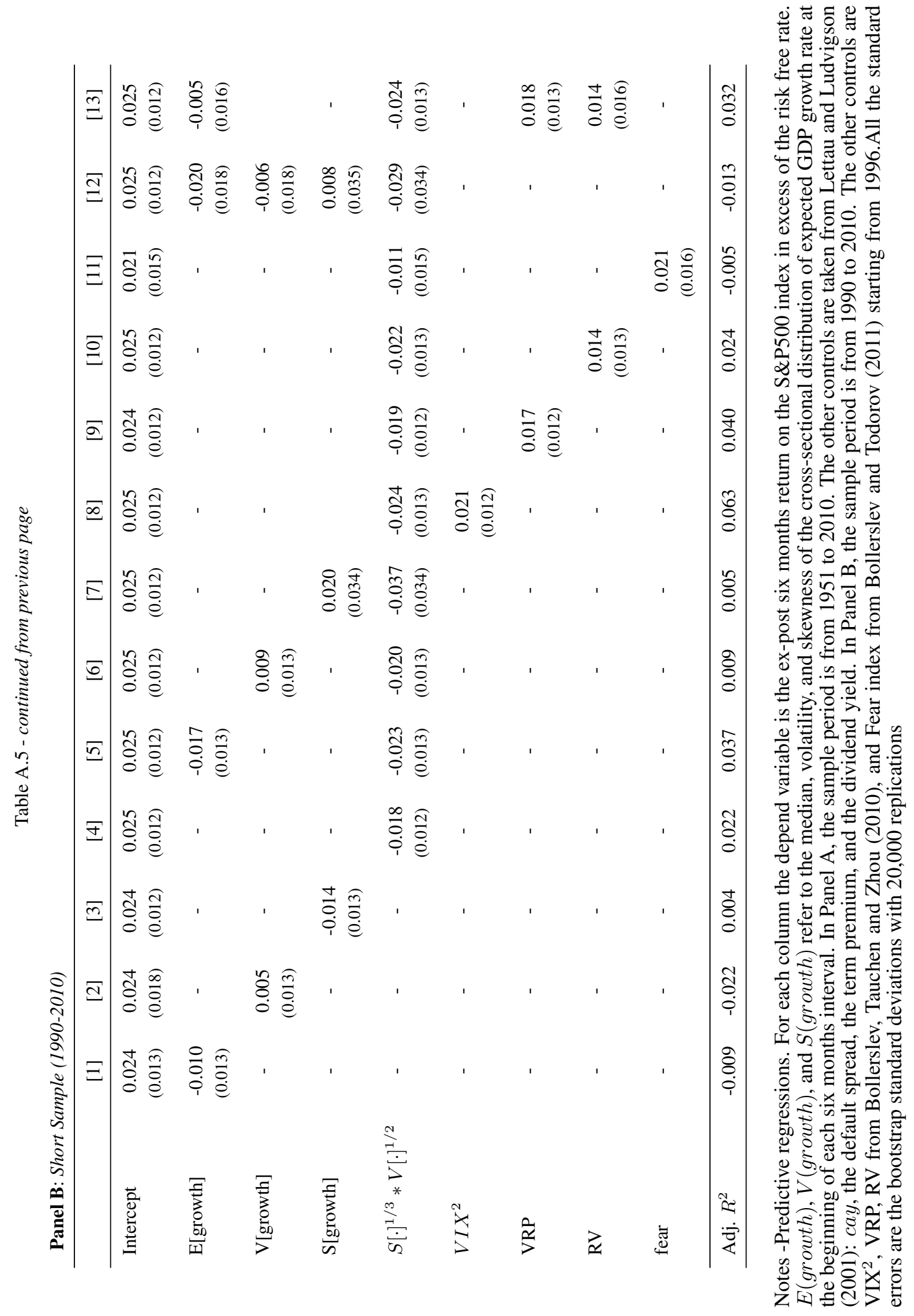




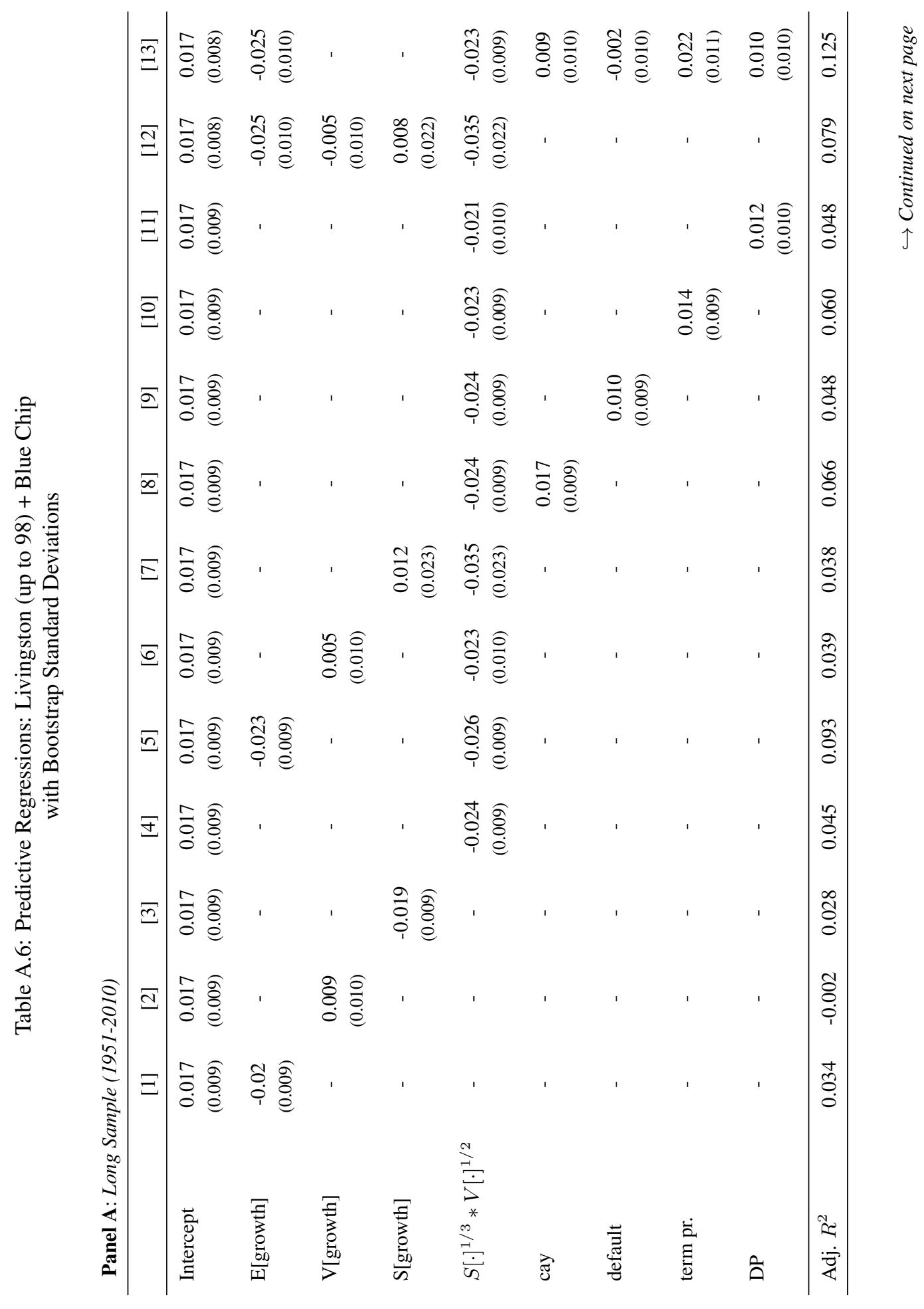




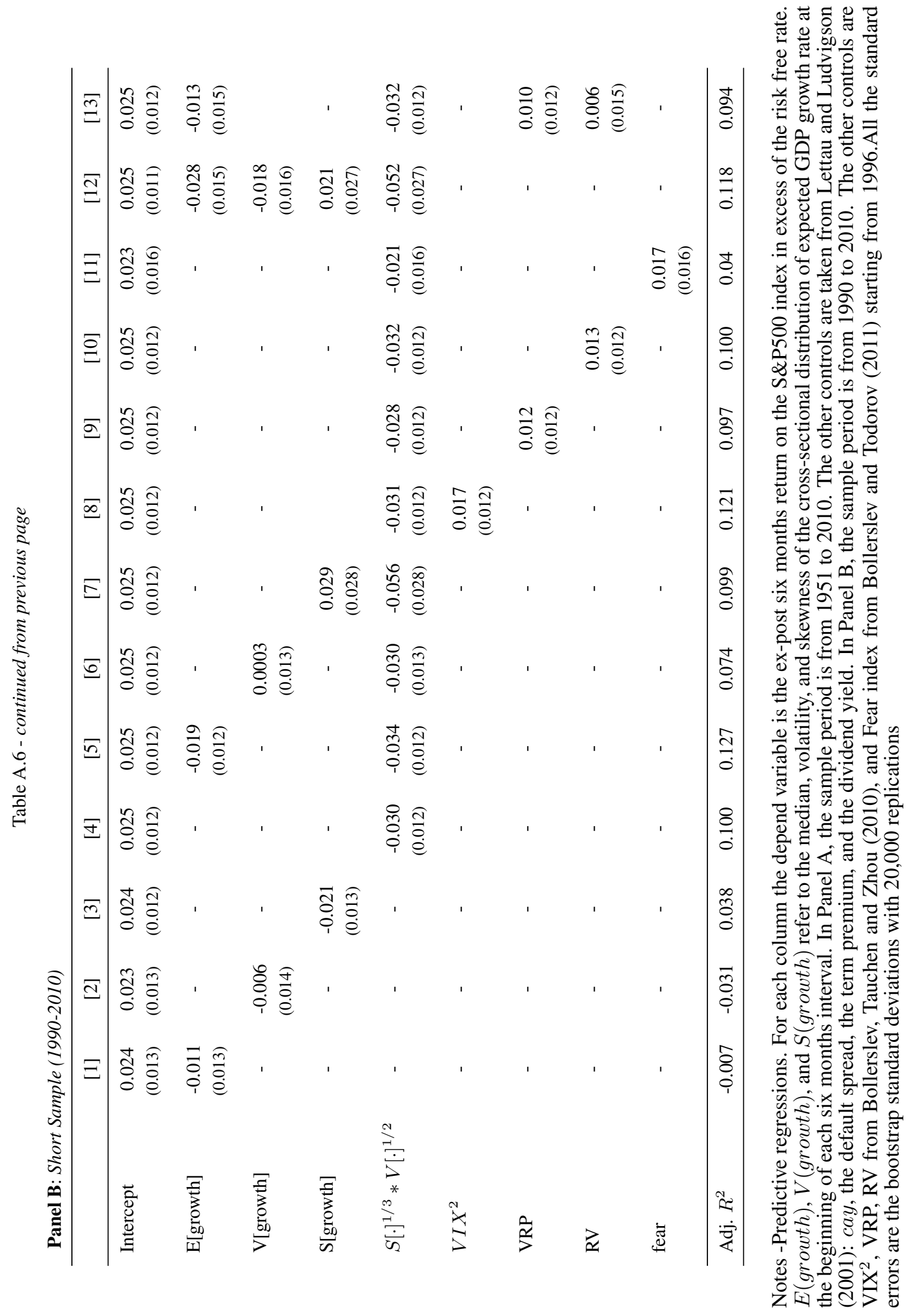




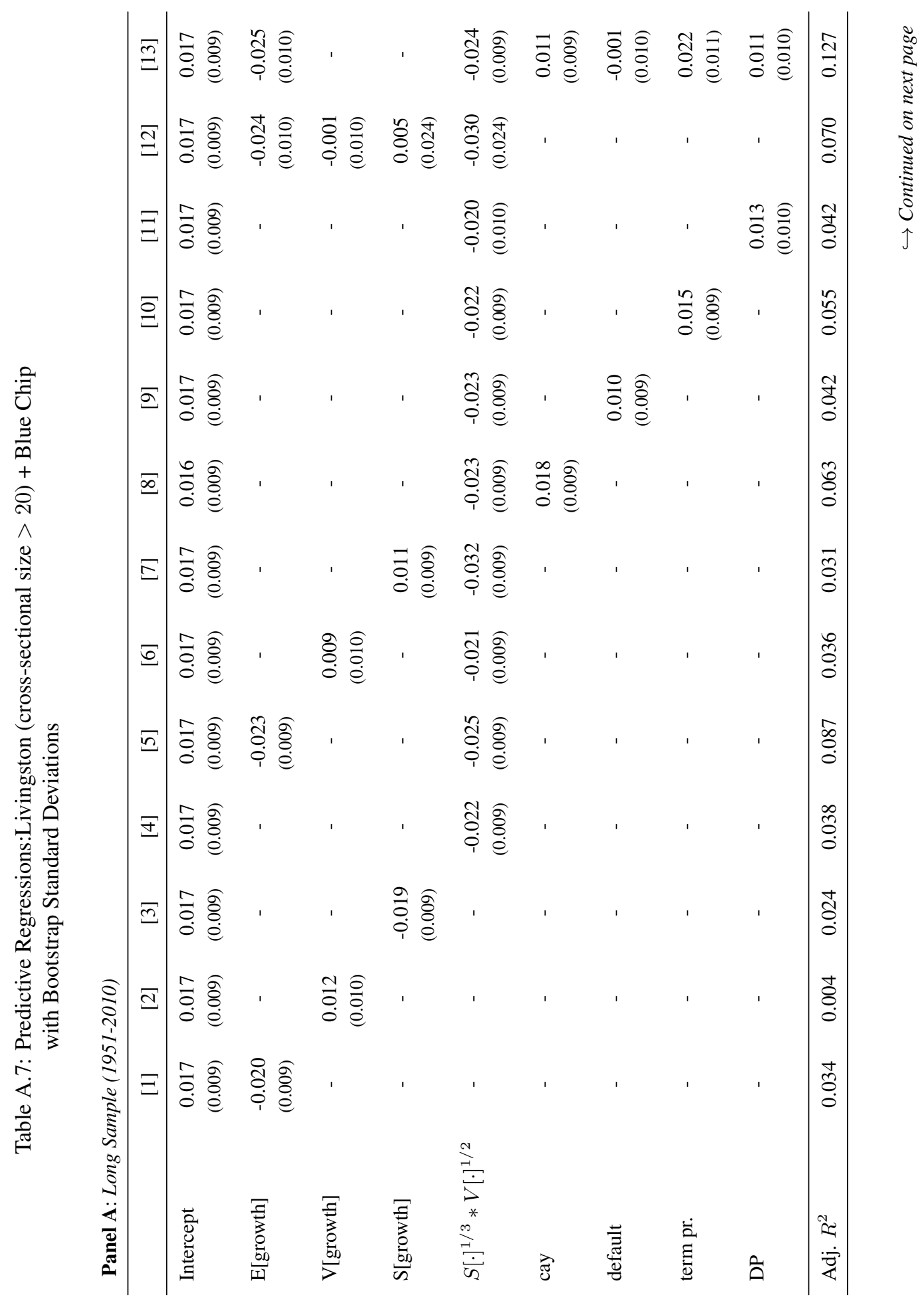




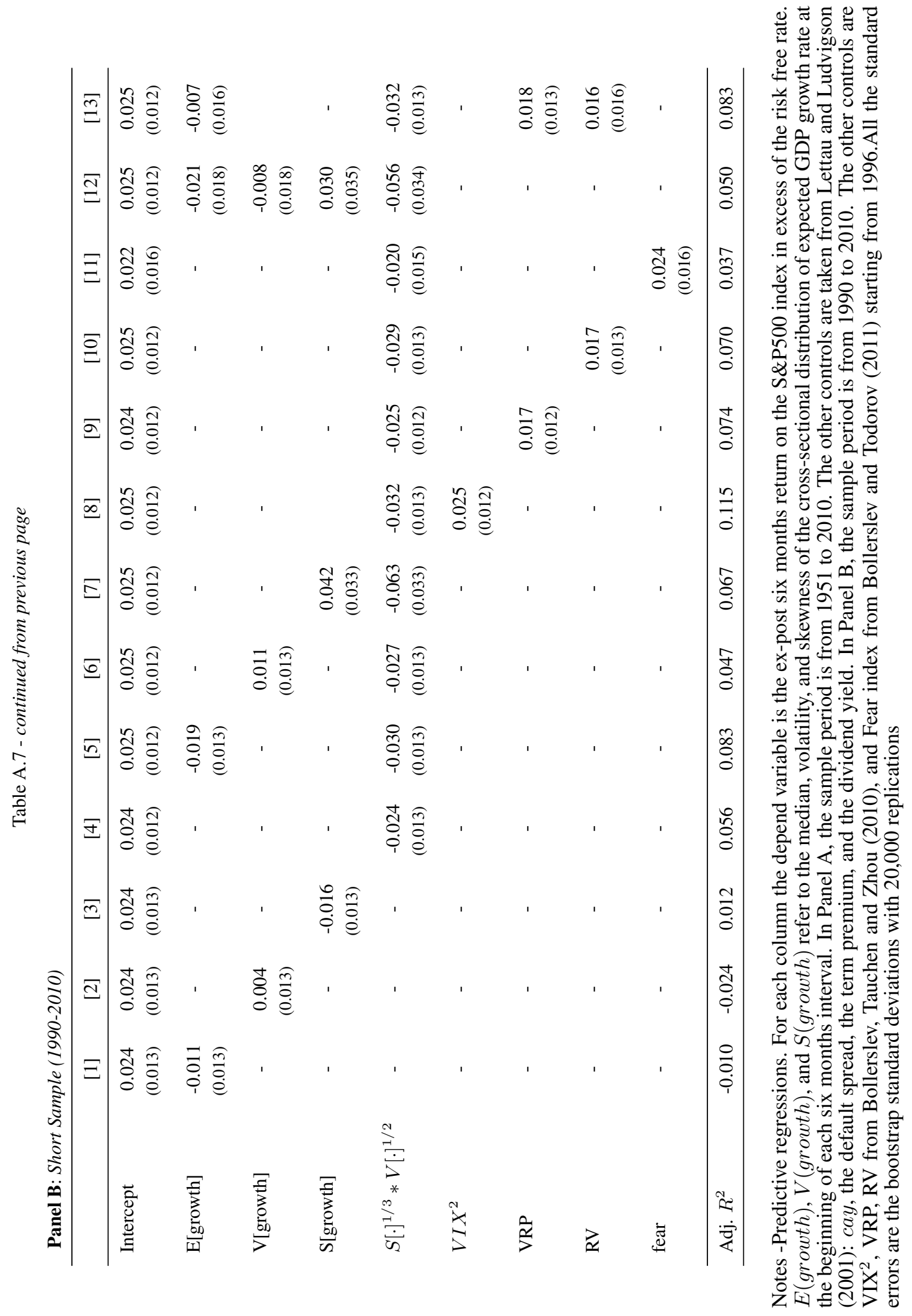




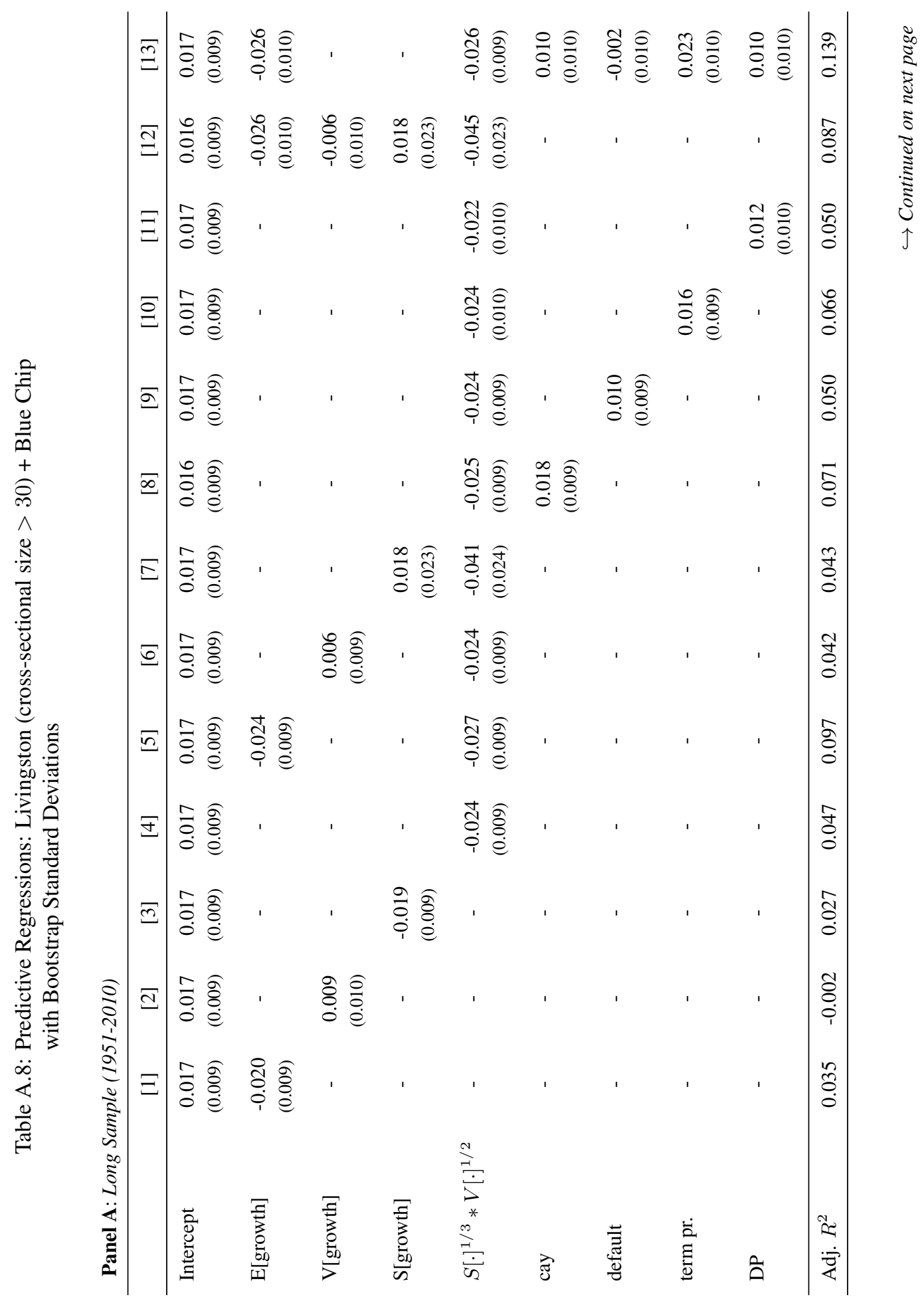




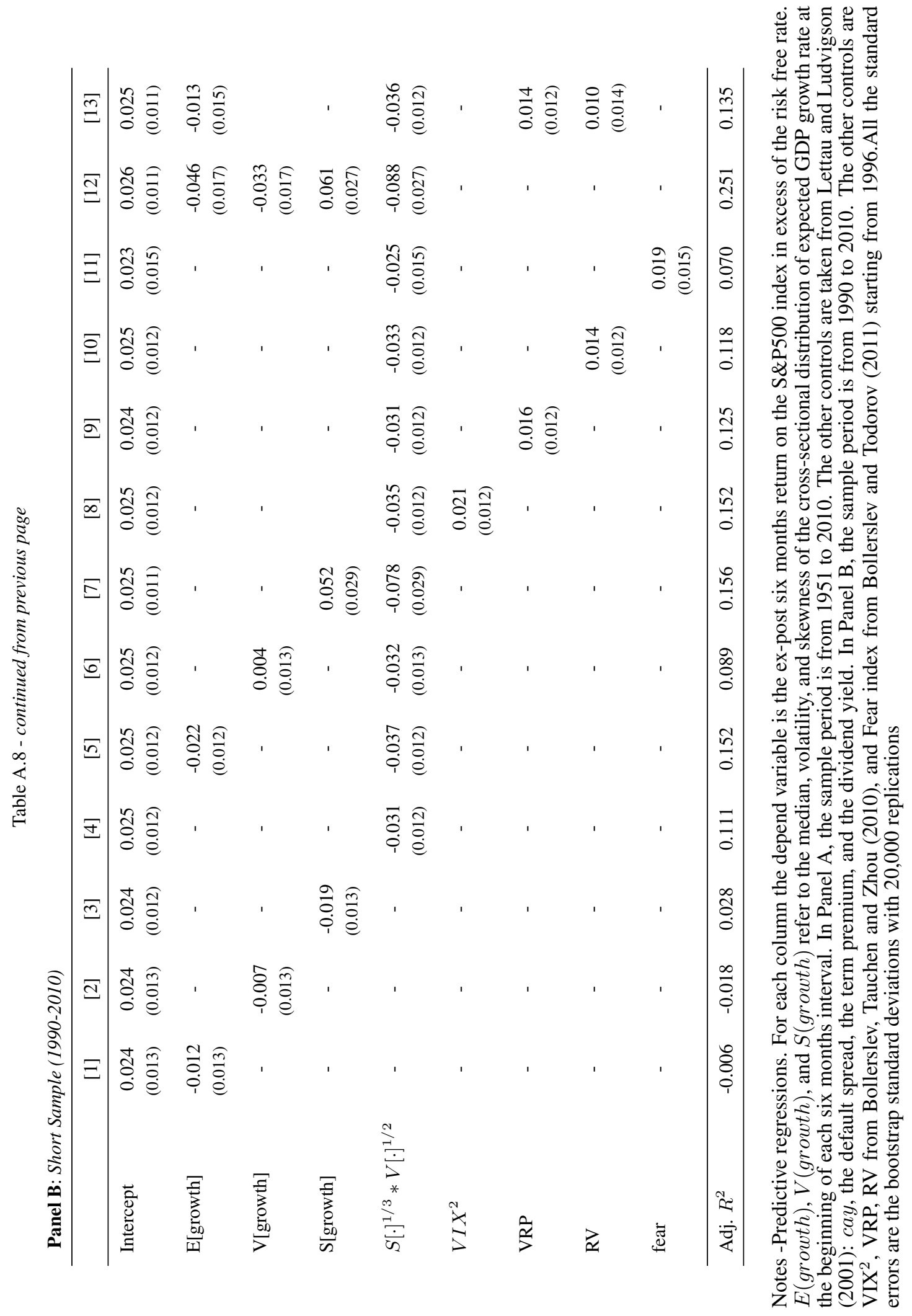




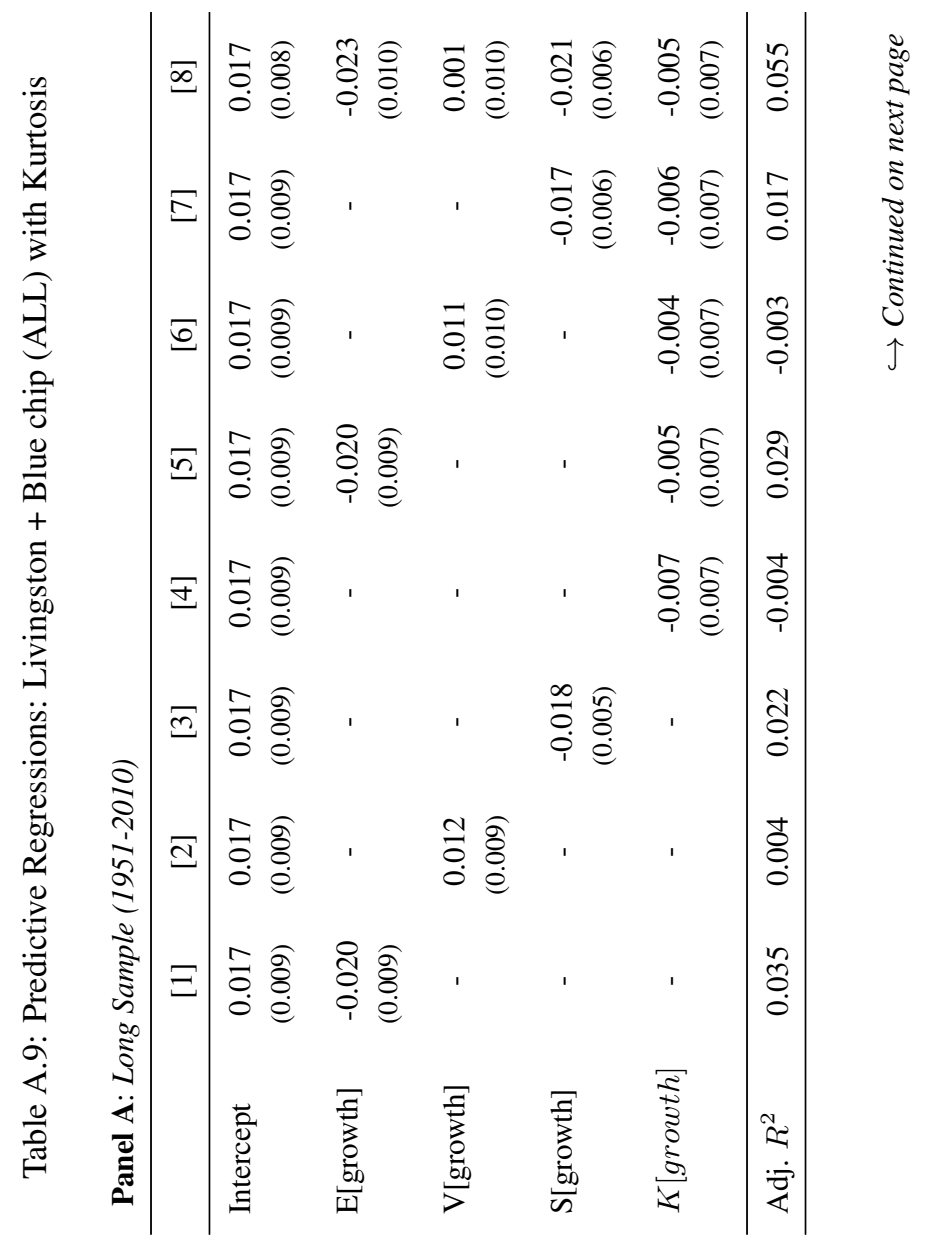




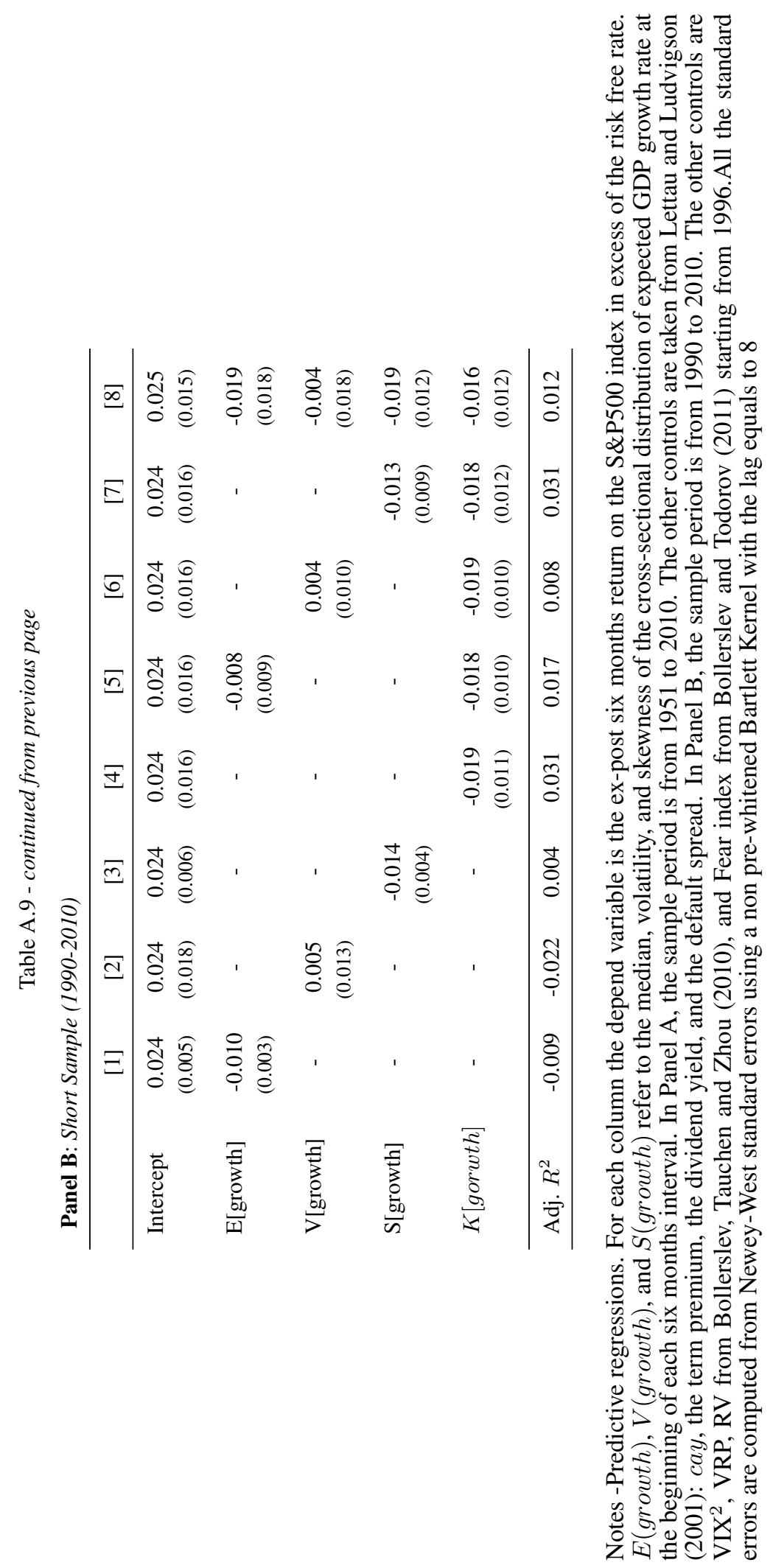




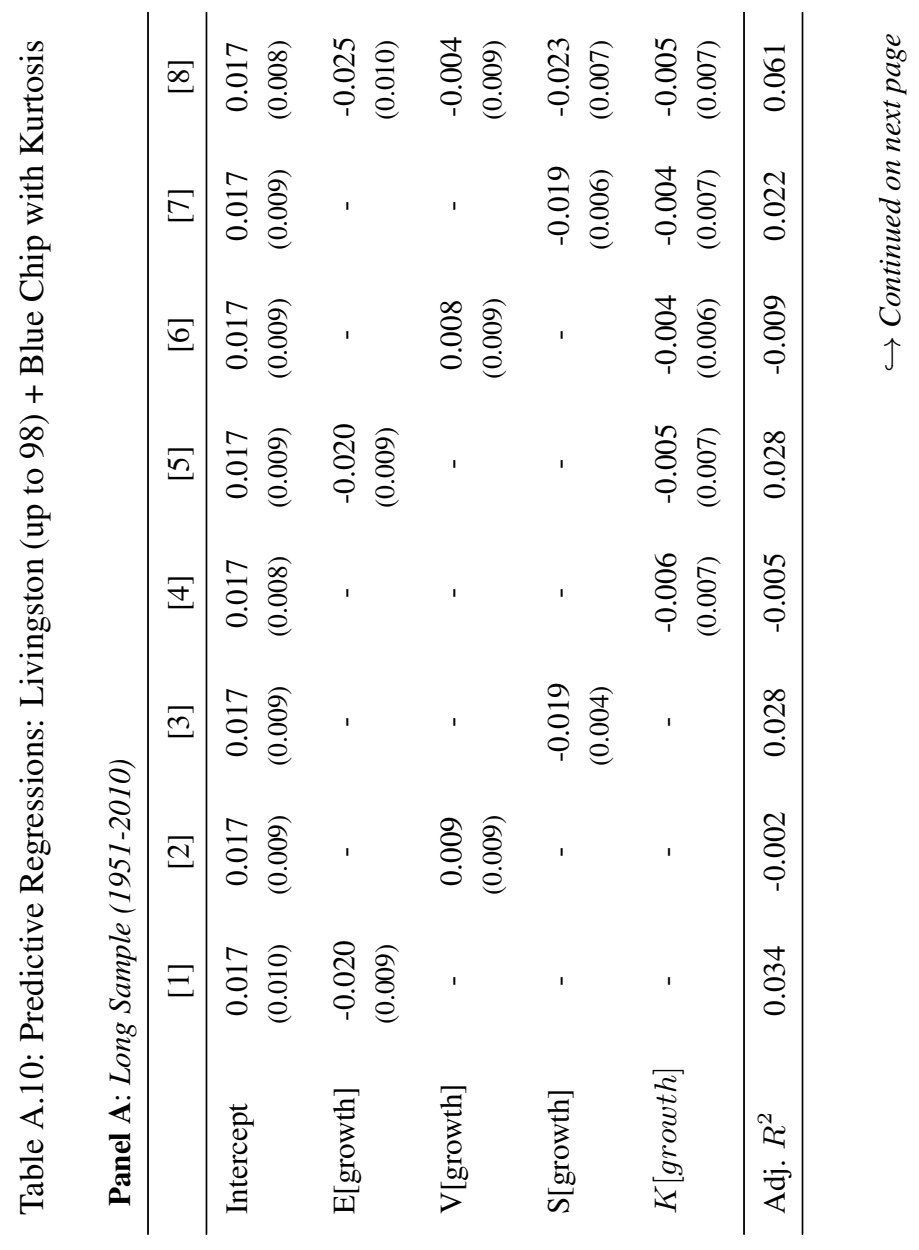




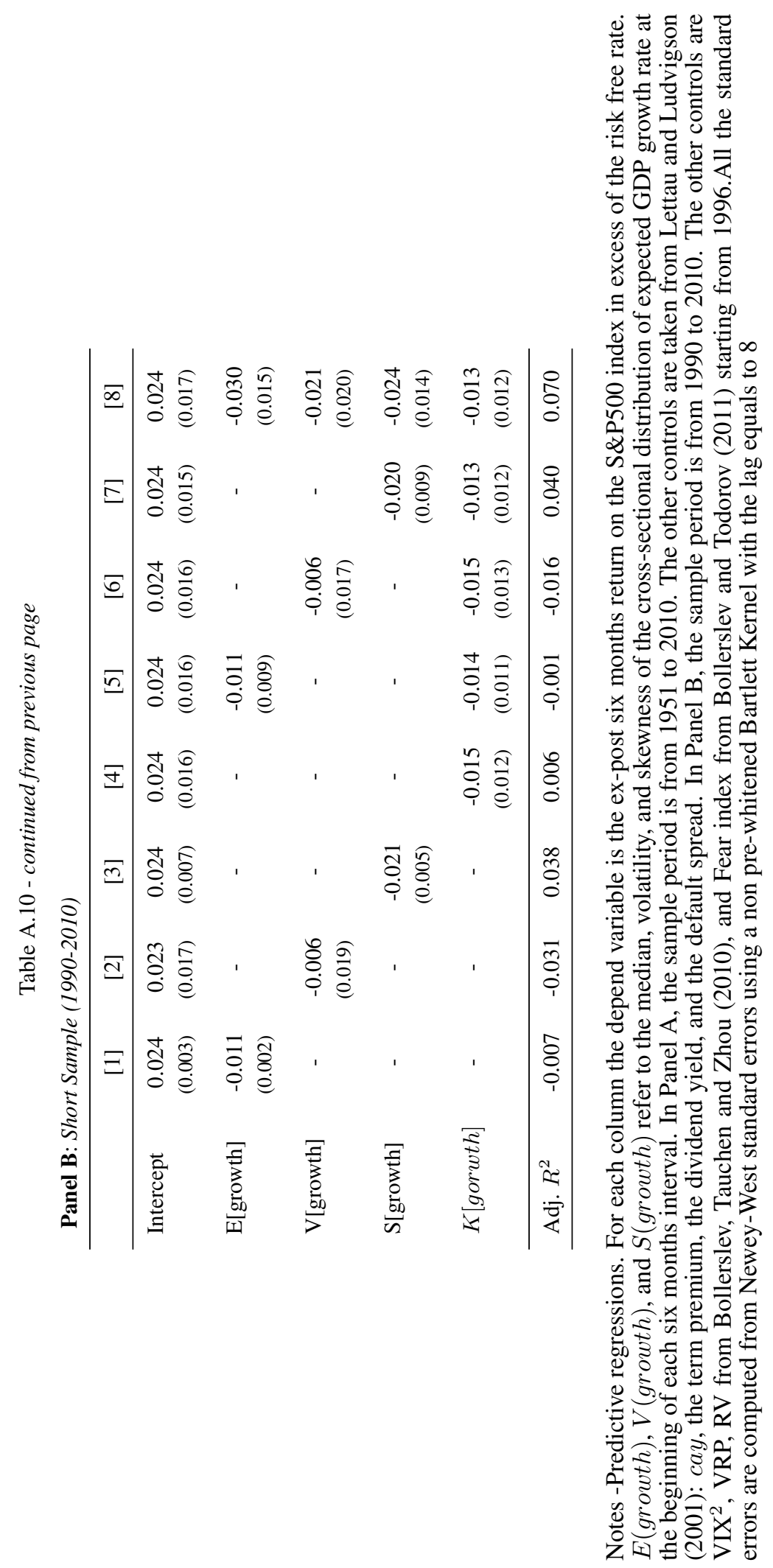




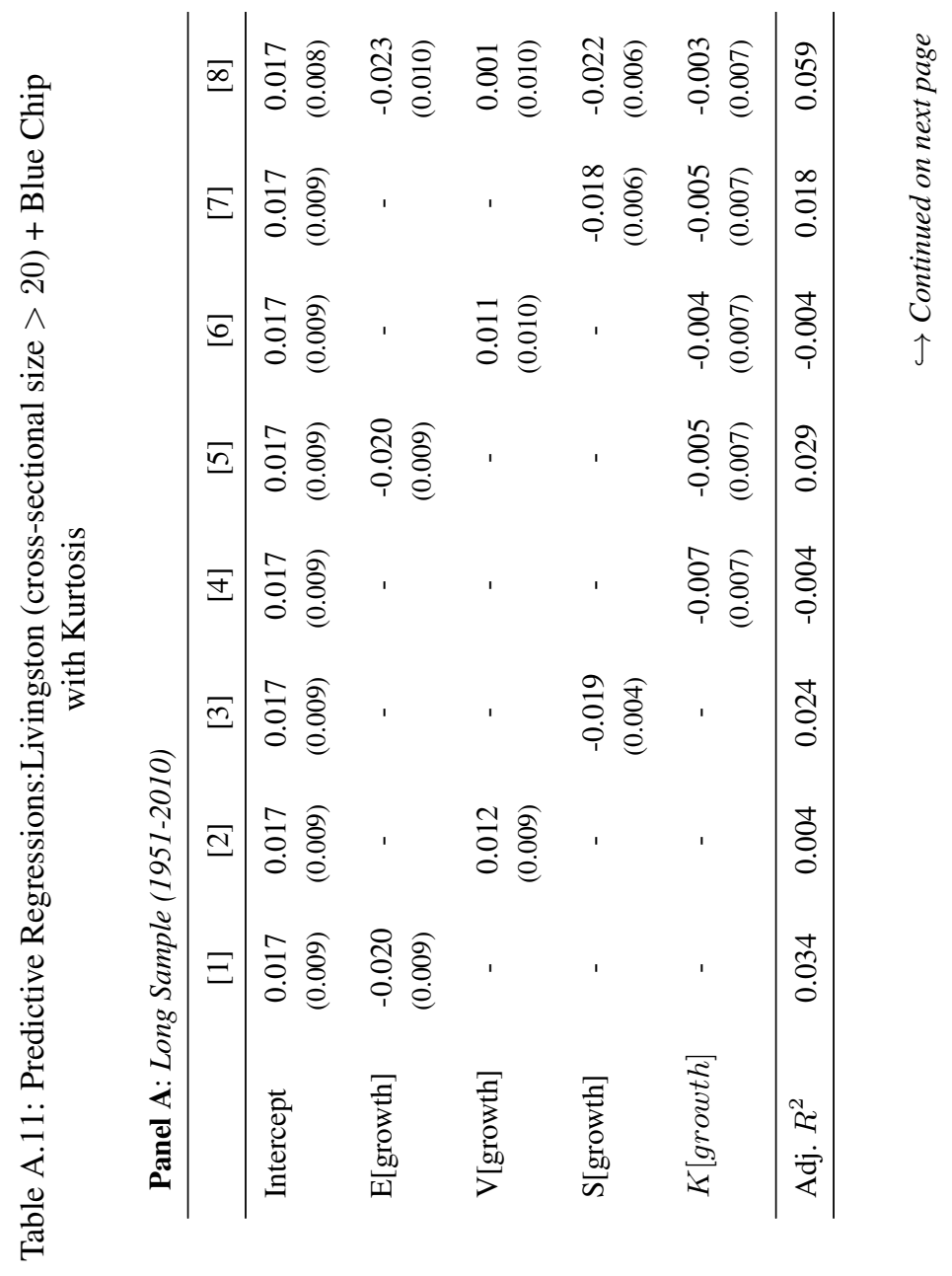




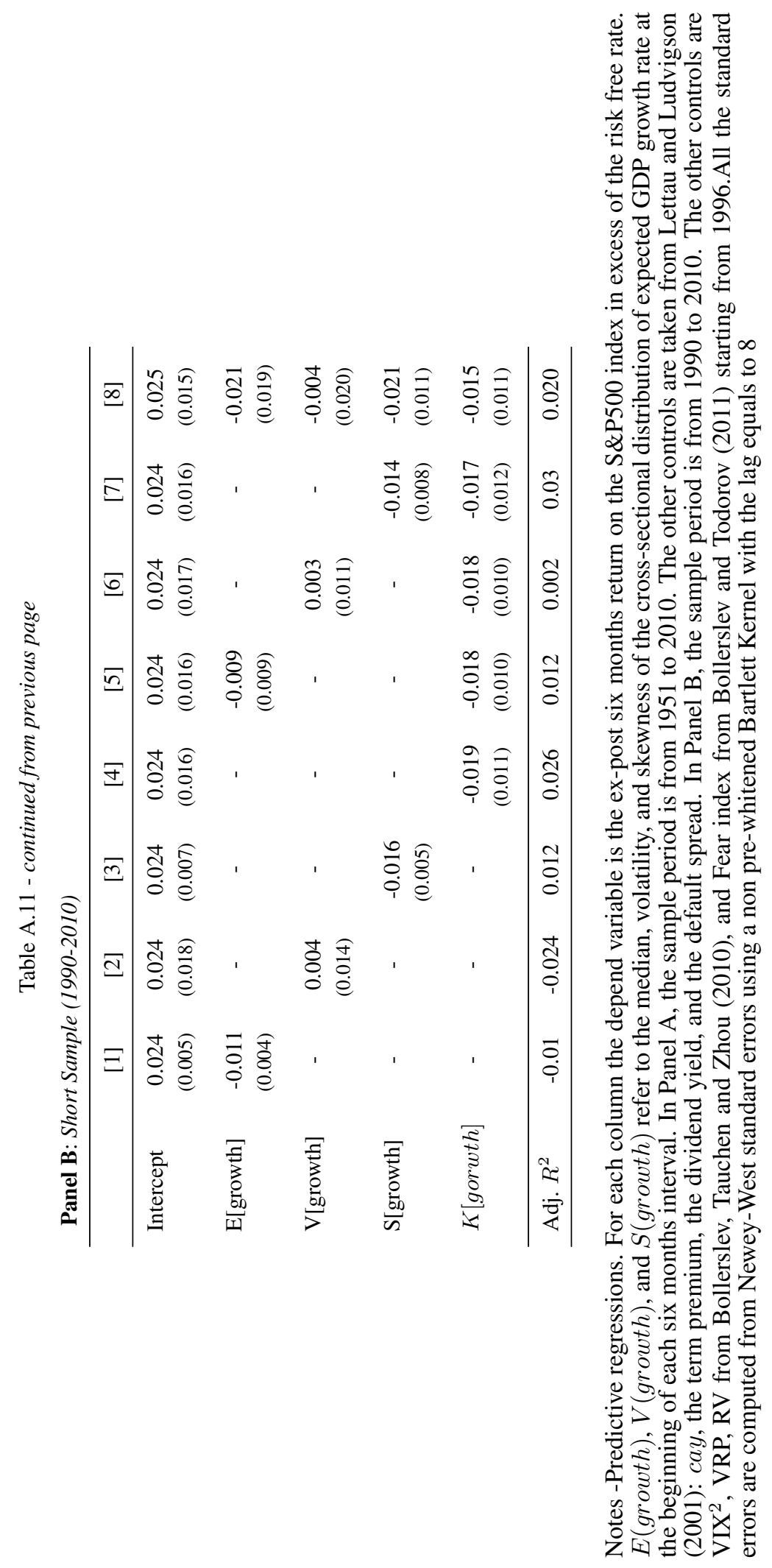




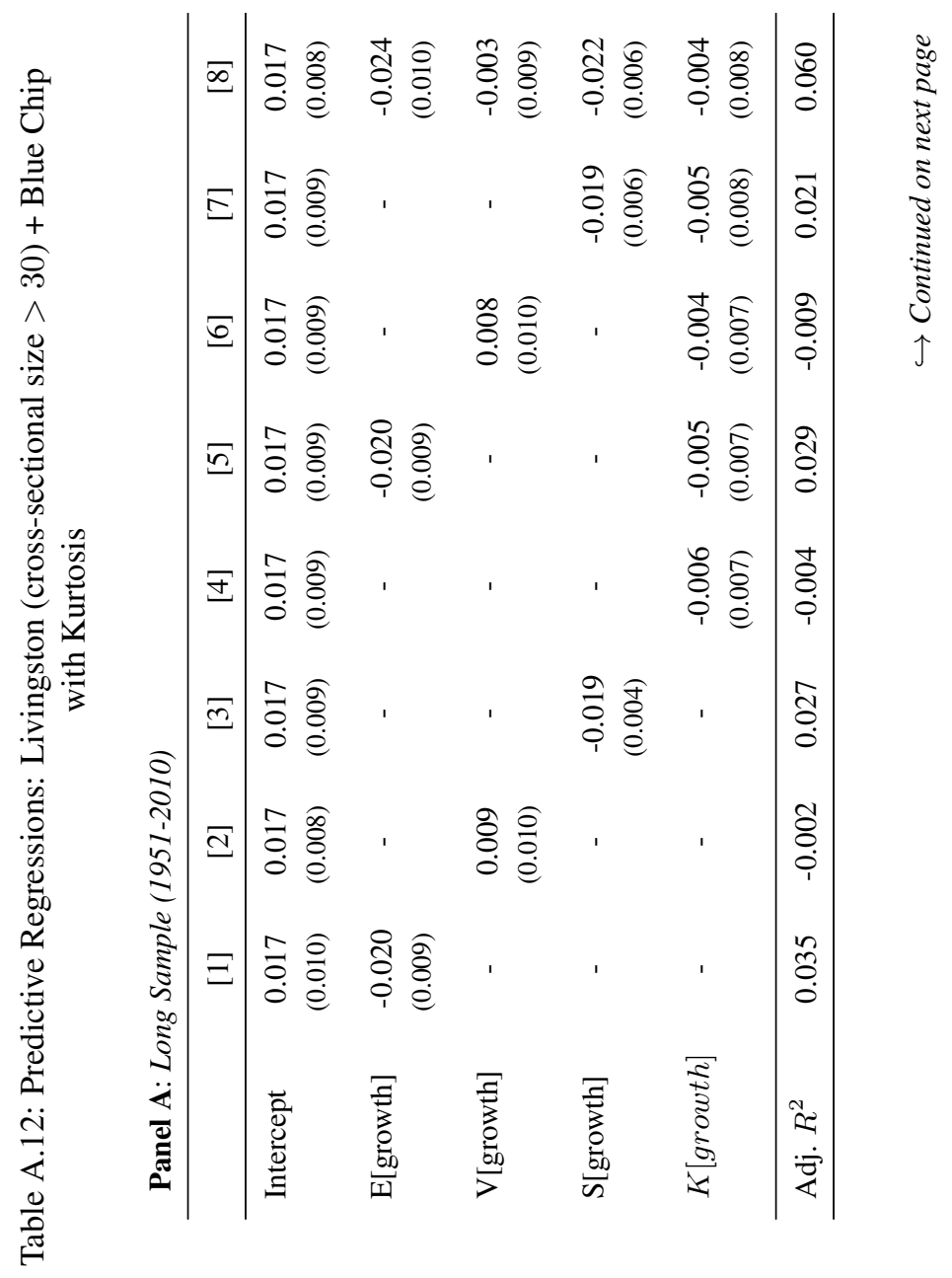




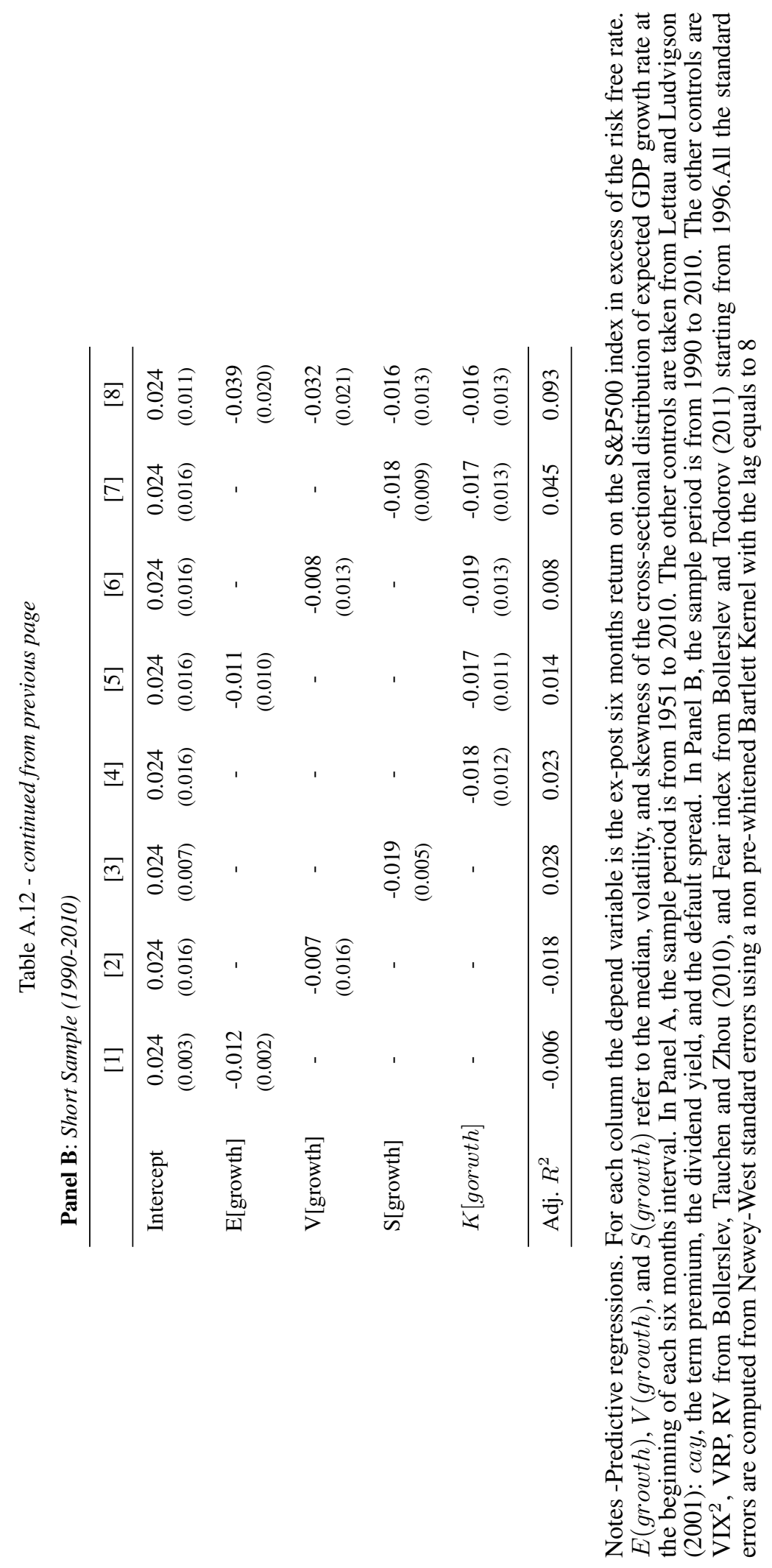




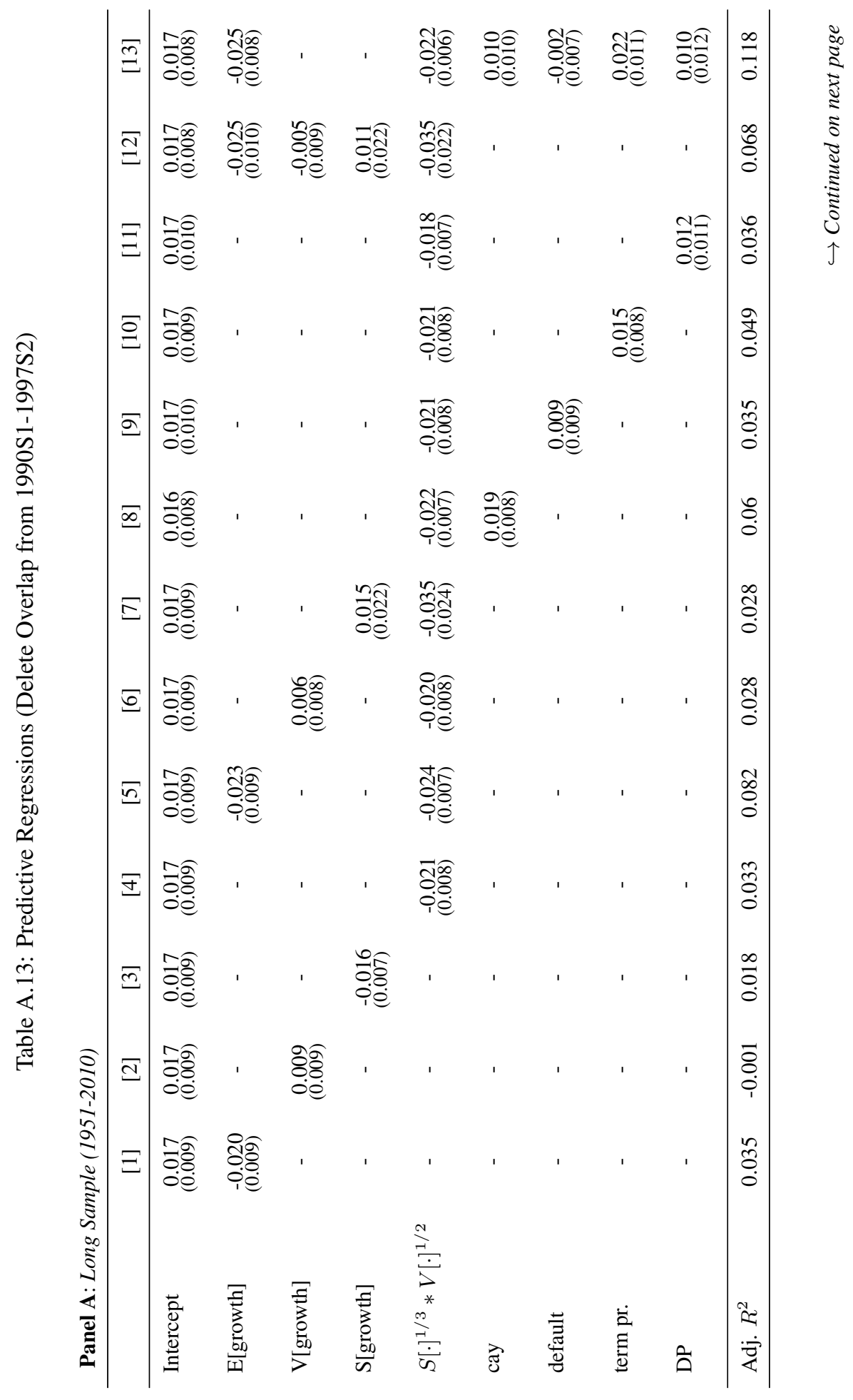




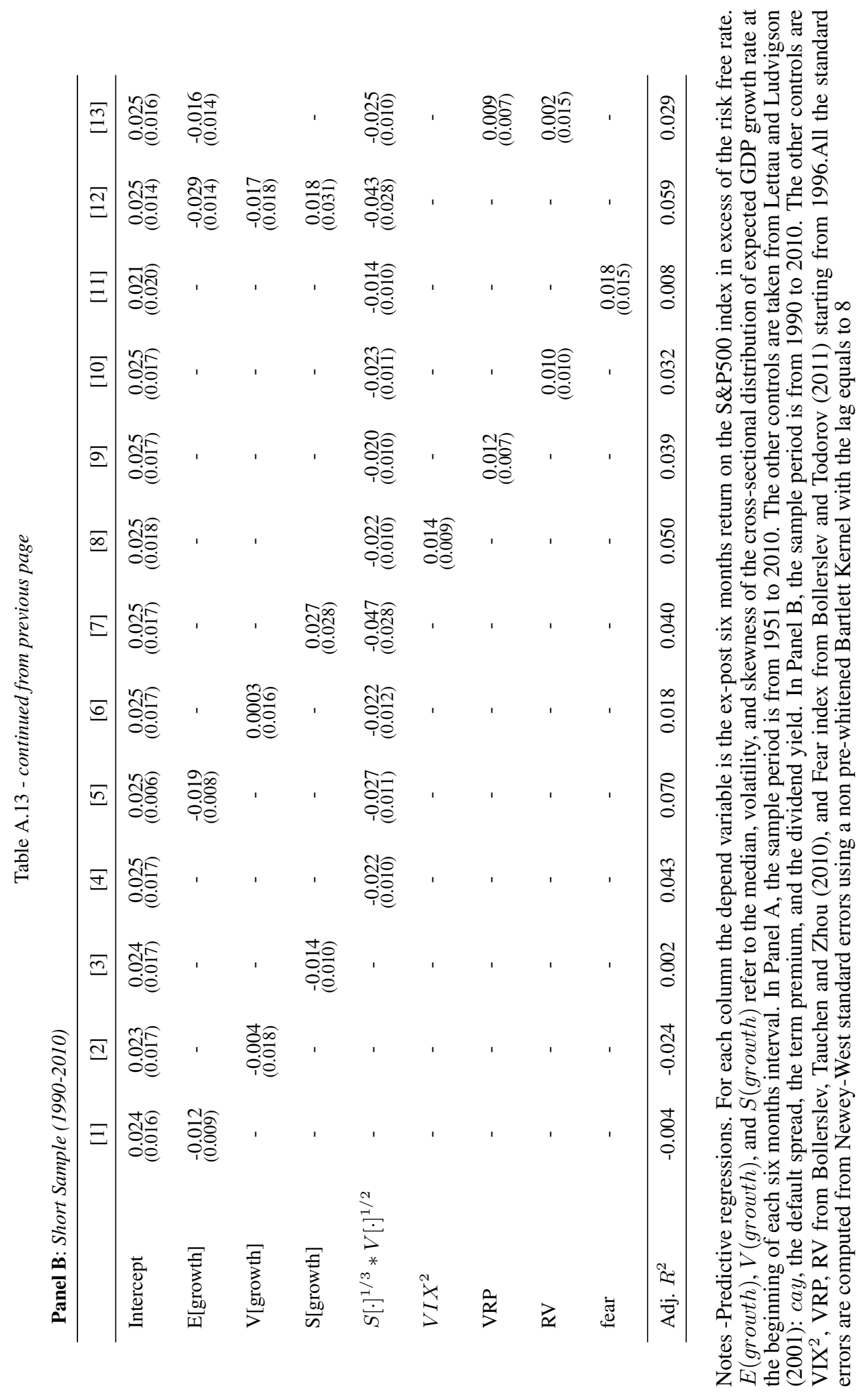


Then:

$$
\begin{aligned}
E \exp \left\{\kappa_{1} z+\kappa_{2} z^{2}\right\}= & \frac{2}{\sqrt{1-2 \kappa_{2} \sigma^{2}}} \cdot \exp \left\{\frac{\frac{\left(\kappa_{1} \sigma^{2}+\mu\right)^{2}}{1-2 \kappa_{2} \sigma^{2}}-\mu^{2}}{2 \sigma^{2}}\right\} \cdot \int_{-\infty}^{+\infty} \frac{1}{\sqrt{2 \pi}} \exp \left\{-\frac{y^{2}}{2}\right\} \\
& \left(\int_{-\infty}^{\frac{\nu}{\sqrt{1-2 \kappa_{2} \sigma^{2}}} y+\frac{\left(\kappa_{1}+2 \mu \kappa_{2}\right) \nu \sigma}{1-2 \kappa_{2} \sigma^{2}}} \frac{1}{\sqrt{2 \pi}} \exp \left\{-\frac{t^{2}}{2}\right\} d t\right) d y
\end{aligned}
$$

Now use the following result from Zacks (1981). If $U$ is a $N(0,1)$ random variable, then

$$
E[\Phi(h U+k)]=\Phi\left(\frac{k}{\sqrt{1+h^{2}}}\right)
$$

for any real $h, k$ and where $\Phi(\cdot)$ denotes the cumulative distribution function of a $N(0,1)$ random variable. This implies that:

$$
\begin{aligned}
E \exp \left\{\kappa_{1} z+\kappa_{2} z^{2}\right\}= & \frac{2}{\sqrt{1-2 \kappa_{2} \sigma^{2}}} \cdot \exp \left\{\frac{1}{2 \sigma^{2}}\left[\frac{\left(\kappa_{1} \sigma^{2}+\mu\right)^{2}}{1-2 \kappa_{2} \sigma^{2}}-\mu^{2}\right]\right\} . \\
& \Phi\left(\frac{\left(\kappa_{1}+2 \mu \kappa_{2}\right) \mu \sigma}{\sqrt{\left(1-2 \kappa_{2} \sigma^{2}\right)\left(1-2 \kappa_{2} \sigma^{2}+\nu^{2}\right)}}\right) .
\end{aligned}
$$

Applying logarithms to both sides concludes the proof.

Proof of Lemma A.1.2. The proof is straightforward:

$$
\begin{aligned}
\log E_{t} \exp \left\{A+B x+C x^{2}\right\} & =\log \int_{-\infty}^{+\infty} \exp \left\{A+B x+C x^{2}\right\} \frac{1}{2 \pi} \exp \left\{-\frac{x^{2}}{2}\right\} d x \\
& =\log \int_{-\infty}^{+\infty} \exp \left\{-\frac{\left(x-\frac{B}{1-2 C}\right)^{2}-\frac{B^{2}+2 A-4 A C}{(1-2 C)^{2}}}{2\left(\frac{1}{1-2 C}\right)}\right\} \\
& =\log \sqrt{\frac{1}{1-2 C}}+\frac{B^{2}+2 A-4 A C}{2(1-2 C)} \\
& =\log \sqrt{\frac{1}{1-2 C}}+\frac{B^{2}}{2(1-2 C)}+A
\end{aligned}
$$


Table A.14: Maximum Percentage Error of Volatility Approximation

\begin{tabular}{lccccccc}
\hline \hline & \multicolumn{7}{c}{$\sqrt{\sigma_{\sigma}} \cdot 10^{-6}$} \\
\cline { 2 - 8 }$\rho_{\sigma}$ & 2 & 2.5 & 3 & 3.5 & 3.8 & 4 & 4.5 \\
\hline 0.8000 & 0.0123 & 0.0194 & 0.0282 & 0.0387 & 0.0458 & 0.0510 & 0.0650 \\
0.8500 & 0.0161 & 0.0254 & 0.0369 & 0.0507 & 0.0602 & 0.0669 & 0.0855 \\
0.9000 & 0.0265 & 0.0419 & 0.0610 & 0.0841 & 0.0998 & 0.1112 & 0.1424 \\
0.9300 & 0.0403 & 0.0639 & 0.0935 & 0.1291 & 0.1536 & 0.1712 & 0.2201 \\
0.9500 & 0.0561 & 0.0892 & 0.1308 & 0.1812 & 0.2159 & 0.2410 & 0.3107 \\
0.9900 & 0.1721 & 0.2774 & 0.4125 & 0.5802 & 0.6979 & 0.7840 & 1.0275 \\
\hline
\end{tabular}

Notes - The table reports the maximum approximation error of the first order Taylor expansion of the time-varying volatility process. In all the calibrations, the unconditional volatility is set to 0.02 .

\section{A.10 Approximations}

\section{A.10.1 Approximation of the volatility process}

. Given the time-varying variance process

$$
\sigma_{t}=\bar{\sigma}\left(1-\rho_{\sigma}\right)+\rho_{\sigma} \sigma_{t-1}+\sigma_{\varepsilon} \varepsilon_{t}^{\sigma}, \text { where } \varepsilon_{t}^{\sigma} \sim N(0,1)
$$

a first order Taylor expansion of the volatility, $\sqrt{\sigma_{t}}$, about $\sqrt{\bar{\sigma}}$ yields

$$
\sqrt{\sigma_{t}} \approx \sqrt{\bar{\sigma}}\left(1-\rho_{\sigma}\right)+\rho_{\sigma} \sqrt{\sigma_{t-1}}+\frac{\sigma_{\varepsilon}}{2 \sqrt{\bar{\sigma}}} \varepsilon_{t}^{\sigma}
$$

Table A.14 documents the accuracy of this approximation for a large number of parameter values including the ones that we use in our calibration. The main finding is that the maximum approximation error is usually less that $1 \%$.

\section{A.10.2 Utility function approximation}

. This approximation is used only for expositional purposes (to highlight the relevance of skewness in our model): for all the solutions both analytical and numerical, we employ the general functional form reported in the dissertation. A third order expansion about $E_{t}\left[U_{t+1}\right]$ yields:

$U_{t} \approx(1-\delta) \log C_{t}+\delta E_{t}\left[U_{t+1}\right]+\delta(1-\gamma) \log \left[1+\frac{(1-\gamma)^{2}}{2} V_{t}\left[U_{t+1}\right]+\frac{(1-\gamma)^{3}}{3 !} E_{t}\left[\left(U_{t+1}-E_{t}\left[U_{t+1}\right]\right)^{3}\right]\right]$ 
We further approximate the logarithmic term as

$$
\frac{(1-\gamma)^{2}}{2} V_{t}\left[U_{t+1}\right]+\frac{(1-\gamma)^{3}}{3 !} E_{t}\left[\left(U_{t+1}-E_{t}\left[U_{t+1}\right]\right)^{3}\right]
$$

to obtain the expression reported in main text and to highlight the role of higher order moments. This approximation is reported only for the purpose of exposition: all the results in the dissertation are derived using the general utility specification in Hansen and Sargent (1995).

\section{A.11 Analytical solution of the case with time-varying volatility and skewness}

We assume that

$$
\sigma_{t}^{x}=\sigma_{t}^{c}=\sigma_{t}=\bar{\sigma}\left(1-\rho_{\sigma}\right)+\rho_{\sigma} \sigma_{t-1}+\sqrt{\sigma_{\varepsilon}} \varepsilon_{t}^{\sigma}, \text { where } \varepsilon_{t}^{\sigma} \sim N(0,1)
$$

Furthermore, we assume that $\phi_{t}=\frac{\nu_{t}}{\sqrt{1+\nu_{t}^{2}}}$ follows an $A R(1)$ process, that is

$$
\phi_{t}=\rho_{\phi} \phi_{t-1}+\sqrt{\sigma_{\phi}} \varepsilon_{t}^{\phi}, \text { where } \varepsilon_{t}^{\phi} \sim N(0,1) .
$$

\section{A.11.1 Utility function}

We can write down the value function for $\sigma$ being time varying as following:

$$
\widetilde{V}_{t}=\delta \theta \log E_{t} \exp \left\{\left[\mu_{c}+\frac{\varphi_{e}^{2} B^{2} \sigma_{t}}{2 \theta}+0.8 \varphi_{e} B \sqrt{\sigma_{t}} \phi_{t+1}+\sqrt{\sigma_{t}} \varepsilon_{t+1}^{c}+\widetilde{V}_{t+1}\right] \cdot \frac{1}{\theta}\right\}
$$

We guess that the solution of this value function is $\widetilde{V}_{t}=V_{0}+V_{\phi, 1} \phi_{t}+V_{\phi, 2} \phi_{t}^{2}+V_{\sigma, 1} \sqrt{\sigma_{t}}+V_{\sigma, 2} \sigma_{t}+$ $V_{\phi, \sigma} \phi_{t} \sqrt{\sigma_{t}}$. By plugging in our guess in the value function above, we get:

$$
\begin{aligned}
\widetilde{V}_{t}= & \delta\left(\mu_{c}+\frac{\varphi_{e}^{2} B^{2} \sigma_{t}}{2 \theta}+V_{0}\right)+\delta \theta \log E_{t} \exp \left\{\frac { 1 } { \theta } \left(0.8 \varphi_{e} B \sqrt{\sigma_{t}} \rho_{\phi} \phi_{t}+0.8 \varphi_{e} B \sqrt{\sigma_{t}} \sqrt{\sigma_{\phi}} \varepsilon_{t+1}^{\phi}\right.\right. \\
& \left.\left.+\sqrt{\sigma_{t}} V_{\phi, 1} \phi_{t+1} \varepsilon_{t+1}^{c}+V_{\phi, 2} \phi_{t+1}^{2}+V_{\sigma, 1} \sqrt{\sigma_{t+1}}+V_{\sigma, 2} \sigma_{t+1}+V_{\phi, \sigma} \phi_{t+1} \sqrt{\sigma_{t+1}}\right)\right\}
\end{aligned}
$$

Using the first order approximation of the of the volatility process described in section A.10.1, we have that

$$
\sqrt{\sigma_{t}} \cdot \phi_{t} \approx\left[\sqrt{\bar{\sigma}}\left(1-\rho_{\sigma}\right)+\rho_{\sigma} \sqrt{\sigma_{t-1}}+\frac{\sqrt{\sigma_{\varepsilon}}}{2 \sqrt{\bar{\sigma}}} \varepsilon_{t}^{\sigma}\right] \cdot\left[\rho_{\phi} \phi_{t-1}+\sqrt{\sigma_{\phi}} \varepsilon_{t}^{\phi}\right]
$$




$$
\begin{aligned}
= & \sqrt{\bar{\sigma}}\left(1-\rho_{\sigma}\right) \rho_{\phi} \phi_{t-1}+\rho_{\sigma} \rho_{\phi} \sqrt{\sigma_{t-1}} \phi_{t-1}+\rho_{\phi} \frac{\sqrt{\sigma_{\varepsilon}}}{2 \sqrt{\bar{\sigma}}} \phi_{t-1} \varepsilon_{t}^{\sigma}+\sqrt{\bar{\sigma}}\left(1-\rho_{\sigma}\right) \sqrt{\sigma_{\phi}} \varepsilon_{t}^{\phi}+ \\
& \rho_{\sigma} \sqrt{\sigma_{\phi}} \sqrt{\sigma_{t-1}} \varepsilon_{t}^{\phi}+\frac{\sqrt{\sigma_{\varepsilon}}}{2 \sqrt{\bar{\sigma}}} \sqrt{\sigma_{\phi}} \varepsilon_{t}^{\sigma} \varepsilon_{t}^{\phi} .
\end{aligned}
$$

It follows that the value function can be written as

$$
\begin{aligned}
\widetilde{V}_{t}= & \delta\left(\mu_{c}+\frac{\varphi_{e}^{2} B^{2}}{2 \theta} \sigma_{t}+V_{0}+0.8 \varphi_{e} B \rho_{\phi} \sqrt{\sigma_{t}} \phi_{t}\right)+ \\
& \delta \theta \log E_{t} \exp \left\{\left[0.8 \varphi_{e} B \sqrt{\sigma_{t}} \sqrt{\sigma_{\phi}} \varepsilon_{t+1}^{\phi}+\sqrt{\sigma_{t}} \varepsilon_{t+1}^{c}+V_{\phi, 1} \rho_{\phi} \phi_{t}\right.\right. \\
& +V_{\phi, 1} \sqrt{\sigma_{\phi}} \varepsilon_{t+1}^{\phi}+V_{\phi, 2} \rho_{\phi}^{2} \phi_{t}^{2}+V_{\phi, 2} \sigma_{\phi}\left(\varepsilon_{t+1}^{\phi}\right)^{2}+2 V_{\phi, 2} \rho_{\phi} \sqrt{\sigma_{\phi}} \phi_{t} \varepsilon_{t+1}^{\phi} \\
& +V_{\sigma, 1} \sqrt{\bar{\sigma}}\left(1-\rho_{\sigma}\right)+V_{\sigma, 1} \rho_{\sigma} \sqrt{\sigma_{t}}+V_{\sigma, 1} \frac{\sqrt{\sigma_{\varepsilon}}}{2 \sqrt{\bar{\sigma}}} \varepsilon_{t+1}^{\sigma} \sqrt{\sigma_{t+1}}+V_{\sigma, 2} \bar{\sigma}\left(1-\rho_{\sigma}\right)+V_{\sigma, 2} \rho_{\sigma} \sigma_{t} \\
& +V_{\sigma, 2} \sqrt{\sigma_{\varepsilon}} \varepsilon_{t+1}^{\sigma}+V_{\phi, \sigma} \sqrt{\bar{\sigma}}\left(1-\rho_{\sigma}\right) \rho_{\phi} \phi_{t}+V_{\phi, \sigma} \rho_{\phi} \rho_{\sigma} \sqrt{\sigma_{t}} \phi_{t}+V_{\phi, \sigma} \rho_{\phi} \frac{\sqrt{\sigma_{\varepsilon}}}{2 \sqrt{\bar{\sigma}}} \phi_{t} \varepsilon_{t+1}^{\sigma} \\
& \left.\left.+V_{\phi, \sigma} \sqrt{\bar{\sigma}}\left(1-\rho_{\sigma}\right) \sqrt{\sigma_{\phi}} \varepsilon_{t+1}^{\phi}+V_{\phi, \sigma} \rho_{\sigma} \sqrt{\sigma_{\phi}} \sqrt{\sigma_{t}} \varepsilon_{t+1}^{\phi}+V_{\phi, \sigma} \frac{\sqrt{\sigma_{\varepsilon}}}{2 \sqrt{\bar{\sigma}}} \phi_{t} \varepsilon_{t+1}^{\sigma} \varepsilon_{t+1}^{\phi}\right] \cdot \frac{1}{\theta}\right\}
\end{aligned}
$$

Using the law of iterated expectations and the result on the expectation of the exponential of a quadratic function of normally distributed random variables discussed in section A.1,

$$
\begin{aligned}
\widetilde{V}_{t}= & \delta\left[V_{0}+\mu_{c}+V_{\sigma, 1} \sqrt{\bar{\sigma}}\left(1-\rho_{\sigma}\right)+V_{\sigma, 2} \bar{\sigma}\left(1-\rho_{\sigma}\right)+\left(V_{\phi, 1} \rho_{\phi}+V_{\phi, \sigma} \sqrt{\bar{\sigma}}\left(1-\rho_{\sigma}\right) \rho_{\phi}\right) \phi_{t}\right. \\
& \left.+V_{\phi, 2} \rho_{\phi}^{2} \phi_{t}^{2}+V_{\sigma, 1} \rho_{\sigma} \sqrt{\sigma_{t}}+\left(\frac{\varphi_{e}^{2} B^{2}}{2 \theta}+V_{\sigma, 2} \rho_{\sigma}\right) \sigma_{t}+\left(0.8 \varphi_{e} B \rho_{\phi}+V_{\phi, \sigma} \rho_{\phi} \rho_{\sigma}\right) \sqrt{\sigma_{t}} \phi_{t}\right] \\
& +\delta \theta \log E_{t}\left[E \left[\operatorname { e x p } \left\{\left[\left(\frac{V_{\sigma, 1}}{2 \sqrt{\bar{\sigma}}}+V_{\sigma, 2}+V_{\phi, \sigma} \frac{\rho_{\phi} \phi_{t}}{2 \sqrt{\bar{\sigma}}}\right)+\sqrt{\sigma_{\varepsilon}} \varepsilon_{t+1}^{\sigma}+\sqrt{\sigma_{t}} \varepsilon_{t+1}^{c}\right.\right.\right.\right. \\
& +\left(0.8 \varphi_{e} B \sqrt{\sigma_{t}}+V_{\phi, 1}+2 V_{\phi, 2} \rho_{\phi} \phi_{t}+V_{\phi, \sigma} \sqrt{\bar{\sigma}}\left(1-\rho_{\sigma}\right)+V_{\phi, \sigma} \rho_{\sigma} \sqrt{\sigma_{t}}\right) \sqrt{\sigma_{\phi}} \varepsilon_{t+1}^{\phi} \\
& \left.\left.\left.\left.+V_{\phi, 2} \sigma_{\phi}\left(\varepsilon_{t+1}^{\phi}\right)^{2}+V_{\phi, \sigma} \frac{\sqrt{\sigma_{\varepsilon}}}{2 \sqrt{\bar{\sigma}}} \sqrt{\sigma_{\phi_{t}}} \varepsilon_{t+1}^{\sigma} \varepsilon_{t+1}^{\phi}\right] \cdot \frac{1}{\theta}\right\} \mid \varepsilon_{t+1}^{\phi}\right]\right] \\
= & \delta\left[V_{0}+\mu_{c}+V_{\sigma, 1} \sqrt{\bar{\sigma}}\left(1-\rho_{\sigma}\right)+V_{\sigma, 2} \bar{\sigma}\left(1-\rho_{\sigma}\right)+\left(V_{\phi, 1} \rho_{\phi}+V_{\phi, \sigma} \sqrt{\bar{\sigma}}\left(1-\rho_{\sigma}\right) \rho_{\phi}\right) \phi_{t}+\right. \\
& \left.V_{\phi, 2} \rho_{\phi}^{2} \phi_{t}^{2}+V_{\sigma, 1} \rho_{\sigma} \sqrt{\sigma_{t}}+\left(\frac{\varphi_{e}^{2} B^{2}}{2 \theta}+V_{\sigma, 2} \rho_{\sigma}\right) \sigma_{t}+\left(0.8 \varphi_{e} B \rho_{\phi}+V_{\phi, \sigma} \rho_{\phi} \rho_{\sigma}\right) \sqrt{\sigma_{t}} \phi_{t}+\frac{\sigma_{t}}{2 \theta}\right] \\
& +\delta \theta \log E_{t} \exp \left\{\left[\left(0.8 \varphi_{e} B \sqrt{\sigma_{t}}+V_{\phi, 1}+2 V_{\phi, 2} \rho_{\phi} \phi_{t}+V_{\phi, \sigma} \sqrt{\bar{\sigma}} \cdot\left(1-\rho_{\sigma}\right)+V_{\phi, \sigma} \rho_{\sigma} \sqrt{\sigma_{t}}\right)\right.\right.
\end{aligned}
$$




$$
\begin{aligned}
& \left.\left.\cdot \sqrt{\sigma_{\phi}} \varepsilon_{t+1}^{\phi}+V_{\phi, 2} \sigma_{\phi}\left(\varepsilon_{t+1}^{\phi}\right)^{2}\right] \cdot \frac{1}{\theta}+\frac{\sigma_{\varepsilon}}{2 \theta^{2}}\left(\frac{V_{\sigma, 1}}{2 \sqrt{\bar{\sigma}}}+V_{\sigma, 2}+V_{\phi, \sigma} \frac{\rho_{\phi} \phi_{t}}{2 \sqrt{\bar{\sigma}}}+\frac{V_{\phi, \sigma}}{2 \sqrt{\bar{\sigma}}} \sqrt{\sigma_{\phi}} \varepsilon_{t+1}^{\phi}\right)^{2}\right\} \\
= & \delta\left[V_{0}+\mu_{c}+V_{\sigma, 1} \sqrt{\bar{\sigma}}\left(1-\rho_{\sigma}\right)+V_{\sigma, 2} \bar{\sigma}\left(1-\rho_{\sigma}\right)+\left(V_{\phi, 1} \rho_{\phi}+V_{\phi, \sigma} \sqrt{\bar{\sigma}}\left(1-\rho_{\sigma}\right) \rho_{\phi}\right) \phi_{t}\right. \\
& \left.+V_{\phi, 2} \rho_{\phi}^{2} \phi_{t}^{2}+V_{\sigma, 1} \rho_{\sigma} \sqrt{\sigma_{t}}+\left(\frac{\varphi_{e}^{2} B^{2}}{2 \theta}+V_{\sigma, 2} \rho_{\sigma}\right) \sigma_{t}+\left(0.8 \varphi_{e} B \rho_{\phi}+V_{\phi, \sigma} \rho_{\phi} \rho_{\sigma}\right) \sqrt{\sigma_{t}} \phi_{t}+\frac{\sigma_{t}}{2 \theta}\right] \\
& +\delta \theta \log E_{t} \exp \left\{\frac { 1 } { \theta } \cdot \left[\left(0.8 \varphi_{e} B+V_{\phi, \sigma} \rho_{\sigma}\right) \sqrt{\sigma_{t}}+V_{\phi, 1}+2 V_{\phi, 2} \rho_{\phi} \phi_{t}+V_{\phi, \sigma} \sqrt{\bar{\sigma}}\left(1-\rho_{\sigma}\right)\right.\right. \\
& \left.\left.+\frac{V_{\sigma, 1} V_{\phi, \sigma}}{2 \bar{\sigma}} \frac{\sigma_{\varepsilon}}{2 \theta} \frac{V_{\sigma, 2} V_{\phi, \sigma}}{\sqrt{\bar{\sigma}}} \frac{\sigma_{\varepsilon}}{2 \theta}+\frac{V_{\phi, \sigma}^{2} \rho_{\phi} \phi_{t}}{2 \bar{\sigma}} \frac{\sigma_{\varepsilon}}{2 \theta}\right] \sqrt{\sigma_{\phi}} \varepsilon_{t+1}^{\phi}+\frac{1}{\theta} \cdot\left(V_{\phi, 2}+\frac{V_{\phi, \sigma}^{2}}{4 \bar{\sigma}} \frac{\sigma_{\varepsilon}}{2 \theta}\right) \sigma_{\phi}\left(\varepsilon_{t+1}^{\phi}\right)^{2}\right\} .
\end{aligned}
$$

Then the value function boils down to

$$
\begin{aligned}
& \widetilde{V}_{t}=\delta\left[\left(V_{0}+\mu_{c}+V_{\sigma, 1} \sqrt{\bar{\sigma}}\left(1-\rho_{\sigma}\right)+V_{\sigma, 2} \bar{\sigma}\left(1-\rho_{\sigma}\right)\right)+\frac{\sigma_{\varepsilon}}{2 \theta}\left(\frac{V_{\sigma, 1}^{2}}{4 \bar{\sigma}}+V_{\sigma, 2}^{2}+\frac{V_{\phi, \sigma}^{2} \rho_{\phi}^{2} \phi_{t}^{2}}{4 \bar{\sigma}}\right.\right. \\
& \left.+\frac{V_{\sigma, 1} V_{\sigma, 2}}{\sqrt{\bar{\sigma}}}+\frac{V_{\sigma, 1} V_{\phi, \sigma} \rho_{\phi} \phi_{t}}{2 \bar{\sigma}}+\frac{V_{\sigma, 2} V_{\phi, 2} \rho_{\phi} \phi_{t}}{\sqrt{\bar{\sigma}}}\right)+\phi_{t}\left(V_{\phi, 1} \rho_{\phi}+V_{\phi, \sigma} \sqrt{\bar{\sigma}}\left(1-\rho_{\sigma}\right) \rho_{\phi}\right) \\
& \left.+\phi_{t}^{2}\left(V_{\phi, 2} \rho_{\phi}^{2}\right)+\sqrt{\sigma_{t}}\left(V_{\sigma, 1} \rho_{\sigma}\right)+\sigma_{t}\left(\frac{\varphi_{e}^{2} B^{2}}{2 \theta}+V_{\sigma, 2} \rho_{\sigma}\right)+\sqrt{\sigma_{t}} \phi_{t}\left(0.8 \varphi_{e} B \rho_{\phi}+V_{\phi, \sigma} \rho_{\phi} \rho_{\sigma}\right)+\frac{\sigma_{t}}{2 \theta}\right] \\
& -\frac{\theta \delta}{2} \log \left(1-\frac{2}{\theta}\left(V_{\phi, 2}+\frac{V_{\phi, \sigma}^{2}}{4 \bar{\sigma}}\right) \sigma_{\phi}\right)+\frac{\delta \theta}{2 \delta^{2}}\left[1-\frac{2}{\theta}\left(V_{\phi, 2}+\frac{V_{\phi, \sigma}^{2}}{4 \bar{\sigma}}\right) \sigma_{\phi}\right]^{-1} \cdot \sigma_{\phi} \cdot \\
& {\left[\left(0.8 \varphi_{e} B+V_{\phi, \sigma} \rho_{\sigma}\right)^{2} \sigma_{t}+V_{\phi, 1}^{2}+4 V_{\phi, 2}^{2} \rho_{\phi}^{2} \phi_{t}^{2}+V_{\phi, \sigma}^{2} \bar{\sigma}\left(1-\rho_{\sigma}\right)^{2}+\frac{V_{\sigma, 1}^{2} V_{\phi, \sigma}^{2}}{4 \bar{\sigma}^{2}} \frac{\sigma_{\varepsilon}^{2}}{4 \theta^{2}}+\left(\frac{V_{\sigma, 2} V_{\phi, \sigma}}{\sqrt{\bar{\sigma}}} \frac{\sigma_{\varepsilon}}{2 \theta}\right)^{2}\right.} \\
& +\left(\frac{V_{\phi, \sigma}^{2} \rho_{\phi} \phi_{t}}{2 \bar{\sigma}} \frac{\sigma_{\varepsilon}}{2 \theta}\right)^{2}+4\left(0.8 \varphi_{e} B \rho_{\phi}+V_{\phi, \sigma} \rho_{\phi}\right) V_{\phi, 2} \rho_{\phi} \phi_{t} \sqrt{\sigma_{t}}+2 V_{\phi, 1}\left(0.8 \varphi_{e} B \rho_{\phi}+V_{\phi, \sigma} \rho_{\phi}\right) \sqrt{\sigma_{t}}+ \\
& 2 V_{\phi, \sigma} \sqrt{\bar{\sigma}}\left(1-\rho_{\sigma}\right)\left(0.8 \varphi_{e} B \rho_{\phi}+V_{\phi, \sigma} \rho_{\phi}\right) \sqrt{\sigma_{t}}+\left(0.8 \varphi_{e} B \rho_{\phi}+V_{\phi, \sigma} \rho_{\phi}\right) \\
& \left(\frac{V_{\sigma, 1} V_{\phi, \sigma}}{2 \bar{\sigma}}+\frac{V_{\sigma, 2} V_{\phi, \sigma}}{\sqrt{\bar{\sigma}}}+\frac{V_{\phi, \sigma}^{2} \rho_{\phi} \phi_{t}}{2 \bar{\sigma}}\right) \frac{\sigma_{\varepsilon}}{\theta} \sqrt{\sigma_{t}}+\phi_{t} \cdot 4 V_{\phi, 2} \rho_{\phi} \cdot\left(V_{\phi, 1}+V_{\phi, \sigma} \sqrt{\bar{\sigma}}\left(1-\rho_{\sigma}\right)+\right. \\
& \left.\frac{\sigma_{\varepsilon}}{2 \theta}\left(\frac{V_{\sigma, 1} V_{\phi, \sigma}}{2 \bar{\sigma}}+\frac{V_{\sigma, 2} V_{\phi, \sigma}}{\sqrt{\bar{\sigma}}}+\frac{V_{\phi, \sigma}^{2} \rho_{\phi} \phi_{t}}{2 \bar{\sigma}}\right)\right)+2 V_{\phi, 1}\left(V_{\phi, \sigma} \sqrt{\bar{\sigma}}\left(1-\rho_{\sigma}\right)+\right. \\
& \left.\frac{\sigma_{\varepsilon}}{2 \theta}\left(\frac{V_{\sigma, 1} V_{\phi, \sigma}}{2 \bar{\sigma}}+\frac{V_{\sigma, 2} V_{\phi, \sigma}}{\sqrt{\bar{\sigma}}}+\frac{V_{\phi, \sigma}^{2} \rho_{\phi} \phi_{t}}{2 \bar{\sigma}}\right)\right) 2 V_{\phi, \sigma} \sqrt{\bar{\sigma}}\left(1-\rho_{\sigma}\right) \cdot \frac{\sigma_{\varepsilon}}{2 \theta}\left(\frac{V_{\sigma, 1} V_{\phi, \sigma}}{2 \bar{\sigma}}+\frac{V_{\sigma, 2} V_{\phi, \sigma}}{\sqrt{\bar{\sigma}}}+\frac{V_{\phi, \sigma}^{2} \rho_{\phi} \phi_{t}}{2 \bar{\sigma}}\right) \\
& \left.+\frac{\sigma_{\varepsilon}}{\theta} \frac{V_{\sigma, 1} V_{\phi, \sigma}}{2 \bar{\sigma}} \frac{\sigma_{\varepsilon}}{2 \theta}\left(\frac{V_{\sigma, 2} V_{\phi, \sigma}}{\sqrt{\bar{\sigma}}}+\frac{V_{\phi, \sigma}^{2} \rho_{\phi} \phi_{t}}{2 \bar{\sigma}}\right)+2\left(\frac{\sigma_{\varepsilon}}{2 \theta}\right)^{2} \frac{V_{\sigma, 2} V_{\phi, \sigma}}{\sqrt{\bar{\sigma}}} \cdot \frac{V_{\phi, \sigma}^{2} \rho_{\phi} \phi_{t}}{2 \bar{\sigma}}\right]
\end{aligned}
$$

Matching coefficient yields the following system of equations:

$$
\begin{aligned}
V_{0}= & \delta\left[V_{0}+\mu_{c}+V_{\sigma, 1} \sqrt{\bar{\sigma}}\left(1-\rho_{\sigma}\right)+\frac{\sigma_{\varepsilon}}{2 \theta}\left(\frac{V_{\sigma, 1}}{4 \bar{\sigma}}+V_{\sigma, 2}^{2}+\frac{V_{\sigma, 1} V_{\sigma, 2}}{\sqrt{\bar{\sigma}}}\right)\right] \\
& -\frac{\delta \theta}{2} \log \left(1-\frac{2}{\theta}\left(V_{\phi, 2}+\frac{\sigma_{\varepsilon}}{2 \theta} \cdot \frac{V_{\phi, \sigma}^{2}}{4 \bar{\sigma}}\right) \sigma_{\phi}\right)+\frac{\delta}{2 \theta}\left[1-\frac{2}{\theta}\left(V_{\phi, 2}+\frac{\sigma_{\varepsilon}}{2 \theta} \cdot \frac{V_{\phi, \sigma}^{2}}{4 \bar{\sigma}}\right) \sigma_{\phi}\right]^{-1} \cdot \sigma_{\phi} .
\end{aligned}
$$




$$
\begin{aligned}
& \left\{V_{\phi, 1}^{2}+V_{\phi, \sigma}^{2} \bar{\sigma}\left(1-\rho_{\sigma}\right)^{2}+\left(\frac{V_{\sigma, 1} V_{\phi, \sigma}}{2 \bar{\sigma}} \cdot \frac{\sigma_{\varepsilon}}{2 \theta}\right)^{2}+\left(\frac{V_{\sigma, 2} V_{\phi, \sigma}}{\sqrt{\bar{\sigma}}} \cdot \frac{\sigma_{\varepsilon}}{2 \theta}\right)^{2}+\frac{\sigma_{\varepsilon}}{2 \theta}\left(\frac{V_{\sigma, 1} V_{\phi, \sigma}}{2 \bar{\sigma}}+\frac{V_{\sigma, 2} V_{\phi, \sigma}}{\sqrt{\bar{\sigma}}}\right)+\right. \\
& 2 V_{\phi, 1} V_{\phi, \sigma} \sqrt{\bar{\sigma}}\left(1-\rho_{\sigma}\right)+\frac{V_{\phi, 1} \sigma_{\varepsilon}}{\theta}\left(\frac{V_{\sigma, 1} V_{\phi, \sigma}}{2 \bar{\sigma}}+\frac{V_{\sigma, 2} V_{\phi, \sigma}}{\sqrt{\bar{\sigma}}}\right)+\frac{V_{\sigma, 1} V_{\phi, \sigma}^{2} \sigma_{\varepsilon}^{2}}{4 \bar{\sigma} \theta^{2}} \cdot \frac{V_{\sigma, 2}}{\sqrt{\bar{\sigma}}} \\
& \left.+2 V_{\phi, \sigma} \sqrt{\bar{\sigma}}\left(1-\rho_{\sigma}\right) \frac{\sigma_{\varepsilon}}{2 \theta} \cdot\left(\frac{V_{\sigma, 1} V_{\phi, \sigma}}{2 \bar{\sigma}}+\frac{V_{\sigma, 2} V_{\phi, \sigma}}{\sqrt{\bar{\sigma}}}\right)\right\} \\
& V_{\phi, 1}=\delta\left[\frac{V_{\sigma, 1} V_{\phi, \sigma} \rho_{\phi}}{2 \bar{\sigma}} \cdot \frac{\sigma_{\varepsilon}}{2 \theta}+\frac{V_{\sigma, 2} V_{\phi, 2} \rho_{\phi}}{\sqrt{\bar{\sigma}}} \cdot \frac{\sigma_{\varepsilon}}{2 \theta}+V_{\phi, 1} \rho_{\phi}+V_{\phi, \sigma} \sqrt{\bar{\sigma}}\left(1-\rho_{\sigma}\right) \rho_{\phi}\right]+\frac{\delta}{2 \theta} \cdot \sigma_{\phi} \\
& \cdot\left[1-\frac{2}{\theta}\left(V_{\phi, 2}+\frac{\sigma_{\varepsilon}}{2 \theta} \cdot \frac{V_{\phi, \sigma}^{2}}{4 \bar{\sigma}}\right) \sigma_{\phi}\right]^{-1} \cdot\left\{4 V_{\phi, 2} \rho_{\phi}\left[V_{\phi, 1}+V_{\phi, \sigma} \sqrt{\bar{\sigma}}\left(1-\rho_{\sigma}\right)+\frac{\sigma_{\varepsilon}}{2 \theta}\left(\frac{V_{\sigma, 1} V_{\phi, \sigma}}{2 \bar{\sigma}}+\frac{V_{\sigma, 2} V_{\phi, \sigma}}{\sqrt{\bar{\sigma}}}\right)\right]\right. \\
& \left.+V_{\phi, 1} \cdot \frac{\sigma_{\varepsilon}}{2 \theta} \cdot \frac{V_{\phi, \sigma}^{2} \rho_{\phi}}{\bar{\sigma}}+\frac{\sigma_{\varepsilon}}{2 \theta} \cdot \frac{V_{\phi, \sigma}^{2} \rho_{\phi}}{\bar{\sigma}}\left[V_{\phi, \sigma} \sqrt{\bar{\sigma}}\left(1-\rho_{\sigma}\right)+\frac{\sigma_{\varepsilon}}{2 \theta} \cdot\left(\frac{V_{\sigma, 1} V_{\phi, \sigma}}{2 \bar{\sigma}}+\frac{V_{\sigma, 2} V_{\phi, \sigma}}{\sqrt{\bar{\sigma}}}\right)\right]\right\} \\
& V_{\phi, 2}=\delta\left(\frac{\sigma_{\varepsilon}}{2 \theta} \cdot \frac{V_{\phi, \sigma}^{2} \rho_{\phi}^{2}}{4 \bar{\sigma}}+V_{\phi, 2} \rho_{\phi}^{2}\right)+\frac{\delta}{2 \theta}\left[1-\frac{2}{\theta}\left(V_{\phi, 2}+\frac{\sigma_{\varepsilon}}{2 \theta} \cdot \frac{V_{\phi, \sigma}^{2}}{4 \bar{\sigma}}\right) \sigma_{\phi}\right]^{-1} \cdot \sigma_{\phi} \rho_{\phi}^{2} \cdot\left(2 V_{\phi, 2}+\frac{\sigma_{\varepsilon} V_{\phi, \sigma}^{2}}{4 \theta \bar{\sigma}}\right)^{2} \\
& V_{\sigma, 1}=\delta V_{\sigma, 1} \rho_{\sigma}+\frac{\delta}{2 \theta}\left[1-\frac{2}{\theta}\left(V_{\phi, 2}+\frac{\sigma_{\varepsilon}}{2 \theta} \cdot \frac{V_{\phi, \sigma}^{2}}{4 \bar{\sigma}}\right) \sigma_{\phi}\right]^{-1} \cdot \sigma_{\phi} \cdot\left(0.8 \varphi_{e} B+V_{\phi, \sigma} \rho_{\sigma}\right) \cdot \\
& {\left[2 V_{\phi, 1}+2 V_{\phi, \sigma} \sqrt{\bar{\sigma}}\left(1-\rho_{\sigma}\right)+\left(\frac{V_{\sigma, 1} V_{\phi, \sigma}}{2 \bar{\sigma}}+\frac{V_{\sigma, 2} V_{\phi, \sigma}}{\sqrt{\bar{\sigma}}}\right) \cdot \frac{\sigma_{\varepsilon}}{\theta}\right]} \\
& V_{\sigma, 2}=\delta\left[\frac{\varphi_{e}^{2} B^{2}}{2 \theta}+V_{\sigma, 2} \rho_{\sigma}+\frac{1}{2 \theta}\right]+\frac{\delta}{2 \theta}\left[1-\frac{2}{\theta}\left(V_{\phi, 2}+\frac{\sigma_{\varepsilon}}{2 \theta} \cdot \frac{V_{\phi, \sigma}^{2}}{4 \bar{\sigma}}\right) \sigma_{\phi}\right]^{-1} \cdot \sigma_{\phi} \cdot\left(0.8 \varphi_{e} B+V_{\phi, \sigma} \rho_{\sigma}\right)^{2} \\
& V_{\phi, \sigma}=\delta \rho_{\phi}\left(0.8 \varphi_{e} B+V_{\phi, \sigma} \rho_{\sigma}\right)+\frac{\delta}{2 \theta}\left[1-\frac{2}{\theta}\left(V_{\phi, 2}+\frac{\sigma_{\varepsilon}}{2 \theta} \cdot \frac{V_{\phi, \sigma}^{2}}{4 \bar{\sigma}}\right) \sigma_{\phi}\right]^{-1} \cdot \sigma_{\phi} \rho_{\phi} \\
& \cdot\left[\left(0.8 \varphi_{e} B+V_{\phi, \sigma} \rho_{\phi}\right) \cdot\left(4 V_{\phi, 2}+\frac{V_{\phi, \sigma}^{2}}{\bar{\sigma}} \cdot \frac{\sigma_{\varepsilon}}{2 \theta}\right)\right]
\end{aligned}
$$

We take a first order Taylor expansion about the parameter values of the model without time-varying volatility. That is, we expand the system of equations in (A.17) about

$$
\begin{aligned}
& \sigma_{\varepsilon}=0, \quad \rho_{\sigma}=0, \quad V_{\phi, 1}=\frac{0.8 B \delta^{2} \varphi_{e} \rho_{\phi}^{2} \sqrt{\bar{\sigma}}}{1-\delta \rho_{\phi}}, \quad V_{\phi, 2}=0, \\
& V_{\sigma, 1}=\frac{0.8^{2} B^{2} \delta^{2} \varphi_{e}^{2} \rho_{\phi} \sqrt{\bar{\sigma}} \sigma_{\phi}}{\theta}, \quad V_{\sigma, 2}=\frac{\delta}{2 \theta}\left(1+B^{2} \delta \varphi_{e}^{2}+0.8^{2} \sigma_{\phi} B^{2} \delta \varphi_{e}^{2}\right), \quad V_{\phi, \sigma}=0.8 B \delta \varphi_{e} \rho_{\phi} .
\end{aligned}
$$

The resulting system is as follows

$$
\begin{aligned}
0.8 B \delta \varphi_{e} \rho_{\phi}-V_{\phi, \sigma}+0.8 B \delta^{2} \varphi_{e} \rho_{\phi}^{2} \rho_{\sigma}+\frac{8 B V_{\phi, 2} \delta \varphi_{e} \rho_{\phi} \sigma_{\phi}}{5 \theta} & =0 \\
V_{\phi, 1}\left(\delta \rho_{\phi}-1\right)+\delta \rho_{\phi} \sqrt{\bar{\sigma}} V_{\phi, \sigma}+\frac{2}{\theta} V_{\phi, 2} \frac{\delta^{2} \rho_{\phi}^{2}}{1-\delta \rho_{\phi}} \sigma_{\phi}\left(0.8 B \varphi_{e} \sqrt{\bar{\sigma}}\right)-0.8 B \delta^{2} \varphi_{e} \rho_{\phi}^{2} \rho_{\sigma} \sqrt{\bar{\sigma}} & =0 \\
V_{\phi, 2}\left(\delta \rho_{\phi}^{2}-1\right) & =0 \\
\frac{0.8^{2} B^{2} \delta^{2} \varphi_{e}^{2} \rho_{\phi} \sqrt{\bar{\sigma}} \sigma_{\phi}\left(\delta \rho_{\sigma}-\rho_{\sigma}+2 \delta \rho_{\phi} \rho_{\sigma}+1\right)}{\theta\left(1-\delta \rho_{\phi}\right)}-V_{\sigma, 1} & =0
\end{aligned}
$$




$$
\delta\left(\rho_{\sigma} \delta+1\right)\left(\frac{1+B^{2} \varphi_{e}^{2}+0.8^{2} B^{2} \varphi_{e}^{2} \sigma_{\phi}}{2 \theta}\right)+\frac{0.8^{2} B^{2} \delta \varphi_{e}^{2} \sigma_{\phi}}{\theta}\left(\rho_{\phi} \rho_{\sigma}+\frac{V_{\phi, 2}}{\theta}\right)-V_{\sigma, 2}=0
$$

The solution of the system is

$$
\begin{aligned}
V_{\phi, 1} & =\frac{0.8 B \delta^{2} \varphi_{e} \rho_{\phi}^{2} \sqrt{\bar{\sigma}}\left(\delta \rho_{\phi} \rho_{\sigma}-\rho_{\sigma}+1\right)}{1-\delta \rho_{\phi}}>0 \\
V_{\phi, 2} & =0 \\
V_{\sigma, 1} & =\frac{0.8^{2} B^{2} \delta^{2} \varphi_{e}^{2} \rho_{\phi} \sqrt{\bar{\sigma}} \sigma_{\phi}\left(\delta \rho_{\sigma}-\rho_{\sigma}+2 \delta \rho_{\phi} \rho_{\sigma}+1\right)}{\theta\left(1-\delta \rho_{\phi}\right)}<0 \\
V_{\sigma, 2} & =\frac{\delta}{2 \theta}\left[\left(1+\delta \rho_{\sigma}\right)\left(1+B^{2} \varphi_{e}^{2}\left(1+0.8^{2} \sigma_{\phi}\right)\right)+2 \cdot 0.8^{2} B^{2} \varphi_{e}^{2} \sigma_{\phi} \delta \rho_{\phi} \rho_{\sigma}\right]<0 \\
V_{\phi, \sigma} & =0.8 B \delta \varphi_{e} \rho_{\phi}\left(1+\delta \rho_{\phi} \rho_{\sigma}\right)>0
\end{aligned}
$$

The signs of the coefficients follow immediately from the stationarity of the state variables and from $\theta<0$.

\section{A.11.2 Stochastic discount factor}

The stochastic discount factor is the marginal rate of intertemporal substitution:

$$
\begin{aligned}
m_{t+1} & =\log \delta-\Delta c_{t+1}+\frac{U_{t}+1}{\theta}-\log E_{t} \exp \left\{\frac{U_{t+1}}{\theta}\right\} \\
& =\log \delta-\left(1-\frac{1}{\theta}\right) \Delta c_{t+1}+\frac{V_{t+1}}{\theta}-\log E_{t} \exp \left\{\frac{V_{t+1}+\Delta c_{t+1}}{\theta}\right\} \\
& =\log \delta-\left(1-\frac{1}{\delta}\right) \Delta c_{t+1}+\frac{V_{t+1}}{\theta}-\frac{V_{t}}{\delta \theta}
\end{aligned}
$$

The innovations to the stochastic discount factor can be written as

$$
m_{t+1}-E_{t} m_{t+1}=-\left(1-\frac{1}{\theta}\right)\left(\Delta c_{t+1}-E_{t} \Delta c_{t+1}\right)+\frac{1}{\theta}\left(V_{t+1}-E_{t} V_{t+1}\right)
$$

Since

$$
\begin{aligned}
V_{t}-E_{t-1} V_{t}= & B \varphi_{e} \sqrt{\sigma_{t-1}} \varepsilon_{t}^{x}+\left[V_{\sigma, 1} \frac{1}{2 \sqrt{\bar{\sigma}}}+V_{\sigma, 2}+V_{\phi, \sigma} \rho_{\phi} \frac{1}{2 \sqrt{\bar{\sigma}}} \phi_{t-1}\right] \varepsilon_{t}^{\sigma}+\left[V_{\phi, 1}+2 V_{\phi, 2} \rho_{\phi} \phi_{t-1}\right. \\
& \left.+V_{\phi, \sigma} \sqrt{\bar{\sigma}}\left(1-\rho_{\sigma}\right)+\rho_{\sigma} \sqrt{\sigma_{t-1}} V_{\phi, \sigma}\right] \sqrt{\sigma_{\phi}} \varepsilon_{t}^{\phi}+V_{\phi, \sigma} \frac{\sigma_{\varepsilon}}{2 \sqrt{\bar{\sigma}}} \sqrt{\varepsilon_{t}^{\sigma}} \varepsilon_{t}^{\phi}+V_{\phi, 2} \sigma_{\phi}\left(\varepsilon_{t}^{\phi}\right)^{2},
\end{aligned}
$$


the equation for the innovations to the SDF is:

$$
\begin{aligned}
m_{t+1}-E_{t} m_{t+1}= & -\left(1-\frac{1}{\theta}\right) \sigma_{t} \varepsilon_{t+1}^{c}+\frac{1}{\theta} B \varphi_{e} \sqrt{\sigma_{t}} \varepsilon_{t+1}^{x}+\frac{1}{\theta}\left(V_{\sigma, 1} \frac{1}{2 \sqrt{\bar{\sigma}}}+V_{\sigma, 2}+V_{\phi, \sigma} \rho_{\phi} \frac{1}{2 \sqrt{\bar{\sigma}}} \phi_{t}\right) \varepsilon_{t+1}^{\sigma} \\
& +\frac{1}{\theta}\left[V_{\phi, 1}+2 V_{\phi, 2} \rho_{\phi} \phi_{t}+V_{\phi, \sigma} \sqrt{\bar{\sigma}}\left(1-\rho_{\sigma}\right)+\rho_{\sigma} \sqrt{\sigma_{t}} V_{\phi, \sigma}\right] \sqrt{\sigma_{\phi}} \varepsilon_{t+1}^{\phi} \\
& +\frac{1}{\theta} V_{\phi, \sigma} \frac{\sqrt{\sigma_{\varepsilon}}}{2 \sqrt{\bar{\sigma}}} \sqrt{\sigma_{\phi}} \varepsilon_{t+1}^{\sigma} \varepsilon_{t+1}^{\phi}+V_{\phi, 2} \sigma_{\phi}\left(\varepsilon_{t+1}^{\phi}\right)^{2}
\end{aligned}
$$

Since $V_{t+1}$ can be written as

$$
\begin{aligned}
V_{t+1}= & \widetilde{V}_{t+1}+B X_{t+1} \\
= & V_{0}+V_{\phi, 1} \phi_{t+1}+V_{\phi, 2} \phi_{t+1}^{2}+V_{\sigma, 1} \sqrt{\sigma_{t+1}}+V_{\sigma, 2} \sigma_{t+1}+V_{\phi, \sigma} \phi_{t+1} \sqrt{\sigma_{t+1}}+B X_{t+1} \\
= & V_{0}+V_{\phi, 1} \rho_{\phi} \phi_{t}+V_{\phi, 1} \rho_{\phi, 1} \sqrt{\sigma_{\phi}} \varepsilon_{t+1}^{\phi}+V_{\phi, 2} \rho_{\phi}^{2} \phi_{t}^{2}+V_{\phi, 2} \sigma_{\phi}\left(\varepsilon_{t+1}^{\phi}\right)^{2}+2 V_{\phi, 2} \rho_{\phi} \sqrt{\sigma_{\phi}} \phi_{t} \varepsilon_{t+1}^{\phi} \\
& +V_{\sigma, 1} \sqrt{\bar{\sigma}}\left(1-\rho_{\sigma}\right)+V_{\sigma, 1} \rho_{\sigma} \sqrt{\sigma_{t}}+V_{\sigma, 1} \frac{\sqrt{\sigma_{\varepsilon}}}{2 \sqrt{\bar{\sigma}}} \varepsilon_{t+1}^{\sigma}+V_{\phi, \sigma} \sqrt{\bar{\sigma}}\left(1-\rho_{\sigma}\right) \rho_{\phi} \phi_{t}+V_{\sigma, 2} \bar{\sigma}\left(1-\rho_{\sigma}\right)+V_{\sigma, 2} \rho_{\sigma} \sigma_{t} \\
& +V_{\sigma, 2} \sqrt{\sigma_{\varepsilon}} \varepsilon_{t+1}^{\sigma}+V_{\phi, \sigma} \rho_{\sigma} \rho_{\phi} \sqrt{\sigma_{t}} \phi_{t}+V_{\phi, \sigma} \rho_{\phi} \frac{\sqrt{\sigma_{\varepsilon}}}{2 \sqrt{\bar{\sigma}}} \phi_{t} \varepsilon_{t+1}^{\sigma}+V_{\phi, \sigma} \sqrt{\bar{\sigma}}\left(1-\rho_{\sigma}\right) \sqrt{\sigma_{\phi}} \varepsilon_{t+1}^{\phi} \\
& +V_{\phi, \sigma} \rho_{\sigma} \sqrt{\sigma_{\phi}} \sqrt{\sigma_{t}} \varepsilon_{t+1}^{\phi}+V_{\phi, \sigma} \frac{\sqrt{\sigma_{\varepsilon}}}{2 \sqrt{\bar{\sigma}}} \sqrt{\sigma_{\phi}} \varepsilon_{t+1}^{\sigma} \varepsilon_{t+1}^{\phi}+B \rho_{x} x_{t}+B \varphi_{e} \sqrt{\sigma_{t}} \varepsilon_{t+1}^{x}
\end{aligned}
$$

it follows that the SDF is:

$$
\begin{aligned}
& m_{t+1}=\log \delta-\left(1-\frac{1}{\theta}\right)\left(\mu_{c}+x_{t}+\sqrt{\sigma_{t}} \varepsilon_{t+1}^{c}\right)+\frac{1}{\theta}\left\{\left(V_{\phi, 1}+2 V_{\phi, 2} \rho_{\phi} \phi_{t}+V_{\phi, \sigma} \sqrt{\bar{\sigma}}\left(1-\rho_{\sigma}\right)+V_{\phi, \sigma} \rho_{\sigma} \sqrt{\sigma_{t}}\right) .\right. \\
& \left.\sqrt{\sigma_{\phi}} \varepsilon_{t+1}^{\phi}+V_{\phi, \sigma} \frac{\sqrt{\sigma_{\varepsilon}}}{2 \sqrt{\bar{\sigma}}} \sqrt{\sigma_{\phi}} \varepsilon_{t+1}^{\sigma} \varepsilon_{t+1}^{\phi}+V_{\phi, 2} \sigma_{\phi}\left(\varepsilon_{t+1}^{\phi}\right)^{2}+\left(\frac{V_{\sigma, 1}}{2 \sqrt{\bar{\sigma}}}+V_{\sigma, 2}+\frac{V_{\phi, \sigma} \rho_{\phi} \phi_{t}}{2 \sqrt{\bar{\sigma}}}\right) \sqrt{\sigma_{\varepsilon}} \varepsilon_{t+1}^{\sigma}\right\} \\
& +\frac{1}{\theta}\left(B \rho_{x} x_{t}+B \varphi_{e} \sqrt{\sigma_{t}} \varepsilon_{t+1}^{x}\right)-\log E_{t} \exp \left\{\frac { 1 } { \theta } \left[\left(V_{\phi, 1}+2 V_{\phi, 2} \rho_{\phi} \phi_{t}+V_{\phi, \sigma} \sqrt{\bar{\sigma}}\left(1-\rho_{\sigma}\right)+V_{\phi, \sigma} \rho_{\sigma} \sqrt{\sigma_{t}}\right) .\right.\right. \\
& \sqrt{\sigma_{\phi}} \varepsilon_{t+1}^{\phi}+V_{\phi, \sigma} \frac{\sqrt{\sigma_{\varepsilon}}}{2 \sqrt{\bar{\sigma}}} \sqrt{\sigma_{\phi}} \varepsilon_{t+1}^{\sigma} \varepsilon_{t+1}^{\phi} V_{\phi, 2} \sigma_{\phi}\left(\varepsilon_{t+1}^{\phi}\right)^{2}+\left(\frac{V_{\sigma, 1}}{2 \sqrt{\bar{\sigma}}}+V_{\sigma, 2}+\frac{V_{\phi, \sigma} \rho_{\phi} \phi_{t}}{2 \sqrt{\bar{\sigma}}}\right) \sqrt{\sigma_{\varepsilon}} \varepsilon_{t+1}^{\sigma} \\
& \left.\left.+\mu_{c}+x_{t}+\sqrt{\sigma_{t}^{c}} \varepsilon_{t+1}+B \rho_{x} x_{t}+B \varphi_{e} \sqrt{\sigma_{t}} \varepsilon_{t+1}^{x}\right]\right\}
\end{aligned}
$$

By defining the following coefficients:

$$
\begin{aligned}
m_{t+1}= & m_{0}-x_{t}-m_{\phi, 1} \phi_{t}-m_{\phi, 2} \phi_{t}^{2}-m_{\sigma, 1} \sqrt{\sigma_{t}}-m_{\sigma, 2} \sigma_{t}-m_{\phi, \sigma} \phi_{t} \sqrt{\sigma_{t}} \\
& -\sqrt{z_{c}} \varepsilon_{t+1}^{c}-\sqrt{z_{x}} \varepsilon_{t+1}^{x}-\sqrt{z_{\phi, 1}} \varepsilon_{t+1}^{\phi}-\sqrt{z_{\sigma}} \varepsilon_{t+1}^{\sigma}-\sqrt{z_{\phi, \sigma}} \varepsilon_{t+1}^{\phi} \varepsilon_{t+1}^{\sigma}-\sqrt{z_{\phi, 2}}\left(\varepsilon_{t+1}^{\phi}\right)^{2}
\end{aligned}
$$


where

$$
\begin{aligned}
& m_{0}=\log \delta-\mu_{c}+\frac{1}{2} \log (1-2 c)-\frac{\sigma_{\varepsilon}}{2 \theta}\left(\frac{V_{\sigma, 1}^{2}}{4 \bar{\sigma}}+V_{\sigma, 2}^{2}+\frac{V_{\sigma, 1} V_{\sigma, 2}}{\sqrt{\bar{\sigma}}}\right)-\frac{1}{2 \theta^{2}}(1-2 c)^{-1} \sigma_{\phi} . \\
& \left\{V_{\phi, 1}^{2}+V_{\phi, \sigma}^{2} \bar{\sigma}\left(1-\rho_{\sigma}\right)^{2}+\left(\frac{V_{\sigma, 1} V_{\phi, \sigma}}{2 \bar{\sigma}} \frac{\sigma_{\varepsilon}}{2 \theta}\right)^{2}\left(\frac{V_{\sigma, 2} V_{\phi, \sigma}}{\sqrt{\bar{\sigma}}} \frac{\sigma_{\varepsilon}}{2 \theta}\right)^{2}+2 V_{\phi, 1} V_{\phi, \sigma} \sqrt{\bar{\sigma}}\left(1-\rho_{\sigma}\right)\right. \\
& \left.+\frac{\sigma_{\varepsilon}}{\theta}\left[V_{\phi, 1}+V_{\phi, \sigma} \sqrt{\bar{\sigma}}\left(1-\rho_{\sigma}\right)\right] \cdot\left(\frac{V_{\sigma, 1} V_{\phi, \sigma}}{2 \bar{\sigma}}+\frac{V_{\sigma, 2} V_{\phi, \sigma}}{\sqrt{\bar{\sigma}}}\right)+\frac{V_{\sigma, 1} V_{\phi, \sigma}}{2 \bar{\sigma}} \frac{V_{\sigma, 2} V_{\phi, \sigma}}{\sqrt{\bar{\sigma}}} \frac{\sigma_{\varepsilon}^{2}}{2 \theta^{2}}\right\} \\
& m_{\phi, 1}=\frac{1}{2 \theta^{2}}(1-2 c)^{-1} \sigma_{\phi} \cdot\left\{4 V_{\phi, 2} \rho_{\phi}\left[\left(V_{\phi, 1}+V_{\phi, \sigma} \sqrt{\bar{\sigma}}\left(1-\rho_{\sigma}\right)\right)+\frac{\sigma_{\varepsilon}}{2 \theta}\left(\frac{V_{\sigma, 1} V_{\phi, \sigma}}{2 \bar{\sigma}}+\frac{V_{\sigma, 2} V_{\phi, \sigma}}{\sqrt{\bar{\sigma}}}\right)\right]\right. \\
& \left.+\left(\frac{\sigma_{\varepsilon}}{2 \theta}\right)^{2} \frac{V_{\sigma, 2} V_{\phi, \sigma}^{3} \rho_{\phi}}{(\sqrt{\bar{\sigma}})^{3}}+\frac{\sigma_{\varepsilon}}{2 \theta} \frac{V_{\phi, \sigma}^{2} V_{\phi, 1} \rho_{\phi}}{\bar{\sigma}}+\frac{\sqrt{\bar{\sigma}}\left(1-\rho_{\sigma}\right) \sigma_{\varepsilon} V_{\phi, \sigma}^{3} \rho_{\phi}}{2 \theta \bar{\sigma}}+\frac{V_{\sigma, 1} V_{\phi, \sigma}^{3} \sigma_{\varepsilon}^{2} \rho_{\phi}}{8 \bar{\sigma}^{2} \theta^{2}}\right\} \\
& +\left(\frac{V_{\sigma, 1}}{2 \bar{\sigma}}+\frac{V_{\sigma, 2}}{\sqrt{\bar{\sigma}}}\right) \frac{\sigma_{\varepsilon} V_{\phi, \sigma} \rho_{\phi}}{2 \theta^{2}} \\
& m_{\phi, 2}=\frac{\sigma_{\varepsilon} V_{\phi, \sigma}^{2} \rho_{\phi}^{2}}{8 \bar{\sigma} \theta^{2}}+\frac{1}{2 \theta^{2}}(1-2 c)^{-1} \sigma_{\phi}\left\{4 V_{\phi, 2}^{2} \rho_{\phi}^{2}+\left(\frac{V_{\phi, \sigma}^{2} \rho_{\phi} \sigma_{\varepsilon}}{4 \bar{\sigma} \theta}\right)^{2}+\frac{V_{\phi, 2} \rho_{\phi}^{2} \sigma_{\varepsilon} V_{\phi, \sigma}^{2}}{\theta \bar{\sigma}}\right\} \\
& m_{\sigma, 1}=\frac{1}{2 \theta^{2}}(1-2 c)^{-1} \sigma_{\phi}\left\{2 V_{\phi, 1}+2 V_{\phi, \sigma} \sqrt{\bar{\sigma}}\left(1-\rho_{\sigma}\right)+\left(\frac{V_{\sigma, 1} V_{\phi, \sigma}}{2 \bar{\sigma}}+\frac{V_{\sigma, 2} V_{\phi, \sigma}}{\sqrt{\bar{\sigma}}}\right) \frac{\sigma_{\varepsilon}}{\theta}\right\} . \\
& \left(0.8 \varphi_{e} B+V_{\phi, \sigma} \rho_{\sigma}\right) \\
& m_{\sigma, 2}=\frac{1}{2 \theta^{2}}+\frac{1}{2}\left(\frac{B \varphi_{e}}{\theta}\right)^{2}+\frac{1}{2 \theta^{2}}(1-2 c)^{-1} \sigma_{\phi}\left(0.8 \varphi_{e} B+V_{\phi, \sigma} \rho_{\sigma}\right)^{2} \\
& m_{\phi, \sigma}=\frac{1}{2 \theta^{2}}(1-2 c)^{-1} \sigma_{\phi}\left\{4 V_{\phi, 2} \rho_{\phi}+\left(\frac{V_{\phi, \sigma}^{2} \rho_{\phi} \sigma_{\varepsilon}}{2 \bar{\sigma} \theta}\right)\right\}\left(0.8 \varphi_{e} B+V_{\phi, \sigma} \rho_{\sigma}\right)+\frac{0.8 B \varphi_{e} \rho_{\phi}}{\theta} \\
& \sqrt{z_{c}}=\left(1-\frac{1}{\theta}\right) \sqrt{\sigma_{t}} \\
& \sqrt{z_{x}}=-\frac{B \varphi_{e}}{\theta} \sqrt{\sigma_{t}} \\
& \sqrt{z_{\phi, 1}}=-\frac{\sqrt{\sigma_{\phi}}}{\theta}\left(V_{\phi, 1}+2 V_{\phi, 2} \rho_{\phi} \phi_{t}+V_{\phi, \sigma} \sqrt{\bar{\sigma}}\left(1-\rho_{\sigma}\right)+V_{\phi, \sigma} \rho_{\sigma} \sqrt{\sigma_{t}}\right) \\
& \sqrt{z_{\phi, 2}}=-\frac{V_{\phi, 2} \sigma_{\phi}}{\theta} \\
& \sqrt{z_{\sigma}}=-\frac{\sqrt{\sigma_{\varepsilon}}}{\theta}\left(\frac{V_{\sigma, 1}}{2 \sqrt{\bar{\sigma}}}+V_{\sigma, 2}+\frac{V_{\phi, \sigma} \rho_{\phi} \phi_{t}}{2 \sqrt{\bar{\sigma}}}\right) \\
& \sqrt{z_{\phi, \sigma}}=-\frac{V_{\phi, \sigma} \sqrt{\sigma_{\phi}} \sqrt{\sigma_{\varepsilon}}}{2 \sqrt{\bar{\sigma}} \theta} \\
& c=\left(V_{\phi, 2}+\frac{\sigma_{\varepsilon}}{2 \theta} \frac{V_{\phi, \sigma}^{2}}{4 \bar{\sigma}}\right) \frac{\sigma_{\phi}}{\theta}
\end{aligned}
$$


the SDF can be written in compact form as

$$
\begin{aligned}
m_{t+1}= & m_{0}-x_{t}-m_{\phi, 1} \phi_{t}-m_{\phi, 2} \phi_{t}^{2}-m_{\sigma, 1} \sqrt{\sigma_{t}}-m_{\sigma, 2} \sigma_{t}-m_{\phi, \sigma} \phi_{t} \sqrt{\sigma_{t}} \\
& -\sqrt{z_{c}} \varepsilon_{t+1}^{c}-\sqrt{z_{x}} \varepsilon_{t+1}^{x}-\sqrt{z_{\phi, 1}} \varepsilon_{t+1}^{\phi}-\sqrt{z_{\sigma}} \varepsilon_{t+1}^{\sigma}-\sqrt{z_{\phi, \sigma}} \varepsilon_{t+1}^{\phi} \varepsilon_{t+1}^{\sigma}-\sqrt{z_{\phi, 2}}\left(\varepsilon_{t+1}^{\phi}\right)^{2} .
\end{aligned}
$$

\section{A.11.3 Equity returns}

We compute returns on a claim to the following dividend process:

$$
\Delta d_{t}=\lambda_{1} \mu_{c}+\lambda\left(x_{t-1}+\sqrt{\sigma_{t-1}} \varepsilon_{t}^{c}\right)+\sqrt{\sigma_{d}} \sqrt{\sigma_{t-1}} \varepsilon_{t}^{d} \text {, where } \varepsilon_{t}^{d} \sim N(0,1) .
$$

Using the Euler equation and the Campbell and Shiller (1988) approximation we obtain:

$$
v_{d, t}=\log E_{t} \exp \left\{m_{t+1}+\lambda_{1} \mu_{c}+\lambda\left(x_{t}+\sqrt{\sigma_{t}} \varepsilon_{t+1}^{c}\right)+\sqrt{\sigma_{d}} \sqrt{\sigma_{t}} \varepsilon_{t+1}^{d}+K_{0}+K_{1} v_{d, t+1}\right\} .
$$

We guess that $v_{d, t}=v_{0}+v_{x} x_{t}+v_{\phi, 1} \phi_{t}+v_{\phi, 2} \phi_{t}^{2}+v_{\sigma, 1} \sqrt{\sigma_{t}}+v_{\sigma, 2} \sigma_{t}+v_{\phi, \sigma} \phi_{t} \sqrt{\sigma_{t}}$.

Then,

$$
\begin{aligned}
v_{d, t}= & \log E_{t} \exp \left\{m_{0}-x_{t}-m_{\phi, 1} \phi_{t}-m_{\phi, 2} \phi_{t}^{2}-m_{\sigma, 1} \sqrt{\sigma_{t}}-m_{\sigma, 2} \sigma_{t}-m_{\phi, \sigma} \phi_{t} \sqrt{\sigma_{t}}\right. \\
& -\sqrt{z_{c}} \varepsilon_{t+1}^{c}-\sqrt{z_{x}} \varepsilon_{t+1}^{x}-\sqrt{z_{\phi, 1}} \varepsilon_{t+1}^{\phi}-\sqrt{z_{\sigma}} \varepsilon_{t+1}^{\sigma}-\sqrt{z_{\phi, \sigma}} \varepsilon_{t+1}^{\phi} \varepsilon_{t+1}^{\sigma}-\sqrt{z_{\phi, 2}}\left(\varepsilon_{t+1}^{\phi}\right)^{2} \\
& +\lambda_{1} \mu_{c}+\lambda\left(x_{t}+\sqrt{\sigma_{t}} \varepsilon_{t+1}^{c}\right)+\sqrt{\sigma_{d}} \sqrt{\sigma_{t}} \varepsilon_{t+1}^{d}+K_{0}+K_{1}\left(v_{0}+v_{x} x_{t+1}+v_{\phi, 1} \phi_{t+1}+v_{\phi, 2} \phi_{t+1}^{2}\right. \\
& \left.\left.+v_{\sigma, 1} \sqrt{\sigma_{t+1}}+v_{\sigma, 2} \sigma_{t+1}+v_{\phi, \sigma} \phi_{t+1} \sqrt{\sigma_{t+1}}\right)\right\}
\end{aligned}
$$

Therefore, we can rewrite the $v_{d, t}$ function as follows:

$$
\begin{aligned}
v_{d, t}= & m_{0}+\lambda_{1} \mu_{c}+K_{0}+K_{1} v_{0}+K_{1} v_{\sigma, 1} \sqrt{\bar{\sigma}}\left(1-\rho_{\sigma}\right)+K_{1} v_{\sigma, 2} \bar{\sigma}\left(1-\rho_{\sigma}\right) \\
& +\left[K_{1} v_{x, 1} \rho_{x}-(1-\lambda)\right] x_{t}+\left[K_{1} v_{\phi, 1} \rho_{\phi}+K_{1} v_{\phi, \sigma} \rho_{\phi} \sqrt{\bar{\sigma}}\left(1-\rho_{\sigma}\right)-m_{\phi, 1}\right] \phi_{t}+\left(K_{1} v_{\phi, 2} \rho_{\phi}^{2}-m_{\phi, 2}\right) \phi_{t}^{2} \\
& +\left(K_{1} v_{\sigma, 1} \rho_{\sigma}-m_{\sigma, 1}\right) \sqrt{\sigma_{t}}+\left(K_{1} v_{\sigma, 2} \rho_{\sigma}-m_{\sigma, 2}\right) \sigma_{t}+\left(K_{1} v_{\phi, \sigma} \rho_{\phi} \rho_{\sigma}-m_{\phi, \sigma}\right) \phi_{t} \sqrt{\sigma_{t}} \\
& +\log E_{t} \exp \left\{-\left(\sqrt{z_{c}}-\lambda \sqrt{\sigma_{t}}\right) \varepsilon_{t+1}^{c}+\sqrt{\sigma_{d}} \sqrt{\sigma_{t}} \varepsilon_{t+1}^{d}-\left(\sqrt{z_{x}}-K_{1} v_{x, 1} \varphi_{e} \sqrt{\sigma_{t}}\right) \varepsilon_{t+1}^{x}\right. \\
& -\left[\sqrt{z_{\phi, 1}}-K_{1} v_{\phi, 1} \sqrt{\sigma_{\phi}}-K_{1} v_{\phi, 2} 2 \rho_{\phi} \sqrt{\sigma_{\phi}} \phi_{t}-K_{1} v_{\phi, \sigma} \sqrt{\sigma_{\phi}} \sqrt{\bar{\sigma}}\left(1-\rho_{\sigma}\right)-K_{1} v_{\phi, \sigma} \sqrt{\sigma_{\phi}} \rho_{\sigma} \sqrt{\sigma_{t}}\right] \varepsilon_{t+1}^{\phi} \\
& -\left(\sqrt{z_{\sigma}}-K_{1} v_{\sigma, 1} \frac{\sqrt{\sigma_{\varepsilon}}}{2 \sqrt{\bar{\sigma}}}-K_{1} v_{\sigma, 2} \sigma_{\varepsilon}-K_{1} v_{\phi, \sigma} \rho_{\phi} \phi_{t} \frac{\sqrt{\sigma_{\varepsilon}}}{2 \sqrt{\bar{\sigma}}}\right) \varepsilon_{t+1}^{\sigma}
\end{aligned}
$$




$$
\left.-\left(\sqrt{z_{\phi, \sigma}}-K_{1} v_{\phi, \sigma} \sqrt{\sigma_{\phi}} \frac{\sqrt{\sigma_{\varepsilon}}}{2 \sqrt{\bar{\sigma}}}\right) \varepsilon_{t+1}^{\phi} \varepsilon_{t+1}^{\sigma}-\left(\sqrt{z_{\phi, 2}}-K_{1} v_{\phi, 2} \sigma_{\phi}\right)\left(\varepsilon_{t+1}^{\phi}\right)^{2}\right\}
$$

We then apply the law of iterated expectation and the result on the expectation of the exponential of a quadratic function of normally distributed random variables discussed in section A.1, the $V_{d, t}$ can be expressed as follows:

$$
\begin{aligned}
v_{d, t}= & {\left[m_{0}+\lambda_{1} \mu_{c}+K_{0}+K_{1} v_{0}+K_{1} v_{\sigma, 1} \sqrt{\bar{\sigma}}\left(1-\rho_{\sigma}\right)+K_{1} v_{\sigma, 2} \bar{\sigma}\left(1-\rho_{\sigma}\right)\right]+\left[K_{1} v_{x, 1} \rho_{x}-(1-\lambda)\right] \cdot x_{t} } \\
& +\left[K_{1} v_{\phi, 1} \rho_{\phi}+K_{1} v_{\phi, \sigma} \rho_{\phi} \sqrt{\bar{\sigma}}\left(1-\rho_{\sigma}\right)-m_{\phi, 1}\right] \cdot \phi_{t}+\left(K_{1} v_{\phi, 2} \rho_{\phi}^{2}-m_{\phi, 2}\right) \cdot \phi_{t}^{2} \\
& +\left(K_{1} v_{\sigma, 1} \rho_{\sigma}-m_{\sigma, 1}\right) \cdot \sqrt{\sigma_{t}}+\left(K_{1} v_{\sigma, 2} \rho_{\sigma}-m_{\sigma, 2}\right) \cdot \sigma_{t}+\left(K_{1} v_{\phi, \sigma} \rho_{\phi} \rho_{\sigma}-m_{\phi, \sigma}\right) \cdot \phi_{t} \sqrt{\sigma_{t}} \\
& +\frac{1}{2}\left(\sqrt{z_{c}}-\lambda \sqrt{\sigma_{t}}\right)^{2}+\frac{\sigma_{d} \sigma_{t}}{2}+\frac{1}{2}\left(\sqrt{z_{\sigma}}-K_{1} v_{\sigma, 1} \frac{\sqrt{\sigma_{\varepsilon}}}{2 \sqrt{\bar{\sigma}}}-K_{1} v_{\sigma, 2} \sigma_{\varepsilon}-K_{1} v_{\phi, \sigma} \rho_{\phi} \frac{\sqrt{\sigma_{\varepsilon}}}{2 \sqrt{\bar{\sigma}}} \phi_{t}\right)^{2} \\
& +\log E_{t} \exp \left\{\left[\frac{1}{2}\left(\sqrt{z_{\phi, \sigma}}-K_{1} v_{\phi, \sigma} \sqrt{\sigma_{\phi}} \frac{\sqrt{\sigma_{\varepsilon}}}{2 \sqrt{\bar{\sigma}}}\right)^{2}-\sqrt{z_{\phi, 2}}+K_{1} v_{\phi, 2} \sigma_{\phi}\right] \cdot\left(\varepsilon_{t+1}^{\phi}\right)^{2}\right. \\
& +\left[\frac{\sqrt{\sigma_{\phi}} \sigma_{\varepsilon}}{2 \sqrt{\bar{\sigma}}}\left(\frac{V_{\phi, \sigma}}{\theta}+K_{1} v_{\phi, \sigma}\right) \cdot\left(\frac{1}{2 \sqrt{\bar{\sigma}}}\left(\frac{V_{\sigma, 1}}{\theta}+K_{1} v_{\sigma, 1}\right)+\left(\frac{V_{\sigma, 2}}{\theta}+v_{\sigma, 2}\right)\right)\right. \\
& +\sqrt{\sigma_{\phi}}\left(\frac{V_{\phi, 1}}{\theta}+K_{1} v_{\phi, 1}\right)+\sqrt{\bar{\sigma}}\left(1-\rho_{\sigma}\right) \sqrt{\sigma_{\phi}}\left(\frac{V_{\phi, \sigma}}{\theta}++K_{1} v_{\phi, \sigma}\right) \\
& +\phi_{t} \cdot\left(\frac{\sqrt{\sigma_{\phi}} \rho_{\phi} \sigma_{\varepsilon}}{4 \bar{\sigma}}\left(\frac{V_{\phi, \sigma}}{\theta}+K_{1} v_{\phi, \sigma}\right)^{2}+2 \sqrt{\sigma_{\phi}} \rho_{\phi}\left(\frac{V_{\phi, 2}}{\theta}+K_{1} v_{\phi, 2}\right)\right) \\
& \left.\left.+\sqrt{\sigma_{t}} \cdot\left(\frac{0.8 B \varphi_{e} \sqrt{\sigma_{\phi}}}{\theta}+0.8 K_{1} v_{x} \varphi_{e} \sqrt{\sigma_{\phi}}+\sqrt{\sigma_{\phi}} \rho_{\sigma}\left(\frac{V_{\phi, \sigma}}{\theta}+K_{1} v_{\phi, \sigma}\right)\right)\right] \cdot \varepsilon_{t+1}^{\phi}\right\}
\end{aligned}
$$

By using again the result on the expectation of the exponential of a quadratic function of normally distributed random variables, we define the following terms:

$$
C=\frac{1}{2}\left(\sqrt{z_{\phi, \sigma}}-K_{1} v_{\phi, \sigma} \sqrt{\sigma_{\phi}} \frac{\sqrt{\sigma_{\varepsilon}}}{2 \sqrt{\bar{\sigma}}}\right)^{2}-\sqrt{z_{\phi, 2}}+K_{1} v_{\phi, 2} \sigma_{\phi}, \quad B=B_{0}+B_{\phi} \phi_{t}+B_{\sigma} \sqrt{\sigma_{t}}
$$

where

$$
B_{0}=\frac{\sqrt{\sigma_{\phi}} \sigma_{\varepsilon}}{2 \sqrt{\bar{\sigma}}}\left(\frac{V_{\phi, \sigma}}{\theta}+K_{1} v_{\phi, \sigma}\right) \cdot\left[\frac{1}{2 \sqrt{\bar{\sigma}}}\left(\frac{V_{\sigma, 1}}{\theta}+K_{1} v_{\sigma, 1}\right)+\left(\frac{V_{\sigma, 2}}{\theta}+K_{1} v_{\sigma, 2}\right)\right]
$$




$$
\begin{aligned}
& +\sqrt{\sigma_{\phi}}\left(\frac{V_{\phi, 1}}{\theta}+K_{1} v_{\phi, 1}\right)+\sqrt{\bar{\sigma}}\left(1-\rho_{\sigma}\right) \sqrt{\sigma_{\phi}}\left(\frac{V_{\phi, \sigma}}{\theta}++K_{1} v_{\phi, \sigma}\right) . \\
B_{\phi}= & \frac{\sqrt{\sigma_{\phi}} \rho_{\phi} \sigma_{\varepsilon}}{4 \bar{\sigma}}\left(\frac{V_{\phi, \sigma}}{\theta}+K_{1} v_{\phi, \sigma}\right)^{2}+2 \sqrt{\sigma_{\phi}} \rho_{\phi}\left(\frac{V_{\phi, 2}}{\theta}+K_{1} v_{\phi, 2}\right) \\
B_{\sigma}= & \frac{0.8 B \varphi_{e} \sqrt{\sigma_{\phi}}}{\theta}+0.8 K_{1} v_{x} \varphi_{e} \sqrt{\sigma_{\phi}}+\sqrt{\sigma_{\phi}} \rho_{\sigma}\left(\frac{V_{\phi, \sigma}}{\theta}+K_{1} v_{\phi, \sigma}\right) .
\end{aligned}
$$

Matching coefficients:

$$
\begin{aligned}
v_{0}= & \frac{1}{1-K_{1}}\left\{m_{0}+\lambda_{1} \mu_{c}+K_{0}+K_{1} v_{\sigma, 1} \sqrt{\bar{\sigma}}\left(1-\rho_{\sigma}\right)+K_{1} v_{\sigma, 2} \bar{\sigma}\left(1-\rho_{\sigma}\right)+\right. \\
& \left.+\frac{\sigma_{\varepsilon}}{2}\left[\frac{1}{2 \sqrt{\bar{\sigma}}}\left(\frac{V_{\sigma, 1}}{\theta}+K_{1} v_{\sigma, 1}\right)+\left(\frac{V_{\sigma, 2}}{\theta}+K_{1} v_{\sigma, 2}\right)\right]^{2}-\frac{1}{2} \log (1-2 C)+\frac{B_{0}^{2}}{2(1-2 C)}\right\} \\
v_{x}= & \frac{\lambda-1}{1-K_{1} \rho_{x}} \\
v_{\phi, 1}= & \frac{1}{1-K_{1} \rho_{\phi}}\left\{-m_{\phi, 1}+K_{1} v_{\phi, \sigma} \rho_{\phi} \sqrt{\bar{\sigma}}\left(1-\rho_{\sigma}\right)+\frac{\sigma_{\varepsilon} \rho_{\phi}}{2 \sqrt{\bar{\sigma}}} \cdot\left(\frac{V_{\phi, \sigma}}{\theta}+K_{1} v_{\phi, \sigma}\right)\right. \\
& \left.\cdot\left[\frac{1}{2 \sqrt{\bar{\sigma}}}\left(\frac{V_{\sigma, 1}}{\theta}+K_{1} v_{\sigma, 1}\right)+\left(\frac{V_{\sigma, 2}}{\theta}+K_{1} v_{\sigma, 2}\right)\right]+\frac{B_{0} B_{\phi}}{1-2 C}\right\} \\
v_{\phi, 2}= & \frac{1}{1-K_{1} \rho_{\phi}^{2}}\left[-m_{\phi, 2}+\frac{\sigma_{\varepsilon} \rho_{\phi}^{2}}{8 \bar{\sigma}}\left(\frac{V_{\phi, \sigma}}{\theta}+K_{1} v_{\phi, \sigma}\right)^{2}+\frac{B_{\phi}^{2}}{2(1-2 C)}\right] \\
v_{\sigma, 1}= & \frac{1}{1-K_{1} \rho_{\sigma}}\left(-m_{\sigma, 1}+\frac{B_{0} B_{\sigma}}{1-2 C}\right) \\
v_{\sigma, 2}= & \frac{1}{1-K_{1} \rho_{\sigma}}\left[-m_{\sigma, 2}+\frac{\sigma_{d}}{2}+\frac{1}{2}\left(1-\frac{1}{\theta}-\lambda\right)^{2}+\frac{1}{2}\left(\frac{B \varphi_{e}}{\theta}+K_{1} v_{x} \varphi_{e}\right)^{2}+\frac{B_{\sigma}^{2}}{2(1-2 C)}\right] \\
v_{\phi, \sigma}= & \frac{1}{1-K_{1} \rho_{\phi} \rho_{\sigma}}\left[-m_{\phi, \sigma}+0.8 \rho_{\phi}\left(\frac{B \varphi_{e}}{\theta}+K_{1} v_{x} \varphi_{e}\right)+\frac{B_{\phi} B_{\sigma}}{(1-2 C)}\right]
\end{aligned}
$$

Therefore the log-equity returns are equal to:

$$
\begin{aligned}
\log R_{t+1}= & \log \left(1+\exp \left\{V_{d, t+1}\right\}\right)-V_{d, t}+\Delta d_{t+1} \\
\approx & K_{0}+K_{1}\left(v_{0}+v_{x} x_{t+1}+v_{\phi, 1} \phi_{t+1}+v_{\phi, 2} \phi_{t+1}^{2}+v_{\sigma, 1} \sqrt{\sigma_{t+1}}+v_{\sigma, 2} \sigma_{t+1}^{2}+v_{\phi, \sigma} \phi_{t+1} \sqrt{\sigma_{t+1}}\right) \\
& -v_{0}-v_{x} x_{t}-v_{\phi, 1} \phi_{t}-v_{\phi, 2} \phi_{t}^{2}-v_{\sigma, 1} \sqrt{\sigma_{t}}-v_{\sigma, 2} \sigma_{t}^{2}-v_{\phi, \sigma} \phi_{t} \sqrt{\sigma_{t}}+\lambda_{1} \mu_{c} \\
& +\lambda\left(x_{t-1}+\sqrt{\sigma_{t-1}} \varepsilon_{t}^{c}\right)+\sqrt{\sigma_{d}} \sqrt{\sigma_{t-1}} \varepsilon_{t}^{d} \\
= & {\left[K_{0}+K_{1} v_{0}+K_{1} v_{\sigma, 1} \sqrt{\bar{\sigma}}\left(1-\rho_{\sigma}\right)+K_{1} v_{\sigma, 2} \bar{\sigma}\left(1-\rho_{\sigma}\right)-v_{0}+\lambda_{1} \mu_{c}\right]+\left(K_{1} v_{x} \rho_{x}-v_{x}+\lambda\right) x_{t} } \\
& +\left(K_{1} v_{\phi, 1} \rho_{\phi}+K_{1} v_{\phi, \sigma} \sqrt{\bar{\sigma}}\left(1-\rho_{\sigma}\right) \rho_{\phi}-v_{\phi, 1}\right) \phi_{t}+\left(K_{1} v_{\phi, 2} \rho_{\phi}^{2}-v_{\phi, 2}\right) \phi_{t}^{2}
\end{aligned}
$$




$$
\begin{aligned}
& +\left(K_{1} v_{\sigma, 1} \rho_{\sigma}-v_{\sigma, 1}\right) \sqrt{\sigma_{t}}+\left(K_{1} v_{\sigma, 2} \rho_{\sigma}-v_{\sigma, 2}\right) \sigma_{t}+\left(K_{1} v_{\phi, \sigma} \rho_{\sigma} \rho_{\phi}-v_{\phi, \sigma}\right) \phi_{t} \sqrt{\sigma_{t}} \\
& +K_{1}\left[v_{\phi, 1} \sqrt{\sigma_{\phi}}+2 v_{\phi, 2} \rho_{\phi} \sqrt{\sigma_{\phi}} \phi_{t}+v_{\phi, \sigma} \sqrt{\bar{\sigma}}\left(1-\rho_{\sigma}\right) \sqrt{\sigma_{\phi}}+v_{\phi, \sigma} \rho_{\sigma} \sqrt{\sigma_{\phi}} \sqrt{\sigma_{t}}\right] \varepsilon_{t+1}^{\phi} \\
& +K_{1} v_{x} \varphi_{e} \sqrt{\sigma_{t}} \varepsilon_{t+1}^{x}+K_{1} v_{\phi, 2} \sigma_{\phi}\left(\varepsilon_{t+1}^{\phi}\right)^{2}+K_{1}\left(\frac{v_{\sigma, 1}}{2 \sqrt{\bar{\sigma}}}+v_{\sigma, 2}+\frac{v_{\phi, \sigma}}{2 \sqrt{\bar{\sigma}}} \rho_{\phi} \phi_{t}\right) \sqrt{\sigma_{\varepsilon}} \varepsilon_{t+1}^{\sigma} \\
& +K_{1} v_{\phi, \sigma} \frac{\sqrt{\sigma_{\varepsilon} \sigma_{\phi}}}{2 \sqrt{\bar{\sigma}}} \varepsilon_{t+1}^{\sigma} \varepsilon_{t+1}^{\phi}+\lambda \sqrt{\sigma_{t}} \varepsilon_{t+1}^{c}+\sqrt{\sigma_{d}} \sqrt{\sigma_{t}} \varepsilon_{t+1}^{d}
\end{aligned}
$$

\section{A.11.4 Risk-free rate}

The risk-free rate is obtained as

$$
r_{f}=-\log E_{t} \exp \left\{m_{t+1}\right\}=-\log \delta+\mu_{c}+\left(\frac{1}{\theta}-\frac{1}{2}\right) \sigma_{t}+x_{t} .
$$

\section{A.11.5 Conditional equity risk premium}

The conditional expectation of log excess return is as follows:

$$
\begin{aligned}
& E_{t} \log R_{t+1}-r_{f, t}=K_{0}+K_{1} v_{0}+K_{1} v_{\sigma, 1} \sqrt{\bar{\sigma}}\left(1-\rho_{\sigma}\right)+K_{1} v_{\sigma, 2} \bar{\sigma}\left(1-\rho_{\sigma}\right)-v_{0}+\left(\lambda_{1}-1\right) \mu_{c} \\
& +\log \delta+K_{1} v_{\phi, 2} \sigma_{\phi}+\left[K_{1} v_{\phi, 1} \rho_{\phi}+K_{1} v_{\phi, \sigma} \sqrt{\bar{\sigma}}\left(1-\rho_{\sigma}\right) \rho_{\phi}-v_{\phi, 1}\right] \phi_{t}+\left(K_{1} v_{\phi, 2} \rho_{\phi}^{2}-v_{\phi, 2}\right) \phi_{t}^{2} \\
& +\left(K_{1} v_{\sigma, 1} \rho_{\sigma}-v_{\sigma, 1}\right) \sqrt{\sigma_{t}}+\left(K_{1} v_{\sigma, 2} \rho_{\sigma}-v_{\sigma, 2}-\frac{1}{\theta}+\frac{1}{2}\right) \sigma_{t} \\
& +\left(K_{1} v_{\phi, \sigma} \rho_{\sigma} \rho_{\phi}-v_{\phi, \sigma}+\sqrt{\frac{2}{\pi}} K_{1} v_{x} \rho_{\phi} \varphi_{e}\right) \phi_{t} \sqrt{\sigma_{t}}
\end{aligned}
$$

We can study the sign of the coefficient on $\sqrt{\sigma_{t}} \phi_{t}$ :

$$
\begin{aligned}
& K_{1} v_{\phi, \sigma} \rho_{\sigma} \rho_{\phi}-v_{\phi, \sigma}+\sqrt{\frac{2}{\pi}} K_{1} v_{x} \rho_{\phi} \varphi_{e} \\
= & m_{\phi, \sigma}-\frac{B_{\phi} B_{\sigma}}{1-2 C}-0.8 \rho_{\phi}\left(\frac{B \varphi_{e}}{\theta}+K_{1} v_{x} \varphi_{e}\right)+\sqrt{\frac{2}{\pi}} K_{1} v_{x} \rho_{\phi} \varphi_{e} \\
= & \frac{\sigma_{\phi}}{2 \theta^{2}\left(1-2 c_{m}\right)}\left(4 V_{\phi, 2} \rho_{\phi}+\frac{V_{\phi, \sigma}^{2} \rho_{\phi} \sigma_{\varepsilon}}{2 \bar{\sigma} \theta}\right) \cdot\left(0.8 \varphi_{e} B+V_{\phi, \sigma} \rho_{\sigma}\right)-\frac{B_{\phi} B_{\sigma}}{1-2 C}-\left(0.8-\sqrt{\frac{2}{\pi}}\right) \rho_{\phi} K_{1} v_{x} \varphi_{e} \\
= & O\left(\sigma_{\varepsilon}\right)-\left(0.8-\sqrt{\frac{2}{\pi}}\right) \rho_{\phi} K_{1} v_{x} \varphi_{e}
\end{aligned}
$$


When the conditional variance of $\sigma_{t}$, namely $\sigma_{\varepsilon}$, is very small, the coefficient on $\sqrt{\sigma_{t}} \phi_{t}$ is negative.

\section{A.12 Additional calculations for GMM estimation}

In this section we document the analytical solution of the autocorrelation (AC) of consumption growth $\Delta c_{t}$ in which we can match to the estimated autocorrelation of consumption growth from semi-annual data as represented in table A.15.

Mean and Auto-correlation of $x_{t}$. The process for $x_{t}$ follows an AR(1) dynamics with skewnormal innovations

$$
\begin{aligned}
x_{t} & =\mu_{x}\left(1-\rho_{x}\right)+\rho_{x} x_{t-1}+\varphi_{e} \sqrt{\widehat{\sigma}_{t-1}} \varepsilon_{t}^{x} \\
& =\mu_{x}\left(1-\rho_{x}\right)+\rho_{x} x_{t-1}+\varphi_{e} \sqrt{\widehat{\sigma}_{t-1}}\left(\varepsilon_{t}^{x}-\sqrt{\frac{2}{\pi}} \widehat{\phi}_{t-1}\right)+\varphi_{e} \sqrt{\widehat{\sigma}_{t-1}} \sqrt{\frac{2}{\pi}} \widehat{\phi}_{t-1}
\end{aligned}
$$

and $E_{t-1}\left(\varepsilon_{t}^{x}-\sqrt{\frac{2}{\pi}} \widehat{\phi}_{t-1}\right)=0$. Then

$$
\begin{aligned}
E x_{t} & =\mu_{x}\left(1-\rho_{x}\right)+\rho_{x} E x_{t-1}+\varphi_{e} E\left(\sqrt{\widehat{\sigma}_{t-1}} \varepsilon_{t}^{x}\right) \\
& =\mu_{x}\left(1-\rho_{x}\right)+\rho_{x} E x_{t-1}+\varphi_{e} E\left(\sqrt{\widehat{\sigma}_{t-1}} E_{t-1} \varepsilon_{t}^{x}\right) \\
& =\mu_{x}\left(1-\rho_{x}\right)+\rho_{x} E x_{t-1}+\varphi_{e} E\left(\sqrt{\widehat{\sigma}_{t-1}} \sqrt{\frac{2}{\pi}} \widehat{\phi}_{t-1}\right)
\end{aligned}
$$

A first order Taylor expansion of the volatility $\sqrt{\sigma_{t}}$ about $\sqrt{\bar{\sigma}}$ yields

$$
\sqrt{\sigma_{t}} \approx \sqrt{\bar{\sigma}}\left(1-\rho_{\sigma}\right)+\rho_{\sigma} \sqrt{\sigma_{t-1}}+\frac{\sqrt{\sigma_{\varepsilon}}}{2 \sqrt{\bar{\sigma}}} \varepsilon_{t}^{\sigma}
$$

Then

$$
E x_{t}=\mu_{x}\left(1-\rho_{x}\right)+\rho_{x} E x_{t-1}+\varphi_{e} \sqrt{\bar{\sigma}} \sqrt{\frac{2}{\pi}} \bar{\phi}=\mu_{x}+\frac{\varphi_{e} \sqrt{\bar{\sigma}} \sqrt{2 / \pi} \bar{\phi}}{1-\rho_{x}} .
$$

In addition,

$$
\begin{aligned}
& E\left(x_{t}-\mu_{x}-\varphi_{e} \sqrt{\widehat{\sigma}_{t-1}} \sqrt{\frac{2}{\pi}} \widehat{\phi}_{t-1}\right) \cdot\left(x_{t-1}-\mu_{x}\right) \\
= & E\left[\rho_{x}\left(x_{t-1}-\mu_{x}\right)+\varphi_{e} \sqrt{\widehat{\sigma}_{t-1}}\left(\varepsilon_{t}^{x}-\sqrt{\frac{2}{\pi}} \widehat{\phi}_{t-1}\right)\right] \cdot\left(x_{t-1}-\mu_{x}\right)
\end{aligned}
$$




$$
\begin{aligned}
& =\rho_{x} E\left(x_{t-1}-\mu_{x}\right)^{2}+\varphi_{e} E\left[\sqrt{\widehat{\sigma}_{t-1}}\left(x_{t-1}-\mu_{x}\right) E_{t-1}\left(\varepsilon_{t}^{x}-\sqrt{\frac{2}{\pi}} \widehat{\phi}_{t-1}\right)\right] \\
& =\rho_{x} E\left(x_{t-1}-\mu_{x}\right)^{2}
\end{aligned}
$$

Autocorrelation of consumption growth. The dynamics of consumption growth is

$$
\Delta c_{t}=x_{t-1}+\sqrt{\sigma_{t-1}} \varepsilon_{t}^{c}
$$

Then $E \Delta c_{t}=E x_{t-1}=\mu_{x}+\frac{\varphi_{e} \sqrt{\bar{\sigma}} \sqrt{2 / \pi} \bar{\phi}}{1-\rho_{x}}$. We define $\varepsilon_{t}^{x}-\sqrt{\frac{2}{\pi}} \widehat{\phi}_{t-1} \triangleq \xi_{t}^{x}$. Then $E_{t-1} \xi_{t}^{x}=0$ and the dynamics of $x_{t}$ can be rewritten as

$$
x_{t}=\mu_{x}\left(1-\rho_{x}\right)+\rho_{x} x_{t-1}+\varphi_{e} \sqrt{\sigma_{t-1}} \xi_{t}^{x}+\varphi_{e} \sqrt{\sigma_{t-1}} \sqrt{\frac{2}{\pi}} \widehat{\phi}_{t-1}
$$

The autocovariance of $\Delta c_{t}$ is:

$$
\begin{aligned}
& E\left[\left(\Delta c_{t}-E \Delta c_{t}\right)\left(\Delta c_{t-1}-E \Delta c_{t-1}\right)\right] \\
& =E\left[\left(x_{t-1}+\sqrt{\sigma_{t-1}} \varepsilon_{t}^{c}-\mu_{x}-\frac{\varphi_{e} \sqrt{\bar{\sigma}} \sqrt{2 / \pi} \bar{\phi}}{1-\rho_{x}}\right)\left(x_{t-2}+\sqrt{\sigma_{t-2}} \varepsilon_{t-1}^{c}-\mu_{x}-\frac{\varphi_{e} \sqrt{\bar{\sigma}} \sqrt{2 / \pi} \bar{\phi}}{1-\rho_{x}}\right)\right] \\
& =E\left[\left(\rho_{x}\left(x_{t-2}-\mu_{x}\right)+\varphi_{e} \sqrt{\sigma_{t-2}} \xi_{t-1}^{x}+\varphi_{e} \sqrt{\sigma_{t-2}} \sqrt{\frac{2}{\pi}} \widehat{\phi}_{t-2}+\sqrt{\sigma_{t-1}} \varepsilon_{t}^{c}-\frac{\varphi_{e} \sqrt{\bar{\sigma}} \sqrt{2 / \pi} \bar{\phi}}{1-\rho_{x}}\right)\right. \text {. } \\
& \left.\left(x_{t-2}+\sqrt{\sigma_{t-2}} \varepsilon_{t-1}^{c}-\mu_{x}-\frac{\varphi_{e} \sqrt{\bar{\sigma}} \sqrt{2 / \pi} \bar{\phi}}{1-\rho_{x}}\right)\right] \\
& =-2 \rho_{x} \frac{\varphi_{e}^{2} 2 / \pi}{\left(1-\rho_{x}\right)^{2}} \bar{\phi}^{2} \bar{\sigma}+\rho_{x} E\left(x_{t-2}-\mu_{x}\right)^{2}+\rho_{x} E\left[\left(x_{t-2}-\mu_{x}\right) \sqrt{\sigma_{t-2}} E_{t-2} \varepsilon_{t-1}^{c}\right] \\
& +\varphi_{e} E\left[\left(x_{t-2}-\mu_{x}\right) \sqrt{\sigma_{t-2}} E_{t-2} \xi_{t-1}^{x}\right]+\varphi_{e} E\left[\sigma_{t-2} E_{t-2} \varepsilon_{t-1}^{c} \xi_{t-1}^{x}\right]+\varphi_{e} \sqrt{\frac{2}{\pi}} E\left[\left(x_{t-2}-\mu_{x}\right) \sqrt{\sigma_{t-2}} \widehat{\phi}_{t-2}\right] \\
& +\varphi_{e} \sqrt{\frac{2}{\pi}} E\left[\sigma_{t-2} \widehat{\phi}_{t-2} E_{t-2} \varepsilon_{t-1}^{c}\right]+E\left[\left(x_{t-2}-\mu_{x}\right) \sqrt{\sigma_{t-1}} E_{t-1} \varepsilon_{t}^{c}+\sqrt{\sigma_{t-2}} \sqrt{\sigma_{t-1}} \varepsilon_{t-1}^{c} E_{t-1} \varepsilon_{t}^{c}\right] \\
& =-2 \rho_{x} \frac{\varphi_{e}^{2}(2 / \pi)}{\left(1-\rho_{x}\right)^{2}} \bar{\phi}^{2} \bar{\sigma}+\rho_{x} E\left(x_{t-2}-\mu_{x}\right)^{2}+\varphi_{e} \sqrt{\frac{2}{\pi}} E\left[\left(x_{t-2}-\mu_{x}\right) \sqrt{\sigma_{t-2}} \widehat{\phi}_{t-2}\right]
\end{aligned}
$$

Let's focus on the last term $E_{x \sigma \phi} \triangleq E\left[\left(x_{t}-\mu_{x}\right) \sqrt{\sigma_{t}} \widehat{\phi}_{t}\right]$. First, we rewrite $x_{t}-\mu_{x}$ as:

$$
\begin{aligned}
x_{t}-\mu_{x} & =\rho_{x}\left(x_{t-1}-\mu_{x}\right)+\varphi_{e} \sqrt{\sigma_{t-1}} \xi_{t}^{x}+\varphi_{e} \sqrt{\frac{2}{\pi}} \sqrt{\sigma_{t-1}} \widehat{\phi}_{t-1} \\
& =\varphi_{e} \sum_{i=0}^{\infty} \rho_{x}^{i} \sqrt{\sigma_{t-1-i}} \xi_{t-i}^{x}+\varphi_{e} \sqrt{\frac{2}{\pi}} \sum_{i=0}^{\infty} \rho_{x}^{i} \sqrt{\sigma_{t-1-i}} \widehat{\phi}_{t-1-i}
\end{aligned}
$$


which implies that

$$
E_{x \sigma \phi}=\varphi_{e} \sqrt{\frac{2}{\pi}} \sum_{i=0}^{\infty} \rho_{x}^{i} E\left[\left(\sqrt{\sigma_{t-1-i}} \widehat{\phi}_{t-1-i}\right)\left(\sqrt{\sigma_{t}} \widehat{\phi}_{t}\right)\right]
$$

That is because $E\left(\varphi_{e} \rho_{x}^{i} \sqrt{\sigma_{t-1-i}} \xi_{t-i}^{x}\right)\left(\sqrt{\sigma_{t}} \widehat{\phi}_{t}\right)=0, \forall i$. In order to fully characterize equation (A.21), we need to calculate

$$
\begin{aligned}
& E\left[\left(\sqrt{\sigma_{t-i}} \widehat{\phi}_{t-i}\right)\left(\sqrt{\sigma_{t}} \widehat{\phi}_{t}\right)\right] \\
= & E\left[\left(\sqrt{\sigma_{t-i}} \widehat{\phi}_{t-i}\right) E_{t-i}\left(\sqrt{\sigma_{t}}\left(\bar{\phi}\left(1-\rho_{\phi}\right) \sum_{j=0}^{i-1} \rho_{\phi}^{j}+\rho_{\phi}^{i} \widehat{\phi}_{t-i}+\rho_{\phi} \sqrt{\sigma_{\phi}} \sum_{j=0}^{i-1} \varepsilon_{t-j}^{\phi} \rho_{\phi}^{j}\right)\right]\right] \\
= & E\left[\left(\sqrt{\sigma_{t-i}} \widehat{\phi}_{t-i}\right)\left(\bar{\phi}\left(1-\rho_{\phi}\right) \sum_{j=0}^{i-1} \rho_{\phi}^{j}+\rho_{\phi}^{i} \widehat{\phi}_{t-i}\right) E_{t-i} \sqrt{\sigma_{t}}\right] \\
= & E\left[\sqrt{\sigma_{t-i}} \widehat{\phi}_{t-i}\left(\bar{\phi}\left(1-\rho_{\phi}\right) \sum_{j=0}^{i-1} \rho_{\phi}^{j}+\rho_{\phi}^{i} \widehat{\phi}_{t-i}\right) \cdot\left(\sqrt{\bar{\sigma}}\left(1-\rho_{\sigma}\right) \sum_{j=0}^{i-1} \rho_{\sigma}^{j}+\rho_{\sigma}^{i} \sqrt{\sigma_{t-i}}\right)\right] \\
= & E\left[\bar{\phi}\left(1-\rho_{\phi}\right)\left(\sum_{j=0}^{i-1} \rho_{\phi}^{j}\right) \sqrt{\bar{\sigma}}\left(1-\rho_{\sigma}\right)\left(\sum_{j=0}^{i-1} \rho_{\sigma}^{j}\right) \sqrt{\sigma_{t-i}} \widehat{\phi}_{t-i}\right]+\left(\rho_{\phi} \rho_{\sigma}\right)^{i} E\left[\sigma_{t-i} \widehat{\phi}_{t-i}^{2}\right] \\
+ & \rho_{\sigma}^{i} \bar{\phi}\left(1-\rho_{\phi}\right)\left(\sum_{j=0}^{i-1} \rho_{\phi}^{j}\right) E\left[\sigma_{t-i} \widehat{\phi}_{t-i}\right]+\rho_{\phi}^{i} \sqrt{\bar{\sigma}}\left(1-\rho_{\sigma}\right)\left(\sum_{j=0}^{i-1} \rho_{\sigma}^{j}\right) E\left[\sqrt{\sigma_{t-i}} \widehat{\phi}_{t-i}^{2}\right]
\end{aligned}
$$

Given the independence between skewness and variance variables, the above equation becomes:

$$
\begin{aligned}
& E\left[\left(\sqrt{\sigma_{t-i}} \widehat{\phi}_{t-i}\right)\left(\sqrt{\sigma_{t}} \widehat{\phi}_{t}\right)\right] \\
= & \bar{\phi}^{2} \bar{\sigma}\left(1-\rho_{\phi}\right)\left(1-\rho_{\sigma}\right)\left(\sum_{j=0}^{i-1} \rho_{\phi}^{j}\right)\left(\sum_{j=0}^{i-1} \rho_{\sigma}^{j}\right)+\rho_{\sigma}^{i} \bar{\phi}^{2} \bar{\sigma}\left(1-\rho_{\phi}\right)\left(\sum_{j=0}^{i-1} \rho_{\phi}^{j}\right) \\
& +\rho_{\phi}^{i} \bar{\sigma}\left(1-\rho_{\sigma}\right)\left(\sum_{j=0}^{i-1} \rho_{\sigma}^{j}\right)\left(\frac{\rho_{\phi}^{2} \sigma_{\phi}}{1-\rho_{\phi}^{2}}+\bar{\phi}^{2}\right)+\left(\rho_{\phi} \rho_{\sigma}\right)^{i} \bar{\sigma}\left(\frac{\rho_{\phi}^{2} \sigma_{\phi}}{1-\rho_{\phi}^{2}}+\bar{\phi}^{2}\right) \\
= & \bar{\phi}^{2} \bar{\sigma}\left(1-\rho_{\phi}^{i}\right)\left(1-\rho_{\sigma}^{i}\right)+\rho_{\sigma}^{i} \bar{\phi}^{2} \bar{\sigma}\left(1-\rho_{\phi}^{i}\right)+\rho_{\phi}^{i} \bar{\sigma}\left(1-\rho_{\sigma}^{i}\right)\left(\frac{\rho_{\phi}^{2} \sigma_{\phi}}{1-\rho_{\phi}^{2}}+\bar{\phi}^{2}\right)+\left(\rho_{\phi} \rho_{\sigma}\right)^{i} \bar{\sigma}\left(\frac{\rho_{\phi}^{2} \sigma_{\phi}}{1-\rho_{\phi}^{2}}+\bar{\phi}^{2}\right) \\
= & \bar{\phi}^{2} \bar{\sigma}+\rho_{\phi}^{i} \bar{\sigma} \frac{\rho_{\phi}^{2} \sigma_{\phi}}{1-\rho_{\phi}^{2}}
\end{aligned}
$$


This implies that equation (A.21) can be written as:

$$
\begin{aligned}
E_{x \sigma \phi} & =\varphi_{e} \sqrt{\frac{2}{\pi}} \sum_{i=0}^{\infty} \rho_{x}^{i} E\left[\left(\sqrt{\sigma_{t-1-i}} \widehat{\phi}_{t-1-i}\right)\left(\sqrt{\sigma_{t}} \widehat{\phi}_{t}\right)\right]=\varphi_{e} \sqrt{\frac{2}{\pi}} \sum_{i=1}^{\infty} \rho_{x}^{i-1}\left(\bar{\phi}^{2} \bar{\sigma}+\rho_{\phi}^{i} \bar{\sigma} \frac{\rho_{\phi}^{2} \sigma_{\phi}}{1-\rho_{\phi}^{2}}\right) \\
& =\varphi_{e} \sqrt{\frac{2}{\pi}}\left[\frac{\bar{\phi}^{2} \bar{\sigma}}{1-\rho_{x}}+\frac{\bar{\sigma} \rho_{\phi}^{3} \sigma_{\phi}}{\left(1-\rho_{\phi}^{2}\right)\left(1-\rho_{x} \rho_{\phi}\right)}\right]
\end{aligned}
$$

Going back to the expression of autocovariance of $\Delta c_{t}$ given in equation (A.20), the last moment that we need to compute is

$$
\begin{aligned}
E_{x x} & \triangleq E\left(x_{t}-\mu_{x}\right)^{2}=E\left[\rho_{x}\left(x_{t-1}-\mu_{x}\right)+\varphi_{e} \sqrt{\sigma_{t-1}} \xi_{t}^{x}+\varphi_{e} \sqrt{\sigma_{t-1}} \sqrt{\frac{2}{\pi}} \widehat{\phi}_{t-1}\right]^{2} \\
& =\rho_{x}^{2} E\left(x_{t-1}-\mu_{x}\right)^{2}+\varphi_{e}^{2} E\left[\sigma_{t-1} E_{t-1}\left(\xi_{t}^{x}\right)^{2}\right]+\varphi_{e}^{2} \frac{2}{\pi} E\left(\sigma_{t-1} \widehat{\phi}_{t-1}^{2}\right)+2 \varphi_{e} \sqrt{\frac{2}{\pi}} \rho_{x} E\left(x_{t-1}-\mu_{x}\right) \\
& =\frac{1}{1-\rho_{x}^{2}}\left[\varphi_{e}^{2} E\left[\sigma_{t-1}\left(1-\frac{2}{\pi} \widehat{\phi}_{t-1}^{2}\right)\right]+\varphi_{e}^{2} \frac{2}{\pi} \bar{\sigma}\left(\bar{\phi}^{2}+\frac{\rho_{\phi}^{2} \sigma_{\phi}}{1-\rho_{\phi}^{2}}\right)+2 \varphi_{e} \sqrt{\frac{2}{\pi}} \rho_{x} E_{x \sigma \phi}\right] \\
& =\frac{1}{1-\rho_{x}^{2}}\left(\varphi_{e}^{2} \bar{\sigma}+\frac{2}{\pi} \varphi_{e}^{2} \bar{\sigma} \frac{\rho_{\phi}^{2} \sigma_{\phi}}{1-\rho_{\phi}^{2}}+2 \varphi_{e} \sqrt{\frac{2}{\pi}} \rho_{x} E_{x \sigma \phi}\right)
\end{aligned}
$$

The variance of consumption growth $\Delta c_{t}$ is:

$$
\begin{aligned}
E\left(\Delta c_{t}-E \Delta c_{t}\right)^{2} & =E\left[x_{t-1}-\mu_{x}+\sqrt{\sigma_{t-1}} \varepsilon_{t}^{c}-\frac{\varphi_{e} \sqrt{2 / \pi} \bar{\phi} \sqrt{\bar{\sigma}}}{1-\rho_{x}}\right]^{2} \\
& =E\left(x_{t-1}-\mu_{x}\right)^{2}+\bar{\sigma}+\frac{\varphi_{e}^{2}(2 / \pi) \bar{\phi}^{2} \bar{\sigma}}{\left(1-\rho_{x}\right)^{2}}-2 \frac{\varphi_{e}^{2}(2 / \pi) \bar{\phi}^{2} \bar{\sigma}}{\left(1-\rho_{x}\right)^{2}} \\
& =E_{x x}+\bar{\sigma}-\frac{\varphi_{e}^{2}(2 / \pi) \bar{\phi}^{2} \bar{\sigma}}{\left(1-\rho_{x}\right)^{2}}
\end{aligned}
$$

Hence, the first order $\mathrm{AC}$ of $\Delta c_{t}$ is

$$
\begin{aligned}
A C_{1}\left(\Delta c_{t}\right) & =\frac{E\left[\left(\Delta c_{t}-E \Delta c_{t}\right)\left(\Delta c_{t-1}-E \Delta c_{t-1}\right)\right]}{V\left(\Delta c_{t}\right)} \\
& =\frac{-2 \rho_{x} \frac{\varphi_{e}^{2}(2 / \pi)}{\left(1-\rho_{x}\right)^{2}} \bar{\phi}^{2} \bar{\sigma}+\rho_{x} E_{x x}+\varphi_{e} \sqrt{\frac{2}{\pi}} E_{x \sigma \phi}}{E_{x x}+\bar{\sigma}-\frac{\varphi_{e}^{2}(2 / \pi) \bar{\phi}^{2} \bar{\sigma}}{\left(1-\rho_{x}\right)^{2}}}
\end{aligned}
$$

In our benchmark model, we set $\bar{\phi}=0$, then the expression of $E_{x \sigma \phi}, E_{x x}$ in equation (A.21) 
Table A.15: Summary Statistics of Consumption Growth Rate

\begin{tabular}{ccccc}
\hline$\mu(\Delta c)$ & $\sigma(\Delta c)$ & Skew $(\Delta c)$ & Kurt $(\Delta c)$ & $A C_{1}(\Delta c)$ \\
\hline 0.009 & $6.39 \times 10^{-5}$ & -0.447 & 3.984 & 0.477 \\
$(0.001)$ & $\left(1.04 \times 10^{-5}\right)$ & $(0.263)$ & $(0.453)$ & $(0.084)$ \\
\hline
\end{tabular}

Notes -The table reports the unconditional mean, standard deviation, skewness, kurtosis, and first order autocorrelation for the real log-consumption growth rate using US semi-annual data from 1951S2 to 2011S1. Consumption is nondurables and services consumption.

and (A.22) can be simplified as:

$$
\begin{aligned}
E_{x \sigma \phi} & =\varphi_{e} \sqrt{\frac{2}{\pi}} \frac{\bar{\sigma} \rho_{\phi}^{3} \sigma_{\phi}}{\left(1-\rho_{\phi}^{2}\right)\left(1-\rho_{x} \rho_{\phi}\right)} \\
E_{x x} & =\frac{1}{1-\rho_{x}^{2}}\left(\varphi_{e}^{2} \bar{\sigma}+\frac{2}{\pi} \varphi_{e}^{2} \bar{\sigma} \frac{\rho_{\phi}^{2} \sigma_{\phi}}{1-\rho_{\phi}^{2}}+2 \varphi_{e} \sqrt{\frac{2}{\pi}} \rho_{x} E_{x \sigma \phi}\right) \\
& =\frac{\varphi_{e}^{2} \bar{\sigma}}{1-\rho_{x}^{2}}\left[1+\frac{2}{\pi} \frac{\rho_{\phi}^{2} \sigma_{\phi}}{\left(1-\rho_{\phi}^{2}\right)\left(1-\rho_{x} \rho_{\phi}\right)} \cdot\left(1+\rho_{x} \rho_{\phi}\right)\right]
\end{aligned}
$$

And hence, equation (A.23) is:

$$
A C_{1}\left(\Delta c_{t}\right)=\frac{\varphi_{e}^{2}\left[\rho_{x}+\frac{2}{\pi} \frac{\rho_{\phi}^{2} \sigma_{\phi}}{\left(1-\rho_{\phi}^{2}\right)\left(1-\rho_{x} \rho_{\phi}\right)} \cdot\left(\rho_{x}+\rho_{\phi}\right)\right]}{1-\rho_{x}^{2}+\varphi_{e}^{2}\left[1+\frac{2}{\pi} \frac{\rho_{\phi}^{2} \sigma_{\phi}}{\left(1-\rho_{\phi}^{2}\right)\left(1-\rho_{x} \rho_{\phi}\right)} \cdot\left(1+\rho_{x} \rho_{\phi}\right)\right]} \triangleq \frac{\varphi_{e}^{2} K_{N}}{1-\rho_{x}^{2}+\varphi_{e}^{2} K_{D}}
$$

Given $A C_{1}\left(\Delta c_{t}\right)$ estimated from data and GMM estimates of all other parameters, we can solve for $\varphi_{e}$ :

$$
\varphi_{e}=\sqrt{\frac{A C_{1}\left(1-\rho_{x}^{2}\right)}{K_{N}-A C_{1} K_{D}}}
$$

where

$K_{N}=\rho_{x}+\frac{2}{\pi} \frac{\rho_{\phi}^{2} \sigma_{\phi}}{\left(1-\rho_{\phi}^{2}\right)\left(1-\rho_{x} \rho_{\phi}\right)} \cdot\left(\rho_{x}+\rho_{\phi}\right), \quad K_{D}=1+\frac{2}{\pi} \frac{\rho_{\phi}^{2} \sigma_{\phi}}{\left(1-\rho_{\phi}^{2}\right)\left(1-\rho_{x} \rho_{\phi}\right)} \cdot\left(1+\rho_{x} \rho_{\phi}\right)$ 


\section{A.13 Numerical algorithm}

Utility function. We shall solve for the remainder of the utility function $\widetilde{V}_{t}$ in the Bellman equation (A.2) via value function iteration. In order to compute the conditional expectation on the right hand side of (A.2) we will make use of the properties of the skew-normal distribution that we have reported in Lemma 1. Specifically, if $z$ follows skew normal distribution $S K N(0,1, \nu)$, then

$$
\log E_{t} \exp \left\{\kappa_{1} z\right\}=\log (2)+\frac{\kappa_{1}^{2}}{2}+\log \Phi\left(\frac{\nu \kappa_{1}}{\sqrt{1+\nu^{2}}}\right)
$$

We rewrite the right hand side of the Bellman equation (A.2) as:

$$
\begin{aligned}
\widetilde{V}\left(\sigma_{t}^{c}, \nu_{t}\right)= & \delta \theta \log E_{t}\left\{E_{t}\left[\exp \left\{\frac{\mu_{c}+\varphi_{e} B \sqrt{\sigma_{t}^{x}} \varepsilon_{t+1}^{x}+\sqrt{\sigma_{t}^{c}} \varepsilon_{t+1}^{c}+\widetilde{V}_{t+1}}{\theta}\right\} \mid \nu_{t+1}\right]\right\} \\
= & \delta \mu_{c}+\frac{\delta}{2 \theta} \sigma_{t}^{c}+\delta \theta\left(\log (2)+\frac{\varphi_{e}^{2} B^{2} \sigma_{t}^{x}}{2 \theta^{2}}\right)+\delta \theta \log E_{t} \Phi\left(\frac{\nu_{t+1} \varphi_{e} B \sqrt{\sigma_{t}^{x}}}{\theta \sqrt{1+\nu_{t+1}^{2}}}\right) \\
& +\delta \theta \log E_{t} \exp \left\{\frac{\widetilde{V}\left(\sigma_{t+1}^{c}, \nu_{t+1}\right)}{\theta}\right\} .
\end{aligned}
$$

Note that $\sigma_{t}^{x}$ that appears on the right hand side of the previous equation is a function of $\sigma_{t}^{c}$ and $\nu_{t}$ only:

$$
\sigma_{t}^{x}=\sigma_{t}^{c} /\left[1-\frac{2}{\pi}\left(E_{t} \phi_{t+1}\right)^{2}\right]
$$

where $\phi_{t}=\frac{\nu_{t}}{\sqrt{1+\nu_{t}^{2}}}$ is a function of $\nu_{t}$. And hence, $\widetilde{V}_{t}$ is a function of $\sigma_{t}^{c}$ and $\phi_{t}$ only.

We assume that both $\sigma_{t}^{c}$ and $\phi_{t}$ follow $A R(1)$ processes, that is

$$
\begin{aligned}
\sigma_{t}^{c} & =\left(1-\rho_{\sigma}\right) \bar{\sigma}_{c}+\rho_{\sigma} \sigma_{t-1}^{c}+\sqrt{\sigma_{\varepsilon}} \varepsilon_{t}^{\sigma}, \\
\phi_{t} & =\rho_{\phi} \phi_{t-1}+\sqrt{\sigma_{\phi}} \varepsilon_{t}^{\phi} .
\end{aligned}
$$

where the innovations $\varepsilon_{t}^{\sigma}$ and $\varepsilon_{t}^{\phi}$ are $i . i . d$ distributed as standard normal. We approximate $\sigma_{t}^{c}$ and $\phi_{t}$ on discrete grids and assume independent Markov transition processes for their dynamics. Specifically, we adopt the Rouwenhorst method (see Kopecky and Suen (2010) for details) to approximate $\operatorname{AR}(1)$ transition dynamics with various degrees of persistence. We approximate the $\sigma_{t}^{c}$ process by a 
symmetric and evenly-spaced state space $Y_{N}=\left\{y_{1}, \ldots, y_{N}\right\}$, with $N=21$ defined over the interval $[0,2 \psi]$. The transition matrix $\Theta_{N}$ with two parameters $p, q \in(0,1)$ is defined recursively as follows:

$$
p\left[\begin{array}{cc}
\Theta_{N-1} & \mathbf{0} \\
\mathbf{0}^{\prime} & 0
\end{array}\right]+(1-p)\left[\begin{array}{cc}
\mathbf{0} & \Theta_{N-1} \\
0 & \mathbf{0}^{\prime}
\end{array}\right]+q\left[\begin{array}{cc}
\mathbf{0}^{\prime} & 0 \\
\Theta_{N-1} & \mathbf{0}
\end{array}\right]+(1-q)\left[\begin{array}{cc}
0 & \mathbf{0}^{\prime} \\
\mathbf{0} & \Theta_{N-1}
\end{array}\right]
$$

where

$$
\Theta_{2}=\left[\begin{array}{cc}
p & 1-p \\
1-q & q
\end{array}\right]
$$

and 0 is an $(N-1)$-by-1 vector of zeros. Kopecky and Suen (2010) show that using the persistence $\rho_{\sigma}$ and shock volatility $\sigma_{\sigma}$ alone, it is possible to construct the approximate Markov chain, with $p=q=\left(1+\rho_{\sigma}\right) / 2$ and $\psi=\sqrt{(N-1) \sigma_{\varepsilon} /\left(1-\rho_{\sigma}^{2}\right)}$. Following the same procedure, we approximate the process of the variable that governs the skewness dynamics, $\phi_{t}$, with a symmetric state space $Z_{N}=$ $\left\{z_{1}, \ldots, z_{N}\right\}$ with $N=5$ evenly spaced nodes over the interval $[-\tilde{\psi}, \tilde{\psi}]$ with $p=q=\left(1+\rho_{\phi}\right) / 2$ and $\tilde{\psi}=\sqrt{(N-1) \sigma_{\phi} /\left(1-\rho_{\phi}^{2}\right)}$. Using the approximation of $\operatorname{AR}(1)$ transition dynamics, we can solve the Bellman equation (A.2) via value function iteration.

Price-dividend ratio. We consider the returns to a claim to the following dividend process:

$$
\Delta d_{t}=\lambda_{1} \mu_{c}+\lambda\left(x_{t-1}+\sqrt{\sigma_{t-1}^{c}} \varepsilon_{t}^{c}\right)+\sqrt{\sigma_{d}} \sqrt{\sigma_{t-1}^{c}} \varepsilon_{t}^{d}, \text { where } \varepsilon_{t}^{d} \sim N(0,1)
$$

$\lambda>1$ denotes the leverage ratio for the claim on consumption. Then the returns to the levered consumption claim satisfy

$$
\begin{aligned}
1 & =E_{t}\left[\exp \left\{m_{t+1}\right\}\left(\frac{P_{t+1}+D_{t+1}}{P_{t}}\right)\right] \\
& =E_{t}\left[\exp \left\{m_{t+1}\right\}\left(\frac{1+\exp \left\{V_{d, t}\right\}}{\exp \left\{V_{d, t}\right\}}\right) \exp \left\{\lambda_{1} \mu_{c}+\lambda\left(x_{t}+\sqrt{\sigma_{t}^{c}} \varepsilon_{t+1}^{c}\right)+\sqrt{\sigma_{d}} \sqrt{\sigma_{t}^{c}} \varepsilon_{t+1}^{d}\right\}\right]
\end{aligned}
$$

where $v_{d, t}=\log \left(P_{t} / D_{t}\right)$ denotes the $\log$ ratio of price to the dividend. Rewrite the Euler equation as:

$$
\begin{aligned}
v_{d, t}= & \log E_{t} \exp \left\{m_{t+1}+\lambda_{1} \mu_{c}+\lambda\left(x_{t}+\sqrt{\sigma_{t}^{c}} \varepsilon_{t+1}^{c}\right)+\sqrt{\sigma_{d}} \sqrt{\sigma_{t}^{c}} \varepsilon_{t+1}^{d}\right\}\left(1+\exp \left\{v_{d, t+1}\right\}\right) \\
= & \log \left(E_{t} \exp \left\{m_{t+1}+\lambda_{1} \mu_{c}+\lambda\left(x_{t}+\sqrt{\sigma_{t}^{c}} \varepsilon_{t+1}^{c}\right)+\sqrt{\sigma_{d}} \sqrt{\sigma_{t}^{c}} \varepsilon_{t+1}^{d}\right\}\right. \\
& \left.+E_{t} \exp \left\{m_{t+1}+\lambda_{1} \mu_{c}+\lambda\left(x_{t}+\sqrt{\sigma_{t}^{c}} \varepsilon_{t+1}^{c}\right)+\sqrt{\sigma_{d}} \sqrt{\sigma_{t}^{c}} \varepsilon_{t+1}^{d}+V_{d, t+1}\right\}\right)
\end{aligned}
$$


Using the equation for the stochastic discount factor (A.3), the first part in the logarithm of equation (A.25) is:

$$
\begin{aligned}
& E_{t} \exp \left\{m_{t+1}+\lambda_{1} \mu_{c}+\lambda\left(x_{t}+\sqrt{\sigma_{t}^{c}} \varepsilon_{t+1}^{c}\right)+\sqrt{\sigma_{d}} \sqrt{\sigma_{t}^{c}} \varepsilon_{t+1}^{d}\right\} \\
= & E_{t} \exp \left\{\log \delta+\left(\lambda_{1}-1+\frac{1}{\theta}\right) \mu_{c}+\left(\lambda-1+\frac{1}{\theta}\right)\left(x_{t}+\sqrt{\sigma_{t}^{c}} \varepsilon_{t+1}^{c}\right)+\sqrt{\sigma_{d}} \sqrt{\sigma_{t}^{c}} \varepsilon_{t}^{d}\right. \\
& \left.+\frac{1}{\theta}\left(\widetilde{V}_{t+1}-\frac{\widetilde{V}_{t}}{\delta}\right)+\frac{B}{\theta}\left(\rho_{x} x_{t}+\varphi_{e} \sqrt{\sigma_{t}^{x}} \varepsilon_{t+1}^{x}-\frac{x_{t}}{\delta}\right)\right\} \\
= & 2 \delta \exp \left\{\left(\lambda_{1}-1+\frac{1}{\theta}\right) \mu_{c}+(\lambda-1) x_{t}-\frac{\widetilde{V}_{t}}{\delta \theta}+\frac{1}{2}\left(\lambda-1+\frac{1}{\theta}\right)^{2} \sigma_{t}^{c}+\frac{\sigma_{d} \sigma_{t}^{c}}{2}+\frac{B^{2} \varphi_{e}^{2}}{2 \theta^{2}} \sigma_{t}^{x}\right\} . \\
& E_{t}\left[\Phi\left(\frac{B \varphi_{e} \sqrt{\sigma_{t}^{x}} \nu_{t+1}}{\theta \sqrt{1+\nu_{t+1}^{2}}}\right) \cdot \exp \left\{\frac{\widetilde{V}_{t+1}}{\theta}\right\}\right] .
\end{aligned}
$$

Note that the right hand side of $v_{d, t}$ in (A.25) is a function of state variables $\sigma_{t}^{c}, \phi_{t}, x_{t}$, where $\phi_{t}=$ $\nu_{t} / \sqrt{1+\nu_{t}^{2}}$. We approximate $v_{d, t}$ using a quadratic polynomial function of these three state variables $x_{t}, \sqrt{\sigma_{t}^{c}}$, and $\phi_{t}$ :

$$
v_{d, t}=a_{1}+a_{2} x_{t}+a_{3} x_{t}^{2}+a_{4} \sqrt{\sigma_{t}^{c}}+a_{5} \sigma_{t}^{c}+a_{6} \phi_{t}+a_{7} \phi_{t}^{2}+a_{8} x_{t} \sqrt{\sigma_{t}^{c}}+a_{9} x_{t} \phi_{t}+a_{10} \sqrt{\sigma_{t}^{c}} \phi_{t} .
$$

Substituting the guess of $v_{d, t}$ function into the second part in the logarithm of equation (A.25), we have:

$$
\begin{aligned}
& E_{t} \exp \left\{m_{t+1}+\lambda_{1} \mu_{c}+\lambda\left(x_{t}+\sqrt{\sigma_{t}^{c}} \varepsilon_{t+1}^{c}\right)+\sqrt{\sigma_{d}} \sqrt{\sigma_{t}^{c}} \varepsilon_{t+1}^{d}+V_{d, t+1}\right\} \\
= & \delta \exp \left\{\left(\lambda_{1}-1+\frac{1}{\theta}\right) \mu_{c}+(\lambda-1) x_{t}-\frac{\widetilde{V}_{t}}{\delta \theta}+\frac{1}{2}\left(\lambda-1+\frac{1}{\theta}\right)^{2} \sigma_{t}^{c}+\frac{\sigma_{d} \sigma_{t}^{c}}{2}\right\} . \\
& E_{t} \exp \left\{\frac{B \varphi_{e} \sqrt{\sigma_{t}^{x}}}{\theta} \varepsilon_{t+1}^{x}+\frac{\widetilde{V}_{t+1}}{\theta}+V_{d, t+1}\right\} \\
= & \delta \exp \left\{\left(\lambda_{1}-1+\frac{1}{\theta}\right) \mu_{c}+(\lambda-1) x_{t}-\frac{\widetilde{V}_{t}}{\delta \theta}+\frac{1}{2}\left(\lambda-1+\frac{1}{\theta}\right)^{2} \sigma_{t}^{c}+\frac{\sigma_{d} \sigma_{t}^{c}}{2}\right\} \\
& \cdot E_{t} \exp \left\{\frac{\tilde{V}_{t+1}}{\theta}+a_{1}+a_{2} \rho_{x} x_{t}+a_{3} \rho_{x}^{2} x_{t}^{2}+a_{4} \sqrt{\sigma_{t+1}^{c}}+a_{5} \sigma_{t+1}^{c}+a_{6} \phi_{t+1}+a_{7} \phi_{t+1}^{2}\right. \\
& +a_{8} \rho_{x} x_{t} \sqrt{\sigma_{t+1}^{c}}+a_{9} \rho_{x} x_{t} \phi_{t+1}+a_{10} \sqrt{\sigma_{t+1}^{c}} \phi_{t+1}+a_{3} \varphi_{e}^{2} \sigma_{t}^{x}\left(\varepsilon_{t+1}^{x}\right)^{2}
\end{aligned}
$$




$$
\left.+\left(\frac{B \varphi_{e} \sqrt{\sigma_{t}^{x}}}{\theta}+a_{2} \varphi_{e} \sqrt{\sigma_{t}^{x}}+2 a_{3} \rho_{x} \varphi_{e} \sqrt{\sigma_{t}^{x}} x_{t}+a_{8} \varphi_{e} \sqrt{\sigma_{t}^{x}} \sqrt{\sigma_{t+1}^{c}}+a_{9} \varphi_{e} \sqrt{\sigma_{t}^{x}} \phi_{t+1}\right) \varepsilon_{t+1}^{x}\right\}
$$

We solve for parameters $a_{1}, a_{2}, a_{3}, a_{4}, a_{5}, a_{6}, a_{7}, a_{8}, a_{9}, a_{10}$, by regressing $v_{d, t}$ on these three state variables and all their quadratic terms $\left[1, x_{t}, x_{t}^{2}, \sqrt{\sigma_{t}^{c}}, \sigma_{t}^{c}, \phi_{t}, \phi_{t}^{2}, x_{t} \sqrt{\sigma_{t}^{c}}, x_{t} \phi_{t}, \sqrt{\sigma_{t}^{c}} \phi_{t}\right]$, and updating regression coefficients via iteration.

\section{A.14 Comparison of approximations}

\section{A.14.1 Constant volatility case}

In this subsection we examine two alternative analytical solutions for the case with time-varying mean and skewness described in section A.3 of the Technical Appendix. The main purpose of this exercise is to measure the consistency of magnitudes and signs of the key parameters on the value function, the log-price to dividend ratio, and the expected excess return. After showing that the analytical solution we applied in the dissertation is robust with respect to these alternatives, we compare the analytical and numerical solutions of the value function $\widetilde{V}_{t}$ and $v_{d, t}$ with a variety of calibrations for the $\phi_{t}$ process. This exercise demonstrates that the analytical solution is reliable to illustrate the role of key parameters in determining the value function, the log price to dividend ratio, and the expected excess returns.

\section{First alternative analytical solution}

This approximation differs from the one used in section A.3 of the Technical Appendix in that we do not linearize the logarithm in the second line of equation (A.16). The main departure starts in equation (A.28) of what follows.

Utility function. We shall solve for the Bellman equation (A.4)

$$
\widetilde{V}_{t}=\delta \theta \log E_{t} \exp \left\{\frac{\mu_{c}+\varphi_{e} B \sqrt{\bar{\sigma}} \varepsilon_{t+1}^{x}+\sqrt{\bar{\sigma}} \varepsilon_{t+1}^{c}+\widetilde{V}_{t+1}}{\theta}\right\}
$$

using the properties of the properties of the skew-normal distribution documented in Lemma A.1.1, that is, if $z$ follows skew normal distribution $S K N(0,1, \nu)$, then

$$
\log E_{t} \exp \left\{\kappa_{1} z\right\}=\log (2)+\frac{\kappa_{1}^{2}}{2}+\log \Phi\left(\frac{\nu \kappa_{1}}{\sqrt{1+\nu^{2}}}\right)
$$


Using Lew's approximation, we obtain

$$
\log E_{t} \exp \left\{\kappa_{1} z\right\} \quad \approx \frac{1}{2} \kappa_{1}^{2}+\log \left(1+0.8 \kappa_{1} \phi_{t}\right)
$$

Use the law of iterated expectations to compute

$$
\begin{aligned}
\widetilde{V}_{t} & =\delta \theta \log E_{t} E_{t}\left[\exp \left\{\frac{1}{\theta}\left(\mu_{c}+\varphi_{e} B \sqrt{\bar{\sigma}} \varepsilon_{t+1}^{x}+\sqrt{\bar{\sigma}} \varepsilon_{t+1}^{c}+\widetilde{V}_{t+1}\right)\right\} \mid\left(\varepsilon_{t+1}^{c}, \widetilde{V}_{t+1}\right)\right] \\
& =\delta \theta \log E_{t} \exp \left\{\frac{\mu_{c}}{\theta}+\frac{\varphi_{e}^{2} B^{2} \bar{\sigma}}{2 \theta^{2}}+\log \left(1+\frac{0.8 \varphi_{e} B \sqrt{\bar{\sigma}}}{\theta} \phi_{t+1}\right)+\frac{\sqrt{\bar{\sigma}} \varepsilon_{t+1}^{c}}{\theta}+\frac{\widetilde{V}_{t+1}}{\theta}\right\}
\end{aligned}
$$

We guess that the solution of this value function is linear in $\phi_{t}: \widetilde{V}_{t}=V_{0}+V_{\phi} \phi_{t}$. By plugging in our guess in the value function above, we get:

$$
\begin{aligned}
\widetilde{V}_{t} & =\delta \theta \log E_{t}\left[\exp \left\{\frac{\mu_{c}}{\theta}+\frac{\varphi_{e}^{2} B^{2} \bar{\sigma}}{2 \theta^{2}}+\log \left(1+A \rho_{\phi} \phi_{t}+A \sqrt{\sigma_{\phi}} \varepsilon_{t+1}^{\phi}\right)+\frac{\sqrt{\bar{\sigma}} \varepsilon_{t+1}^{c}}{\theta}+\frac{V_{0}}{\theta}+\frac{V_{\phi} \rho_{\phi} \phi_{t}}{\theta}+\widehat{B} \sqrt{\sigma_{\phi}} \varepsilon_{t+1}^{\phi}\right\}\right] \\
& =\delta\left(\mu_{c}+\frac{\varphi_{e}^{2} B^{2} \bar{\sigma}}{2 \theta}+\frac{\bar{\sigma}}{2 \theta}+V_{0}+V_{\phi} \rho_{\phi} \phi_{t}\right)+\delta \theta \log E_{t} \exp \left\{\log \left(1+A \rho_{\phi} \phi_{t}+A \sqrt{\sigma_{\phi}} \varepsilon_{t+1}^{\phi}\right)+\widehat{B} \sqrt{\sigma_{\phi}} \varepsilon_{t+1}^{\phi}\right\}(\mathrm{A} .27)
\end{aligned}
$$

where $A=\frac{0.8 \varphi_{e} B \sqrt{\bar{\sigma}}}{\theta}$ and $\widehat{B}=\frac{V_{\phi}}{\theta}$. Now we focus on the last term of the above equation.

$$
\begin{aligned}
& \log E_{t} \exp \left\{\log \left(1+A \rho_{\phi} \phi_{t}+A \sqrt{\sigma_{\phi}} \varepsilon_{t+1}^{\phi}\right)+\widehat{B} \sqrt{\sigma_{\phi}} \varepsilon_{t+1}^{\phi}\right\} \\
= & \log E_{t}\left[\left(1+A \rho_{\phi} \phi_{t}\right) \exp \left\{\widehat{B} \sqrt{\sigma_{\phi}} \varepsilon_{t+1}^{\phi}\right\}+A \sqrt{\sigma_{\phi}} \varepsilon_{t+1}^{\phi} \cdot \exp \left\{\widehat{B} \sqrt{\sigma_{\phi}} \varepsilon_{t+1}^{\phi}\right\}\right] \\
= & \log \left(\left(1+A \rho_{\phi} \phi_{t}\right) \exp \left\{\frac{\widehat{B}^{2} \sigma_{\phi}}{2}\right\}+E_{t}\left[A \sqrt{\sigma_{\phi}} \varepsilon_{t+1}^{\phi} \exp \left\{\widehat{B} \sqrt{\sigma_{\phi}} \varepsilon_{t+1}^{\phi}\right\}\right]\right)
\end{aligned}
$$

The conditional expectation term inside the logarithm is

$$
\begin{aligned}
E_{t}\left[A \sqrt{\sigma_{\phi}} \varepsilon_{t+1}^{\phi} \exp \left\{\widehat{B} \sqrt{\sigma_{\phi}} \varepsilon_{t+1}^{\phi}\right\}\right] & =\int_{-\infty}^{\infty} A \sqrt{\sigma_{\phi}} \varepsilon_{t+1}^{\phi} \exp \left\{\widehat{B} \sqrt{\sigma_{\phi}} \varepsilon_{t+1}^{\phi}\right\} \frac{1}{\sqrt{2 \pi}} \exp \left\{-\frac{\left(\varepsilon_{t+1}^{\phi}\right)^{2}}{2}\right\} d \varepsilon_{t+1}^{\phi} \\
& =\int_{-\infty}^{\infty} A \sqrt{\sigma_{\phi}} \varepsilon_{t+1}^{\phi} \frac{1}{\sqrt{2 \pi}} \exp \left\{-\frac{\left(\varepsilon_{t+1}^{\phi}-\widehat{B} \sqrt{\sigma_{\phi}}\right)^{2}}{2}\right\} \cdot \exp \left\{\frac{\widehat{B}^{2} \sigma_{\phi}}{2}\right\} d \varepsilon_{t+1}^{\phi} \\
\left(\text { Let } \widetilde{\varepsilon}=\varepsilon_{t+1}^{\phi}-\widehat{B} \sqrt{\sigma_{\phi}}\right) & =A \sqrt{\sigma_{\phi}} \exp \left\{\frac{\widehat{B}^{2} \sigma_{\phi}}{2}\right\} \int_{-\infty}^{\infty}\left(\widetilde{\varepsilon}+\widehat{B} \sqrt{\sigma_{\phi}}\right) \frac{1}{\sqrt{2 \pi}} \exp \left\{-\frac{\widetilde{\varepsilon}^{2}}{2}\right\} d \widetilde{\varepsilon} \\
& =A \sqrt{\sigma_{\phi}} \exp \left\{\frac{\widehat{B}^{2} \sigma_{\phi}}{2}\right\} \widehat{B} \sqrt{\sigma_{\phi}}
\end{aligned}
$$

Plugging the result into equation (A.28) and taking a Taylor expansion of logarithm about $\phi_{t}=0$, 
we get

$$
\begin{aligned}
& \log E_{t} \exp \left\{\log \left(1+A \rho_{\phi} \phi_{t}+A \sqrt{\sigma_{\phi}} \varepsilon_{t+1}^{\phi}\right)+\widehat{B} \sqrt{\sigma_{\phi}} \varepsilon_{t+1}^{\phi}\right\} \\
= & \log \left(\left(1+A \rho_{\phi} \phi_{t}\right) \exp \left\{\frac{\widehat{B}^{2} \sigma_{\phi}}{2}\right\}+A \sqrt{\sigma_{\phi}} \exp \left\{\frac{\widehat{B}^{2} \sigma_{\phi}}{2}\right\} \widehat{B} \sqrt{\sigma_{\phi}}\right) \\
= & \frac{\hat{B}^{2} \sigma_{\phi}}{2}+\log \left(1+A \rho_{\phi} \phi_{t}+A \widehat{B} \sigma_{\phi}\right) \approx \frac{\hat{B}^{2} \sigma_{\phi}}{2}+\log \left(1+A \widehat{B} \sigma_{\phi}\right)+\frac{A \rho_{\phi}}{1+A \hat{B} \sigma_{\phi}} \phi(\mathrm{A} .29)
\end{aligned}
$$

Now the value function $\widetilde{V}_{t}$ in equation (A.27) can be written as:

$$
\widetilde{V}_{t}=\delta\left(\mu_{c}+\frac{\varphi_{e}^{2} B^{2} \bar{\sigma}}{2 \theta}+\frac{\bar{\sigma}}{2 \theta}+V_{0}+V_{\phi} \rho_{\phi} \phi_{t}\right)+\delta \theta\left(\frac{\widehat{B}^{2} \sigma_{\phi}}{2}+\log \left(1+A \widehat{B} \sigma_{\phi}\right)+\frac{A \rho_{\phi}}{1+A \widehat{B} \sigma_{\phi}} \phi_{t}\right)
$$

Matching the coefficients of the value function:

$$
\begin{aligned}
V_{0} & =\frac{\delta}{1-\delta}\left[\mu_{c}+\frac{\left(\varphi_{e}^{2} B^{2}+1\right) \bar{\sigma}}{2 \theta}+\frac{\theta \hat{B}^{2} \sigma_{\phi}}{2}+\theta \log \left(1+A \hat{B} \sigma_{\phi}\right)\right] \\
V_{\phi} & =\frac{-\theta \pm \theta \sqrt{1+\frac{4 A^{2} \delta \rho_{\phi} \sigma_{\phi}}{1-\delta \rho_{\phi}}}}{2 A \sigma_{\phi}}
\end{aligned}
$$

For the two candidate solutions of $V_{\phi}$, only the positive solution satisfies our requirement. Notice that $\theta<0$ and $A=\frac{0.8 \varphi_{e} B \sqrt{\bar{\sigma}}}{\theta}<0$, then

$$
V_{\phi}=\frac{-\theta+\theta \sqrt{1+\frac{4 A^{2} \delta \rho_{\phi} \sigma_{\phi}}{1-\delta \rho_{\phi}}}}{2 A \sigma_{\phi}}
$$

Stochastic discount factor. The stochastic discount factor is obtained as the intertemporal marginal rate of substitution:

$m_{t+1}=\log \delta-\left(1-\frac{1}{\theta}\right) \Delta c_{t+1}+\frac{V_{0}}{\theta}+\frac{V_{\phi}}{\theta} \phi_{t+1}+\frac{B}{\theta} x_{t+1}-\log E_{t} \exp \left\{\frac{V_{0}+V_{\phi} \phi_{t+1}+B x_{t+1}+\mu_{c}+x_{t}+\sqrt{\bar{\sigma}} \varepsilon_{t+1}^{c}}{\theta}\right\}$ 
Note that the conditional distribution of $\varepsilon_{t+1}^{x}$ given $\varepsilon_{t+1}^{\phi}$ is skew normal $S K N\left(0,1, \nu_{t+1}\right), \varepsilon_{t+1}^{c}$ and $\varepsilon_{t+1}^{\phi}$ are $i . i . d$ normally distributed. We apply the law of iterated expectations to compute $m_{t+1}$ :

$$
\begin{aligned}
m_{t+1}= & \log \delta-\mu_{c}-x_{t}-\left(1-\frac{1}{\theta}\right) \sqrt{\bar{\sigma}} \varepsilon_{t+1}^{c}+\frac{V_{\phi}}{\theta} \sqrt{\sigma_{\phi}} \varepsilon_{t+1}^{\phi}+\frac{B}{\theta} \varphi_{e} \sqrt{\bar{\sigma}} \varepsilon_{t+1}^{x} \\
& -\log E_{t} E_{t}\left[\exp \left\{\frac{1}{\theta}\left(V_{\phi} \sqrt{\sigma_{\phi}} \varepsilon_{t+1}^{\phi}+B \varphi_{e} \sqrt{\bar{\sigma}} \varepsilon_{t+1}^{x}+\sqrt{\bar{\sigma}} \varepsilon_{t+1}^{c}\right)\right\} \mid \varepsilon_{t+1}^{\phi}\right] \\
= & \log \delta-\mu_{c}-x_{t}-\left(1-\frac{1}{\theta}\right) \sqrt{\sigma^{c}} \varepsilon_{t+1}^{c}+\frac{V_{\phi}}{\theta} \sqrt{\sigma_{\phi}} \varepsilon_{t+1}^{\phi}+\frac{B}{\theta} \varphi_{e} \sqrt{\bar{\sigma}} \varepsilon_{t+1}^{x}-\frac{\bar{\sigma}}{2 \theta^{2}} \\
& -\log E_{t}\left[\exp \left\{\frac{V_{\phi} \sqrt{\sigma_{\phi}}}{\theta} \varepsilon_{t+1}^{\phi}\right\} \cdot E_{t}\left(\exp \left\{\frac{B \varphi_{e} \sqrt{\bar{\sigma}}}{\theta} \varepsilon_{t+1}^{x}\right\} \mid \varepsilon_{t+1}^{\phi}\right)\right]
\end{aligned}
$$

We apply our approximation given in equation (A.26) to the last term of (A.31) again:

$$
\begin{aligned}
m_{t+1}= & \log \delta-\mu_{c}-x_{t}-\left(1-\frac{1}{\theta}\right) \sqrt{\bar{\sigma}} \varepsilon_{t+1}^{c}+\frac{V_{\phi}}{\theta} \sqrt{\sigma_{\phi}} \varepsilon_{t+1}^{\phi}+\frac{B}{\theta} \varphi_{e} \sqrt{\bar{\sigma}} \varepsilon_{t+1}^{x}-\frac{\bar{\sigma}}{2 \theta^{2}}-\frac{B^{2} \varphi_{e}^{2} \bar{\sigma}}{2 \theta^{2}} \\
& -\log E_{t} \exp \left\{\log \left(1+A \rho_{\phi} \phi_{t}+A \sqrt{\sigma_{\phi}} \varepsilon_{t+1}^{\phi}\right)+\widehat{B} \sqrt{\sigma_{\phi}} \varepsilon_{t+1}^{\phi}\right\}
\end{aligned}
$$

where $A=\frac{0.8 \varphi_{e} B \sqrt{\bar{\sigma}}}{\theta}$ and $\widehat{B}=\frac{V_{\phi}}{\theta}$ Using equation (A.28), the stochastic discount factor can be rewritten as:

$$
\begin{aligned}
m_{t+1}= & \log \delta-\mu_{c}-x_{t}-\frac{\bar{\sigma}}{2 \theta^{2}}-\frac{B^{2} \varphi_{e}^{2} \bar{\sigma}}{2 \theta^{2}}-\frac{\widehat{B}^{2} \sigma_{\phi}}{2}-\log \left(1+A \widehat{B} \sigma_{\phi}\right)-\frac{A \rho_{\phi}}{1+A \widehat{B} \sigma_{\phi}} \phi_{t} \\
& -\left(1-\frac{1}{\theta}\right) \sqrt{\bar{\sigma}} \varepsilon_{t+1}^{c}+\frac{V_{\phi}}{\theta} \sqrt{\sigma_{\phi}} \varepsilon_{t+1}^{\phi}+\frac{B}{\theta} \varphi_{e} \sqrt{\bar{\sigma}} \varepsilon_{t+1}^{x}
\end{aligned}
$$

Equity returns. We compute returns on a claim to the following dividend process:

$$
\Delta d_{t}=\lambda_{1} \mu_{c}+\lambda\left(x_{t-1}+\sqrt{\bar{\sigma}} \varepsilon_{t}^{c}\right)+\sqrt{\sigma_{d}} \sqrt{\bar{\sigma}} \varepsilon_{t}^{d}, \quad \varepsilon_{t}^{d} \sim N(0,1)
$$

As shown in section A.3 of the Technical Appendix, the log price-dividend ratio is

$$
v_{d, t}=\log E_{t} \exp \left\{m_{t+1}+\lambda_{1} \mu_{c}+\lambda\left(x_{t}+\sqrt{\bar{\sigma}} \varepsilon_{t+1}^{c}\right)+\sqrt{\sigma_{d}} \sqrt{\bar{\sigma}} \varepsilon_{t+1}^{d}+K_{0}+K_{1} v_{d, t+1}\right\}
$$

where $K_{1}=\frac{\exp \{\bar{v}\}}{1+\exp \{\bar{v}\}}$ and $K_{0}=\log (1+\exp \{\bar{v}\})-K_{1} \bar{v}$, and $\bar{v}$ denotes the unconditional mean of $v_{d, t}$. We guess that $v_{d, t}=v_{0}+v_{x} x_{t}+v_{\phi} \phi_{t}$. Then by plugging in the expression of $m_{t+1}$ given by 
equation (A.32), we have:

$$
\begin{aligned}
v_{d, t}= & \log E_{t} \exp \left\{m_{0}-x_{t}-m_{\phi} \phi_{t}-\sqrt{z_{c}} \varepsilon_{t+1}^{c}-\sqrt{z_{\phi}} \varepsilon_{t+1}^{\phi}-\sqrt{z_{x}} \varepsilon_{t+1}^{x}+\lambda_{1} \mu_{c}+\lambda\left(x_{t}+\sqrt{\bar{\sigma}} \varepsilon_{t+1}^{c}\right)\right. \\
& \left.+\sqrt{\sigma_{d}} \sqrt{\bar{\sigma}} \varepsilon_{t+1}^{d}+K_{0}+K_{1}\left(v_{0}+v_{x} x_{t+1}+v_{\phi} \phi_{t+1}\right)\right\} \\
= & \log E_{t} \exp \left\{m_{0}-(1-\lambda) x_{t}-m_{\phi} \phi_{t}-\left(\sqrt{z_{c}}-\lambda \sqrt{\bar{\sigma}}\right) \varepsilon_{t+1}^{c}-\sqrt{z_{\phi}} \varepsilon_{t+1}^{\phi}-\sqrt{z_{x}} \varepsilon_{t+1}^{x}+\lambda_{1} \mu_{c}+\sqrt{\sigma_{d}} \sqrt{\bar{\sigma}} \varepsilon_{t+1}^{d}\right. \\
& \left.+K_{0}+K_{1} v_{0}+K_{1} v_{x} \rho_{x} x_{t}+K_{1} v_{x} \varphi_{e} \sqrt{\bar{\sigma}} \varepsilon_{t+1}^{x}+K_{1} v_{\phi} \rho_{\phi} \phi_{t}+K_{1} v_{\phi} \sqrt{\sigma_{\phi}} \varepsilon_{t+1}^{\phi}\right\}
\end{aligned}
$$

where

$$
\begin{aligned}
& m_{0}=\log \delta-\mu_{c}-\frac{\bar{\sigma}}{2 \theta^{2}}-\frac{B^{2} \varphi_{e}^{2} \bar{\sigma}}{2 \theta^{2}}-\frac{\widehat{B}^{2} \sigma_{\phi}}{2}-\log \left(1+A \hat{B} \sigma_{\phi}\right), \quad m_{\phi}=\frac{A \rho_{\phi}}{1+A \widehat{B} \sigma_{\phi}} \\
& \sqrt{z_{c}}=\left(1-\frac{1}{\theta}\right) \sqrt{\bar{\sigma}}, \quad \sqrt{z_{\phi}}=-\frac{V_{\phi} \sqrt{\sigma_{\phi}}}{\theta}, \quad \sqrt{z_{x}}=-\frac{B \varphi_{e} \sqrt{\bar{\sigma}}}{\theta} .
\end{aligned}
$$

Again, we apply the law of iterated expectations to compute $v_{d, t}$ :

$$
\begin{aligned}
v_{d, t}= & m_{0}+\lambda_{1} \mu_{c}+K_{0}+K_{1} v_{0}+\frac{\left(\sqrt{z_{c}}-\lambda \sqrt{\bar{\sigma}}\right)^{2}}{2}+\frac{\sigma_{d} \bar{\sigma}}{2}+\left[K_{1} v_{x} \rho_{x}-(1-\lambda)\right] x_{t}+\left(K_{1} v_{\phi} \rho_{\phi}-m_{\phi}\right) \phi_{t} \\
& +\log E_{t} E_{t}\left[\exp \left\{\left(K_{1} v_{\phi} \sqrt{\sigma_{\phi}}-\sqrt{z_{\phi}}\right) \varepsilon_{t+1}^{\phi}+\left(K_{1} v_{x} \varphi_{e} \sqrt{\bar{\sigma}}-\sqrt{z_{x}}\right) \varepsilon_{t+1}^{x}\right\} \mid \varepsilon_{t+1}^{\phi}\right] \\
= & m_{0}+\lambda_{1} \mu_{c}+K_{0}+K_{1} v_{0}+\frac{\left(\sqrt{z_{c}}-\lambda \sqrt{\bar{\sigma}}\right)^{2}}{2}+\frac{\sigma_{d} \bar{\sigma}}{2}+\left[K_{1} v_{x} \rho_{x}-(1-\lambda)\right] x_{t}+\left(K_{1} v_{\phi} \rho_{\phi}-m_{\phi}\right) \phi_{t} \\
& +\frac{\left(\sqrt{z_{\phi}}-K_{1} v_{\phi} \sqrt{\sigma_{\phi}}\right)^{2}}{2}+\frac{\left(\sqrt{z_{x}}-K_{1} v_{x} \varphi_{e} \sqrt{\bar{\sigma}}\right)^{2}}{2} \\
& +\log E_{t} \exp \left\{\log \left(1+C \rho_{\phi} \phi_{t}+C \sqrt{\sigma_{\phi}} \varepsilon_{t+1}^{\phi}\right)+D \sqrt{\sigma_{\phi}} \varepsilon_{t+1}^{\phi}\right\}
\end{aligned}
$$

where $C=\sqrt{z_{x}}-K_{1} v_{x} \varphi_{e} \sqrt{\bar{\sigma}}=\left(\frac{B-K_{1} v_{x}}{\theta}\right) \varphi_{e} \sqrt{\bar{\sigma}}, D=\sqrt{z_{\phi}}-K_{1} v_{\phi} \sqrt{\sigma_{\phi}}=\left(\frac{V_{\phi}}{\theta}+K_{1} v_{\phi}\right)$. We apply our approximation given in equation (A.29) to the last term of (A.33) again:

$$
\begin{aligned}
v_{d, t}= & m_{0}+\lambda_{1} \mu_{c}+K_{0}+K_{1} v_{0}+\frac{\left(\sqrt{z_{c}}-\lambda \sqrt{\bar{\sigma}}\right)^{2}}{2}+\frac{\sigma_{d} \bar{\sigma}}{2}+\left[K_{1} v_{x} \rho_{x}-(1-\lambda)\right] x_{t}+\left(K_{1} v_{\phi} \rho_{\phi}-m_{\phi}\right) \phi_{t} \\
& +\frac{\left(\sqrt{z_{\phi}}-K_{1} v_{\phi} \sqrt{\sigma_{\phi}}\right)^{2}}{2}+\frac{\left(\sqrt{z_{x}}-K_{1} v_{x} \varphi_{e} \sqrt{\bar{\sigma}}\right)^{2}}{2}+\frac{D^{2} \sigma_{\phi}}{2}+\log \left(1+C D \sigma_{\phi}\right)+\frac{C \rho_{\phi}}{1+C D \sigma_{\phi}} \phi_{t}
\end{aligned}
$$


Matching the coefficients of $v_{d, t}$ :

$$
\begin{aligned}
v_{0}= & \frac{1}{1-K_{1}}\left\{m_{0}+\lambda_{1} \mu_{c}+K_{0}+\frac{\left(\sqrt{z_{c}}-\lambda \sqrt{\bar{\sigma}}\right)^{2}}{2}+\frac{\sigma_{d} \bar{\sigma}}{2}++\frac{\left(\sqrt{z_{\phi}}-K_{1} v_{\phi} \sqrt{\sigma_{\phi}}\right)^{2}}{2}+\frac{\left(\sqrt{z_{x}}-K_{1} v_{x} \varphi_{e} \sqrt{\bar{\sigma}}\right)^{2}}{2}\right. \\
& \left.+\frac{D^{2} \sigma_{\phi}}{2}+\log \left(1+C D \sigma_{\phi}\right)\right\} \\
v_{x}= & \frac{\lambda-1}{1-K_{1} \rho_{x}} \\
v_{\phi}= & \frac{1}{1-K_{1} \rho_{\phi}}\left(-\frac{A \rho_{\phi}}{1+A \widehat{B} \sigma_{\phi}}+\frac{C \rho_{\phi}}{1+C D \sigma_{\phi}}\right)
\end{aligned}
$$

Conditional Equity Returns. For the excess returns given in equation (A.9), the conditional expectation of the excess return is

$$
\begin{aligned}
E_{t}\left[\log R_{t+1}-r_{f, t}\right] & =\bar{r}^{e}+\left(K_{1} \rho_{\phi}-1\right) v_{\phi} \phi_{t}+K_{1} v_{x} \varphi_{e} \sqrt{\bar{\sigma}} \rho_{\phi} \sqrt{2 / \pi} \phi_{t} \\
& =\bar{r}^{e}+\left(\frac{A \rho_{\phi}}{1+A \widehat{B} \sigma_{\phi}}-\frac{C \rho_{\phi}}{1+C D \sigma_{\phi}}+K_{1} v_{x} \varphi_{e} \sqrt{\bar{\sigma}} \rho_{\phi} \sqrt{2 / \pi}\right) \phi_{t}
\end{aligned}
$$

where $\bar{r}^{e}=\log (1+\exp \{\bar{v}\})-\bar{v}+\log (\delta)-\left(\frac{1}{\theta}-\frac{1}{2}\right) \bar{\sigma}$.

\section{Second alternative analytical solution}

This solution differs from the previous ones in that we do not need to use Lew's approximationto solve for the utility function. The drawback is that the solution of the loadings of the state variables can only be computed up to a system of non-linear equations (see the system of equations in (A.36)).

Utility function. We shall solve for the Bellman equation (A.4) using the law of iterated expectations:

$$
\begin{aligned}
\widetilde{V}_{t} & =\delta \theta \log E_{t} E_{t}\left[\exp \left\{\frac{1}{\theta}\left(\mu_{c}+\varphi_{e} B \sqrt{\bar{\sigma}} \varepsilon_{t+1}^{x}+\sqrt{\bar{\sigma}} \varepsilon_{t+1}^{c}+\widetilde{V}_{t+1}\right)\right\} \mid \phi_{t+1}\right] \\
& =\delta \theta \log E_{t}\left[\exp \left\{\frac{\mu_{c}}{\theta}+\frac{\bar{\sigma}}{2 \theta^{2}}+\frac{V_{0}}{\theta}+\frac{V_{\phi}}{\theta} \phi_{t+1}\right\} \cdot E_{t}\left(\exp \left\{\frac{\varphi_{e} B \sqrt{\bar{\sigma}}}{\theta} \varepsilon_{t+1}^{x}\right\} \mid \phi_{t+1}\right)\right] \\
& =\delta \theta \log E_{t}\left[\exp \left\{\frac{\mu_{c}}{\theta}+\frac{\bar{\sigma}}{2 \theta^{2}}+\frac{V_{0}}{\theta}+\frac{V_{\phi}}{\theta} \phi_{t+1}+\log 2+\frac{\varphi_{e}^{2} B^{2} \bar{\sigma}}{2 \theta^{2}}+\log \Phi\left(\frac{\varphi_{e} B \sqrt{\bar{\sigma}}}{\theta} \phi_{t+1}\right)\right\}\right]
\end{aligned}
$$

Let $\widehat{A}=\frac{\varphi_{e} B \sqrt{\sigma}}{\theta}, \widehat{B}=\frac{V_{\phi}}{\theta}$. Since $\phi_{t}$ follows an $A R(1)$ process, that is $\phi_{t+1}=\rho_{\phi} \phi_{t}+\sqrt{\sigma_{\phi}} \varepsilon_{t+1}^{\phi}$, 
where $\varepsilon_{t+1}^{\phi} \sim N(0,1)$, the value function is:

$$
\begin{aligned}
\widetilde{V}_{t} & =\delta \theta \log E_{t} \exp \left\{\frac{\mu_{c}}{\theta}+\frac{\bar{\sigma}}{2 \theta^{2}}+\frac{V_{0}}{\theta}+\frac{V_{\phi}}{\theta}\left(\rho_{\phi} \phi_{t}+\sqrt{\sigma_{\phi}} \varepsilon_{t+1}^{\phi}\right)+\log (2)+\frac{\varphi_{e}^{2} B^{2} \bar{\sigma}}{2 \theta^{2}}+\log \Phi\left(A \rho_{\phi} \phi_{t}+A \sqrt{\sigma_{\phi}} \varepsilon_{t+1}^{\phi}\right)\right\} \\
& =\delta\left(\mu_{c}+\frac{\bar{\sigma}}{2 \theta}+V_{0}+V_{\phi} \rho_{\phi} \phi_{t}+\theta \log (2)+\frac{\varphi_{e}^{2} B^{2} \bar{\sigma}}{2 \theta}\right)+\delta \theta \log E_{t}\left[\exp \left\{\widehat{B} \sqrt{\sigma_{\phi}} \varepsilon_{t+1}^{\phi}\right\} \cdot \Phi\left(A \rho_{\phi} \phi_{t}+A \sqrt{\sigma_{\phi}} \varepsilon_{t+1}^{\phi}\right)\right]
\end{aligned}
$$

Let's focus on the conditional expectation inside the logarithm in the last term.

$$
\begin{aligned}
& E_{t}\left[\exp \left\{\widehat{B} \sqrt{\sigma_{\phi}} \varepsilon_{t+1}^{\phi}\right\} \cdot \Phi\left(A \rho_{\phi} \phi_{t}+A \sqrt{\sigma_{\phi}} \varepsilon_{t+1}^{\phi}\right)\right] \\
= & \int_{-\infty}^{\infty} \exp \left\{\widehat{B} \sqrt{\sigma_{\phi}} \varepsilon_{t+1}^{\phi}\right\} \cdot \Phi\left(A \rho_{\phi} \phi_{t}+A \sqrt{\sigma_{\phi}} \varepsilon_{t+1}^{\phi}\right) \frac{1}{\sqrt{2 \pi}} \exp \left\{-\frac{\left(\varepsilon_{t+1}^{\phi}\right)^{2}}{2}\right\} d \varepsilon_{t+1}^{\phi} \\
= & \int_{-\infty}^{\infty} \Phi\left(A \rho_{\phi} \phi_{t}+A \sqrt{\sigma_{\phi}} \varepsilon_{t+1}^{\phi}\right) \frac{1}{\sqrt{2 \pi}} \exp \left\{-\frac{\left(\varepsilon_{t+1}^{\phi}-\widehat{B} \sqrt{\sigma_{\phi}}\right)^{2}}{2}\right\} \exp \left\{\frac{\widehat{B}^{2} \sigma_{\phi}}{2}\right\} d \varepsilon_{t+1}^{\phi} \\
\left(\text { Let } \widetilde{\varepsilon}=\varepsilon_{t+1}^{\phi}-\widehat{B} \sqrt{\sigma_{\phi}}\right)= & \int_{-\infty}^{\infty} \Phi\left(A \rho_{\phi} \phi_{t}+A \sqrt{\sigma_{\phi}} \widehat{B} \sqrt{\sigma_{\phi}}+A \sqrt{\sigma_{\phi} \widetilde{\varepsilon}}\right) \frac{1}{\sqrt{2 \pi}} \exp \left\{-\frac{\widetilde{\varepsilon}^{2}}{2}\right\} \exp \left\{\frac{\widehat{B}^{2} \sigma_{\phi}}{2}\right\} d \widetilde{\varepsilon} \\
= & \exp \left\{\frac{\widehat{B}^{2} \sigma_{\phi}}{2}\right\} \cdot E\left[\Phi\left(A \rho_{\phi} \phi_{t}+A \widehat{B} \sigma_{\phi}+A \sqrt{\sigma_{\phi}} \widetilde{\varepsilon}\right)\right]
\end{aligned}
$$

Now use the following result from Zacks (1981). If $U$ is a $N(0,1)$ random variable, then

$$
E[\Phi(h U+k)]=\Phi\left(\frac{k}{\sqrt{1+h^{2}}}\right)
$$

for any real $h, k$ and where $\Phi(\cdot)$ denotes the cumulative distribution function of a $N(0,1)$ random variable. Then above conditional expectation is:

$$
E_{t}\left[\exp \left\{\widehat{B} \sqrt{\sigma_{\phi}} \varepsilon_{t+1}^{\phi}\right\} \cdot \Phi\left(A \rho_{\phi} \phi_{t}+A \sqrt{\sigma_{\phi}} \varepsilon_{t+1}^{\phi}\right)\right]=\exp \left\{\frac{\widehat{B}^{2} \sigma_{\phi}}{2}\right\} \Phi\left(\frac{A \rho_{\phi} \phi_{t}+A \widehat{B} \sigma_{\phi}}{\sqrt{1+A^{2} \sigma_{\phi}}}\right)
$$

Now we approximate $\log \Phi\left(\frac{A \rho_{\phi} \phi_{t}+A \widehat{B} \sigma_{\phi}}{1+A^{2} \sigma_{\phi}}\right)$ using the first order Taylor expansion of $\phi_{t}$ about 0 :

$$
\log E_{t}\left[\exp \left\{\widehat{B} \sqrt{\sigma_{\phi}} \varepsilon_{t+1}^{\phi}\right\} \cdot \Phi\left(A \rho_{\phi} \phi_{t}+A \sqrt{\sigma_{\phi}} \varepsilon_{t+1}^{\phi}\right)\right]=\frac{\widehat{B}^{2} \sigma_{\phi}}{2}+\log \Phi\left(\frac{A \widehat{B} \sigma_{\phi}}{\sqrt{1+A^{2} \sigma_{\phi}}}\right)
$$




$$
+\frac{\phi\left(\frac{A \widehat{B} \sigma_{\phi}}{\sqrt{1+A^{2} \sigma_{\phi}}}\right)}{\Phi\left(\frac{A \widehat{B} \sigma_{\phi}}{\sqrt{1+A^{2} \sigma_{\phi}}}\right)} \cdot \frac{A \rho_{\phi}}{\sqrt{1+A^{2} \sigma_{\phi}}} \cdot \phi_{t}
$$

Substituting this approximation into equation (A.34), the value function is approximately equal to

$$
\begin{aligned}
\widetilde{V}_{t} \approx & \delta\left(\mu_{c}+\frac{\bar{\sigma}}{2 \theta}+V_{0}+V_{\phi} \rho_{\phi} \phi_{t}+\theta \log (2)+\frac{\varphi_{e}^{2} B^{2} \bar{\sigma}}{2 \theta}+\frac{V_{\phi}^{2} \sigma_{\phi}}{\theta}\right)+ \\
& \delta \theta \log \Phi\left(\frac{A \widehat{B} \sigma_{\phi}}{\sqrt{1+A^{2} \sigma_{\phi}}}\right)+\delta \theta \frac{\phi\left(\frac{A \widehat{B} \sigma_{\phi}}{\sqrt{1+A^{2} \sigma_{\phi}}}\right)}{\Phi\left(\frac{A \widehat{B} \sigma_{\phi}}{\sqrt{1+A^{2} \sigma_{\phi}}}\right)} \cdot \frac{A \rho_{\phi} \phi_{t}}{\sqrt{1+A^{2} \sigma_{\phi}}}
\end{aligned}
$$

Matching the coefficients of the value function:

$$
\begin{aligned}
V_{0} & =\frac{\delta}{1-\delta}\left[\mu_{c}+\frac{\bar{\sigma}}{2 \theta}+V_{\phi} \rho_{\phi} \phi_{t}+\theta \log (2)+\frac{\varphi_{e}^{2} B^{2} \bar{\sigma}}{2 \theta}+\frac{V_{\phi}^{2} \sigma_{\phi}}{\theta}+\theta \log \Phi\left(\frac{A \widehat{B} \sigma_{\phi}}{\sqrt{1+A^{2} \sigma_{\phi}}}\right)\right] \\
V_{\phi} & =\delta \theta \frac{\phi\left(\frac{A \widehat{B} \sigma_{\phi}}{\sqrt{1+A^{2} \sigma_{\phi}}}\right)}{\Phi\left(\frac{A \widehat{B} \sigma_{\phi}}{\sqrt{1+A^{2} \sigma_{\phi}}}\right)} \cdot \frac{A \rho_{\phi}}{\sqrt{1+A^{2} \sigma_{\phi}}} .
\end{aligned}
$$

The preceding is a system of nonlinear equations since $\widehat{B}$ depends on $V_{\phi}$.

Stochastic discount factor. We compute the stochastic discount factor given in equation (A.31) using the law of iteration:

$$
\begin{aligned}
m_{t+1}= & \log \delta-\mu_{c}-x_{t}-\left(1-\frac{1}{\theta}\right) \sqrt{\sigma^{c}} \varepsilon_{t+1}^{c}+\frac{V_{\phi} \sqrt{\sigma_{\phi}}}{\theta} \varepsilon_{t+1}^{\phi}+\frac{\varphi_{e} B \sqrt{\bar{\sigma}}}{\theta} \varepsilon_{t+1}^{x}-\frac{\bar{\sigma}}{2 \theta^{2}} \\
& -\log E_{t}\left[\exp \left\{\frac{V_{\phi} \sqrt{\sigma_{\phi}}}{\theta} \varepsilon_{t+1}^{\phi}\right\} \cdot E_{t}\left(\exp \left\{\frac{\varphi_{e} B \sqrt{\bar{\sigma}}}{\theta} \varepsilon_{t+1}^{x}\right\} \mid \varepsilon_{t+1}^{\phi}\right)\right] \\
= & \log \delta-\mu_{c}-x_{t}-\left(1-\frac{1}{\theta}\right) \sqrt{\sigma^{c}} \varepsilon_{t+1}^{c}+\frac{V_{\phi} \sqrt{\sigma_{\phi}}}{\theta} \varepsilon_{t+1}^{\phi}+\frac{\varphi_{e} B \sqrt{\bar{\sigma}}}{\theta} \varepsilon_{t+1}^{x}-\frac{\bar{\sigma}}{2 \theta^{2}} \\
& -\log E_{t} \exp \left\{\frac{V_{\phi} \sqrt{\sigma_{\phi}}}{\theta} \varepsilon_{t+1}^{\phi}+\log (2)+\frac{\varphi_{e}^{2} B^{2} \bar{\sigma}}{2 \theta^{2}}+\log \Phi\left(\frac{\varphi_{e} B \sqrt{\bar{\sigma}}}{\theta} \phi_{t+1}\right)\right\} \\
= & \log \delta-\mu_{c}-x_{t}-\left(1-\frac{1}{\theta}\right) \sqrt{\sigma^{c}} \varepsilon_{t+1}^{c}+\frac{V_{\phi} \sqrt{\sigma_{\phi}}}{\theta} \varepsilon_{t+1}^{\phi}+\frac{\varphi_{e} B \sqrt{\bar{\sigma}}}{\theta} \varepsilon_{t+1}^{x}-\frac{\bar{\sigma}}{2 \theta^{2}}-\log (2)-\frac{\varphi_{e}^{2} B^{2} \bar{\sigma}}{2 \theta^{2}} \\
& -\log E_{t}\left[\exp \left\{\widehat{B} \sqrt{\sigma_{\phi}} \varepsilon_{t+1}^{\phi}\right\} \cdot \Phi\left(A \rho_{\phi} \phi_{t}+A \sqrt{\sigma_{\phi}} \varepsilon_{t+1}^{\phi}\right)\right]
\end{aligned}
$$


We apply the same approximation approach as in the computation of value function. Plugging equation (A.35) into above computation, the SDF is approximately equal to

$$
\begin{aligned}
m_{t+1}= & \log \delta-\mu_{c}-x_{t}-\left(1-\frac{1}{\theta}\right) \sqrt{\sigma^{c}} \varepsilon_{t+1}^{c}+\frac{V_{\phi} \sqrt{\sigma_{\phi}}}{\theta} \varepsilon_{t+1}^{\phi}+\frac{\varphi_{e} B \sqrt{\bar{\sigma}}}{\theta} \varepsilon_{t+1}^{x}-\frac{\bar{\sigma}}{2 \theta^{2}}-\log (2)-\frac{\varphi_{e}^{2} B^{2} \bar{\sigma}}{2 \theta^{2}} \\
& -\frac{\widehat{B}^{2} \sigma_{\phi}}{2}-\log \Phi\left(\frac{A \widehat{B} \sigma_{\phi}}{\sqrt{1+A^{2} \sigma_{\phi}}}\right)-\frac{\phi\left(\frac{A \widehat{B} \sigma_{\phi}}{\sqrt{1+A^{2} \sigma_{\phi}}}\right)}{\Phi\left(\frac{A \widehat{B} \sigma_{\phi}}{\sqrt{1+A^{2} \sigma_{\phi}}}\right)} \cdot \frac{A \rho_{\phi}}{\sqrt{1+A^{2} \sigma_{\phi}}} \cdot \phi_{t}
\end{aligned}
$$

Equity returns. We compute returns on a claim to the dividend process as in previous part A.14.1.

The log price-dividend ratio is

$$
v_{d, t}=\log E_{t} \exp \left\{m_{t+1}+\lambda_{1} \mu_{c}+\lambda\left(x_{t}+\sqrt{\bar{\sigma}} \varepsilon_{t+1}^{c}\right)+\sqrt{\sigma_{d}} \sqrt{\bar{\sigma}} \varepsilon_{t+1}^{d}+K_{0}+K_{1} v_{d, t+1}\right\}
$$

where $K_{1}=\frac{\exp \{\bar{v}\}}{1+\exp \{\bar{v}\}}$ and $K_{0}=\log (1+\exp \{\bar{v}\})-K_{1} \bar{v}$, and $\bar{v}$ denotes the unconditional mean of $v_{d, t}$. We guess that $v_{d, t}=v_{0}+v_{x} x_{t}+v_{\phi} \phi_{t}$. Then by plugging in the expression of $m_{t+1}$ given by equation (A.37), we have:

$$
\begin{aligned}
v_{d, t}= & \log E_{t} \exp \left\{m_{0}-(1-\lambda) x_{t}-m_{\phi} \phi_{t}-\left(\sqrt{z_{c}}-\lambda \sqrt{\bar{\sigma}}\right) \varepsilon_{t+1}^{c}-\sqrt{z_{\phi}} \varepsilon_{t+1}^{\phi}-\sqrt{z_{x}} \varepsilon_{t+1}^{x}\right. \\
& \left.+\lambda_{1} \mu_{c}+\sqrt{\sigma_{d}} \sqrt{\bar{\sigma}} \varepsilon_{t+1}^{d}+K_{0}+K_{1} v_{0}+K_{1} v_{x} \rho_{x} x_{t}+K_{1} v_{x} \varphi_{e} \sqrt{\bar{\sigma}} \varepsilon_{t+1}^{x}+K_{1} v_{\phi} \rho_{\phi} \phi_{t}+K_{1} v_{\phi} \sqrt{\sigma_{\phi}} \varepsilon_{t+1}^{\phi}\right\}
\end{aligned}
$$

where

$$
\begin{array}{r}
m_{0}=\log \delta-\mu_{c}-\frac{\bar{\sigma}}{2 \theta^{2}}-\frac{\varphi_{e}^{2} B^{2} \bar{\sigma}}{2 \theta^{2}}-\frac{\widehat{B}^{2} \sigma_{\phi}}{2}-\log \Phi\left(\frac{A \widehat{B} \sigma_{\phi}}{\sqrt{1+A^{2} \sigma_{\phi}}}\right) \\
m_{\phi}=\frac{\phi\left(\frac{A \widehat{B} \sigma_{\phi}}{\sqrt{1+A^{2} \sigma_{\phi}}}\right)}{\Phi\left(\frac{A \widehat{B} \sigma_{\phi}}{\sqrt{1+A^{2} \sigma_{\phi}}}\right)} \cdot \frac{A \rho_{\phi}}{\sqrt{1+A^{2} \sigma_{\phi}}}, \\
\sqrt{z_{c}}=\left(1-\frac{1}{\theta}\right) \sqrt{\bar{\sigma}}, \quad \sqrt{z_{\phi}}=-\frac{V_{\phi} \sqrt{\sigma_{\phi}}}{\theta}, \quad \sqrt{z_{x}}=-\frac{B \varphi_{e} \sqrt{\bar{\sigma}}}{\theta} .
\end{array}
$$

Again, we apply the law of iterated expectations to compute $v_{d, t}$ :

$$
\begin{aligned}
v_{d, t}= & m_{0}+\lambda_{1} \mu_{c}+K_{0}+K_{1} v_{0}+\frac{\left(\sqrt{z_{c}}-\lambda \sqrt{\bar{\sigma}}\right)^{2}}{2}+\frac{\sigma_{d} \bar{\sigma}}{2}+\left[K_{1} v_{x} \rho_{x}-(1-\lambda)\right] x_{t}+\left(K_{1} v_{\phi} \rho_{\phi}-m_{\phi}\right) \phi_{t} \\
& +\log E_{t} E_{t}\left[\exp \left\{\left(K_{1} v_{\phi} \sqrt{\sigma_{\phi}}-\sqrt{z_{\phi}}\right) \varepsilon_{t+1}^{\phi}+\left(K_{1} v_{x} \varphi_{e} \sqrt{\bar{\sigma}}-\sqrt{z_{x}}\right) \varepsilon_{t+1}^{x}\right\} \mid \varepsilon_{t+1}^{\phi}\right] \\
= & m_{0}+\lambda_{1} \mu_{c}+K_{0}+K_{1} v_{0}+\frac{\left(\sqrt{z_{c}}-\lambda \sqrt{\bar{\sigma}}\right)^{2}}{2}+\frac{\sigma_{d} \bar{\sigma}}{2}+\left[K_{1} v_{x} \rho_{x}-(1-\lambda)\right] x_{t}+\left(K_{1} v_{\phi} \rho_{\phi}-m_{\phi}\right) \phi_{t}
\end{aligned}
$$




$$
\begin{aligned}
& +\log E_{t}\left[\operatorname { e x p } \left\{\left(K_{1} v_{\phi} \sqrt{\sigma_{\phi}}-\sqrt{z_{\phi}}\right) \varepsilon_{t+1}^{\phi}+\log (2)+\frac{\left(K_{1} v_{x} \varphi_{e} \sqrt{\bar{\sigma}}-\sqrt{z_{x}}\right)^{2}}{2}+\right.\right. \\
& \left.\left.\log \Phi\left(\left(K_{1} v_{x} \varphi_{e} \sqrt{\bar{\sigma}}-\sqrt{z_{x}}\right) \phi_{t+1}\right)\right\}\right]
\end{aligned}
$$

Let $C=\sqrt{z_{x}}-K_{1} v_{x} \varphi_{e} \sqrt{\bar{\sigma}}=\left(\frac{B-K_{1} v_{x}}{\theta}\right) \varphi_{e} \sqrt{\bar{\sigma}}, D=\sqrt{z_{\phi}}-K_{1} v_{\phi} \sqrt{\sigma_{\phi}}=\left(\frac{V_{\phi}}{\theta}+K_{1} v_{\phi}\right)$.

Plugging the approximation equation (A.35) into above $v_{d, t}$ function, we get:

$$
\begin{aligned}
v_{d, t}= & m_{0}+\lambda_{1} \mu_{c}+K_{0}+K_{1} v_{0}+\frac{\left(\sqrt{z_{c}}-\lambda \sqrt{\bar{\sigma}}\right)^{2}}{2}+\frac{\sigma_{d} \bar{\sigma}}{2}+\log (2)+\left[K_{1} v_{x} \rho_{x}-(1-\lambda)\right] x_{t} \\
& +\left(K_{1} v_{\phi} \rho_{\phi}-m_{\phi}\right) \phi_{t}+\frac{\left(K_{1} v_{x} \varphi_{e} \sqrt{\bar{\sigma}}-\sqrt{z_{x}}\right)^{2}}{2}+\frac{\left(\sqrt{z_{\phi}}-K_{1} v_{\phi} \sqrt{\sigma_{\phi}}\right)^{2}}{2} \\
& +\log \Phi\left(\frac{C D \sqrt{\sigma_{\phi}}}{\sqrt{1+C^{2} \sigma_{\phi}}}\right)+\frac{\phi\left(\frac{C D \sqrt{\sigma_{\phi}}}{\sqrt{1+C^{2} \sigma_{\phi}}}\right)}{\Phi\left(\frac{C D \sqrt{\sigma_{\phi}}}{\sqrt{1+C^{2} \sigma_{\phi}}}\right)} \cdot \frac{C \rho_{\phi}}{\sqrt{1+C^{2} \sigma_{\phi}}} \cdot \phi_{t}
\end{aligned}
$$

Matching the coefficients of $v_{d, t}$ :

$$
\begin{aligned}
v_{0}= & \frac{1}{1-K_{1}}\left\{m_{0}+\lambda_{1} \mu_{c}+K_{0}+\frac{\left(\sqrt{z_{c}}-\lambda \sqrt{\bar{\sigma}}\right)^{2}}{2}+\frac{\sigma_{d} \bar{\sigma}}{2}+\frac{\left(\sqrt{z_{\phi}}-K_{1} v_{\phi} \sqrt{\sigma_{\phi}}\right)^{2}}{2}\right. \\
& \left.+\frac{\left(\sqrt{z_{x}}-K_{1} v_{x} \varphi_{e} \sqrt{\bar{\sigma}}\right)^{2}}{2}+\log (2)+\log \Phi\left(\frac{C D \sqrt{\sigma_{\phi}}}{\sqrt{1+C^{2} \sigma_{\phi}}}\right)\right\} \\
v_{x}= & \frac{\lambda-1}{1-K_{1} \rho_{x}} \\
v_{\phi}= & \frac{1}{1-K_{1} \rho_{\phi}}\left[-\frac{\phi\left(\frac{A \widehat{B} \sigma_{\phi}}{\sqrt{1+A^{2} \sigma_{\phi}}}\right)}{\Phi\left(\frac{A \widehat{B} \sigma_{\phi}}{\sqrt{1+A^{2} \sigma_{\phi}}}\right)} \cdot \frac{A \rho_{\phi}}{\sqrt{1+A^{2} \sigma_{\phi}}}+\frac{\phi\left(\frac{C D \sqrt{\sigma_{\phi}}}{\sqrt{1+C^{2} \sigma_{\phi}}}\right)}{\Phi\left(\frac{C D \sqrt{\sigma_{\phi}}}{\sqrt{1+C^{2} \sigma_{\phi}}}\right)} \cdot \frac{C \rho_{\phi}}{\sqrt{1+C^{2} \sigma_{\phi}}}\right]
\end{aligned}
$$


Table A.16: Comparison of Analytical Solutions for the Constant Volatility Case

\begin{tabular}{|c|c|c|c|}
\hline Coefficient & Paper Approximation & First Alternative & Second Alternative \\
\hline$\overline{V_{0}}$ & 0.9731 & 0.9838 & 0.9839 \\
\hline$V_{\phi}$ & 0.0039 & 0.0039 & 0.0039 \\
\hline$m_{0}$ & -0.0331 & -0.0325 & -0.0325 \\
\hline$m_{\phi}$ & -0.0247 & -0.0247 & -0.0246 \\
\hline$v_{0}$ & 4.2976 & 1.1025 & 1.1022 \\
\hline$v_{x}$ & 10.963 & 7.2615 & 7.2609 \\
\hline$v_{\phi}$ & 0.0135 & 0.0610 & 0.0552 \\
\hline $\begin{array}{l}\text { Loading of } \phi_{t} \text { on } \\
E_{t}\left[\log R_{t+1}-r_{f, t}\right]\end{array}$ & $-2.49 \times 10^{-5}$ & -0.0422 & -0.0377 \\
\hline
\end{tabular}

Notes - This table compares the analytical solutions for the constant volatility case with three alternative methods using the semi-annual calibration as given in Table 3.1. The column labeled "Paper Approximation" reports the analytical solution as shown in Section A.3 of the Technical Appendix. The column labeled "First Alternative" reports the analytical solution using an the method described in A.14.1. The column labeled "Second Alternative" reports the analytical solution using the method described in A.14.1.

Conditional Equity Returns. For the excess returns given in equation (A.9), the conditional expectation of the excess return is

$$
\begin{aligned}
& E_{t}\left[\log R_{t+1}-r_{f, t}\right]=\bar{r}^{e}+\left(K_{1} \rho_{\phi}-1\right) v_{\phi} \phi_{t}+K_{1} v_{x} \varphi_{e} \sqrt{\bar{\sigma}} \rho_{\phi} \sqrt{2 / \pi} \phi_{t} \\
= & \bar{r}^{e}+\left(\frac{\phi\left(\frac{A \widehat{B} \sigma_{\phi}}{\sqrt{1+A^{2} \sigma_{\phi}}}\right)}{\Phi\left(\frac{A \widehat{B} \sigma_{\phi}}{\sqrt{1+A^{2} \sigma_{\phi}}}\right)} \cdot \frac{A \rho_{\phi}}{\sqrt{1+A^{2} \sigma_{\phi}}}-\frac{\phi\left(\frac{C D \sqrt{\sigma_{\phi}}}{\sqrt{1+C^{2} \sigma_{\phi}}}\right)}{\Phi\left(\frac{C D \sqrt{\sigma_{\phi}}}{\sqrt{1+C^{2} \sigma_{\phi}}}\right)} \cdot \frac{C \rho_{\phi}}{\sqrt{1+C^{2} \sigma_{\phi}}}+K_{1} v_{x} \varphi_{e} \sqrt{\bar{\sigma}} \rho_{\phi} \sqrt{2 / \pi}\right) \phi_{t}
\end{aligned}
$$

where $\bar{r}^{e}=\log (1+\exp \{\bar{v}\})-\bar{v}+\log (\delta)-\left(\frac{1}{\theta}-\frac{1}{2}\right) \bar{\sigma}$.

\section{Comparison of analytical solutions}

Table A.16 compares the analytical solutions for the constant volatility case shown in Section A.3 of the Technical Appendix and two alternative approximations shown in this section using semiannual calibration as given in Table 3.1 in main text. The loadings of $\phi_{t}$ on both utility function $\left(V_{\phi}\right)$ and stochastic discount factor $\left(m_{\phi}\right)$ are very close. The coefficients $v_{0}, v_{x}$ and $v_{\phi}$ in the log-pricedividend ratio have the same signs although different magnitudes. The analytical solution used in main text generates smaller loading of $\phi_{t}$ on the log-price-dividend ratio and expected excess returns. Therefore, our analytical solution understates the role of skewness in the risk premium, and it can be considered as a conservative assessment. 


\section{Comparison of analytical and numerical solutions}

In this subsection, we compare the analytical and numerical solutions of the utility function $\widetilde{V}_{t}$ and $\log$ price-dividend ratio $v_{d, t}$ using a variety of monthly calibrations of $\phi_{t}$ process. Specifically, we alter the following three parameters that jointly determine the AR(1) process of $\phi_{t}$ in Markov transition approximation: the degree of persistence of the predictive component, $\rho_{\phi}$, the number of discrete grids $N$ for the symmetric state space $Z_{N}=\left\{z_{1}, \ldots, z_{N}\right\}$, and the range of the state space $[-\tilde{\psi}, \tilde{\psi}]$.

Table A.17 reports the discrepancies between the numerical and analytical solutions of $\widetilde{V}_{t}$ for the constant volatility case. The discrepancies are defined as the maximum absolute value of the ratio of the difference in the analytical solutions of value function $\widetilde{V}_{t}$ to the numerical solutions of $\widetilde{V}_{t}$. Specifically, we report the ratio of the exponentials of the value functions to avoid discontinuities in the discrepancy measure for values of $\widetilde{V}_{t}$ close to zero. In parenthesis, we report the conditional volatility of $\phi_{t}$ associated with $\rho_{\phi}$ and the state space $[-\tilde{\psi}, \tilde{\psi}]$, computed by $\sigma_{\phi}=\tilde{\psi}^{2}\left(1-\rho_{\phi}^{2}\right) /(N-1)$.

As shown, when we set $\rho_{\phi}=0.8$ and $\tilde{\psi}=0.97$ as a benchmark, the percentage error between the numerical and analytical solutions is only $0.052 \%$ with the number of discrete grids $N=5$. Moreover, when we increase the number of discrete grids, the discrepancy between these two solutions are smaller. This comparison demonstrates a good consistency of the analytical solution compared to the numerical one. Table A.18 reports the discrepancies between the numerical and analytical solutions of $v_{d, t}$ in the model with time-varying mean and skewness. The same conclusion can be drawn from this table.

\section{A.14.2 Time-varying volatility and skewness case}

In this subsection, we compare the analytical and numerical solutions of the value function $\widetilde{V}_{t}$ and $v_{d, t}$ for various calibrations of the parameters governing the dynamics of $\phi_{t}$. We modify the setup of the model presented in the previous section by replacing the constant variances (denoted as $\bar{\sigma}$ ) with the following time-varying variance process:

$$
\sigma_{t}=\bar{\sigma}\left(1-\rho_{\sigma}\right)+\rho_{\sigma} \sigma_{t-1}+\sqrt{\sigma_{\epsilon}} \varepsilon_{t}^{\sigma}
$$


Table A.17: Comparison of Analytical and Numerical Solutions of Utility Function for Constant Volatility Case

\begin{tabular}{|c|c|c|c|c|}
\hline \multicolumn{5}{|c|}{ PANEL A: $N=5$} \\
\hline \multirow[b]{2}{*}{$\widetilde{\psi}$} & \multicolumn{4}{|c|}{$\rho_{\phi}$} \\
\hline & 0.50 & 0.70 & 0.80 & 0.90 \\
\hline \multirow[t]{2}{*}{0.70} & 0.005 & 0.014 & 0.027 & 0.089 \\
\hline & $(0.092)$ & $(0.062)$ & $(0.044)$ & $(0.023)$ \\
\hline \multirow[t]{2}{*}{0.90} & 0.008 & 0.022 & 0.045 & 0.157 \\
\hline & $(0.152)$ & $(0.103)$ & $(0.073)$ & $(0.038)$ \\
\hline \multirow[t]{2}{*}{0.95} & 0.009 & 0.025 & 0.050 & 0.178 \\
\hline & $(0.169)$ & $(0.115)$ & $(0.081)$ & $(0.043)$ \\
\hline \multirow[t]{2}{*}{0.97} & 0.009 & 0.026 & 0.052 & 0.187 \\
\hline & $(0.176)$ & $(0.120)$ & $(0.085)$ & $(0.045)$ \\
\hline \multirow{2}{*}{0.99} & 0.009 & 0.027 & 0.055 & 0.196 \\
\hline & $(0.184)$ & $(0.125)$ & $(0.088)$ & $(0.047)$ \\
\hline \multicolumn{5}{|c|}{ PANEL B: $N=7$} \\
\hline & \multicolumn{4}{|c|}{$\rho_{\phi}$} \\
\hline$\widetilde{\psi}$ & 0.50 & 0.70 & 0.80 & 0.90 \\
\hline \multirow[t]{2}{*}{0.70} & 0.004 & 0.011 & 0.021 & 0.059 \\
\hline & $(0.061)$ & $(0.042)$ & $(0.029)$ & $(0.016)$ \\
\hline \multirow[t]{2}{*}{0.90} & 0.006 & 0.018 & 0.035 & 0.101 \\
\hline & $(0.101)$ & $(0.069)$ & $(0.049)$ & $(0.026)$ \\
\hline \multirow[t]{2}{*}{0.95} & 0.007 & 0.020 & 0.039 & 0.113 \\
\hline & $(0.113)$ & $(0.077)$ & $(0.054)$ & $(0.029)$ \\
\hline \multirow[t]{2}{*}{0.97} & 0.007 & 0.021 & 0.040 & 0.118 \\
\hline & $(0.118)$ & $(0.080)$ & $(0.056)$ & $(0.030)$ \\
\hline \multirow[t]{2}{*}{0.99} & 0.008 & 0.022 & 0.042 & 0.123 \\
\hline & $(0.123)$ & $(0.083)$ & $(0.059)$ & $(0.031)$ \\
\hline \multicolumn{5}{|c|}{ PANEL C: $N=9$} \\
\hline & \multicolumn{4}{|c|}{$\rho_{\phi}$} \\
\hline$\widetilde{\psi}$ & 0.50 & 0.70 & 0.80 & 0.90 \\
\hline \multirow[t]{2}{*}{0.70} & 0.003 & 0.009 & 0.017 & 0.045 \\
\hline & $(0.046)$ & $(0.031)$ & $(0.022)$ & $(0.012)$ \\
\hline \multirow[t]{2}{*}{0.90} & 0.005 & 0.015 & 0.028 & 0.075 \\
\hline & $(0.076)$ & $(0.052)$ & $(0.036)$ & $(0.019)$ \\
\hline \multirow[t]{2}{*}{0.95} & 0.006 & 0.017 & 0.031 & 0.084 \\
\hline & $(0.085)$ & $(0.058)$ & $(0.041)$ & $(0.021)$ \\
\hline \multirow[t]{2}{*}{0.97} & 0.006 & 0.017 & 0.032 & 0.088 \\
\hline & $(0.088)$ & $(0.060)$ & $(0.042)$ & $(0.022)$ \\
\hline \multirow[t]{2}{*}{0.99} & 0.006 & 0.018 & 0.034 & 0.092 \\
\hline & $(0.092)$ & $(0.062)$ & $(0.044)$ & $(0.023)$ \\
\hline
\end{tabular}

Notes - The table reports the maximum discrepancies for the case in which skewness is time-varying and variance is constant (see section A.3 of the Technical Appendix). The discrepancy is calculated as $\left[\exp \left(\left|\left(\widetilde{V}_{a}-\widetilde{V}_{m}\right)\right|\right)-1\right] \cdot 100$, where $\widetilde{V}_{a}$ denotes the analytical solution and $\widetilde{V}_{m}$ denotes the numerical solution of utility function with same monthly calibration. For numerical solution, $\phi_{t}$ process is generated using Markov approximation. The $\phi_{t}$ process are generated from the interval of the state space $[-\tilde{\psi}, \tilde{\psi}]$. The number in parentheses is the conditional volatility of $\phi_{t}$ process, computed by $\sigma_{\phi}=$ $\tilde{\psi}^{2}\left(1-\rho_{\phi}^{2}\right) /(N-1)$. 
Table A.19 reports the discrepancies between the numerical and analytical solutions of $\widetilde{V}_{t}$ in the model with time-varying mean and skewness. From the table, when $\rho_{\phi}=0.8$, as in our benchmark, the discrepancies between the numerical and analytical solutions are less than $4.652 \%$ for any value of the state space $[-\tilde{\psi}, \tilde{\psi}]$ and the number of discrete grid $N$. Even when we assume $\rho_{\phi}$ to be more persistent $\left(\rho_{\phi}=0.9\right)$, the discrepancies are still relatively small (the maximum discrepancy is 8.316 $\%$ ). Table A.20 reports the discrepancies between the numerical and analytical solutions of $v_{d, t}$. With $\rho_{\phi}=0.8$, the maximum discrepancy is only $1.046 \%$. In sum, we show that the analytical solution is reliable to be used to illustrate the role of key parameters in determining the value function, the log-ratio of price to dividend, and the expected excess return.

\section{A.15 Models with jumps}

This section considers two models with jumps in both the expected consumption growth rate, $x_{t}$, and in the time-varying volatility, $\sigma_{t}$, as in Drechsler and Yaron (2011). The dynamics of consumption growth is as follows:

$$
\begin{aligned}
\Delta c_{t+1} & =\mu_{c}+x_{t}+\sqrt{\sigma_{t}} \varepsilon_{t+1}^{c} \\
\Delta d_{t+1} & =\lambda \Delta c_{t+1}+\sqrt{\sigma_{d}} \sqrt{\sigma_{t}} \varepsilon_{t+1}^{d} \\
x_{t+1} & =\rho_{x} x_{t}+\varphi_{e} \sqrt{\sigma_{t}} \varepsilon_{t+1}^{x}+J_{t+1}^{x} \\
\sigma_{t+1} & =\left(1-\rho_{\sigma}\right) \bar{\sigma}+\rho_{\sigma} \sigma_{t}+\sqrt{\sigma_{\varepsilon}} \varepsilon_{t+1}^{\sigma}+J_{t+1}^{\sigma}
\end{aligned}
$$

where $\varepsilon_{t+1}^{c}, \varepsilon_{t+1}^{d}, \varepsilon_{t+1}^{x}$ and $\varepsilon_{t+1}^{\sigma}$ are $i . i . d$. distributed as standard normal, and $J_{t+1}^{x}$ and $J_{t+1}^{\sigma}$ are jump processes.

\section{A.15.1 General properties of jump processes}

The following two lemmas are useful in the derivation of equity returns in this model.

Lemma A.15.1. Let $N_{t+1} \sim \operatorname{Poisson}\left(\lambda_{t}\right), \xi_{j, t+1}^{x} \sim N\left(0, \sigma_{x}^{2}\right)$ i.i.d., and $\xi_{j, t+1}^{\sigma} \sim \operatorname{Exp}\left(\mu_{\sigma}\right)-\mu_{\sigma} i . i . d$ with mean $\mu_{\sigma}$. Let A be a scalar. Then:

$$
\log E_{t} \exp \left\{A \cdot \sum_{j=1}^{N_{t+1}} \xi_{j, t+1}^{x}\right\}=\lambda_{t}\left(\exp \left\{\frac{A^{2} \sigma_{x}^{2}}{2}\right\}-1\right)
$$




$$
\log E_{t} \exp \left\{A \cdot \sum_{j=1}^{N_{t+1}} \xi_{j, t+1}^{\sigma}\right\}=\lambda_{t}\left(\frac{\exp \left\{-A \mu_{\sigma}\right\}}{1-A \mu_{\sigma}}-1\right) \text {, when } A<\frac{1}{\mu_{\sigma}}
$$

Proof. For the Poisson-compounded jump in $x$ :

$$
\begin{aligned}
\log E_{t} \exp \left\{A \cdot \sum_{j=1}^{N_{t+1}} \xi_{j, t+1}^{x}\right\} & =\log E_{t}\left[E_{t}\left(\exp \left\{A \cdot \sum_{j=1}^{N_{t+1}} \xi_{j, t+1}^{x}\right\} \mid N_{t+1}\right)\right] \\
& =\log E_{t}\left[\left(E_{t} \exp \left\{A \cdot \xi_{j, t+1}^{x}\right\}\right)^{N_{t+1}}\right] \\
& =\log E_{t}\left[\left(\exp \left\{\frac{A^{2} \sigma_{x}^{2}}{2}\right\}\right)^{N_{t+1}}\right]
\end{aligned}
$$

Note that the moment-generating function of $N_{t+1} \sim \operatorname{Poisson}\left(\lambda_{t}\right)$ is $M_{N}(s)=E \exp \left\{s N_{t+1}\right\}=$ $\exp \left\{\lambda_{t}\left(e^{s}-1\right)\right\}$. Then

$$
\log E_{t} \exp \left\{A \cdot \sum_{j=1}^{N_{t+1}} \xi_{j, t+1}^{x}\right\}=\log M_{N}\left(\frac{A^{2} \sigma_{x}^{2}}{2}\right)=\lambda_{t}\left(\exp \left\{\frac{A^{2} \sigma_{x}^{2}}{2}\right\}-1\right)
$$

For the Poisson-compounded jump in $\sigma$ :

$$
\begin{aligned}
\log E_{t} \exp \left\{A \cdot \sum_{j=1}^{N_{t+1}} \xi_{j, t+1}^{\sigma}\right\} & =\log E_{t}\left[E_{t}\left(\exp \left\{A \cdot \sum_{j=1}^{N_{t+1}} \xi_{j, t+1}^{\sigma}\right\} \mid N_{t+1}\right)\right] \\
& =\log E_{t}\left[\left(E_{t} \exp \left\{A \cdot \xi_{j, t+1}^{\sigma}\right\}\right)^{N_{t+1}}\right]
\end{aligned}
$$

Note that the moment-generating function of $Z \sim \operatorname{Exp}\left(\mu_{\sigma}\right)$ with mean $\mu_{\sigma}$ is $M_{Z}(s)=E \exp \{s Z\}=$ $\frac{1}{1-s \mu_{\sigma}}$, when $s<\frac{1}{\mu_{\sigma}}$. Then for $\xi_{j, t+1}^{\sigma}=Z+\mu_{\sigma}, E_{t} \exp \left\{A \xi_{j, t+1}^{\sigma}\right\}=E_{t} \exp \left\{A Z-A \mu_{\sigma}\right\}=$ $\frac{\exp \left\{-A \mu_{\sigma}\right\}}{1-A \mu_{\sigma}}$, and therefore,

$$
\begin{aligned}
\log E_{t} \exp \left\{A \cdot \sum_{j=1}^{N_{t+1}} \xi_{j, t+1}^{\sigma}\right\} & =\log E_{t}\left[\left(\frac{\exp \left\{-A \mu_{\sigma}\right\}}{1-A \mu_{\sigma}}\right)^{N_{t+1}}\right] \\
& =\log M_{N}\left(\log \left(\frac{\exp \left\{-A \mu_{\sigma}\right\}}{1-A \mu_{\sigma}}\right)\right) \\
& =\lambda_{t}\left(\frac{\exp \left\{-A \mu_{\sigma}\right\}}{1-A \mu_{\sigma}}-1\right) .
\end{aligned}
$$


Lemma A.15.2. Let $N_{t+1} \sim$ Poisson $\left(\lambda_{t}\right), \xi_{j, t+1}^{x} \sim-\operatorname{Exp}\left(\mu_{x}\right)+\mu_{x}$ i.i.d.. Let A be a scalar. Then:

$$
\log E_{t} \exp \left\{A \cdot \sum_{j=1}^{N_{t+1}} \xi_{j, t+1}^{x}\right\}=\lambda_{t}\left(\frac{\exp \left\{A \mu_{x}\right\}}{1+A \mu_{x}}-1\right), \text { when } A>-\frac{1}{\mu_{x}}
$$

Proof. Note that the moment-generating function of $Z \sim \operatorname{Exp}\left(\mu_{x}\right)$ is $M_{Z}(s)=E \exp \{s Z\}=\frac{1}{1-s \mu_{x}}$, when $s<\frac{1}{\mu_{x}}$. Then for $\xi_{j, t+1}^{x}=-Z+\mu_{x}$,

$$
E_{t} \exp \left\{s \xi_{j, t+1}^{x}\right\}=E_{t} \exp \left\{s\left(-Z+\mu_{x}\right)\right\}=e^{s \mu_{x}} M_{Z}(-s)=\frac{\exp \left\{s \mu_{x}\right\}}{1+s \mu_{x}}
$$

Then, for the Poisson-compounded jump in $x$ :

$$
\begin{aligned}
\log E_{t} \exp \left\{A \cdot \sum_{j=1}^{N_{t+1}} \xi_{j, t+1}^{x}\right\} & =\log E_{t}\left[E_{t}\left(\exp \left\{A \cdot \sum_{j=1}^{N_{t+1}} \xi_{j, t+1}^{x}\right\} \mid N_{t+1}\right)\right] \\
& =\log E_{t}\left[\left(E_{t} \exp \left\{A \cdot \xi_{j, t+1}^{x}\right\}\right)^{N_{t+1}}\right] \\
& =\log E_{t}\left[\left(\frac{\exp \left\{A \mu_{x}\right\}}{1+A \mu_{x}}\right)^{N_{t+1}}\right], \text { when } A>-\frac{1}{\mu_{x}}
\end{aligned}
$$

Note that the moment-generating function of $N_{t+1} \sim \operatorname{Poisson}\left(\lambda_{t}\right)$ is $M_{N}(s)=E \exp \left\{s N_{t+1}\right\}=$ $\exp \left\{\lambda_{t}\left(e^{s}-1\right)\right\}$. Then

$$
\begin{aligned}
\log E_{t} \exp \left\{A \cdot \sum_{j=1}^{N_{t+1}} \xi_{j, t+1}^{x}\right\} & =\log M_{N}\left(\log \left(\frac{\exp \left\{A \mu_{x}\right\}}{1+A \mu_{x}}\right)\right) \\
& =\lambda_{t}\left(\frac{\exp \left\{A \mu_{x}\right\}}{1+A \mu_{x}}-1\right), \text { when } A>-\frac{1}{\mu_{x}}
\end{aligned}
$$

\section{A.15.2 Size of the jumps in $x_{t}$ have normal distribution}

We first consider the model in which jumps are compound-Poisson processes:

$$
J_{t+1}^{x}=\sum_{j=1}^{N_{t+1}^{x}} \xi_{j, t+1}^{x}, \text { where } N_{t+1}^{x} \sim \operatorname{Poisson}\left(l_{0}^{x}+l_{1}^{x} \sigma_{t}\right), \text { and } \xi_{j, t+1}^{x} \sim N\left(0, \sigma_{x}^{2}\right) \text { i.i.d. }
$$




$$
J_{t+1}^{\sigma}=\sum_{j=1}^{N_{t+1}^{\sigma}} \xi_{j, t+1}^{\sigma}, \text { where } N_{t+1}^{\sigma} \sim \operatorname{Poisson}\left(l_{0}^{\sigma}+l_{1}^{\sigma} \sigma_{t}\right) \text {, and } \xi_{j, t+1}^{\sigma} \sim \operatorname{Exp}\left(\mu_{\sigma}\right)-\mu_{\sigma} i . i . d \text {. }
$$

Utility function. We shall decompose value function $V_{t}$, defined in equation (A.1) as the sum of two terms: one is linear in $x_{t}$, the other one is linear in $\sigma_{t}$ :

$$
V_{t}=B x_{t}+\widetilde{V}\left(\sigma_{t}\right)
$$

we will use the notation $\widetilde{V}\left(\sigma_{t}\right)$ and $\widetilde{V}_{t}$ interchangeably. The value of $B$ is easy to obtain by plugging in the guess for $V_{t}$ and the process for $\Delta c_{t+1}$ in equation (A.1):

$$
\begin{aligned}
\widetilde{V}_{t}+B x_{t} & =\delta \theta \log E_{t} \exp \left\{\frac{\widetilde{V}_{t+1}+B x_{t+1}+\mu_{c}+x_{t}+\sqrt{\sigma_{t}} \varepsilon_{t+1}^{c}}{\theta}\right\} \\
& =\delta \theta \log E_{t} \exp \left\{\frac{\widetilde{V}_{t+1}+B \rho_{x} x_{t}+\varphi_{e} B \sqrt{\sigma_{t}} \varepsilon_{t+1}^{x}+B J_{t+1}^{x}+\mu_{c}+x_{t}+\sqrt{\sigma_{t}} \varepsilon_{t+1}^{c}}{\theta}\right\} \\
& =\delta\left(B \rho_{x}+1\right) x_{t}+\delta \theta \log E_{t} \exp \left\{\frac{\widetilde{V}_{t+1}+\varphi_{e} B \sqrt{\sigma_{t}} \varepsilon_{t+1}^{x}+B J_{t+1}^{x}+\mu_{c}+\sqrt{\sigma_{t}} \varepsilon_{t+1}^{c}}{\theta}\right\},
\end{aligned}
$$

from which it follows that

$$
B=\frac{\delta}{1-\delta \rho_{x}}
$$

The relevant recursion to solve for the remainder of the utility function then becomes

$$
\widetilde{V}_{t}=\delta \theta \log E_{t} \exp \left\{\frac{\mu_{c}+\varphi_{e} B \sqrt{\sigma_{t}} \varepsilon_{t+1}^{x}+B J_{t+1}^{x}+\sqrt{\sigma_{t}} \varepsilon_{t+1}^{c}+\widetilde{V}_{t+1}}{\theta}\right\} .
$$

We guess that the solution of this value function is a linear function of $\sigma_{t}$. That is $\widetilde{V}_{t}=V_{0}+V_{\sigma} \sigma_{t}$. By plugging our guess in the value function above, we get:

$$
\begin{aligned}
\widetilde{V}_{t}= & \delta \theta \log E_{t} \exp \left\{\frac{\mu_{c}+\varphi_{e} B \sqrt{\sigma_{t}} \varepsilon_{t+1}^{x}+B J_{t+1}^{x}+\sqrt{\sigma_{t}} \varepsilon_{t+1}^{c}+V_{0}+V_{\sigma}\left[\left(1-\rho_{\sigma}\right) \bar{\sigma}+\rho_{\sigma} \sigma_{t}+\sqrt{\sigma_{\varepsilon}} \varepsilon_{t+1}^{\sigma}+J_{t+1}^{\sigma}\right]}{\theta}\right\} \\
= & \delta \mu_{c}+\frac{\delta}{2 \theta}\left(B^{2} \varphi_{e}^{2}+1\right) \sigma_{t}+\delta V_{0}+\delta V_{\sigma}\left(1-\rho_{\sigma}\right) \bar{\sigma}+\delta V_{\sigma} \rho_{\sigma} \sigma_{t}+\frac{\delta}{2 \theta} V_{\sigma}^{2} \sigma_{\varepsilon} \\
& +\delta \theta\left(l_{0}^{x}+l_{1}^{x} \sigma_{t}\right)\left(\exp \left\{\frac{B^{2} \sigma_{x}^{2}}{2 \theta^{2}}\right\}-1\right)+\delta \theta\left(l_{0}^{\sigma}+l_{1}^{\sigma} \sigma_{t}\right)\left(\frac{\exp \left\{-V_{\sigma} \mu_{\sigma} / \theta\right\}}{1-V_{\sigma} \mu_{\sigma} / \theta}-1\right)
\end{aligned}
$$


Matching coefficient yields the following system of equations:

$$
\begin{aligned}
V_{0} & =\frac{1}{1-\delta}\left\{\delta \mu_{c}+\delta V_{\sigma}\left(1-\rho_{\sigma}\right) \bar{\sigma}+\frac{\delta}{2 \theta} V_{\sigma}^{2} \sigma_{\varepsilon}+\delta \theta l_{0}^{x}\left(\exp \left\{\frac{B^{2} \sigma_{x}^{2}}{2 \theta^{2}}\right\}-1\right)+\delta \theta l_{0}^{\sigma}\left(\frac{\exp \left\{-V_{\sigma} \mu_{\sigma} / \theta\right\}}{1-V_{\sigma} \mu_{\sigma} / \theta}-1\right)\right\} \\
V_{\sigma} & =\frac{1}{1-\delta \rho_{\sigma}}\left\{\frac{\delta}{2 \theta}\left(B^{2} \varphi_{e}^{2}+1\right)+\delta \theta l_{1}^{x}\left(\exp \left\{\frac{B^{2} \sigma_{x}^{2}}{2 \theta^{2}}\right\}-1\right)+\delta \theta l_{1}^{\sigma}\left(\frac{\exp \left\{-V_{\sigma} \mu_{\sigma} / \theta\right\}}{1-V_{\sigma} \mu_{\sigma} / \theta}-1\right)\right\}
\end{aligned}
$$

Stochastic discount factor. The stochastic discount factor is the intertemporal marginal rate of substitution as defined in equation A.3:

$$
\begin{aligned}
m_{t+1}= & \log \delta-\Delta c_{t+1}+\frac{V_{t+1}+\Delta c_{t+1}}{\theta}-\log E_{t} \exp \left\{\frac{V_{t+1}+\Delta c_{t+1}}{\theta}\right\} \\
= & \log \delta-\left(1-\frac{1}{\theta}\right)\left(\mu_{c}+x_{t}+\sqrt{\sigma_{t}} \varepsilon_{t+1}^{c}\right)+\frac{V_{0}}{\theta}+\frac{V_{\sigma}}{\theta}\left[\left(1-\rho_{\sigma}\right) \bar{\sigma}+\rho_{\sigma} \sigma_{t}+\sqrt{\sigma_{\varepsilon}} \varepsilon_{t+1}^{\sigma}+J_{t+1}^{\sigma}\right] \\
& +\frac{B}{\theta}\left(\rho_{x} x_{t}+\varphi_{e} \sqrt{\sigma_{t}} \varepsilon_{t+1}^{x}+J_{t+1}^{x}\right)-\log E_{t} \exp \left\{\frac { 1 } { \theta } \left[V_{0}+V_{\sigma}\left(1-\rho_{\sigma} \bar{\sigma}\right)+V_{\sigma} \rho_{\sigma} \sigma_{t}\right.\right. \\
& \left.\left.+V_{\sigma} \sqrt{\sigma_{\varepsilon}} \varepsilon_{t+1}^{\sigma}+V_{\sigma} J_{t+1}^{\sigma}+B \rho_{x} x_{t}+B \varphi_{e} \sqrt{\sigma_{t}} \varepsilon_{t+1}^{x}+B J_{t+1}^{x}+\mu_{c}+x_{t}+\sqrt{\sigma_{t}} \varepsilon_{t+1}^{c}\right]\right\} \\
= & \log \delta-\mu_{c}-x_{t}-\frac{V_{\sigma}^{2} \sigma_{\varepsilon}}{2 \theta^{2}}-\frac{B^{2} \varphi_{e}^{2}+1}{2 \theta^{2}} \sigma_{t}-\left(l_{0}^{x}+l_{1}^{x} \sigma_{t}\right)\left(\exp \left\{\frac{B^{2} \sigma_{x}^{2}}{2 \theta^{2}}\right\}-1\right)-\left(l_{0}^{\sigma}+l_{1}^{\sigma} \sigma_{t}\right) . \\
& \left(\frac{\exp \left\{-V_{\sigma} \mu_{\sigma} / \theta\right\}}{1-V_{\sigma} \mu_{\sigma} / \theta}-1\right)-\left(1-\frac{1}{\theta}\right) \sqrt{\sigma_{t}} \varepsilon_{t+1}^{c}+\frac{B}{\theta} \varphi_{e} \sqrt{\sigma_{t}} \varepsilon_{t+1}^{x}+\frac{V_{\sigma}}{\theta} \sqrt{\sigma_{\varepsilon}} \varepsilon_{t+1}^{\sigma}+\frac{B}{\theta} J_{t+1}^{x}+\frac{V_{\sigma}}{\theta} J_{t+1}^{\sigma}
\end{aligned}
$$

Note that the stochastic discount factor captures the Gaussian innovations and jumps in the conditional mean, in the conditional volatility.

Equity returns. Given the stochastic discount factor in (A.40), we compute $R_{t}$ on a claim to a dividend process with log-growth rate $\Delta d_{t}$ as follows:

$$
\Delta d_{t}=\lambda_{1} \mu_{c}+\lambda\left(x_{t-1}+\sqrt{\sigma_{t-1}} \varepsilon_{t}^{c}\right)+\sqrt{\sigma_{d}} \sqrt{\sigma_{t-1}} \varepsilon_{t}^{d}
$$

where the innovation $\varepsilon_{t+1}^{d}$ is $i . i . d$ distributed as a standard normal. The returns $R_{t+1}$ can be computed by solving the Euler equation

$$
E_{t}\left[\exp \left\{m_{t+1}\right\} \frac{1+V_{d, t+1}}{V_{d, t}} \exp \left\{\Delta d_{t+1}\right\}\right]=1
$$

where $R_{t+1}=\frac{1+V_{d, t+1}}{V_{d, t}} \exp \left\{\Delta d_{t+1}\right\}$ and $V_{d, t}$ is the price-dividend ratio. Following the same method 
as in previous sections,

$\log V_{d, t}=\log E_{t} \exp \left\{m_{t+1}+\lambda_{1} \mu_{c}+\lambda\left(x_{t}+\sqrt{\sigma_{t}} \varepsilon_{t+1}^{c}\right)+\sqrt{\sigma_{d}} \sqrt{\sigma_{t}} \varepsilon_{t+1}^{d}+K_{0}+K_{1} V_{d, t+1}\right\}$

where $K_{1}=\frac{\exp \{\bar{v}\}}{1+\exp \{\bar{v}\}}$ and $K_{0}=\log (1+\exp \{\bar{v}\})-K_{1} \bar{v}$. Guess that $\log V_{d, t}=v_{0}+v_{x} x_{t}+v_{\sigma} \sigma_{t}$.

Then,

$$
\begin{aligned}
& \log V_{d, t}=\log E_{t} \exp \left\{\log \delta-\mu_{c}-x_{t}-\frac{V_{\sigma}^{2} \sigma_{\varepsilon}}{2 \theta^{2}}-\frac{B^{2} \varphi_{e}^{2}+1}{2 \theta^{2}} \sigma_{t}-\left(l_{0}^{\sigma}+l_{1}^{\sigma} \sigma_{t}\right)\left(\frac{\exp \left\{-V_{\sigma} \mu_{\sigma} / \theta\right\}}{1-V_{\sigma} \mu_{\sigma} / \theta}-1\right)\right. \\
& -\left(l_{0}^{x}+l_{1}^{x} \sigma_{t}\right)\left(\exp \left\{\frac{B^{2} \sigma_{x}^{2}}{2 \theta^{2}}\right\}-1\right)-\left(1-\frac{1}{\theta}\right) \sqrt{\sigma_{t}} \varepsilon_{t+1}^{c}+\frac{B}{\theta} \varphi_{e} \sqrt{\sigma_{t}} \varepsilon_{t+1}^{x}+\frac{V_{\sigma}}{\theta} \sqrt{\sigma_{\varepsilon}} \varepsilon_{t+1}^{\sigma}+\frac{B}{\theta} J_{t+1}^{x} \\
& +\frac{V_{\sigma}}{\theta} J_{t+1}^{\sigma}+\lambda_{1} \mu_{c}+\lambda\left(x_{t}+\sqrt{\sigma_{t}} \varepsilon_{t+1}^{c}\right)+\sqrt{\sigma_{d}} \sqrt{\sigma_{t}} \varepsilon_{t+1}^{d}+K_{0}+K_{1}\left[v_{0}+v_{x}\left(\rho_{x} x_{t}+\varphi_{e} \sqrt{\sigma_{t}} \varepsilon_{t+1}^{x}+J_{t+1}^{x}\right)\right. \\
& \left.\left.+v_{\sigma}\left(\left(1-\rho_{\sigma}\right) \bar{\sigma}+\rho_{\sigma} \sigma_{t}+\sqrt{\sigma_{\varepsilon}} \varepsilon_{t+1}^{\sigma}+J_{t+1}^{\sigma}\right)\right]\right\} \\
& =\log \delta-\mu_{c}-x_{t}-\frac{V_{\sigma}^{2} \sigma_{\varepsilon}}{2 \theta^{2}}-\frac{B^{2} \varphi_{e}^{2}+1}{2 \theta^{2}} \sigma_{t}-\left(l_{0}^{\sigma}+l_{1}^{\sigma} \sigma_{t}\right)\left(\frac{\exp \left\{-V_{\sigma} \mu_{\sigma} / \theta\right\}}{1-V_{\sigma} \mu_{\sigma} / \theta}-1\right)-\left(l_{0}^{x}+l_{1}^{x} \sigma_{t}\right) . \\
& \left(\exp \left\{\frac{B^{2} \sigma_{x}^{2}}{2 \theta^{2}}\right\}-1\right)+\lambda_{1} \mu_{c}+\lambda x_{t}+K_{0}+K_{1} v_{0}+K_{1} v_{x} \rho_{x} x_{t}+K_{1} v_{\sigma}\left(1-\rho_{\sigma}\right) \bar{\sigma}+K_{1} v_{\sigma} \rho_{\sigma} \sigma_{t} \\
& +\log E_{t} \exp \left\{\left(\lambda-1+\frac{1}{\theta}\right) \sqrt{\sigma_{t}} \varepsilon_{t+1}^{c}+\sqrt{\sigma_{d}} \sqrt{\sigma_{t}} \varepsilon_{t+1}^{d}+\left(\frac{B}{\theta}+K_{1} v_{x}\right) \varphi_{e} \sqrt{\sigma_{t}} \varepsilon_{t+1}^{x}\right. \\
& \left.+\left(\frac{V_{\sigma}}{\theta}+K_{1} v_{\sigma}\right) \sqrt{\sigma_{\varepsilon}} \varepsilon_{t+1}^{\sigma}+\left(\frac{B}{\theta}+K_{1} v_{x}\right) J_{t+1}^{x}+\left(\frac{V_{\sigma}}{\theta}+K_{1} v_{\sigma}\right) J_{t+1}^{\sigma}\right\} \\
& =\log \delta-\mu_{c}-x_{t}-\frac{V_{\sigma}^{2} \sigma_{\varepsilon}}{2 \theta^{2}}-\frac{B^{2} \varphi_{e}^{2}+1}{2 \theta^{2}} \sigma_{t}-\left(l_{0}^{\sigma}+l_{1}^{\sigma} \sigma_{t}\right)\left(\frac{\exp \left\{-V_{\sigma} \mu_{\sigma} / \theta\right\}}{1-V_{\sigma} \mu_{\sigma} / \theta}-1\right)-\left(l_{0}^{x}+l_{1}^{x} \sigma_{t}\right) \text {. } \\
& \left(\exp \left\{\frac{B^{2} \sigma_{x}^{2}}{2 \theta^{2}}\right\}-1\right)+\lambda_{1} \mu_{c}+\lambda x_{t}+K_{0}+K_{1} v_{0}+K_{1} v_{x} \rho_{x} x_{t}+K_{1} v_{\sigma}\left(1-\rho_{\sigma}\right) \bar{\sigma}+K_{1} v_{\sigma} \rho_{\sigma} \sigma_{t} \\
& +\frac{1}{2}\left(\lambda-1+\frac{1}{\theta}\right)^{2} \sigma_{t}+\frac{1}{2} \sigma_{d} \sigma_{t}+\frac{1}{2}\left(\frac{B}{\theta}+K_{1} v_{x}\right)^{2} \varphi_{e}^{2} \sigma_{t}+\frac{1}{2}\left(\frac{V_{\sigma}}{\theta}+K_{1} v_{\sigma}\right)^{2} \sigma_{\varepsilon} \\
& +\left(l_{0}^{x}+l_{1}^{x} \sigma_{t}\right)\left[\exp \left\{\frac{1}{2}\left(\frac{B}{\theta}+K_{1} v_{x}\right)^{2} \sigma_{x}^{2}\right\}-1\right]+\left(l_{0}^{\sigma}+l_{1}^{\sigma} \sigma_{t}\right)\left[\frac{\exp \left\{-\left(V_{\sigma} / \theta+K_{1} v_{\sigma}\right) \mu_{\sigma}\right\}}{1-\left(V_{\sigma} / \theta+K_{1} v_{\sigma}\right) \mu_{\sigma}}-1\right]
\end{aligned}
$$

By matching coefficients, the $\log$ price-dividend ratio $\log V_{d, t}=v_{0}+v_{x} x_{t}+v_{\sigma} \sigma_{t}$, where

$$
\begin{aligned}
v_{0}= & \frac{1}{1-K_{1}}\left\{\log \delta+\left(\lambda_{1}-1\right) \mu_{c}+K_{0}+K_{1} v_{\sigma}\left(1-\rho_{\sigma}\right) \bar{\sigma}+\left(\frac{K_{1}^{2} v_{\sigma}^{2}}{2}+\frac{V_{\sigma} K_{1} v_{\sigma}}{\theta}\right) \sigma_{\varepsilon}\right. \\
& +l_{0}^{x}\left[\exp \left\{\frac{1}{2}\left(\frac{B}{\theta}+K_{1} v_{x}\right)^{2} \sigma_{x}^{2}\right\}-\exp \left\{\frac{B^{2} \sigma_{x}^{2}}{2 \theta^{2}}\right\}\right]+l_{0}^{\sigma}\left[\frac{\exp \left\{-\left(V_{\sigma} / \theta+K_{1} v_{\sigma}\right) \mu_{\sigma}\right\}}{1-\left(V_{\sigma} / \theta+K_{1} v_{\sigma}\right) \mu_{\sigma}}\right. \\
& \left.\left.-\frac{\exp \left\{-V_{\sigma} \mu_{\sigma} / \theta\right\}}{1-V_{\sigma} \mu_{\sigma} / \theta}\right]\right\} \\
v_{x}= & \frac{\lambda-1}{1-K_{1} \rho_{x}}
\end{aligned}
$$




$$
\begin{aligned}
v_{\sigma}= & \frac{1}{1-K_{1} \rho_{\sigma}}\left\{\frac{1}{2}\left(\lambda-1+\frac{1}{\theta}\right)^{2}-\frac{1}{2 \theta^{2}}+\frac{\sigma_{d}}{2}+\left(\frac{K_{1}^{2} v_{x}^{2}}{2}+\frac{B K_{1} v_{x}}{\theta}\right) \varphi_{e}^{2}\right. \\
& +l_{1}^{x}\left[\exp \left\{\frac{1}{2}\left(\frac{B}{\theta}+K_{1} v_{x}\right)^{2} \sigma_{x}^{2}\right\}-\exp \left\{\frac{B^{2} \sigma_{x}^{2}}{2 \theta^{2}}\right\}\right]+l_{1}^{\sigma}\left[\frac{\exp \left\{-\left(V_{\sigma} / \theta+K_{1} v_{\sigma}\right) \mu_{\sigma}\right\}}{1-\left(V_{\sigma} / \theta+K_{1} v_{\sigma}\right) \mu_{\sigma}}\right. \\
& \left.\left.-\frac{\exp \left\{-V_{\sigma} \mu_{\sigma} / \theta\right\}}{1-V_{\sigma} \mu_{\sigma} / \theta}\right]\right\}
\end{aligned}
$$

We can calculate the $\log R_{t+1}$ as

$$
\log R_{t+1}=\log \left(1+V_{d, t+1}\right)-\log V_{d, t}+\Delta d_{t+1} .
$$

Risk-free rate. The log-risk-free rate is computed as:

$$
\begin{aligned}
r_{f, t}= & -\log E_{t}\left[\exp \left\{m_{t+1}\right\}\right] \\
= & -\log \delta+\mu_{c}+x_{t}+\frac{V_{\sigma}^{2} \sigma_{\varepsilon}}{2 \theta^{2}}+\frac{B^{2} \varphi_{e}^{2}+1}{2 \theta^{2}} \sigma_{t}+\left(l_{0}^{\sigma}+l_{1}^{\sigma} \sigma_{t}\right)\left(\frac{\exp \left\{-V_{\sigma} \mu_{\sigma} / \theta\right\}}{1-V_{\sigma} \mu_{\sigma} / \theta}-1\right) \\
& +\left(l_{0}^{x}+l_{1}^{x} \sigma_{t}\right)\left(\exp \left\{\frac{B^{2} \sigma_{x}^{2}}{2 \theta^{2}}\right\}-1\right)-\log E_{t} \exp \left\{\left(1-\frac{1}{\theta}\right) \sqrt{\sigma_{t}} \varepsilon_{t+1}^{c}+\frac{B}{\theta} \varphi_{e} \sqrt{\sigma_{t}} \varepsilon_{t+1}^{x}\right. \\
& \left.+\frac{V_{\sigma}}{\theta} \sqrt{\sigma_{\varepsilon}} \varepsilon_{t+1}^{\sigma}+\frac{B}{\theta} J_{t+1}^{x}+\frac{V_{\sigma}}{\theta} J_{t+1}^{\sigma}\right\} \\
= & -\log \delta+\mu_{c}+x_{t}+\left(\frac{1}{\theta}-\frac{1}{2}\right) \sigma_{t} .
\end{aligned}
$$

\section{A.15.3 Size of jumps in $x_{t}$ has negative exponential distribution}

We modify the dynamics of the Poisson jump processes as follows:

$$
\begin{aligned}
J_{t+1}^{x} & =\sum_{j=1}^{N_{t+1}^{x}} \xi_{j, t+1}^{x}, \text { where } N_{t+1}^{x} \sim \operatorname{Poisson}\left(l_{0}^{x}+l_{1}^{x} \sigma_{t}\right), \text { and } \xi_{j, t+1}^{x} \sim-\operatorname{Exp}\left(\mu_{x}\right)+\mu_{x} i . i . d . \\
J_{t+1}^{\sigma}= & \sum_{j=1}^{N_{t+1}^{\sigma}} \xi_{j, t+1}^{\sigma}, \text { where } N_{t+1}^{\sigma} \sim \operatorname{Poisson}\left(l_{0}^{\sigma}+l_{1}^{\sigma} \sigma_{t}\right), \text { and } \xi_{j, t+1}^{\sigma} \sim \operatorname{Exp}\left(\mu_{\sigma}\right)-\mu_{\sigma} i . i . d .
\end{aligned}
$$


Utility function. Value function $V_{t}$, defined in equation (A.1) still equals $V_{t}=B x_{t}+\widetilde{V}\left(\sigma_{t}\right)$, where $B=\frac{\delta}{1-\delta \rho_{x}}$. We guess that the solution of the remainder of the utility function $\widetilde{V}_{t}$ is a linear function of $\sigma_{t}$. That is $\widetilde{V}_{t}=V_{0}+V_{\sigma} \sigma_{t}$. By plugging our guess in the value function (A.39), we get:

$$
\begin{aligned}
\widetilde{V}_{t}= & \delta \theta \log E_{t} \exp \left\{\frac{\mu_{c}+\varphi_{e} B \sqrt{\sigma_{t}} \varepsilon_{t+1}^{x}+B J_{t+1}^{x}+\sqrt{\sigma_{t}} \varepsilon_{t+1}^{c}+V_{0}+V_{\sigma}\left[\left(1-\rho_{\sigma}\right) \bar{\sigma}+\rho_{\sigma} \sigma_{t}+\sqrt{\sigma_{\varepsilon}} \varepsilon_{t+1}^{\sigma}+J_{t+1}^{\sigma}\right]}{\theta}\right\} \\
= & \delta \mu_{c}+\frac{\delta}{2 \theta}\left(B^{2} \varphi_{e}^{2}+1\right) \sigma_{t}+\delta V_{0}+\delta V_{\sigma}\left(1-\rho_{\sigma}\right) \bar{\sigma}+\delta V_{\sigma} \rho_{\sigma} \sigma_{t}+\frac{\delta}{2 \theta} V_{\sigma}^{2} \sigma_{\varepsilon}+\delta \theta\left(l_{0}^{\sigma}+l_{1}^{\sigma} \sigma_{t}\right) . \\
& \left(\frac{\exp \left\{-V_{\sigma} \mu_{\sigma} / \theta\right\}}{1-V_{\sigma} \mu_{\sigma} / \theta}\right)+\delta \theta\left(l_{0}^{x}+l_{1}^{x} \sigma_{t}\right)\left(\frac{\exp \left\{B \mu_{x} / \theta\right\}}{1+B \mu_{x} / \theta}-1\right)
\end{aligned}
$$

Matching coefficient yields the following system of equations:

$$
\begin{aligned}
V_{0} & =\frac{1}{1-\delta}\left\{\delta \mu_{c}+\delta V_{\sigma}\left(1-\rho_{\sigma}\right) \bar{\sigma}+\frac{\delta}{2 \theta} V_{\sigma}^{2} \sigma_{\varepsilon}+\delta \theta l_{0}^{x}\left(\frac{\exp \left\{B \mu_{x} / \theta\right\}}{1+B \mu_{x} / \theta}-1\right)+\delta \theta l_{0}^{\sigma}\left(\frac{\exp \left\{-V_{\sigma} \mu_{\sigma} / \theta\right\}}{1-V_{\sigma} \mu_{\sigma} / \theta}-1\right)\right\} \\
V_{\sigma} & =\frac{1}{1-\delta \rho_{\sigma}}\left\{\frac{\delta}{2 \theta}\left(B^{2} \varphi_{e}^{2}+1\right)+\delta \theta l_{1}^{x}\left(\frac{\exp \left\{B \mu_{x} / \theta\right\}}{1+B \mu_{x} / \theta}-1\right)+\delta \theta l_{1}^{\sigma}\left(\frac{\exp \left\{-V_{\sigma} \mu_{\sigma} / \theta\right\}}{1-V_{\sigma} \mu_{\sigma} / \theta}-1\right)\right\}
\end{aligned}
$$

Stochastic discount factor. The stochastic discount factor is the intertemporal marginal rate of substitution as defined in equation A.3:

$$
\begin{aligned}
m_{t+1}= & \log \delta-\left(1-\frac{1}{\theta}\right)\left(\mu_{c}+x_{t}+\sqrt{\sigma_{t}} \varepsilon_{t+1}^{c}\right)+\frac{V_{0}}{\theta}+\frac{V_{\sigma}}{\theta}\left[\left(1-\rho_{\sigma}\right) \bar{\sigma}+\rho_{\sigma} \sigma_{t}+\sqrt{\sigma_{\varepsilon}} \varepsilon_{t+1}^{\sigma}+J_{t+1}^{\sigma}\right] \\
& +\frac{B}{\theta}\left(\rho_{x} x_{t}+\varphi_{e} \sqrt{\sigma_{t}} \varepsilon_{t+1}^{x}+J_{t+1}^{x}\right)-\log E_{t} \exp \left\{\frac { 1 } { \theta } \left[V_{0}+V_{\sigma}\left(1-\rho_{\sigma} \bar{\sigma}\right)+V_{\sigma} \rho_{\sigma} \sigma_{t}\right.\right. \\
& \left.\left.+V_{\sigma} \sqrt{\sigma_{\varepsilon}} \varepsilon_{t+1}^{\sigma}+V_{\sigma} J_{t+1}^{\sigma}+B \rho_{x} x_{t}+B \varphi_{e} \sqrt{\sigma_{t}} \varepsilon_{t+1}^{x}+B J_{t+1}^{x}+\mu_{c}+x_{t}+\sqrt{\sigma_{t}} \varepsilon_{t+1}^{c}\right]\right\} \\
= & \log \delta-\mu_{c}-x_{t}-\frac{V_{\sigma}^{2} \sigma_{\varepsilon}}{2 \theta^{2}}-\frac{B^{2} \varphi_{e}^{2}+1}{2 \theta^{2}} \sigma_{t}-\left(l_{0}^{x}+l_{1}^{x} \sigma_{t}\right)\left(\frac{\exp \left\{B \mu_{x} / \theta\right\}}{1+B \mu_{x} / \theta}-1\right) \\
& -\left(l_{0}^{\sigma}+l_{1}^{\sigma} \sigma_{t}\right)\left(\frac{\exp \left\{-V_{\sigma} \mu_{\sigma} / \theta\right\}}{1-V_{\sigma} \mu_{\sigma} / \theta}-1\right)-\left(1-\frac{1}{\theta}\right) \sqrt{\sigma_{t}} \varepsilon_{t+1}^{c}+\frac{B}{\theta} \varphi_{e} \sqrt{\sigma_{t}} \varepsilon_{t+1}^{x} \\
& +\frac{V_{\sigma}}{\theta} \sqrt{\sigma_{\varepsilon}} \varepsilon_{t+1}^{\sigma}+\frac{B}{\theta} J_{t+1}^{x}+\frac{V_{\sigma}}{\theta} J_{t+1}^{\sigma}
\end{aligned}
$$

Equity returns. Given the stochastic discount factor in (A.41), the returns $R_{t+1}$ can be computed by solving the Euler equation

$$
E_{t}\left[\exp \left\{m_{t+1}\right\} \frac{1+V_{d, t+1}}{V_{d, t}} \exp \left\{\Delta d_{t+1}\right\}\right]=1
$$

where $R_{t+1}=\frac{1+V_{d, t+1}}{V_{d, t}} \exp \left\{\Delta d_{t+1}\right\}$ and $V_{d, t}$ is the price-dividend ratio. Following the same method 
as in previous sections, we guess that $\log V_{d, t}=v_{0}+v_{x} x_{t}+v_{\sigma} \sigma_{t}$. Then,

$$
\begin{aligned}
\log V_{d, t}= & \log E_{t} \exp \left\{m_{t+1}+\lambda_{1} \mu_{c}+\lambda\left(x_{t}+\sqrt{\sigma_{t}} \varepsilon_{t+1}^{c}\right)+\sqrt{\sigma_{d}} \sqrt{\sigma_{t}} \varepsilon_{t+1}^{d}+K_{0}+K_{1} V_{d, t+1}\right\} \\
= & \log \delta-\mu_{c}-x_{t}-\frac{V_{\sigma}^{2} \sigma_{\varepsilon}}{2 \theta^{2}}-\frac{B^{2} \varphi_{e}^{2}+1}{2 \theta^{2}} \sigma_{t}-\left(l_{0}^{x}+l_{1}^{x} \sigma_{t}\right)\left(\frac{\exp \left\{B \mu_{x} / \theta\right\}}{1+B \mu_{x} / \theta}-1\right) \\
& -\left(l_{0}^{\sigma}+l_{1}^{\sigma} \sigma_{t}\right)\left(\frac{\exp \left\{-V_{\sigma} \mu_{\sigma} / \theta\right\}}{1-V_{\sigma} \mu_{\sigma} / \theta}-1\right)+\lambda_{1} \mu_{c}+\lambda x_{t}+K_{0}+K_{1} v_{0}+K_{1} v_{x} \rho_{x} x_{t}+K_{1} v_{\sigma}\left(1-\rho_{\sigma}\right) \bar{\sigma} \\
& +K_{1} v_{\sigma} \rho_{\sigma} \sigma_{t}+\frac{1}{2}\left(\lambda-1+\frac{1}{\theta}\right)^{2} \sigma_{t}+\frac{1}{2} \sigma_{d} \sigma_{t}+\frac{1}{2}\left(\frac{B}{\theta}+K_{1} v_{x}\right)^{2} \varphi_{e}^{2} \sigma_{t}+\frac{1}{2}\left(\frac{V_{\sigma}}{\theta}+K_{1} v_{\sigma}\right)^{2} \sigma_{\varepsilon} \\
& +\left(l_{0}^{x}+l_{1}^{x} \sigma_{t}\right)\left(\frac{\exp \left\{\left(B / \theta+K_{1} v_{x}\right) \mu_{x}\right\}}{1+\left(B / \theta+K_{1} v_{x}\right) \mu_{x}}-1\right)+\left(l_{0}^{\sigma}+l_{1}^{\sigma} \sigma_{t}\right)\left[\frac{\exp \left\{-\left(V_{\sigma} / \theta+K_{1} v_{\sigma}\right) \mu_{\sigma}\right\}}{1-\left(V_{\sigma} / \theta+K_{1} v_{\sigma}\right) \mu_{\sigma}}-1\right]
\end{aligned}
$$

where $K_{1}=\frac{\exp \{\bar{v}\}}{1+\exp \{\bar{v}\}}$ and $K_{0}=\log (1+\exp \{\bar{v}\})-K_{1} \bar{v}$.

By matching coefficients,

$$
\begin{aligned}
v_{0}= & \frac{1}{1-K_{1}}\left\{\log \delta+\left(\lambda_{1}-1\right) \mu_{c}+K_{0}+K_{1} v_{\sigma}\left(1-\rho_{\sigma}\right) \bar{\sigma}+\left(\frac{K_{1}^{2} v_{\sigma}^{2}}{2}+\frac{V_{\sigma} K_{1} v_{\sigma}}{\theta}\right) \sigma_{\varepsilon}\right. \\
& +l_{0}^{x}\left[\frac{\exp \left\{\left(B / \theta+K_{1} v_{x}\right) \mu_{x}\right\}}{1+\left(B / \theta+K_{1} v_{x}\right) \mu_{x}}-\frac{\exp \left\{B \mu_{x} / \theta\right\}}{1+B \mu_{x} / \theta}\right] \\
& \left.+l_{0}^{\sigma}\left[\frac{\exp \left\{-\left(V_{\sigma} / \theta+K_{1} v_{\sigma}\right) \mu_{\sigma}\right\}}{1-\left(V_{\sigma} / \theta+K_{1} v_{\sigma}\right) \mu_{\sigma}}-\frac{\exp \left\{-V_{\sigma} \mu_{\sigma} / \theta\right\}}{1-V_{\sigma} \mu_{\sigma} / \theta}\right]\right\} \\
v_{x}= & \frac{\lambda-1}{1-K_{1} \rho_{x}} \\
v_{\sigma}= & \frac{1}{1-K_{1} \rho_{\sigma}}\left\{\frac{1}{2}\left(\lambda-1+\frac{1}{\theta}\right)^{2}-\frac{1}{2 \theta^{2}}+\frac{\sigma_{d}}{2}+\left(\frac{K_{1}^{2} v_{x}^{2}}{2}+\frac{B K_{1} v_{x}}{\theta}\right) \varphi_{e}^{2}\right. \\
& +l_{1}^{x}\left[\frac{\exp \left\{\left(B / \theta+K_{1} v_{x}\right) \mu_{x}\right\}}{1+\left(B / \theta+K_{1} v_{x}\right) \mu_{x}}-\frac{\exp \left\{B \mu_{x} / \theta\right\}}{1+B \mu_{x} / \theta}\right] \\
& \left.+l_{1}^{\sigma}\left[\frac{\exp \left\{-\left(V_{\sigma} / \theta+K_{1} v_{\sigma}\right) \mu_{\sigma}\right\}}{1-\left(V_{\sigma} / \theta+K_{1} v_{\sigma}\right) \mu_{\sigma}}-\frac{\exp \left\{-V_{\sigma} \mu_{\sigma} / \theta\right\}}{1-V_{\sigma} \mu_{\sigma} / \theta}\right]\right\}
\end{aligned}
$$

Risk-free rate. The log-risk-free rate is computed as:

$$
r_{f, t}=-\log E_{t}\left[\exp \left\{m_{t+1}\right\}\right]=-\log \delta+\mu_{c}+x_{t}+\left(\frac{1}{\theta}-\frac{1}{2}\right) \sigma_{t}
$$

\section{A.15.4 Sensitivity analysis}

Tables A.21 reports the sensitivity of some key asset pricing moments to alternative calibrations. They document that the moments are in line with what reported in Table 4.2 in main text. 
Table A.18: Comparison of Analytical and Numerical Solutions of Log Price-Dividend Ratio for Constant Volatility Case

\begin{tabular}{|c|c|c|c|c|}
\hline \multicolumn{5}{|c|}{ PANEL A: $N=5$} \\
\hline \multirow[b]{2}{*}{$\widetilde{\psi}$} & \multicolumn{4}{|c|}{$\rho_{\phi}$} \\
\hline & 0.50 & 0.70 & 0.80 & 0.90 \\
\hline \multirow[t]{2}{*}{0.7} & 0.033 & 0.124 & 0.2821 & 0.9812 \\
\hline & $(0.092)$ & $(0.062)$ & $(0.044)$ & $(0.023)$ \\
\hline \multirow[t]{2}{*}{0.9} & 0.065 & 0.246 & 0.5571 & 1.9112 \\
\hline & $(0.152)$ & $(0.103)$ & $(0.073)$ & $(0.038)$ \\
\hline \multirow[t]{2}{*}{0.95} & 0.075 & 0.285 & 0.6441 & 2.198 \\
\hline & $(0.169)$ & $(0.115)$ & $(0.081)$ & $(0.043)$ \\
\hline \multirow[t]{2}{*}{0.97} & 0.079 & 0.301 & 0.6811 & 2.319 \\
\hline & $(0.176)$ & $(0.120)$ & $(0.085)$ & $(0.045)$ \\
\hline \multirow[t]{2}{*}{0.99} & 0.084 & 0.318 & 0.7201 & 2.443 \\
\hline & $(0.184)$ & $(0.125)$ & $(0.088)$ & $(0.047)$ \\
\hline \multicolumn{5}{|c|}{ PANEL B: $N=7$} \\
\hline & \multicolumn{4}{|c|}{$\rho_{\phi}$} \\
\hline$\widetilde{\psi}$ & 0.50 & 0.70 & 0.80 & 0.90 \\
\hline \multirow[t]{2}{*}{0.7} & 0.029 & 0.109 & 0.2511 & 0.911 \\
\hline & $(0.061)$ & $(0.042)$ & $(0.029)$ & $(0.016)$ \\
\hline \multirow[t]{2}{*}{0.9} & 0.057 & 0.219 & 0.5031 & 1.813 \\
\hline & $(0.101)$ & $(0.069)$ & $(0.049)$ & $(0.026)$ \\
\hline \multirow[t]{2}{*}{0.95} & 0.066 & 0.254 & 0.5841 & 2.099 \\
\hline & $(0.113)$ & $(0.077)$ & $(0.054)$ & $(0.029)$ \\
\hline \multirow[t]{2}{*}{0.97} & 0.070 & 0.269 & 0.6191 & 2.219 \\
\hline & $(0.118)$ & $(0.080)$ & $(0.056)$ & $(0.030)$ \\
\hline \multirow[t]{2}{*}{0.99} & 0.074 & 0.285 & 0.6541 & 2.344 \\
\hline & $(0.123)$ & $(0.083)$ & $(0.059)$ & $(0.031)$ \\
\hline \multicolumn{5}{|c|}{ PANEL C: $N=9$} \\
\hline & \multicolumn{4}{|c|}{$\rho_{\phi}$} \\
\hline$\widetilde{\psi}$ & 0.50 & 0.70 & 0.80 & 0.90 \\
\hline \multirow[t]{2}{*}{0.7} & 0.022 & 0.079 & 0.1751 & 0.605 \\
\hline & $(0.046)$ & $(0.031)$ & $(0.022)$ & $(0.012)$ \\
\hline \multirow[t]{2}{*}{0.9} & 0.042 & 0.153 & 0.3411 & 1.189 \\
\hline & $(0.076)$ & $(0.052)$ & $(0.036)$ & $(0.019)$ \\
\hline \multirow[t]{2}{*}{0.95} & 0.048 & 0.177 & 0.3941 & 1.374 \\
\hline & $(0.085)$ & $(0.058)$ & $(0.041)$ & $(0.021)$ \\
\hline \multirow[t]{2}{*}{0.97} & 0.051 & 0.187 & 0.4171 & 1.452 \\
\hline & $(0.088)$ & $(0.060)$ & $(0.042)$ & $(0.022)$ \\
\hline \multirow[t]{2}{*}{0.99} & 0.054 & 0.197 & 0.4401 & 1.533 \\
\hline & $(0.092)$ & $(0.062)$ & $(0.044)$ & $(0.023)$ \\
\hline
\end{tabular}

Notes - The table reports the maximum discrepancies for the case in which skewness is time-varying and variance is constant (see section A.3 of the Technical Appendix). The discrepancy is calculated as $\left(\exp \left(\left|\left(v_{d, a}-v_{d, m}\right)\right|\right)-1\right) \cdot 100$, where $v_{d, a}$ denotes the analytical solution and $v_{d, m}$ denotes the numerical solution of log price-dividend ratio with same monthly calibration. For numerical solution, $\phi_{t}$ process is generated using Markov approximation. The number in parentheses is the conditional volatility of $\phi_{t}$ process, computed by $\sigma_{\phi}=\tilde{\psi}^{2}\left(1-\rho_{\phi}^{2}\right) /(N-1)$. 
Table A.19: Comparison of Analytical and Numerical Solutions of Utility Function in the Model with Time-Varying Volatility and Skewness

\begin{tabular}{|c|c|c|c|c|}
\hline \multicolumn{5}{|c|}{ PANEL A: $N=5$} \\
\hline \multirow[b]{2}{*}{$\widetilde{\psi}$} & \multicolumn{4}{|c|}{$\rho_{\phi}$} \\
\hline & 0.50 & 0.70 & 0.80 & 0.90 \\
\hline \multirow[t]{2}{*}{0.7} & 0.644 & 1.390 & 2.1741 & 3.838 \\
\hline & $(0.092)$ & $(0.062)$ & $(0.044)$ & $(0.023)$ \\
\hline \multirow[t]{2}{*}{0.9} & 0.830 & 1.794 & 2.8051 & 4.925 \\
\hline & $(0.152)$ & $(0.103)$ & $(0.073)$ & $(0.038)$ \\
\hline \multirow[t]{2}{*}{0.95} & 0.877 & 1.895 & 2.9631 & 5.193 \\
\hline & $(0.169)$ & $(0.115)$ & $(0.081)$ & $(0.043)$ \\
\hline \multirow[t]{2}{*}{0.97} & 0.895 & 1.936 & 3.0261 & 5.300 \\
\hline & $(0.176)$ & $(0.120)$ & $(0.085)$ & $(0.045)$ \\
\hline \multirow{2}{*}{0.99} & 0.914 & 1.976 & 3.0891 & 5.407 \\
\hline & $(0.184)$ & $(0.125)$ & $(0.088)$ & $(0.047)$ \\
\hline \multicolumn{5}{|c|}{ PANEL B: $N=7$} \\
\hline & \multicolumn{4}{|c|}{$\rho_{\phi}$} \\
\hline$\widetilde{\psi}$ & 0.50 & 0.70 & 0.80 & 0.90 \\
\hline \multirow[t]{2}{*}{0.7} & 0.856 & 1.852 & 2.9021 & 5.163 \\
\hline & $(0.061)$ & $(0.042)$ & $(0.029)$ & $(0.016)$ \\
\hline \multirow[t]{2}{*}{0.9} & 1.104 & 2.390 & 3.7481 & 6.664 \\
\hline & $(0.101)$ & $(0.069)$ & $(0.049)$ & $(0.026)$ \\
\hline \multirow[t]{2}{*}{0.95} & 1.166 & 2.525 & 3.9611 & 7.039 \\
\hline & $(0.113)$ & $(0.077)$ & $(0.054)$ & $(0.029)$ \\
\hline \multirow[t]{2}{*}{0.97} & 1.191 & 2.579 & 4.0461 & 7.189 \\
\hline & $(0.118)$ & $(0.080)$ & $(0.056)$ & $(0.030)$ \\
\hline \multirow[t]{2}{*}{0.99} & 1.216 & 2.633 & 4.1311 & 7.339 \\
\hline & $(0.123)$ & $(0.083)$ & $(0.059)$ & $(0.031)$ \\
\hline \multicolumn{5}{|c|}{ PANEL C: $N=9$} \\
\hline & \multicolumn{4}{|c|}{$\rho_{\phi}$} \\
\hline$\widetilde{\psi}$ & 0.50 & 0.70 & 0.80 & 0.90 \\
\hline \multirow[t]{2}{*}{0.7} & 0.963 & 2.082 & 3.2651 & 5.829 \\
\hline & $(0.046)$ & $(0.031)$ & $(0.022)$ & $(0.012)$ \\
\hline \multirow[t]{2}{*}{0.9} & 1.241 & 2.687 & 4.2201 & 7.541 \\
\hline & $(0.076)$ & $(0.052)$ & $(0.036)$ & $(0.019)$ \\
\hline \multirow[t]{2}{*}{0.95} & 1.310 & 2.839 & 4.4601 & 7.971 \\
\hline & $(0.085)$ & $(0.058)$ & $(0.041)$ & $(0.021)$ \\
\hline \multirow[t]{2}{*}{0.97} & 1.338 & 2.900 & 4.5561 & 8.143 \\
\hline & $(0.088)$ & $(0.060)$ & $(0.042)$ & $(0.022)$ \\
\hline \multirow[t]{2}{*}{0.99} & 1.366 & 2.961 & 4.6521 & 8.316 \\
\hline & $(0.092)$ & $(0.062)$ & $(0.044)$ & $(0.023)$ \\
\hline
\end{tabular}

Notes - The table reports the maximum discrepancies for the case in which both variance and skewness are time-varying (see section A.11 of the Technical Appendix). The discrepancy is calculated as $\left[\exp \left(\left|\left(\widetilde{V}_{a}-\widetilde{V}_{m}\right)\right|\right)-1\right] \cdot 100$, where $\widetilde{V}_{a}$ denotes the analytical solution and $\widetilde{V}_{m}$ denotes the numerical solution of utility function with same monthly calibration. For numerical solution, $\phi_{t}$ process is generated using Markov approximation. The $\phi_{t}$ process are generated from the interval of the state space $[-\tilde{\psi}, \tilde{\psi}]$. The number in parentheses is the conditional volatility of $\phi_{t}$ process, computed by $\sigma_{\phi}=$ $\tilde{\psi}^{2}\left(1-\rho_{\phi}^{2}\right) /(N-1)$. 
Table A.20: Comparison of Analytical and Numerical Solutions of Log Price-Dividend Ratio in the Model with Time-Varying Volatility and Skewness

\begin{tabular}{|c|c|c|c|c|}
\hline \multicolumn{5}{|c|}{ PANEL A: $N=5$} \\
\hline \multirow[b]{2}{*}{$\widetilde{\psi}$} & \multicolumn{4}{|c|}{$\rho_{\phi}$} \\
\hline & 0.50 & 0.70 & 0.80 & 0.90 \\
\hline \multirow[t]{2}{*}{0.7} & 0.065 & 0.218 & 0.4301 & 1.604 \\
\hline & $(0.092)$ & $(0.062)$ & $(0.044)$ & $(0.023)$ \\
\hline \multirow[t]{2}{*}{0.9} & 0.106 & 0.339 & 0.6451 & 2.396 \\
\hline & $(0.152)$ & $(0.103)$ & $(0.073)$ & $(0.038)$ \\
\hline \multirow[t]{2}{*}{0.95} & 0.119 & 0.377 & 0.7121 & 2.609 \\
\hline & $(0.169)$ & $(0.115)$ & $(0.081)$ & $(0.043)$ \\
\hline \multirow[t]{2}{*}{0.97} & 0.124 & 0.394 & 0.7411 & 2.694 \\
\hline & $(0.176)$ & $(0.120)$ & $(0.085)$ & $(0.045)$ \\
\hline \multirow{2}{*}{0.99} & 0.130 & 0.411 & 0.7711 & 2.781 \\
\hline & $(0.184)$ & $(0.125)$ & $(0.088)$ & $(0.047)$ \\
\hline \multicolumn{5}{|c|}{ PANEL B: $N=7$} \\
\hline & \multicolumn{4}{|c|}{$\rho_{\phi}$} \\
\hline$\widetilde{\psi}$ & 0.50 & 0.70 & 0.80 & 0.90 \\
\hline \multirow[t]{2}{*}{0.7} & 0.072 & 0.290 & 0.6501 & 1.781 \\
\hline & $(0.061)$ & $(0.042)$ & $(0.029)$ & $(0.016)$ \\
\hline \multirow[t]{2}{*}{0.9} & 0.098 & 0.389 & 0.8631 & 2.289 \\
\hline & $(0.101)$ & $(0.069)$ & $(0.049)$ & $(0.026)$ \\
\hline \multirow[t]{2}{*}{0.95} & 0.105 & 0.416 & 0.9191 & 2.414 \\
\hline & $(0.113)$ & $(0.077)$ & $(0.054)$ & $(0.029)$ \\
\hline \multirow[t]{2}{*}{0.97} & 0.108 & 0.426 & 0.9411 & 2.464 \\
\hline & $(0.118)$ & $(0.080)$ & $(0.056)$ & $(0.030)$ \\
\hline \multirow[t]{2}{*}{0.99} & 0.111 & 0.437 & 0.9641 & 2.513 \\
\hline & $(0.123)$ & $(0.083)$ & $(0.059)$ & $(0.031)$ \\
\hline \multicolumn{5}{|c|}{ PANEL C: $N=9$} \\
\hline & \multicolumn{4}{|c|}{$\rho_{\phi}$} \\
\hline$\widetilde{\psi}$ & 0.50 & 0.70 & 0.80 & 0.90 \\
\hline \multirow[t]{2}{*}{0.7} & 0.077 & 0.315 & 0.7131 & 1.993 \\
\hline & $(0.046)$ & $(0.031)$ & $(0.022)$ & $(0.012)$ \\
\hline \multirow[t]{2}{*}{0.9} & 0.104 & 0.419 & 0.9401 & 2.567 \\
\hline & $(0.076)$ & $(0.052)$ & $(0.036)$ & (0.019) \\
\hline \multirow[t]{2}{*}{0.95} & 0.111 & 0.446 & 0.9991 & 2.710 \\
\hline & $(0.085)$ & $(0.058)$ & $(0.041)$ & $(0.021)$ \\
\hline \multirow[t]{2}{*}{0.97} & 0.114 & 0.457 & 1.0231 & 2.767 \\
\hline & $(0.088)$ & $(0.060)$ & $(0.042)$ & $(0.022)$ \\
\hline \multirow[t]{2}{*}{0.99} & 0.117 & 0.469 & 1.0461 & 2.824 \\
\hline & $(0.092)$ & $(0.062)$ & $(0.044)$ & $(0.023)$ \\
\hline
\end{tabular}

Notes - The table reports the maximum discrepancies for the case in which both variance and skewness are time-varying (see section A.11 of the Technical Appendix). The discrepancy is calculated as $\left(\exp \left(\left|\left(v_{d, a}-v_{d, m}\right)\right|\right)-1\right) \cdot 100$, where $v_{d, a}$ denotes the analytical solution and $v_{d, m}$ denotes the numerical solution of log price-dividend ratio with same monthly calibration. For numerical solution, $\phi_{t}$ process is generated using Markov approximation. The number in parentheses is the conditional volatility of $\phi_{t}$ process, computed by $\sigma_{\phi}=\tilde{\psi}^{2}\left(1-\rho_{\phi}^{2}\right) /(N-1)$. 
Table A.21: Sensitivity Analysis: Model with Jumps

\begin{tabular}{|c|c|c|c|c|c|c|}
\hline \multicolumn{7}{|c|}{ Panel A:Autoregressive coefficient of the expected consumption growth rate $\rho_{x}$} \\
\hline & \multicolumn{2}{|c|}{$\rho_{x}=0.955$} & \multicolumn{2}{|c|}{$\rho_{x}=0.965$} & \multicolumn{2}{|c|}{$\rho_{x}=0.970$} \\
\hline & {$[1]$} & {$[2]$} & {$[1]$} & {$[2]$} & [1] & {$[2]$} \\
\hline$E\left[r_{t}^{d}-r_{t}^{f}\right]$ & $\begin{array}{c}3.34 \\
{[0.27,6.41]}\end{array}$ & $\begin{array}{c}3.67 \\
{[0.71,6.63]}\end{array}$ & $\begin{array}{c}5.18 \\
{[1.95,8.41]}\end{array}$ & $\begin{array}{c}6.12 \\
{[3.02,9.22]}\end{array}$ & $\begin{array}{c}7.43 \\
{[4.11,10.76]}\end{array}$ & $\begin{array}{c}10.40 \\
{[7.27,13.54]}\end{array}$ \\
\hline$\sigma\left[r_{t}^{d}-r_{t}^{f}\right]$ & $\begin{array}{c}15.74 \\
{[12.29,19.18]}\end{array}$ & $\begin{array}{c}15.72 \\
{[12.15,19.29]}\end{array}$ & $\begin{array}{c}16.97 \\
{[13.04,20.90]}\end{array}$ & $\begin{array}{c}17.07 \\
{[12.83,21.32]}\end{array}$ & $\begin{array}{c}18.13 \\
{[13.48,22.77]}\end{array}$ & $\begin{array}{c}18.89 \\
{[13.13,24.65]}\end{array}$ \\
\hline$E\left[r_{t}^{f}\right]$ & $\begin{array}{c}2.15 \\
{[1.37,2.94]}\end{array}$ & $\begin{array}{c}2.15 \\
{[1.35,2.94]}\end{array}$ & $\begin{array}{c}2.16 \\
{[1.16,3.15]}\end{array}$ & $\begin{array}{c}2.15 \\
{[1.14,3.15]}\end{array}$ & $\begin{array}{c}2.16 \\
{[1.01,3.31]}\end{array}$ & $\begin{array}{c}2.14 \\
{[0.98,3.31]}\end{array}$ \\
\hline$\sigma\left[r_{t}^{f}\right]$ & $\begin{array}{c}1.88 \\
{[1.28,2.47]}\end{array}$ & $\begin{array}{c}1.87 \\
{[1.23,2.50]}\end{array}$ & $\begin{array}{c}2.13 \\
{[1.44,2.82]}\end{array}$ & $\begin{array}{c}2.11 \\
{[1.38,2.85]}\end{array}$ & $\begin{array}{c}2.29 \\
{[1.53,3.06]}\end{array}$ & $\begin{array}{c}2.28 \\
{[1.47,3.09]} \\
\end{array}$ \\
\hline \multicolumn{7}{|c|}{ Panel B: Jump intensity in $\sigma_{t}$} \\
\hline & \multicolumn{2}{|c|}{$l_{1}^{\sigma}=0.6 / 12 / \bar{\sigma}$} & \multicolumn{2}{|c|}{$l_{1}^{\sigma}=1 / 12 / \bar{\sigma}$} & \multicolumn{2}{|c|}{$l_{1}^{\sigma}=1.4 / 12 / \bar{\sigma}$} \\
\hline & [1] & {$[2]$} & {$[1]$} & {$[2]$} & [1] & {$[2]$} \\
\hline$\overline{E\left[r_{t}^{d}-r_{t}^{f}\right]}$ & $\begin{array}{c}3.22 \\
{[0.26,6.18]}\end{array}$ & $\begin{array}{c}3.55 \\
{[0.60,6.51]}\end{array}$ & $\begin{array}{c}3.51 \\
{[0.38,6.64]}\end{array}$ & $\begin{array}{c}3.89 \\
{[0.84,6.94]}\end{array}$ & $\begin{array}{c}3.95 \\
{[0.76,7.15]}\end{array}$ & $\begin{array}{c}4.45 \\
1.33,7.57\end{array}$ \\
\hline$\sigma\left[r_{t}^{d}-r_{t}^{f}\right]$ & $\begin{array}{c}15.42 \\
{[12.31,18.54]}\end{array}$ & $\begin{array}{c}15.47 \\
{[12.25,18.70]}\end{array}$ & $\begin{array}{c}15.98 \\
{[12.13,19.84]}\end{array}$ & $\begin{array}{c}16.01 \\
{[11.98,20.04]}\end{array}$ & $\begin{array}{c}16.77 \\
{[12.08,21.46]}\end{array}$ & $\begin{array}{c}16.98 \\
12.08,21.87\end{array}$ \\
\hline$E\left[r_{t}^{f}\right]$ & $\begin{array}{c}2.20 \\
{[1.43,2.96]}\end{array}$ & $\begin{array}{c}2.19 \\
{[1.43,2.95]}\end{array}$ & $\begin{array}{c}2.14 \\
{[1.34,2.94]}\end{array}$ & $\begin{array}{c}2.15 \\
{[1.36,2.93]}\end{array}$ & $\begin{array}{c}2.11 \\
{[1.31,2.92]}\end{array}$ & $\begin{array}{c}2.12 \\
1.32,2.92\end{array}$ \\
\hline$\sigma\left[r_{t}^{f}\right]$ & $\begin{array}{c}1.82 \\
{[1.29,2.36]}\end{array}$ & $\begin{array}{c}1.82 \\
{[1.25,2.38]}\end{array}$ & $\begin{array}{c}1.91 \\
{[1.29,2.54]}\end{array}$ & $\begin{array}{c}1.91 \\
{[1.24,2.58]}\end{array}$ & $\begin{array}{c}2.01 \\
{[1.31,2.72]}\end{array}$ & $\begin{array}{c}2.00 \\
1.28,2.73 \\
\end{array}$ \\
\hline \multicolumn{7}{|c|}{ Panel C: Jump intensity in $x_{t}$} \\
\hline & \multicolumn{2}{|c|}{$l_{1}^{x}=0.6 / 12 / \bar{\sigma}$} & \multicolumn{2}{|c|}{$l_{1}^{x}=1 / 12 / \bar{\sigma}$} & \multicolumn{2}{|c|}{$l_{1}^{x}=1.4 / 12 / \bar{\sigma}$} \\
\hline & [1] & {$[2]$} & [1] & {$[2]$} & [1] & {$[2]$} \\
\hline$E\left[r_{t}^{d}-r_{t}^{f}\right]$ & $\begin{array}{c}3.03 \\
{[-0.01,6.08]}\end{array}$ & $\begin{array}{c}3.27 \\
{[0.24,6.31]}\end{array}$ & $\begin{array}{c}3.69 \\
{[0.62,6.75]}\end{array}$ & $\begin{array}{c}4.13 \\
{[0.97,7.29]}\end{array}$ & $\begin{array}{c}4.29 \\
{[1.20,7.39]}\end{array}$ & $\begin{array}{c}4.97 \\
{[1.77,8.17]}\end{array}$ \\
\hline$\sigma\left[r_{t}^{d}-r_{t}^{f}\right]$ & $\begin{array}{c}15.5 \\
{[11.8,19.1]}\end{array}$ & $\begin{array}{c}15.51 \\
{[11.97,19.05]}\end{array}$ & $\begin{array}{c}15.96 \\
{[12.20,19.73]}\end{array}$ & $\begin{array}{c}16.05 \\
{[12.23,19.88]}\end{array}$ & $\begin{array}{c}16.36 \\
{[12.37,20.35]}\end{array}$ & $\begin{array}{c}16.47 \\
{[12.34,20.60]}\end{array}$ \\
\hline$E\left[r_{t}^{f}\right]$ & $\begin{array}{c}2.16 \\
{[1.45,2.87]}\end{array}$ & $\begin{array}{c}2.15 \\
{[1.43,2.87]}\end{array}$ & $\begin{array}{c}2.16 \\
{[1.36,2.96]}\end{array}$ & $\begin{array}{c}2.15 \\
{[1.34,2.96]}\end{array}$ & $\begin{array}{c}2.16 \\
{[1.28,3.04]}\end{array}$ & $\begin{array}{c}2.16 \\
{[1.27,3.05]}\end{array}$ \\
\hline$\sigma\left[r_{t}^{f}\right]$ & $\begin{array}{c}1.77 \\
{[1.21,2.34]}\end{array}$ & $\begin{array}{c}1.77 \\
{[1.17,2.36]}\end{array}$ & $\begin{array}{c}1.97 \\
{[1.35,2.59]}\end{array}$ & $\begin{array}{c}1.96 \\
{[1.32,2.61]}\end{array}$ & $\begin{array}{c}2.15 \\
{[1.43,2.86]}\end{array}$ & $\begin{array}{c}2.13 \\
{[1.44,2.82]} \\
\end{array}$ \\
\hline
\end{tabular}

Notes - The table reports the average and volatilitiy of equity excess return and risk-free rates from Drechsler and Yaron (2011) jump models with alternative calibrations. Column labeled as "[1]" refers to the model in which jump in $x$ has normal distribution, that is, $\xi_{j, t+1}^{x} \sim N\left(0, \sigma_{x}^{2}\right)$. Column labeled as "[2]" refers to the model in which jump in $x$ has demeaned negative exponential distribution, that is, $\xi_{j, t+1}^{x} \sim-\operatorname{Exp}\left(\mu_{x}\right)+\mu_{x}$. Panel A alters the degree of persistence of the expected consumption growth rate, $\rho_{x}$. Panel B alters the jump intensity parameter in $\sigma_{t}$ process $l_{1}^{\sigma}$. Panel C alters the jump intensity parameter in $x_{t}$ process $l_{1}^{x}$. The numbers in squared brackets underneat each statistic are $95 \%$ confidence intervals obtains from 1000 simulations of sample size 100 years. 


\section{REFERENCES}

Anderson, E. 2005. The dynamics of risk-sensitive allocations. Journal of Economic Theory 125(2):93-150.

Arditti, F. 1967. Risk and the required return on equity. Journal of Finance 22(1):19-36.

Azzalini, A. 1985. A class of distributions which includes the normal ones. Scandinavian Journal of Statistics 12:171-178.

Bakshi, G., and F. Chabi-Yo. 2014. New entropy restrictions and the quest for better specified asset pricing models. Ohio State University p. Working Paper.

Bansal, R., and I. Shaliastovich. 2010. Confidence risks and asset prices. American Economic Review 100(2):537-541.

Bansal, R., and I. Shaliastovich. 2011. Learning and asset-price jumps. Review of Financial Studies 24(8):2738-80.

Bansal, R., and I. Shaliastovich. 2013. A long-run risks explanation of predictability puzzles in bond and currency markets. Review of Financial Studies 26(1):1-33.

Bansal, R., and A. Yaron. 2004. Risks for the long run: A potential resolution of asset pricing puzzles. Journal of Finance 59:1481-1509.

Bekaert, G., and E. Engstrom. 2010. Asset return dynamics under bad environment-good environment fundamentals. Columbia University p. Working Paper.

Bekaert, G., E. Engstrom, and A. Ermolov. 2015. Bad environments, good environments: A nongaussian asymmetric volatility model. Journal of Econometrics $\mathrm{p}$. forthcoming.

Bekaert, G., and M. Hoerova. 2014. The VIX, the variance premium and stock market volatility. Journal of Econometrics 183(2):181-192.

Benzoni, L., P. Collin-Dufresne, and R. Goldstein. 2011. Explaining asset pricing puzzles associated with the 1987 market crash. Journal of Financial Economics 101:552-573.

Bollerslev, T., G. Tauchen, and H. Zhou. 2009. Expected stock returns and variance risk premia. Review of Financial Studies 22(11):4463-4492. 
Bollerslev, T., and V. Todorov. 2011. Tails, fears, and risk premia. Journal of Finance 66(6):21652211.

Campbell, J. Y., and J. H. Cochrane. 1999. By force of habit: A consumption based explanation of aggregate stock market behavior. Journal of Political Economy 107:205-251.

Campbell, J. Y., and R. J. Shiller. 1988. Stock prices, earnings, and expected dividends. Journal of Finance 43(3):661-676.

Campbell, S., and F. Diebold. 2009. Stock returns and expected business conditions: Half a century of direct evidence. Journal of Business and Economic Statistics 27:266-278.

Chabi-Yo, F. 2012. Pricing kernels with stochastic skewness and volatility risk. Management Science 58(3):624-640.

Christoffersen, P., and F. X. Diebold. 2006. Financial asset returns, direction of change forecasting, and volatility dynamics. Management Science 52:1273-1287.

Colacito, R. 2008. Six anomalies looking for a model. A consumption based explanation of international finance puzzles. Working Paper, Department of Finance, University of North Carolina, Chapel Hill NC.

Colacito, R., and M. Croce. 2013. International asset pricing with recursive preferences. Journal of Finance 68(6):2651-2686.

Colacito, R., and M. M. Croce. 2011. Risks for the long run and the real exchange rate. Journal of Political Economy 119(1):153-182.

Drechsler, I., and A. Yaron. 2011. What's Vol got to do with it. Review of Financial Studies 24(1):145.

Epstein, L. G., and S. E. Zin. 1989. Substitution, risk aversion, and the temporal behavior of consumption and asset returns: A theoretical framework. Econometrica 57:937-69.

Garcia, J. A., and A. Manzanares. 2007. Reporting biases and survey results evidence from european professional forecasters. European Central Bank Working Paper Series, No. 836:4463-4492.

Ghysels, E. 2014. Conditional skewness with quantile regression models: SoFiE Presidential Address and a Tribute to Hal White. Journal of Financial Econometrics 12:620-644. 
Ghysels, E., A. Plazzi, and R. I. Valkanov. 2011. Conditional skewness of stock market returns in developed and emerging markets and its economic fundamentals. Swiss Finance Institute Research Paper, 11-06.

Hansen, L., and T. J. Sargent. 1995. Discounted linear exponential quadratic gaussian control. IEEE Transactions Automatic Control 40(5):968-971.

Harvey, C. R., and A. Siddique. 2000. Conditional skewness in asset pricing tests. Journal of Finance $55: 1263-1295$.

Havranek, T. 2014. Measuring intertemporal substitution: The importance of method choices and selective reporting. Czech National Bank and Charles University, Working Paper .

Kopecky, K., and R. Suen. 2010. Risk-sensitive real business cycles. Review of Economic Dynamics $13: 701-714$.

Kraus, A., and R. Litzenberger. 1976. Skewness preference and the valuation of risk assets. Journal of Finance 31(4):1085-1100.

Lettau, M., and S. Ludvigson. 2001. Consumption, aggregate wealth, and expected stock returns. Journal of Finance 56(3):815-849.

Lew, R. 1981. An approximation to the cumulative normal distribution with simple coefficients. Applied Statistics 39:299-301.

Rubinstein, M. 1973. The fundamental theorem of parameter-preference security valuation. Journal of Financial and Quantitative Analysis 8(1):61-69.

Segal, G., I. Shaliastovich, and A. Yaron. 2015. Good and bad uncertainty: Macroeconomic and financial markets implications. Journal of Financial Economics p. forthcoming.

Tallarini, T. 2000. Risk-sensitive real business cycles. Journal of Monetary Economics 45:507-532.

Tsai, J., and J. Wachter. 2014. Rare booms and disasters in a multi-sector endowment economy. University of Pennsylvania p. Working Paper.

Wachter, J. A. 2013. Can time-varying risk of rare disasters explain aggregate stock market volatility? Journal of Finance p. forthcoming. 
Welch, I., and A. Goyal. 2008. A comprehensive look at the empirical performance of equity premium prediction. Review of Financial Studies 21(4):1455-1508.

Yang, W. 2011. Long-run risk in durable consumption. Journal of Financial Economics 102:45-61.

Zacks, S. 1981. Parametric statistical inference. Pergamon Press, Oxford . 